\title{
MODELLING REGIME SHIFTS OF CORAL REEFS TO SPONGE REEFS
}

BY

\section{ALBERTO ROVELLINI}

A thesis submitted to the Victoria University of Wellington

In fulfilment of the requirements for the degree of

Doctor of Philosophy

\section{VICTORIA UNIVERSITY OF WELLINGTON \\ Te Whare Wānanga o te Ūpoko o te Ika a Māui}

2020

TE WHARE WĀNANGA O TE ŪPOKO O TE IKA A MĀUI

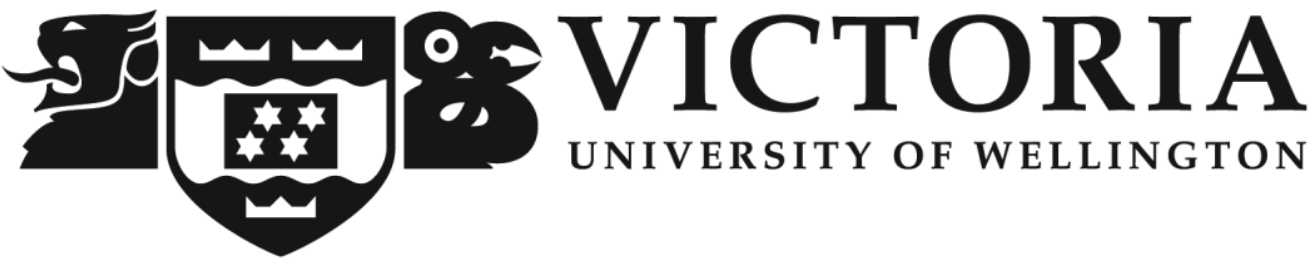


This thesis was conducted under the supervision of:

\author{
Prof James J. Bell
}

(Primary supervisor, Victoria University of Wellington)

$\&$

Dr Matthew R. Dunn

(Co-supervisor, National Institute of Water and Atmospheric Research - NIWA New Zealand)

$\&$

Dr Elizabeth A. Fulton

(External supervisor, Commonwealth Scientific and Industrial Research Organization - CSIRO - Australia) 


\section{Abstract}

Coral reef ecosystems have been degrading globally for decades due to global climate change and anthropogenic pressure, and corals are expected to continue declining in the future. Some coral reef sponges, although also vulnerable to warmer seawater, are more tolerant than corals to the combined effects of projected ocean warming and acidification. As a result, sponges have been proposed as potential winners on future coral reefs. Shifts towards sponge dominance on coral reefs may result into altered reef trophodynamics. However, there is limited information on how ecological processes on coral reefs will be affected by an increase in sponge dominance. Therefore, the potential ecological functioning of sponge-dominated reefs is unclear. To understand how reef ecosystems could function if sponges will become dominant, it is important to clarify: how perturbations that have a negative effect on corals propagate through the reef ecosystem; how sponge assemblages can develop over time; and how increased sponge dominance will affect other coral reef organisms. Focusing on IndoPacific coral reefs, this thesis investigates the temporal dynamics and the ecological processes that characterise reefs where sponges form abundant and highly diverse assemblages. Aims of this thesis were: 1) to explore the ecosystem-level effects of climate change-induced shifts towards sponge dominance on coral reef ecosystems; 2) to quantify the temporal variability of sponge abundance and biodiversity on coral reefs; 3) to understand how benthic sessile organisms benefit from free substrate that becomes available when corals die; 4) and to determine whether sponges can provide structural complexity similar to that of corals.

In my first data chapter, I use qualitative mathematical modelling to identify the key ecological processes that determine the functioning of a sponge-dominated reef. With a set of qualitative models, I obtain qualitative predictions of the ecosystem-level effects of increased temperature, acidity, and turbidity on a coral reef ecosystem, and of a shift towards sponge dominance. Model predictions showed that simulated environmental change caused coral decline, subsequent loss of reef structural complexity, and increased sponge and macroalgal abundance, while enhancing secondary productivity and detrital pathways. By analysing the uncertainty around model predictions, I highlight the need to elucidate some aspects of sponge ecology to better understand how sponge-dominated reef may function. These processes include temporal changes in sponge assemblages, competitive interaction between sponges and algae, and the contribution of sponges to reef structural complexity. I address these aspects in the following chapters. 
In my second data chapter, I characterise the temporal variability of an abundant and diverse sponge assemblage on an Indonesian coral reef (Hoga Island, Wakatobi Marine Park, SE Sulawesi), studying temporal changes in sponge abundance and biodiversity over 13 years (2005-2017). Mean sponge abundance showed large temporal variability at this reef over the study period. In total, 141 sponge taxa were recorded at this reef over the study period, and species composition of the assemblage showed strong temporal patterns that were determined by the population dynamics of a few abundant taxa (e.g. Protosuberites sp., Sycon sp., and Pericharax sp., on average $\sim 25 \%, 20 \%$ and $5 \%$ of total sponge abundance, respectively). In this chapter, I highlight that Indo-Pacific sponge assemblages can develop rapidly, undergoing large temporal changes that are driven by species-specific population variability.

In my third data chapter, I describe the temporal dynamics of all benthic organisms on the Hoga reef over the period 2006-2017, and investigate the effects of free space on benthic cover at subsequent sampling events. In contrast with other reefs in the Wakatobi where corals have declined, corals were dominant and stable over time at this site, and no other benthic group increased in abundance over time. However, percentage cover of turf algae and sponges showed larger interannual variability than corals and crustose coralline algae (CCA). Bare substrate was a good predictor of turf percentage cover in the following year across sites. Moreover, percentage cover of algal groups combined with bare space was a good predictor of CCA percentage cover the following year, and of sponge cover at one site. In this chapter, I show that turf algae in the Indo-Pacific rapidly colonise newly available substrate, and that other benthic groups like CCA and sponges may overgrow substrates already fouled by turf.

In my fourth data chapter, I use 3D photogrammetry to compare the structural complexity of coral-dominated and sponge-dominated reef quadrats on Hoga Island, and to identify which coral and sponge growth forms contribute to reef structure. Computer-generated 3D models were used to measure reef rugosity ( $R$, denoting complexity of reef contours), vector dispersion $\left(1 / k\right.$, denoting heterogeneity of the angles between reef surfaces) and fractal dimension $\left(D_{1-5}\right.$, denoting complexity of refuge holes at five different spatial scales between $120-1 \mathrm{~cm}$ ). Structurally complex coral-dominated and sponge-dominated quadrats were similar in terms of coarse scale structural complexity $(R$ and $1 / k)$. However, complexity at the scale of small refuge holes $\left(D_{5}\right)$ was higher for coral-dominated quadrats, indicating that corals provide smaller refugia compared to sponges, mainly due to branching corals. Branching and massive corals determined the overall 3D structure of coral-dominated quadrats, and barrel sponges of spongedominated quadrats. In this chapter I show that, while sponge reefs can be three-dimensionally 
complex if upright sponge growth forms are abundant, sponges do not provide small-scale structural complexity $(5-1 \mathrm{~cm}$ refuge holes) comparably to corals.

In my fifth data chapter, I present how I captured sponges and their ecological roles in the ecosystem model Atlantis, by drawing on the previous data chapters and on the existing literature. I then present my contribution to the development of an Atlantis application for the Australian Great Barrier Reef (GBR), focusing on how sponges were parameterised in this model. Important sponge functional roles were captured in Atlantis either by developing novel components of the Atlantis code, or by modifying existing code specific to other benthic organisms (like corals), in such a way that it would be applicable to sponges. The spongespecific ecological processes added to Atlantis in the scope of this chapter include: competition of sponges for free substrate with other benthic organisms; sponge-provided structural complexity; sponge-mediated bioerosion of carbonates; and Silicon-limitation to sponge growth. This sponge framework was tested with Atlantis GBR. I captured sponges in Atlantis GBR as three groups (heterotrophic, phototrophic, and bioeroding), and I also added to the model one group of spongivorous fishes. I participated to the development and parametrisation of the coral reef benthos of the model, with a focus on sponges. I present the development and calibration (ongoing) of Atlantis GBR.

In summary, in this thesis I found that sponge-dominated reefs could be highly dynamic ecosystems, with different structural properties compared to coral reefs. I discuss how reef trophodynamics are likely to be altered as a result of increased sponge dominance, with potential increases in detrital carbon pathways that will depend on sponge population dynamics. I also highlight that some aspects of sponge ecology still need to be elucidated to better understand how shifts towards sponge dominance may happen, and how the resulting reef states will function. These aspects include the role of sponges as net producers of detritus, the competitive interactions between sponges and algae, and the use of sponge-provided structural complexity by reef fauna. Understanding how sponge-dominated reefs may originate and how they will function will be crucial to maintain the ecological, economic, and social value of coral reefs in the near future. 
A Silvia e Gianluca. 


\section{Acknowledgements}

I am grateful to the amazing group of scientists I had the privilege to work with in the past three years, and that have made this thesis possible. Firstly, I wish to thank my primary supervisor at Victoria University of Wellington, Prof James Bell, for his excellent academic mentoring and constant support, for always providing invaluable feedback to my many manuscript drafts, and, most importantly, for the many opportunities he has offered me that have made my PhD years so very rich and stimulating. I also thank my secondary supervisor in Wellington, Dr Matt Dunn (NIWA), for always being approachable and supportive, for always giving me helpful and positive advice throughout my $\mathrm{PhD}$, and for his excellent feedback on my manuscripts (and for the coffees). I am also thankful to my external supervisor, Dr Beth Fulton (CSIRO), for welcoming me to her inclusive and stimulating research group during my time in Hobart, for her support with model development and calibration, for motivating me to participate in discussions about Atlantis, and for her valuable input to my manuscripts.

This research was possible thanks to the Royal Society of New Zealand's Marsden fund that provided my scholarship and covered my university fees. I also thank Victoria University of Wellington for providing a Submission Scholarship, as well as resources and support for my research and training. Funding from Victoria University of Wellington, Operation Wallacea, and the PADI Foundation supported previous work in Indonesia, which made Chapter 3 and Chapter 4 possible. I am grateful to Operation Wallacea for providing accommodation and support on Hoga Island (Indonesia) in 2017, with special thanks to Pippa Mansell, Rowan Watt-Pringle, and the local Indonesian team for facilitating my fieldwork. I am also thankful to CSIRO for having me as visiting PhD student in Hobart (Australia) in 2016 and 2018.

Special thanks go to: Dr Nicole Webster and the Australian Institute of Marine Science (AIMS), for providing access to the AIMS Long-term Monitoring Program data used in the Atlantis model for the Great Barrier reef; Daniel "Snout" McNaughtan (VUW), for making me improve as a diver, and for always facilitating diving operations and access to diving equipment; Dr Lisa Woods (VUW), for her helpful advice on statistical analyses for Chapter 4; Charli Mortimer (VUW), for sharing the load of fieldwork for Chapter 5; Rowan WattPringle (Operation Wallacea), for his help in the field on Hoga Island; Dr Jade Berman, for her contribution to sponge identification for Chapter 3; Bec Gorton (CSIRO), for answering my questions about the Atlantis code, and for being helpful with software troubleshooting; Dr 
Shane Richards (CSIRO), for useful input on earlier versions of Chapter 3; and Dr Heidi Pethybridge (CSIRO), for useful discussion about the Atlantis model.

I am thankful for the many friends I was fortunate enough to meet over these years. Each one of you has taught me something, and in different ways you all have made my time here unforgettable. Thanks to all members of my lab group, AKA "Sponge club", for being so welcoming when I first turned up, and for all the good times ever after. I also thank the VUCEL crew, for making sure I was never too focused for too long. A special thank you goes to Mat and Liv, my very first friends in New Zealand.

I am forever grateful for the unconditional support of my parents, Silvia and Gianluca. You have always encouraged me to go and do what I really liked, no matter the distance, no matter the goodbyes. None of this would have been possible without your love, your wisdom, and your faith in me. Finally, Meg, I have been incredibly lucky to find you along this path. Thank you for making these years exciting and wonderful, for all the adventures, for being such a solid support system, and for always helping me remember what really is important. 


\section{Chapter contributions and Publications}

\section{Chapter 2:}

Study design: Alberto Rovellini, Prof James J. Bell (Victoria University of Wellington VUW - New Zealand), and Dr Nicole S. Webster (Australian Institute of Marine Science AIMS - Australia). In addition, Dr Matthew R. Dunn (National Institute of Water and Atmospheric Research - NIWA - New Zealand), Dr Elizabeth A. Fulton (Commonwealth Scientific and Industrial Research Organization - CSIRO - Australia), Prof Simon K. Davy (VUW), Dr Michael W. Taylor (University of Auckland), Dr Holly M. Bennett (VUW), Nora M. Kandler (VUW), and Dr Heidi M. Luter (AIMS) contributed to discussion of the study design.

Data collection / modelling: AR.

Data analyses: AR.

Chapter writing and editing: $\mathrm{AR}, \mathrm{JJB}, \mathrm{MRD}, \mathrm{EAF}$.

Publication: Bell, J. J., Rovellini, A., Davy, S. K., Taylor, M. W., Fulton, E. A., Dunn, M. R., Bennett, H. M., Kandler, N. M., Luter, H. M., and Webster, N. S. (2018). Climate change alterations to ecosystem dominance: how might sponge-dominated reefs function? Ecology, 99(9):1920-1931. https://doi.org/10.1002/ecy.2446

Note: This chapter as it is reported in this thesis does not represent a copy of the paper that was produced from this work (Bell et al. 2018a). The paper version of this work was the collaborative effort between several working groups. AR contributed to the Bell et al. (2018a) by carrying out the modelling work (model development, model runs, and analysis of the results). The chapter version, however, was developed in full by AR, who wrote the piece as a traditionally-structured study (Introduction-Methods-Results-Discussion), rather than an opinion piece like Bell et al. (2018a). In this, AR was supervised by his academic advisors JJB, MRD, EAF.

\section{Chapter 3:}

Study design: AR with the guidance of JJB, MRD, and EAF.

Data collection / sponge identification: JJB and Jade Berman (VUW). 
Data analyses: AR.

Chapter writing and editing: AR, JJB, MRD, EAN. In addition, NSW, Prof David J. Smith (University of Essex, UK), Prof Jamaluddin Jompa and Prof Abdul Haris (Universitas Hasanuddin, Indonesia), and JB proofread the manuscript.

Publication: Rovellini A., Dunn, M. R., Fulton, E. A., Webster, N. S., Smith, D. J., Jompa, J., Haris, A., Berman, J., and Bell, J. J. (2019) Decadal variability in sponge abundance and biodiversity on an Indo-Pacific coral reef. Marine Ecology Progress Series, 620:63-76. https://doi.org/10.3354/meps12968

\section{Chapter 4:}

Study design: AR with the guidance of JJB, MRD, and EAF.

Data collection: JJB performed in situ photographic surveys. AR analysed the photographs.

Data analyses: AR, with assistance from Dr Lisa Woods (VUW).

Chapter writing and editing: $\mathrm{AR}, \mathrm{JJB}, \mathrm{MRD}, \mathrm{EAF}$.

Publication: Rovellini A., Dunn, M. R., Fulton, E. A., Woods, L., J., Jompa, J., Haris, A., and Bell, J. J. Decadal stability and interannual variability of benthic organisms on an Indo-Pacific coral reef. In prep.

\section{Chapter 5:}

Study design: AR with the guidance of JJB, MRD, and EAF, and with the participation of Charlotte Mortimer (VUW).

Data collection: AR and CM.

Data analyses: AR.

Chapter writing and editing: $\mathrm{AR}, \mathrm{JJB}, \mathrm{MRD}, \mathrm{EAF}$.

Publication: Rovellini A., Mortimer, C., Dunn, M. R., Fulton, E. A., J., Jompa, J., Haris, A., and Bell, J. J. Reduced structural complexity on sponge-dominated reefs limits fish habitat availability at small spatial scales. In prep. 


\section{Chapter 6:}

Study design: AR with the guidance of JJB, MRD, and EAF.

Development and coding of new sponge components into Atlantis: AR, with assistance from EAF for coding.

Model testing and parametrisation: AR.

Great Barrier Reef model calibration: AR and EAF.

Chapter writing and editing: AR, JJB, MRD, EAF.

Publication: Rovellini A., Fulton, E. A., Dunn, M. R., and Bell, J. J. Modelling sponges in the Atlantis ecosystem model. In prep. 


\section{Table of Contents}

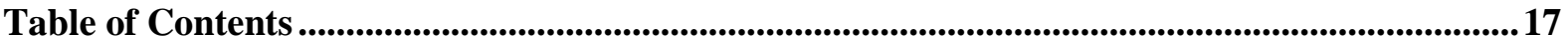

List of Figures............................................................................................................................... 21

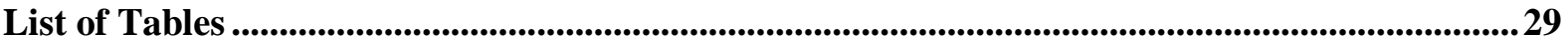

Chapter 1. General Introduction .................................................................................................31

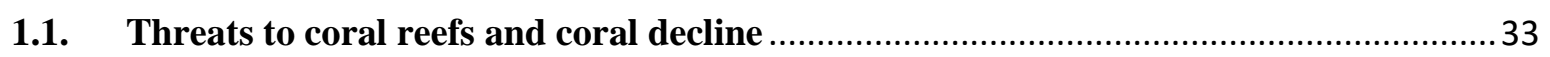

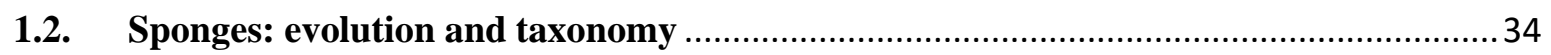

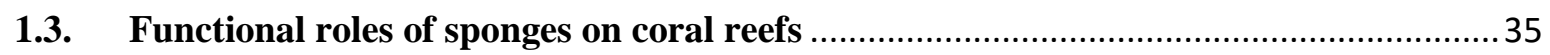

1.3.1. Filtration, pumping, and biogeochemical cycling ........................................ 35

1.3.2. Reef building and degradation............................................................. 36

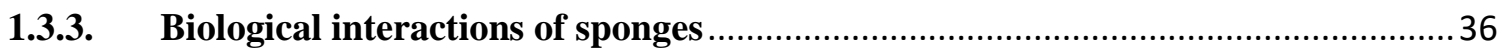

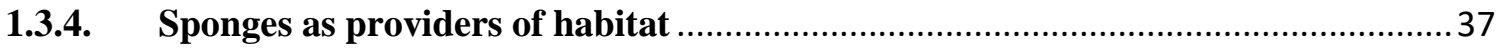

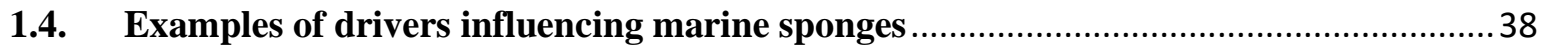

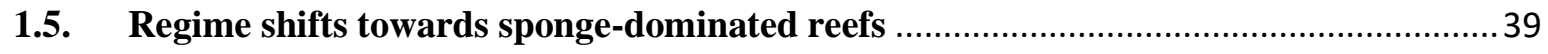

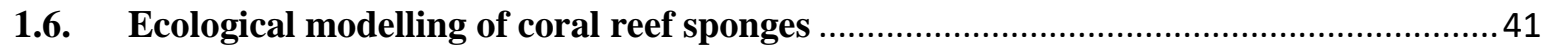

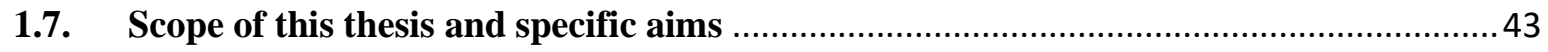

Chapter 2. Qualitative modelling of alternative reef states.................................................45

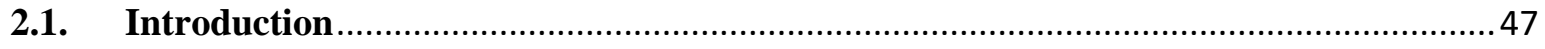

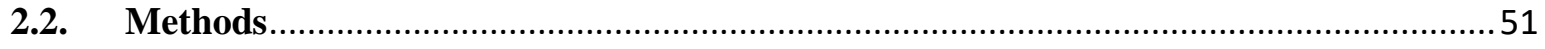

2.2.1. Qualitative modelling of natural systems: an overview ................................. 51

2.2.2. A qualitative model of a coral reef: model assumptions.................................53

2.2.3. Model application: cascading effects of shifts in benthic dominance ...................56

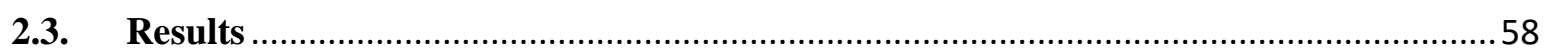

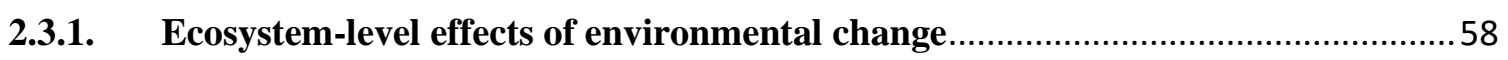

2.3.2. Ecosystem-level effects of changes in benthic dominance ...................................59

2.3.3. Roles of structural complexity, sponge loop, and competition in regime shifts.....60

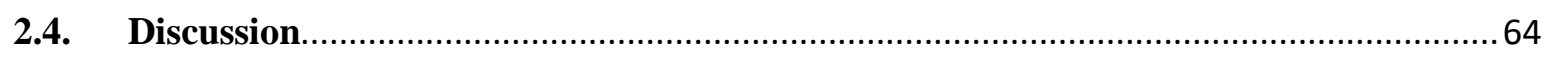

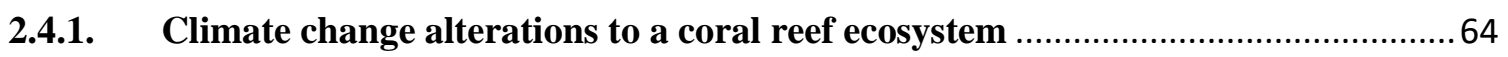

2.4.2. Ecosystem-level effects of changes in benthic dominance ..................................66

2.4.3. Key ecological processes in shifts towards sponge dominance: alternative models 67

2.4.4. Emerging key questions and focus of future chapters 69

Chapter 3. Temporal variability of sponge abundance and biodiversity of an Indonesian sponge assemblage.

3.1. Introduction 


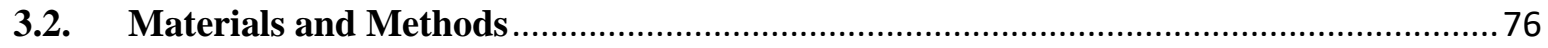

3.2.1. Site description and sampling protocol .............................................................. 76

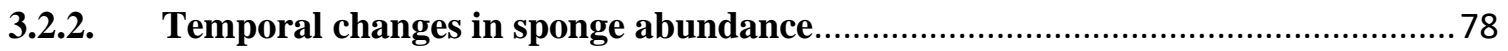

3.2.3. Effects of sea-surface temperature on sponge abundance and growth .................. 78

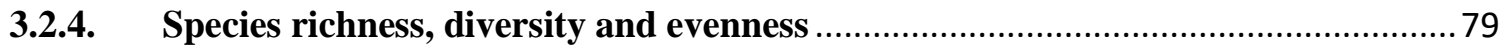

3.2.5. Spatio-temporal changes in species composition................................................. 80

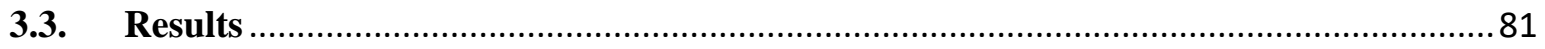

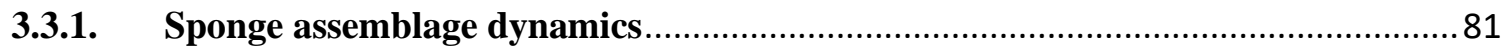

3.3.2. Effects of water temperature on sponge abundance and growth ........................... 83

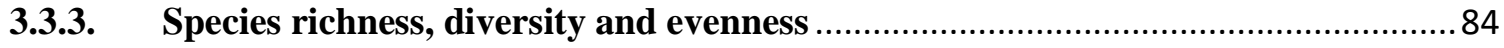

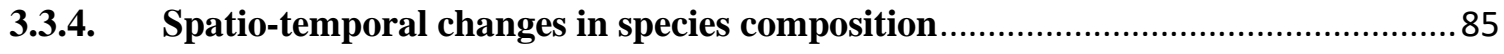

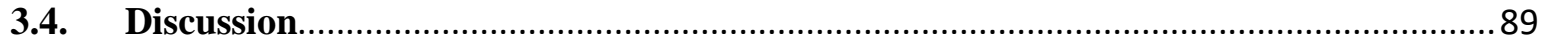

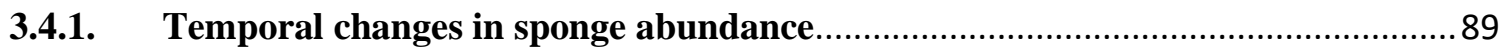

3.4.2. Temporal changes in taxonomic composition and biodiversity ............................. 90

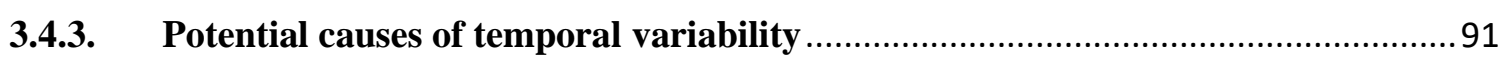

3.4.4. Implications of assemblage variability for coral reef monitoring.......................... 93

Chapter 4. Decadal stability and interannual variability of benthic organisms on an Indo-Pacific

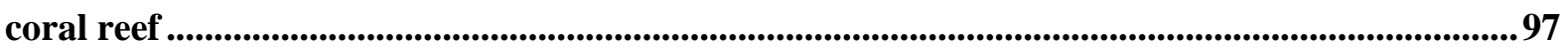

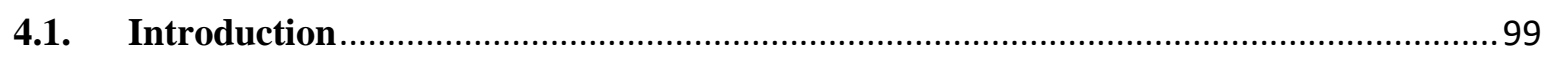

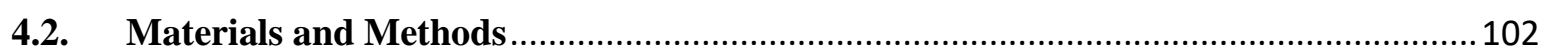

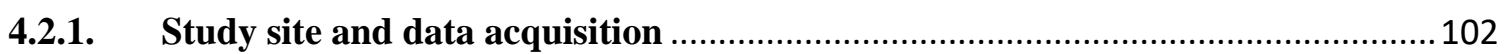

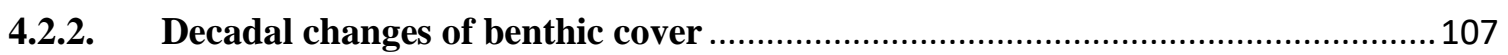

4.2.3. Spatial and temporal variation of community structure ....................................... 108

4.2.4. Interannual changes of benthic cover of live benthic taxonomic groups .............108

4.2.5. Lagged effects of free space on benthic cover.................................................... 109

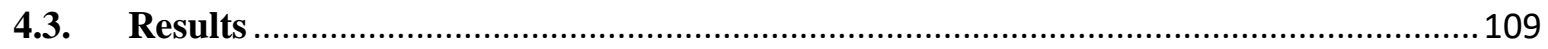

4.3.1. Temporal changes in percentage cover .............................................................. 109

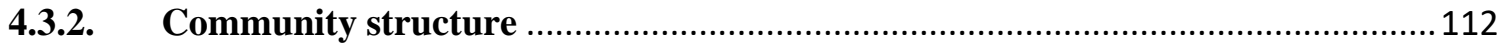

4.3.3. Interannual changes of benthic cover of taxonomic groups...............................113

4.3.4. Lagged effects of free space on benthic cover..................................................... 114

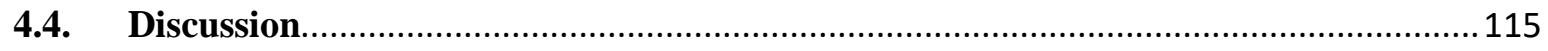

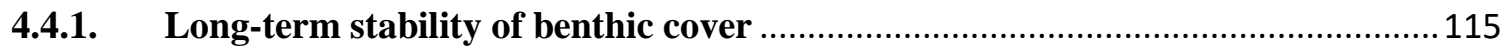

4.4.2. Spatial and temporal variation in community structure.....................................117

4.4.3. Interannual changes in benthic cover ................................................................ 117

4.4.4. Comparing temporal changes in sponge abundance and sponge percentage cover 118 


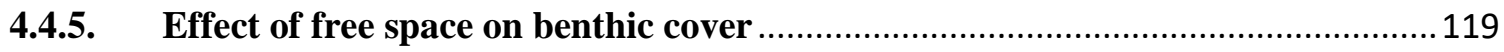

4.4.6. Implications for Indo-Pacific reef dynamics..................................................... 122

Chapter 5. Reduced small-scale structural complexity on sponge-dominated reefs.....................125

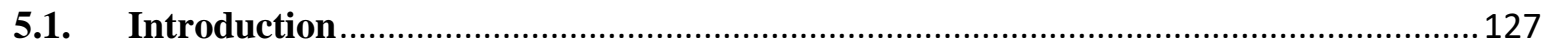

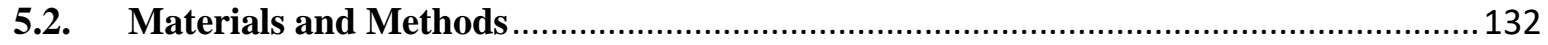

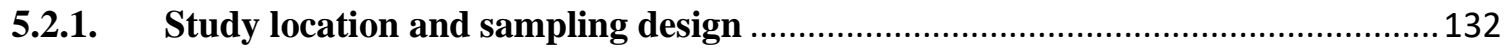

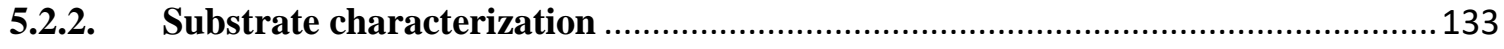

5.2.3. 3D photogrammetry of the reef and complexity metrics …….............................. 134

5.2.4. Structural properties of coral and sponge quadrats ........................................... 137

5.2.5. Contribution of growth forms to overall 3D structure ....................................... 138

5.2.6. Contribution of growth form to specific complexity metrics ................................ 138

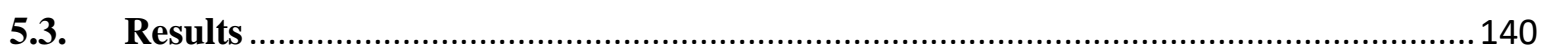

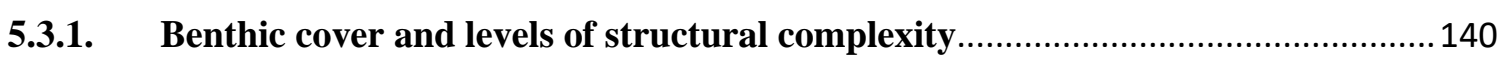

5.3.2. Structural properties of coral and sponge quadrats ............................................ 142

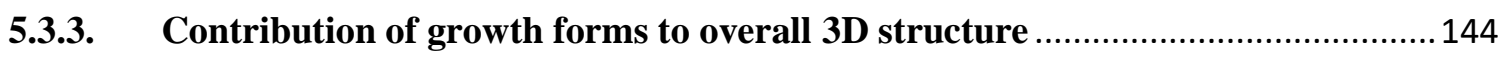

5.3.4. Contribution of growth forms to complexity metrics .......................................... 145

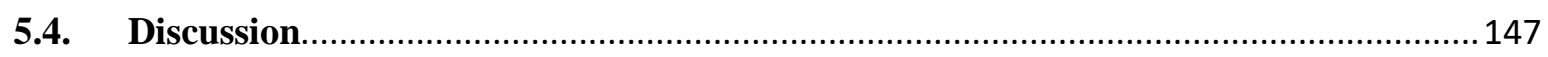

5.4.1. Structural properties of coral and sponge quadrats ........................................... 147

5.4.2. Contribution of morphologies to overall 3D structure and to complexity metrics 150

Chapter 6. A framework to model sponges in the ecosystem model Atlantis.................................155

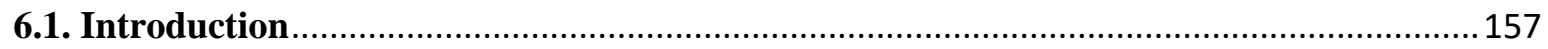

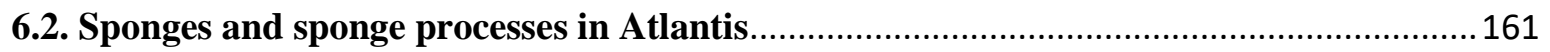

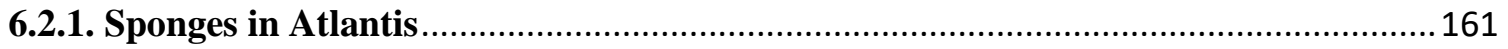

6.2.2. Benthic competition: growth limitation and smothering ........................................ 162

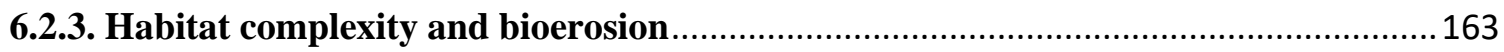

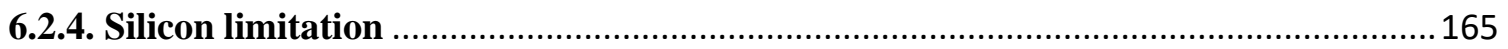

6.3. Other processes: a sub-model for seagrass cover-to-biomass conversions ...................... 165

6.4. Model application: Atlantis Great Barrier Reef ........................................................... 167

6.4.1. Cover and biomass of benthic organisms in Atlantis GBR …................................. 167

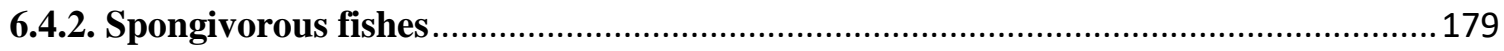

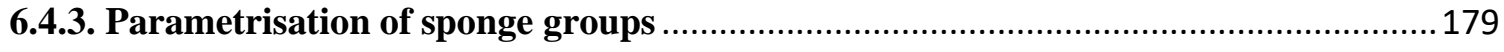

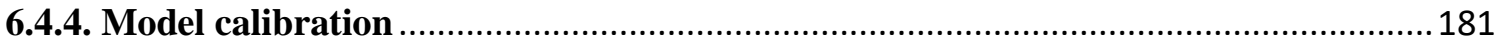

Chapter 7. General Discussion........................................................................................................................183

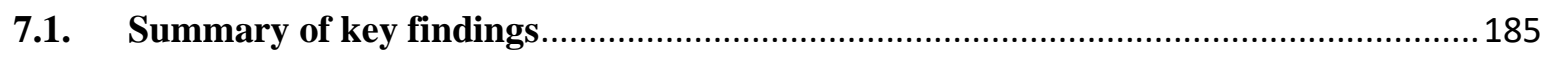

7.2. Sponge temporal variability: limitations to increases in sponge abundance ...............186 


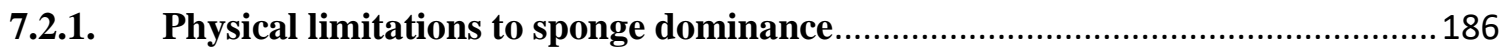

7.2.2. Competitive limitations to sponge dominance .................................................... 188

7.2.3. Bottom-up biological limitations to sponge-dominance....................................... 190

7.2.4. Top-down biological limitations to sponge dominance......................................... 191

7.3. Sponge reefs: what could they look like? ................................................................... 191

7.3.1. Ecological functioning of sponge reefs ……....................................................... 191

7.3.2. Sponges to not functionally replace corals......................................................... 193

7.4. Modelling regime shifts of coral reefs to sponge reefs .............................................. 193

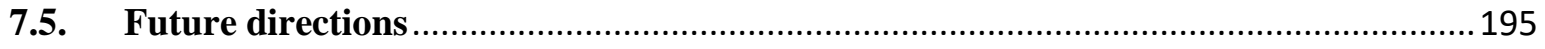

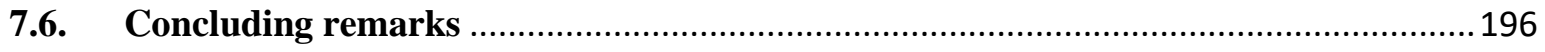

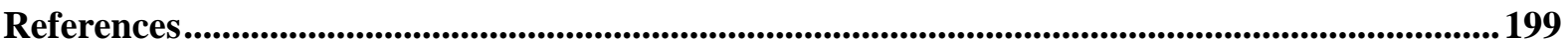

Appendix 1. Overview of the qualitative modelling approach.................................................249

Appendix 2. Temporal variability of sponge abundance and biodiversity of an Indonesian

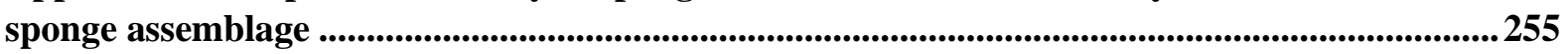

Appendix 3. Decadal stability and interannual variability of benthic organisms on an Indo-

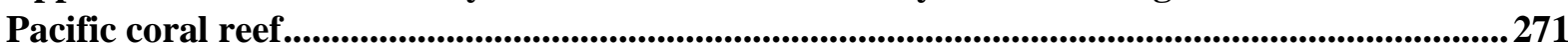




\section{List of Figures}

Figure 2.1. Signed digraph of a predator (2) feeding on a prey item (1). Both variables are selfdampened, meaning they have a negative self-effect (see text)

Figure 2.2. Qualitative models of a coral reef. (a) Complete - QM1; (b) simplified model QM2; (c) structural complexity model - QM3; (d) sponge loop model - QM4; (e) macroalgal competitive superiority model - QM5. In order from top to bottom: piscivorous fishes (PF), spongivorous fishes (SF), grazers (GR), structural complexity (SCX), turbidity (TU), detritus (DET), macroalgae (MA), acidity (A), free space (FS), dissolved organic carbon (DOC), sponges (SP), hard corals (HC), temperature (T), picoplankton (PIC), zooplankton (ZP), phytoplankton (PHY). The ends of the arrows indicate the effect of the source variable on the target variable, with $(\rightarrow)$ denoting a positive effect, $(-\circ)$ a negative effect, and $(-)$ no effect.

Figure 2.3. Effects of a simultaneous increase in temperature, acidity, and turbidity as potential outcomes of climate change and human impact on coral reefs, with associated responses. In order from top to bottom: piscivorous fishes (PF), spongivorous fishes (SF), grazers (GR), structural complexity (SCX), turbidity (TU), detritus (DET), macroalgae (MA), acidity (A), free space (FS), dissolved organic carbon (DOC), sponges (SP), hard corals (HC), temperature (T), picoplankton (PIC), zooplankton (ZP), phytoplankton (PHY). The ends of the arrows indicate the effect of the source variable on the target variable, with $(\rightarrow)$ denoting a positive effect, (-०) a negative effect, and (-) no effect. Free space was incorporated in the model as a state variable in order to explicitly represent passive occupation of the substrate, alongside specific interactions between benthic groups. However, increases or decreases in free space were not reported as model results, because fluctuations in free space would be difficult to interpret in the context of three benthic groups competing for it. Modified from Bell et al. (2018a), Fig. 1

Figure 2.4. Effects of changes in benthic dominance predicted by QM2. (a) Perturbation provides a negative input on corals. (b) Perturbation provides a positive input on sponges. In order from top to bottom: piscivorous fish (PF), spongivorous fish (SF), grazers (GR), macroalgae (MA), free space (FS), sponges (SP), hard corals (HC), picoplankton (PIC), zooplankton (ZP), phytoplankton (PHY). The ends of the arrows indicate the effect of the 
source variable on the target variable, with $(\rightarrow)$ denoting a positive effect, $(-\circ)$ a negative effect, and (-) no effect. Modified from Bell et al. (2018a), Fig. 2.

Figure 2.5 Effects of changes in benthic dominance predicted by QM3 (a-b) and QM4 (c-d). $(a, c)$ Response of each system to negative press perturbation acting on corals; (b, d) response of each system to positive press perturbation acting on sponges. In order from top to bottom: piscivorous fish (PF), spongivorous fish (SF), grazers (GR), structural complexity (SCX), macroalgae (MA), free space (FS), sponges (SP), hard corals (HC), picoplankton (PIC), zooplankton (ZP), phytoplankton (PHY), detritus (DET), dissolved organic carbon (DOC). The ends of the arrows indicate the effect of the source variable on the target variable, with $(\rightarrow)$ denoting a positive effect, $(-\circ)$ a negative effect, and (-) no effect. Modified from Bell et al. (2018a), Fig. 3

Figure 2.6. Effects of changes in benthic dominance predicted by QM5. (a) Perturbation provides a negative input on corals. (b) Perturbation provides a positive input on algae. In order from top to bottom: piscivorous fish (PF), spongivorous fish (SF), grazers (GR), macroalgae (MA), free space (FS), sponges (SP), hard corals (HC), picoplankton (PIC), zooplankton (ZP), phytoplankton (PHY). The ends of the arrows indicate the effect of the source variable on the target variable, with $(\rightarrow)$ a positive effect, $(-\circ)$ a negative effect, and (-) no effect. Modified from Bell et al. (2018a), Fig. 4.

Figure 3.1. Map of Hoga Island, the Wakatobi National Marine Park, and Sulawesi. The star indicates the location of the study site. Map data: (C) OpenStreetMap contributors, available from https://www.openstreetmap.org. Map created in QGIS (QGIS Development Team 2018).

Figure 3.2. Mean total sponge abundance from 2005-2017 at the three sites (letters A-C) at Buoy 3 (Hoga Island, Wakatobi National Marine Park, Indonesia). .81

Figure 3.3. Decomposed sea surface temperature (SST) time series data. Random, seasonal, and trend component extracted with moving average decomposition. .83

Figure 3.4. Decomposed sea surface temperature anomalies (SSTANOM) time series data. Random, seasonal, and trend component extracted with moving average decomposition...... 84 
Figure 3.5. Effect plots showing the best linear mixed models (LMMs) fits for the biodiversity metrics (species richness S, Shannon's diversity H', and Simpson's evenness E1/D) modelled as function of sponge counts and site. Blue lines: best LMM fit; black dashed lines: 95\% confidence intervals of the model parameters; grey dots: observed data. Different shapes of the dots represent different quadrats within each site.

Figure 3.6. Spatial and temporal variation of sponge assemblage composition. A: Non-metric Multi-dimensional Scaling (nMDS) plot for the sponge assemblages at the three sites between 2005 and 2017. Each sample corresponds to a quadrat. Symbols indicate the year, colours the site. B: Canonical Analysis of Principal coordinates (CAP) plot. Years and sites represent the centroid for each set of samples. For visualisation purposes, only the taxonomically identified taxa that varied the most between sites and years are plotted (but all taxa were included in the analysis). See Table 3.2 for species abbreviations. .88

Figure 4.1. Map of Hoga Island, with insets showing the location of the Wakatobi National Marine Park and Sulawesi. The star indicates the location of the study site off Hoga Island.

Figure 4.2. Mean percentage cover of benthic organisms at the Buoy 3 reef. Different columns $(\mathrm{A}, \mathrm{B}, \mathrm{C})$ indicate the three sites. Vertical bars represent the standard error of the mean. Sites $\mathrm{A}$ and $\mathrm{B}$ are placed on a vertical wall, whereas Site $\mathrm{C}$ is a steep incline (approx. $80^{\circ}$ incline). Note the different scales for different benthic categories. 110

Figure 4.3. Ternary plots showing the relative proportions of the three main benthic competitors: hard corals, sponges and algae. Dots indicate single photoquadrats. The closer the dot is to a corner of the ternary plot, the more dominant the group in that corner is. Letters A, $\mathrm{B}$, and $\mathrm{C}$ indicate the three sites.

Figure 4.4. Spatial and temporal variation of community structure. A: Non-metric Multidimensional Scaling (nMDS) plot for the benthic community at the three sites in surveyed years between 2006 and 2017. Each dot corresponds to a quadrat. Symbols indicate the year, colours the site. B: Canonical Analysis of Principal coordinates (CAP) plot. Years and sites represent the centroid for each set of samples. HC: hard corals; SP: sponges; AL: algae; CA: CCAs; OT: other invertebrate; FS: free space; AB: abiotic (sand and silt). Category "Undetermined" not shown in CAP. 
Figure 4.5. Box-plots showing the interannual variability of percentage cover of the benthic taxonomic groups, expressed as $\log (\varphi)=\log \left(\mathrm{C}_{1} / \mathrm{C}_{0}\right)$, where $\mathrm{C} 1$ is the cover of group $\mathrm{X}$ at time $\mathrm{y}+1$ and $\mathrm{C}_{0}$ is the cover of group $\mathrm{X}$ in year $\mathrm{y}$. Dashed horizontal line represents $\log \left(\mathrm{C}_{1} / \mathrm{C}_{0}\right)=0$, that is $\mathrm{C}_{1} / \mathrm{C}_{0}=1$, equal to no change between consecutive years. A large spread from the zeroline indicates high interannual variability for a group.

Figure 5.1. Study location on Hoga Island, Wakatobi Marine Park, SE Sulawesi (Indonesia). Red dots indicate the two reefs where the sampling occurred.

Figure 5.2. Most common coral (A) and sponge (B) growth forms found on Hoga Island. Corals: 1: branching; 2: foliose; 3: table; 4: massive; 5: submassive; 6: free-living (mushroom); 7: encrusting. Sponges: 8: branching; 9: tubular; 10: repent; 11: foliose (fan-shaped); 12: digitate; 13: barrel; 14: massive; 15: cushion; 16: globular; 17: encrusting. 135

Figure 5.3. Examples of 3D models of $4 \mathrm{~m}^{2}$ quadrats, with table reporting the respective values for the 7 metrics of structural complexity. (a) High-complexity coral-dominated quadrat (Q06), dominated by branching corals and characterised by high heterogeneity of the surfaces $(1 / \mathrm{k})$ and intermediate- to small refuge space complexity ( $\left.\mathrm{D}_{3-5}\right)$; (b) high-complexity spongedominated quadrat (Q02), with tubular sponges, characterised by high rugosity (low R) and large refuge space complexity $\left(\mathrm{D}_{1}\right)$; (c) low-complexity coral-dominated quadrat (Q24), and (d) low-complexity sponge-dominated quadrat (Q14), both characterised by encrusting growth forms and comparatively low values for most metrics. 136

Figure 5.4. Percentage cover of benthic taxonomic groups and abiotic substrates in the $324 \mathrm{~m}^{2}$ quadrats.

Figure 5.5. Hierarchical clustering based on the matrix of Euclidean distances calculated on the multivariate matrix of the 7 structural complexity metrics. A: coral-dominated quadrats. B: sponge-dominated quadrats. Blue quadrats are high-complexity, red quadrats are low complexity. Coral-dominated and sponge-dominated quadrats are denoted by the letters $\mathrm{C}$ and $\mathrm{S}$, respectively. High-complexity and low-complexity quadrats are denoted by the letters $\mathrm{H}$ and $\mathrm{L}$, respectively. 141

Figure 5.6. A: percentage cover of coral morphologies in coral-dominated quadrats; B: percentage cover of sponge morphologies in sponge-dominated quadrats. 142 
Figure 5.7. Boxplots of the model-derived habitat complexity metrics for coral (red) and sponge (blue) quadrats, at low and high levels of structural complexity. Note the different scales on the y axis for different metrics. Asterisks indicate significant differences reported by the Generalised Linear Models, either between coral and sponge quadrats (asterisk in between boxes) or between levels of complexity for the group indicated by the letter (asterisk in the centre of the plot, $\mathrm{c}=$ corals, $\mathrm{s}=$ sponges). $\mathrm{R}=$ rugosity (note the inverse scale), $1 / \mathrm{k}=$ vector dispersion, $D_{1-5}=$ fractal dimension. Numbers in the first panel represent sample size $(N) .143$

Figure 5.8. Redundancy Analysis plots that show which growth forms correlate with high- and low-complexity quadrats, and how quadrats are distributed in the multivariate space defined by the complexity metrics. A: coral-dominated quadrats and coral morphologies. B: spongedominated quadrats and sponge morphologies. $\mathrm{R}=$ rugosity (note the inverse scale), $1 / \mathrm{k}=$ vector dispersion, $\mathrm{D}_{1-5}=$ fractal dimension, $\mathrm{BRA}=$ branching, $\mathrm{TAB}=$ table, $\mathrm{FOL}=$ foliose MAS = massive, $\mathrm{SUB}=$ sub-massive, FRL = free-living, $\mathrm{ENC}=$ encrusting, $\mathrm{TUB}=$ tubular, $\mathrm{REP}=$ repent, $\mathrm{DIG}=$ digitate, $\mathrm{BAR}=$ barrel, $\mathrm{CUS}=$ cushion, $\mathrm{GLO}=$ globular. 144

Figure 5.9. Mean values of the LASSO regression coefficients of percentage cover of the growth forms as predictors of the 7 complexity metrics, estimated at $\lambda_{\min }$ in 100 random iterations of the cross-validation. A: coral-dominated quadrats; B: sponge-dominated quadrats. Bars indicate standard deviation, dashed horizontal line represents zero (no effect of the predictor - growth form). The numbers indicate how many random iterations of the crossvalidation returned a non-zero value for the coefficient of that predictor. Only predictors whose coefficients were not shrunk to zero in $95 \%$ of the iterations were considered. $\mathrm{R}=$ rugosity (note the inverse scale), $1 / \mathrm{k}=$ vector dispersion, $\mathrm{D}_{1-5}=$ fractal dimension, $\mathrm{BRA}=$ branching, $\mathrm{TAB}=$ table, $\mathrm{FOL}=$ foliose, $\mathrm{MAS}=$ massive, $\mathrm{SUB}=$ sub-massive, $\mathrm{FRL}=$ free-living, $\mathrm{ENC}=$ encrusting, $\mathrm{TUB}=$ tubular, $\mathrm{REP}=$ repent, $\mathrm{DIG}=$ digitate, $\mathrm{BAR}=$ barrel, $\mathrm{CUS}=$ cushion, $\mathrm{GLO}$ $=$ globular.

Figure 6.1. Model domain of Atlantis Great Barrier Reef. Colours indicate depth of model boxes. White boxes indicate non-dynamic boundary boxes.

Figure 6.2. Data points from the Seabed Biodiversity Project (blue), and from the AIMS Longterm Monitoring Program (red), overlaid to the model domain of Atlantis Great Barrier Reef. 
Figure 6.3. Proportional cover of the main benthic groups in Atlantis Great Barrier Reef, as estimated by combining data from the Seabed Biodiversity Program (Pitcher et al. 2007) and the Long-term Monitoring Program from AIMS. Values indicate the 0-1 proportion of a box occupied by the group. White boxes are non-dynamic boundary boxes.

Figure 6.4. Proportional cover of heterotrophic, phototrophic, and bioeroding sponge in Atlantis Great Barrier Reef. Values refer to fraction of total box area occupied by a group. White boxes indicate non-dynamic boundary boxes. 176

Figure A1.1. Signed digraph of a predator (2) feeding on a prey item (1). Both variables are self-dampened. 250

Figure A2.1. Some common sponge taxa from the Hoga Island assemblage. Bars correspond to $1 \mathrm{~cm}$. A: Acanthella sp.; b: Agelas sp.; c: Axinella sp.; d: Axinyssa sp.; e: Chalinula milnei; f: Chalinula nematifera; g: Chalinula sp.; h: Chelonaplysilla sp. 1. 257

Figure A2.2. Scatterplot of the normalised residuals (left), histogram of the normalised residuals (middle), and quantile-quantile plots (right) of the Linear Mixed effects Models (LMMs) that showed significant fixed effects. a: sponge abundance $(\mathrm{N})$ as function of site; $b$ : species richness (S) as function of site, $\mathrm{N}$, and interaction between these; c: Shannon's diversity $\left(H^{\prime}\right)$ as function of site, $N$, and interaction between these; d: Simpson's evenness $\left(E_{1 / D}\right)$ as function of site, $\mathrm{N}$, and interaction between these. .262

Figure A2.3. Sea surface temperature (SST) from the Wakatobi Virtual Station between 2005 and 2017. Satellite data from the National Oceanic and Atmospheric Administration's Coral Reef Watch programme 2000, available at https://www.ospo.noaa.gov/data/cb/TS_vs/vs_ts_Wakatobi_Indonesia.txt)...... .263

Figure A2.4. Sea surface temperature anomalies (SSTANOM) from the Wakatobi Virtual Station between 2005 and 2017. Satellite data from the National Oceanic and Atmospheric Administration's Coral Reef Watch programme (NOAA 2000, available at https://www.ospo.noaa.gov/data/cb/TS_vs/vs_ts_Wakatobi_Indonesia.txt). .264

Figure A2.5. Mean abundance (sponge patches $\mathrm{m}^{-2}$ ) of the most common, taxonomically identified taxa at the Hoga Island site. Of the 30 taxa that, summed, comprised $>90 \%$ of the total sponge abundance in each photoquadrat, these 25 could be identified to genus level ( 25 
of the total 141). Note the different scales on y-axes. Error bars indicate standard error of the mean. Letters A, B and C indicate the three sites.

Figure A2.6. Non-metric multidimensional scaling (MDS) plot of the sponge assemblage at Hoga Island, split by survey year to aid visualisation.

Figure A3.1. Coefficient of variation (SD / mean) obtained by averaging over values of percentage cover that had been calculated for each benthic group in 8 iterations for each amount of CPCe points $(20,40,60,80,100,120$ random points per $25 \times 25 \mathrm{~cm}$ image). In each panel, horizontal lines indicate $\mathrm{CV}=1,0.5$, and 0.2 (grey, blue, and orange respectively); vertical dashed line indicates $80 \mathrm{CPCe}$ points / image (the chosen amount). The evaluation of CV per number of points was carried out for 3 different, randomly selected $25 \times 25$ images, indicated by letters $\mathrm{A}, \mathrm{B}$, and $\mathrm{C}$. .271

Figure A3.2. Scatterplot of the normalised residuals (left), histogram of the normalised residuals (middle), and quantile-quantile plots (right) of the Linear Mixed effects Models (LMMs) that showed significant fixed effects. a: coral \% cover as function of site; b: sponge $\%$ cover as function of site; $c$ : algae $\%$ cover as function of site; crustose coralline algae (CCA) $\%$ cover as function of site, year, and combination of the two; other invertebrates \% cover as function of site, year, and combination of the two.

Figure A3.3. Temporal dynamics of the benthic groups at the three Sites on the Buoy 3 reef. Lines in different colours represent the raw data from different quadrats. Red lines represent the mean effects estimated by the linear mixed -effects models (LMMs), dashed lines represent $95 \%$ confidence intervals of model estimates

Figure A3.4. Example of association between filamentous turfing algae and a calcareous sponge (Clathrina sp., white in between algal filaments) in one of the quadrats. 276 


\section{List of Tables}

Table 2.1. Response of the modelled functional groups to the applied press perturbations (first row). Responses are positive (+), negative (-), or ambiguous (?). For unambiguous responses, the weighted prediction ( $\mathrm{w}$ ) is indicated. $\mathrm{W}$ ranges from 0 to 1 , with $\mathrm{w}=0$ corresponding to complete ambiguity and $\mathrm{w}=1$ to complete certainty. A prediction weight of $\mathrm{w} \geq 0.5$ has been found to correspond to $>90 \%$ correct predictions (Dambacher et al. 2003a, see also Appendix 1).

Table 3.1. Coefficients of linear mixed effects models (LMMs). The fixed model term, coefficient estimate, SE, DF, t- and p-value are reported for all dependent variables (sponge abundance N, species richness S, Shannon's diversity H', and Simpson's evenness $E_{1 / D}$ ). Significant results $(\mathrm{p}<0.05)$ highlighted in bold.

Table 3.2. List of most common taxonomically identified sponge taxa from Hoga Island, Wakatobi, Indonesia. Of the sponge taxa that, summed, comprised $>90 \%$ of the total sponge abundance in each photoquadrat ( 30 of the total 141 taxa), the following 25 could be identified to genus level or lower. Abbreviations used in the Canonical Analysis of Principal coordinates (CAP) plot (Fig. 3.6-B) are reported in the taxon column. Mean abundances for this reef during the study period are reported. .86

Table 4.1. Groups of benthic organisms or substrata used in the percent cover analysis 105

Table 4.2. Results of the linear mixed-effects models (LMMs) showing the effects of site and year on percentage cover of the benthic taxonomic groups. Coefficient estimates, their standard error (SE), model degrees of freedom (DF), t-values, and p-values $(\mathrm{P})$ are reported for each fixed effect ( $\mathrm{P}$ values in bold are significant). Note that, for each model, the estimated Intercept refers to site $\mathrm{A}$, and the estimates for site $\mathrm{B}$ and site $\mathrm{C}$ indicate the difference between the Intercept at the respective sites and the intercept at site A. Similarly, the effect of year refers to site A, the estimates for the effect of year at site B and site C (indicated as [Year]:[Site]), indicate effect differences between the respective sites and site $\mathrm{A}$.

Table 6.1. Mapping of the benthic group in the AIMS Long-term Monitoring Program (LTMP) as Atlantis GBR groups.

Table 6.2. Values of maximum biomass $B_{\max }$ of the benthic groups in Atlantis Great Barrier Reef, the assumptions used to derive them, and the sources of the data utilised. 
Table 6.3. Main traits of sponge groups in Atlantis Great Barrier Reef, examples of the corresponding Atlantis parameters (see Audzijonyte et al. 2019 for the function of each parameter), and the sources used to parameterise them.

Table A2.1. List of the 42 taxonomically identified sponge taxa of the Hoga sponge assemblage. The remaining taxa were not possible to identify, largely due to lack of suitable samples or as a result of not being able to export specimens from Indonesia in many years of the survey. .255 
Chapter 1. General Introduction 


\subsection{Threats to coral reefs and coral decline}

Emissions of carbon dioxide have been steadily increasing after the Industrial Revolution, and concentrations of $\mathrm{CO}_{2}$ in the atmosphere have now passed $410 \mathrm{ppm}$, well above the preindustrial levels of $280 \mathrm{ppm}$. Increased atmospheric $\mathrm{CO}_{2}$ is causing increases in average seawater temperatures (ocean warming, $\mathrm{OW}$ ) and $p \mathrm{CO}_{2}$, with the latter resulting in decreased $\mathrm{pH}$ (ocean acidification, OA). The Intergovernmental Panel for Climate Change (IPCC) predicts mean increases in sea surface temperature of $1.1-4.0^{\circ} \mathrm{C}$ and a decrease in seawater $\mathrm{pH}$ of $0.2-0.32$ units by 2100 , depending on the levels of $\mathrm{CO}_{2}$ emissions in the next decades (IPCC, 2014).

Coral reefs are among the most productive and diverse ecosystems on Earth, supporting immense biodiversity and the livelihood of millions of people. However, coral reefs are threatened by anthropogenic OW and OA. An increase in water temperature causes a disruption of the symbiosis between corals and their associated photosynthetic algae. As a result, corals expel their photosymbionts, which causes coral bleaching and death in case of prolonged thermal stress. Large-scale bleaching events are occurring with increasing frequency due to increasing seawater temperatures (Hughes et al. 2017a, 2018), and projected levels of OW will have major impact on coral reefs globally in the near future (Hughes et al. 2017b). Moreover, $\mathrm{OA}$ and the corresponding undersaturation of carbonates make carbonate accretion more difficult, and contributes to the dissolution of existing carbonate structures (Feely et al. 2004, Orr et al. 2005). As a result, coral accretion is expected to be compromised on future reefs (Hoegh-Guldberg et al. 2007). Furthermore, storm intensity is predicted to increase globally as a result of climate change (Emanuel 2013), with projected severe ecological impacts on coral reefs (Cheal et al. 2017).

In addition to the global threat of climate change, local-scale disturbances exert increasing pressure on coral reefs in the Anthropocene, which is defined by human activities significantly influencing the planet (Crutzen 2006). For example, decreased water quality caused by land runoff has detrimental effects on coral physiology and diversity (e.g. Brown and Hamilton 2018, Lapointe et al. 2019), while overfishing of herbivorous fishes can facilitate an increase in macroalgal dominance on coral reefs (Mumby et al. 2007).

On coral reefs, corals are only one of the ecologically important organisms that may be affected by global climate change and local anthropogenic disturbances. Coral reef sponges can be an abundant component of the benthic community, their ecological importance is 
increasingly being recognised (see Pawlik and McMurray 2020). Because of their abundance and ecological importance, the way sponges respond to environmental change have been gaining increasing scientific attention.

\subsection{Sponges: evolution and taxonomy}

Sponges (phylum Porifera) are the most ancient multicellular metazoans to populate the oceans, with fossils dating back to the late Precambrian ( 580 million years ago, Li et al. 1998). Sponges are sessile benthic organisms that have no organ or tissue differentiation, with a body plan consisting of an undifferentiated mesohyl contained between two single layers of cells, an external pinacoderm and an internal choanoderm. The choanoderm is composed of flagellated cells, the choanocytes, whose function is to pump water into an internal aquiferous system for water filtering (de Vos et al. 1991). Water enters this system of channels through microscopic inhalant openings, the ostia, and exits it through exhaling venting oscula. With such a simple body plan, sponges have remained virtually unchanged throughout their entire evolutionary history (Wood 1995), colonizing almost all aquatic environments, both freshwater and the oceans, at all latitudes and at a range of depths (e.g. McClintock et al. 2005, Manconi and Pronzato 2008, Bell et al. 2015a). Four classes of marine sponges are accepted within the phylum Porifera: Demospongiae, Calcarea, Hexactinellida, and Homoscleromorpha, and over 9000 sponge species have been described and accepted as of 2019 (van Soest et al. 2019).

With the exception of carnivorous sponges (Vacelet and Boury-Esnault, 1995), all sponges are suspension feeders that filter water to retain food particles. However, in shallow and nutrient-poor waters, many sponge species also rely on photosymbionts to supplement their heterotrophic diet (reviewed in Wulff 2006a). Wilkinson (1983) estimated that 9 out of 10 of the most abundant sponge species on the Australian Great Barrier Reef host cyanobacteria, whereas between $28-58 \%$ of sponge species on Caribbean coral reefs host photosymbionts (Wilkinson 1987, Wilkinson and Cheshire 1990). Most commonly, phototrophic sponges host cyanobacteria, but some bioeroding taxa host zooxanthellae of the genus Symbiodinium, like corals (Pang 1973).

Sponges are challenging organisms to study and monitor. They are a very diverse phylum (Hooper et al. 1999), and in situ identification is often difficult due to their high morphological plasticity and due to the abundance of cryptic species (Shaffer et al. 2018). Moreover, sponges are not easily kept in aquaria (Wulff 2017), and sponge taxonomy is still developing and rapidly 
changing (e.g. Gazave et al. 2012, Morrow and Cárdenas 2015). Sponges are also difficult to monitor, because life history traits vary broadly between species, and because sponges have plastic body plans capable of fragmentation and fusion (reviewed in Bell et al. 2017). These factors have contributed to many monitoring programmes excluding sponges (Wulff 2001). However, sponges have many ecological roles, which mediate the ecological importance of this phylum across coral reefs and marine ecosystems in general (reviewed in Wulff 2006a, Bell 2008).

\subsection{Functional roles of sponges on coral reefs}

\subsubsection{Filtration, pumping, and biogeochemical cycling}

Sponges remove organic matter from the water column and make it available for higher trophic levels, contributing with other organisms to the removal of particulate and dissolved organic matter from the water column (Bell 2008). As benthic suspension feeders, sponges pump and filter large volumes of water to retain ultraplankton and picoplankton (Reiswig 1971a, b). If this water filtration system is disrupted, for instance as a result of sponge mass mortalities, organic matter may accumulate in the water column, with detrimental effects for the ecosystem (Wulff 2001).

Sponges also actively feed on dissolved organic carbon, or DOC (Yahel et al. 2003, Maldonado et al. 2012), although with species-specific efficiency (Hoer et al. 2018). Recent studies have shown that many sponges transform the carbon that they assimilate as DOC into particulate detritus, which originates from fast cell turnover inside the sponge (de Goeij et al. 2008, Rix et al., 2016, 2017, 2018). The carbon remineralised this way is consumed by detritivores, thus re-entering the ecosystem, completing the cycle defined as the 'sponge loop' (de Goeij et al. 2013). The sponge loop is thought to be an important pathway of carbon cycling on coral reefs, which are generally oligotrophic aquatic environments (de Goeij et al. 2013, Pawlik and McMurray 2020).

By filtering water, sponges also have a major role in elemental cycles on coral reefs. Most sponges use silicon to build spicules. Although diatoms are possibly the main agents of silicon cycling on a global scale (Greenwood et al. 2001, Rickert et al. 2002), sponges are thought to be important silicon sinks, due to their longer life span compared to diatoms and to the slower dissolution of sponge spicules than diatom frustules after death (Maldonado et al. 2005, 2010). Although the available information is limited, sponges also take part to the recycling of 
nitrogen and phosphorous on coral reefs. Both particulate organic nitrogen (PON) from picoplankton and dissolved organic nitrogen (DON) are consumed by coral reef sponges (Hadas et al. 2009, de Goeij et al. 2013), which then produce ammonium $\left(\mathrm{NH}_{4}{ }^{+}\right)$as a metabolic waste product (Diaz and Ward 1997). Sponge-associated bacterial communities hosted by coral reef sponges are also linked to nitrification (Diaz and Ward 1997, Archer et al. 2017), nitrogen fixation (Zhang et al. 2014), and sequestration of phosphorus (Zhang et al. 2015, Archer et al. 2017).

\subsubsection{Reef building and degradation}

Sponges can contribute to the formation of habitat, especially when found in dense aggregations (reviewed in Maldonado et al. 2016). For example, deep-sea sponge grounds constitute complex habitats (Buhl-Mortensen et al. 2010) and can be found across latitudes, from the North Atlantic (Hogg et al. 2010) to the North Pacific (Conway et al. 2001) and the Australian continental shelf (Fromont et al. 2012). The role of sponges as reef builders in shallow habitats has received limited attention (but see below for the use of sponges as microhabitat for associated fauna).

On coral reefs, sponges can have either positive or negative effects on the accretion-erosion balance of reefs. Most sponge species do not produce calcareous skeletons like corals, and therefore do not contribute to net reef accretion (Rützler 2004). Sponges can bind coral rubble together, thus aiding the stabilisation of coral reefs after destructive events (Wulff and Buss 1979, Wulff 1984). However, sponges, are also conspicuous bioeroders on coral reefs (Goreau and Hartman 1966, MacGeanchy 1977). Bioerosion is the degradation of consolidated carbonates into smaller fragments and then into sediments by chemical coring (Rützler 1975), and if it exceeds the accretion of new carbonate from corals and other biocalcifiers it can result in the degradation of reef structure (Stearn et al. 1977). Clionid sponges, for example, excavate the base of large coral colonies, causing them to break down and be dislodged (Rützler 2004). Sponge bioerosion is an important ecological process on coral reefs (reviewed in Schönberg et al. 2017), and while sponge-mediated rubble-binding can have positive effects on reef recovery, sponges have an overall negative effect on the accretion-erosion balance of coral reefs (see Pawlik and McMurray 2020).

\subsubsection{Biological interactions of sponges}

Sponges on coral reefs interact with a broad range of organisms. Sponges must compete for space with other benthic organisms, including other sponges, corals, macroalgae and other 
benthic invertebrates. While not many sponge species regularly outcompete corals (Rossi et al. 2015), some sponges can overgrow and smother corals (Coles and Bolick 2007, Loh et al. 2015, Elliott et al. 2016). Some sponge species produce secondary metabolites that have harmful effects on other benthic competitors (Pawlik et al. 2007). Allelochemical-mediated overgrowth has been reported for sponges outcompeting neighbouring bryozoans (Jackson and Buss 1975, Buss 1976), corals (Porter and Targett 1988, de Voogd et al. 2004) and other sponges (Turon et al. 1996, Thacker et al. 1998). Competitive interactions between sponges and algae on coral reefs have received limited attention. On Caribbean reefs, macroalgae can curb the growth of Clionid sponges by direct size-dependent competition and pre-emption of free substrate (González-Rivero et al. 2012, 2016), and by limiting the advance of excavating sponges into substrates overgrown by algae (López-Victoria et al. 2006). Contact with macroalgae can also have negative physiological effects on non-excavating sponges (Easson et al. 2014).

Sponges are grazed by invertebrate and vertebrate predators. Invertebrate sponge predators are common in temperate and tropical seas, and include molluscs (e.g. Gemballa and Schermutzki 2004, Pawlik and Deignan 2015), echinoderms (e.g. McClintock et al. 2005), and crustaceans (Guida 1976). On coral reefs, vertebrate spongivores are abundant and diverse (reviewed in Wulff 2006a). Hawksbill turtles are specialised spongivores (Meylan 1988), whereas opportunistic feeding is observed in several fish species (Dunlap and Pawlik 1996, Wulff 1997). While sponge assemblages are rarely top-down controlled in cold and temperate waters (Wulff 2006a), predation is thought to be a primary driver of sponges on Caribbean coral reefs (reviewed in Pawlik et al. 2018). On Caribbean reefs, DOC-consuming sponges convert DOC into sponge biomass, which is eaten by spongivorous fishes (McMurray et al. 2018). This pathway mediates the reintroduction of DOC into the reef food web as an alternative to the sponge loop (McMurray et al. 2018), and it underpins the ecological importance of spongivory on Caribbean reefs (Pawlik and McMurray 2020).

Little is known about spongivory in the tropical Indo-Pacific. Fishes and opistobranch molluscs were found to have trophic interactions with sponges, but the role of these predators in controlling sponge assemblages remains unclear (e.g. Powell et al. 2015).

\subsubsection{Sponges as providers of habitat}

Sponges can form important associations with macrofauna and microorganisms. For example, deep sea sponge aggregations offer habitat for marine fauna (reviewed in Buhl- 
Mortensen et al. 2010), and provide structural complexity that is associated with invertebrate diversity (Beazley et al. 2013) and fish survival to predation (Ryer et al. 2004). Furthermore, sponges with upright morphologies provide habitat for fishes in estuarine systems (van Lier et al. 2017). Sponges also host diverse endobiotic invertebrate communities within their aquiferous system (Koukouras et al. 1996, Gerovasileiou et al. 2016, Papatheodoulou et al. 2019), and they provide habitat for complex communities of microorganisms that mediate nutrient cycling and photosynthesis (reviewed in Taylor et al. 2007)

The role of sponges as ecosystem engineers on coral reefs has not been investigated yet, and hard corals are considered the primary biogenic contributors to reef structure (Wild et al. 2011). Coral reef sponges provide refugia for juvenile lobsters in the Caribbean (Herrnkind et al. 1997), and may be offer habitat in degraded reef systems (Seemann et al. 2018), but their potential to offer reef structural complexity has not been explored yet.

\subsection{Examples of drivers influencing marine sponges}

Sponges and the functional roles they provide are affected by a complex interplay of physical drivers and biotic interactions. For example, water temperature is known to impact sponge physiology and ecology in many ways. Even short-term exposure to increased water temperature can cause higher respiration rates (e.g. Cheshire et al. 1995, 1997, Zocchi et al. 2003), mortality of the photosymbionts that can result in sponge bleaching (Vicente 1990, López-Legentil et al. 2008, McMurray et al. 2011), alterations to reproduction and larval dispersal (Cardone et al. 2008, Whalan et al. 2008), and reduced feeding (Massaro et al. 2012). Furthermore, light is a primary driver for sponge assemblages with a high proportion of phototrophic species like the ones on the Great Barrier Reef (e.g. Cheshire and Wilkinson 1991). Sedimentation can also impact sponge diversity and abundance in a number of ways (see Bell et al. 2015b), and while some sponges can tolerate highly sedimented environments (Biggerstaff et al. 2017a), high sedimentation can cause increased metabolic rates for coral reef sponges (McGrath et al. 2017).

Sponge assemblages are also influenced by local-scale environmental conditions. For example, water flow and substrate heterogeneity drive sponge morphological diversity in the Indian Ocean (Barnes and Bell 2002), and substrate inclination is an important determinant of sponge abundance and diversity in temperate systems (Bell and Barnes 2000a, b). 
Biotic interactions are also important drivers of sponge assemblages on coral reefs. Research on the relative roles of bottom-up versus top-down controls on tropical sponges has mostly focused on Caribbean reefs. There is ongoing debate around whether Caribbean sponge assemblages are driven primarily by top-down (i.e. predation from sponge eaters, reviewed in Pawlik et al. 2018) or bottom-up controls (i.e. food limitation, Lesser and Slattery 2013, Slattery and Lesser 2015). Recent findings suggested that a combination of top-down and bottom-up controls may regulate sponge assemblages in the Caribbean (Wulff 2017), but these findings may be restricted to lagoonal habitats and not apply to fore-reefs (Pawlik and McMurray 2020).

In the context of rapidly changing oceans, investigating how physical and biological drivers affect sponges on coral reefs is gaining increasing attention.

\subsection{Regime shifts towards sponge-dominated reefs}

Sudden changes in an ecosystem may result in a regime shift towards an alternative stable state (Scheffer et al. 2001). With corals declining as a result of global environmental change and increasing local anthropogenic pressure, coral reefs may shift towards alternative states in which other benthic organisms become dominant (Hughes 1994). Persistent anthropogenic perturbations can reduce the resilience of coral reefs to catastrophic events and contribute to the establishment of alternative stable states (Dudgeon et al. 2010). However, the establishment of ecological feedback is necessary for regime shifts to be permanent, as these feedbacks prevent the ecosystem from reverting to its original state (Suding et al. 2004). Feedbacks that are known to reinforce a macroalgal state include inhibition of coral growth and recruitment from macroalgae (Tanner 1995, Johns et al. 2018), and a decoupling between grazing pressure of herbivorous fishes and macroalgal abundance (Williams et al. 2001, Mumby et al. 2007). Regime shifts towards macroalgal systems are the most widely documented on coral reefs (e.g. Hughes 1994, Mumby et al. 2007, Bruno et al. 2009, Jouffray et al. 2015). However, reefs may also shift to states dominated by other organisms like urchins, soft corals and sponges, depending on the drivers of the change and on the feedback processes that may be established (Norström et al. 2009).

Recent research has shown that some sponge species may be more tolerant than corals to OW and OA (reviewed in Bell et al. 2018b). For example, although increased temperature can cause metabolic stress and bleaching in coral reef sponges (see above), sponges have been found to 
be more resistant than corals to thermal stress (e.g. Schönberg and Suwa 2007, López-Legentil et al. 2008). Furthermore, because most sponges do not build carbonate skeletons, sponges may also be more tolerant than corals to a decrease in water $\mathrm{pH}$, notwithstanding the potential negative effects of hypercapnia on sponge physiology (Pörtner et al. 2004). Although the impacts of acidification on sponges and their functions are still poorly understood (Stubler et al. 2015), some phototrophic sponges may benefit from increased seawater $p \mathrm{CO}_{2}$. For example, sponges near a $\mathrm{CO}_{2}$ "seep" in Papua New Guinea are $~ 40$ times more abundant and hosted more photosymbionts than those distant from the seep, whereas corals are less abundant and hosted less photosymbionts (Morrow et al. 2015). Moreover, an increase in $p \mathrm{CO}_{2}$ was also found to mitigate the effects of warming on phototrophic sponge species on the Great Barrier Reef, although it exacerbated them on heterotrophic species (Bennett et al. 2017).

Some coral reefs have already experienced transitions towards sponge-dominated states. Sponge abundance has increased on coral reefs in the Caribbean (Zea 1993, McMurray et al. 2010, Colvard and Edmunds 2011, Villamizar et al. 2014), where sponges are now considered the main habitat-forming organisms (Loh and Pawlik 2014). El Niño Southern Oscillation (ENSO) events have caused a decline in coral cover in Bahia (Brazil), followed by an increase in sponge density (Kelmo et al. 2013, 2014). Increased sponge dominance has also been observed at some reefs in the Indo-Pacific, though habitat degradation and sedimentation, instead of warmer water, were the likely drivers of the shifts (Bell et al. 2015b). For example, the encrusting phototrophic sponge Lamellodysidea herbacea has increased at a sedimented site in the Wakatobi Marine Park in Indonesia (Biggerstaff et al. 2017b). Similarly, degraded reefs at the Palmyra Atoll are thought to have shifted from coral- to sponge-dominated systems in the recent past (Knapp et al. 2013, Knapp et al. 2016), and volcanic ashes suspended in the water column were associated with sponge population expansions in the Northern Mariana Islands (Schils 2012).

Shifts towards sponge dominance have not been ubiquitous on coral reefs so far (Pawlik and McMurray 2020). However, as a result of the recent reports of increased sponge abundance and the evidence of higher tolerance of sponges to climate change conditions compared to corals, sponges have been proposed as potential 'winners' on future coral reefs (Bell et al. 2013, 2018b).

Potential direct effects of increased sponge dominance on future reefs include enhanced sponge-mediated carbon fluxes (McMurray et al. 2017) and increased bioerosion (Fang et al. (2013), but see Ramsby et al. (2018) for evidence that bioeroding sponges may not tolerate 
future warming). However, while the ecological functions of reefs that transition to a macroalgal-dominated state have recently been discussed (Fulton et al. 2019), the ecosystemlevel effects of increased sponge dominance on future reefs are currently unknown. As coral reefs transition towards new configurations in the Anthropocene (Hughes et al. 2017b), it is critical to understand the ecological functions of altered reef states, in order to better predict how reef ecosystems will change in the future and to apply appropriate management strategies (Graham et al. 2014). Ecological models can be used as means to make such predictions, when they are informed by the knowledge of the key ecological processes in the studied system.

\subsection{Ecological modelling of coral reef sponges}

Coral reefs are complex ecosystems, influenced by many physical drivers. Predicting the ecosystem-level effects of perturbations on coral reefs is difficult and prone to uncertainty (Harborne et al. 2017). Ecological models (see Jopp et al. 2011 for an introduction) facilitate the representation of natural systems, the understanding of their dynamics, and the projection of their future states. Ecological models span in complexity from simple qualitative models that help develop research hypotheses to high-complexity, fully quantitative frameworks that capture entire ecosystems. Qualitative models focus on the key components and processes of the ecosystem, and they can give qualitative insights on the effects of a disturbance, such as the impact of OW and OA on coral reefs. Qualitative models are useful to develop testable hypotheses, identify gaps in the knowledge of the system, and corroborate quantitative studies (see Dambacher et al. 2009). Qualitative models used in ecology include the qualitative analysis of the community matrix, and of the ecological feedbacks that are established when the equilibrium of the modelled community is altered (Puccia and Levins 1985). Complex quantitative models, like ecosystem models, capture many interacting species, flows of energy and matter between them, environmental drivers, and economic and social components. Ecosystem models are used for the holistic representation of natural systems, for the simulation of future scenarios of environmental change and human activities, and for the evaluation of management strategies (see Fulton et al. 2011).

One of the most flexible and sophisticated ecosystem models currently available is Atlantis (Fulton et al. 2011). Atlantis allows the dynamic modelling of the biological components of an ecosystem, the biophysical drivers affecting them, human uses of the system, and adaptive management strategies (Audzijonyte et al. 2019). Atlantis is a largely deterministic spatially- 
explicit model, with the modelled area partitioned into a mosaic of polygons that are defined after biotic, physical, and management-driven rationales. Energy and matter are exchanged between the polygons, and all model components are dynamically coupled. Atlantis has been used to model future scenarios of environmental change and fisheries with the aim to aid Ecosystem-Based Management (EBM, Link et al. 2010), with over 30 applications (Weijerman et al. 2016) worldwide. Atlantis applications range from relatively small islands like Guam (Weijerman et al., 2014) to large biogeographic regions like the Northeast United States (Link et al., 2010).

Conceptual models have been used to summarise trophic interactions between sponges and their predators (Pawlik 2011) and their food sources (de Goeij et al. 2013), whereas parameterised, state transition models have been used to predict the effect of sponge demographics on sponge-mediated carbon fluxes (McMurray et al. 2017), and the response of bioeroding sponges to coral bleaching (Chaves-Fonnegra et al. 2017). Species distribution models have also been applied to predict the spatial distribution of sponge assemblages as a function of physical variables (Huang et al. 2011), and one ordinary differential equation model has been used to explore three-way competition between corals, macroalgae and sponges on Caribbean reefs (González-Rivero et al. 2011). In addition, food web models have captured some important ecological roles of sponges, like the sponge loop and its effect on food webs of reefs shifting towards algal dominance (Silveira et al. 2015). However, sponges have been underrepresented in more complex ecosystem models so far.

The application of ecosystem models like Atlantis requires high volumes of data, and the quantitative knowledge of the modelled processes. Several aspects of coral reef sponge ecology, however, are still poorly understood. For example, the magnitude and ubiquity of some sponge-mediated ecological processes, like the sponge loop and the contribution to reef structural complexity, are still unclear (see Pawlik and McMurray 2020). In addition, dynamic ecosystem models must be grounded in the knowledge of how the modelled communities develop over time. However, with the exception of the Caribbean (Pawlik and McMurray 2020), temporal variability of multi-species sponge assemblages has received limited attention, partially due to the restrictions to sponge monitoring discussed above. Furthermore, only recently it was possible to elucidate some of the mechanisms that mediate sponge response to OW and OA (Bennett et al. 2018), posing the problem of correctly modelling how sponges may respond to the simulated disturbances. These factors have limited the accurate representation of sponges and their ecological roles in in ecosystem models so far. 
In ecosystem models for coral reef systems, sponges have been pooled with other benthic invertebrates into generic benthic filter feeders (e.g. Weijerman et al. 2014). However, spongemediated ecological processes influence the functions of coral reefs (Bell et al. 2013, Pawlik and McMurray 2020), and may become even more important if sponge abundance increases on future reefs (McMurray et al. 2017), and should therefore be explicitly represented in ecosystems models of coral reefs. In addition, the differentiation of phototrophic and heterotrophic sponges is desirable when capturing sponges in an ecosystem model, because these two trophic groups have different ecological roles (Bell 2008), and can respond in different ways to environmental change (Bennett et al. 2017).

\subsection{Scope of this thesis and specific aims}

Sponges are an important component of coral reef ecosystems. Their ecological relevance is often overlooked in scientific research, and this can limit the understanding of coral reefs and their dynamics. Given the potential role of sponges as some of the 'winners' in response to climate change and the potential future increase in sponge dominance, it will be crucial to understand how sponge-dominated reefs may function, how coral reef trophodynamics will be altered, and ultimately how the human use of coral reefs will be affected. Ecosystem models can provide insights into present and future dynamics of sponge assemblages and coral reefs in general, pending the appropriate representation of sponges in such models, the interacting components of the system and the drivers behind their dynamics.

The overarching goals of this thesis are: 1) to identify and address some important gaps that limit our understanding of how sponge-dominated reefs may function; and 2) the explicit representation of sponges in ecosystem models for coral reefs. The specific aims of this thesis, each corresponding to one Chapter, are:

1. To develop a qualitative mathematical model of a coral reef, and to use this model to obtain qualitative predictions of the ecosystem-level effects of a regime shift towards a sponge-dominated reef. The analysis of model uncertainty will help to identify some aspects of sponge ecology that must be clarified to better predict how sponge-dominated reefs may function.

2. To quantify the temporal variability of a coral reef sponge assemblage in the Wakatobi Marine Park (Indonesia). This Chapter will elucidate how sponge abundance and biodiversity 
can change over time on coral reefs, which is useful when predicting how rapidly sponge assemblages may develop after a decline in corals.

3. To determine long-term dynamics and interannual variability of the benthic community at the same Wakatobi site. This Chapter will quantify the temporal variability of all benthic sessile organisms and the effects of available substrate on benthic cover, providing insights into how changes in benthic dominance may occur on future reefs after coral mortality.

4. To determine the role of sponges as providers of reef structural complexity on the same Indonesian reef system, using 3D photogrammetry. By comparing the 3D structure of spongedominated and coral-dominated reef areas, and by determining which sponge morphologies contribute to reef structural complexity, this Chapter will investigate whether spongedominated reefs may be complex habitats comparable to coral-dominated reefs.

5. To develop a set of novel components of the Atlantis ecosystem model, dedicated to the detailed representation of sponges, their ecological roles, and their responses to environmental drivers. These components consist of additions and modifications to the Atlantis framework, and they include: the explicit modelling of phototrophic, heterotrophic, and bioeroding sponges, and of spongivorous fishes; a sub-model for competition for space between corals, macroalgae and sponges; a sub-model for sponge bioerosion damaging reef rugosity; and silicon limitation to sponge growth. These new model components are informed by the findings of the previous chapters, and by extensive review of the current knowledge of sponge ecology and ecophysiology. 


\section{Chapter 2. Qualitative modelling of alternative reef states}

The models and model results presented in this chapter are published in: Bell, J. J., Rovellini, A., Davy, S. K., Taylor, M. W., Fulton, E. A., Dunn, M. R., Bennet, H. M., Kandler, N. M., Luter, H. M., and Webster, N. S. (2018). Climate change alterations to ecosystem dominance: how might sponge-dominated reefs function? Ecology, 99(9):1920-1931. https://doi.org/10.1002/ecy.2446. Reprinted with permission (altered version). John Wiley and Sons is the copyright holder for this article. 


\begin{abstract}
Coral reefs in the Anthropocene are facing the threat of global climate change combined with other local-scale anthropogenic pressures. Corals are vulnerable to ocean warming (OW), ocean acidification (OA) and decreased water quality, and have been declining globally. As some coral reef sponges have shown higher tolerance than corals to OW and OA, it has been proposed that sponges may have an advantage over corals in a changing climate, and that sponge dominance may increase on future coral reefs. Understanding the ecological functioning of alternative states of coral reefs is critical to predict how future reef ecosystems will function, but the cascading effects of a shift towards sponge dominance are currently unknown. In this chapter, I used qualitative modelling to explore the ecosystem-level effects of environmental change on a coral reef ecosystem, and to identify the key ecological processes that determine how a sponge reef could function. A qualitative model (QM) of a coral reef was used to predict ecosystem-level responses to increased temperature, acidity, and turbidity. A set of lower-complexity QMs capturing key ecological processes (structural complexity, the sponge loop, and competitive superiority of macroalgae), were then used to obtain qualitative predictions of the effects of a decline of corals and an increase in sponge abundance on the reef. Model predictions showed that simulated OW, OA, and decreased water quality had negative effects on corals. Such coral decline was followed by a loss of reef structural complexity, increased sponge and macroalgal abundance, enhanced secondary productivity, and boosted detrital pathways. Analysis of the uncertainty around model predictions highlighted that a better understanding of the temporal variability of sponges and their contribution to reef structural complexity will provide better insights on how sponge reefs may function.
\end{abstract}

\title{
2.1. Introduction
}

Many terrestrial, freshwater and marine ecosystems have been degraded across the world in recent decades as a result of global environmental change and local anthropogenic pressure. On coral reefs, ongoing global decline of corals has been caused by increased water temperature (Graham et al. 2015, Hughes et al. 2017a), cyclones (Cheal et al. 2017), and pollution (Brown and Hamilton 2018). With the levels of ocean warming (OW) and acidification (OA) currently projected for the near future, corals are predicted to keep declining (Hoegh-Guldberg et al. 2007, Hughes et al. 2017b), to the detriment of coral reef biodiversity 
and productivity. As a result of declines in coral, it is likely that future coral reefs will assume new configurations that will function differently from past and current reefs (Hughes 2017b). The ecological functions of these reef states with low coral cover, however, are currently poorly understood, because effects of changes in organism abundance on ecosystem processes are often difficult to quantify (see McMurray et al. 2017). However, understanding the ecological functions of coral reefs is critical to their management under the increasing threats that these ecosystems are facing (Bellwood et al. 2019). Therefore, it is important to understand how known ecological processes will be altered on low-coral cover reef configurations (Graham et al. 2014, Harborne et al. 2017).

A widely documented outcome of coral loss driven by environmental change is a shift of the reef system towards macroalgal dominance (Dudgeon et al. 2010). Regime shifts of coral reefs towards macroalgal-dominated states have been reported from many reefs globally (e.g. Hughes 1994, Mumby et al 2007, Bruno et al. 2009, Jouffray et al. 2015), and the ecological functioning of these macroalgal states has been recently reviewed (Fulton et al. 2019). While algal-dominated reefs are the most often documented alternative states, shifts towards states dominated by other organisms have also been observed on some coral reefs (Norström et al. 2009). For example, a transition towards sponge-dominated reefs may occur if sponge abundance increases over time (Bell et al. 2013, 2018b). Some coral reef sponges are more tolerant than corals to the combined effects of ocean warming and acidification (Duckworth et al. 2012, Bennett et al. 2018), and increased sponge abundance has been reported from some coral reefs where corals have declined (McMurray et al 2010, Schils 2012, Kelmo et al. 2013, Knapp et al. 2013, 2016, Powell et al. 2014, Biggerstaff et al. 2017b). However, sponges are still affected negatively by an increase in seawater temperature (Bennett et al. 2017, Ramsby 2018), and it is unclear whether increased sponge dominance will be ubiquitous on future coral reefs (Pawlik and McMurray 2020).

Sponges have many important ecological roles on coral reefs (see Chapter 1). These roles include the removal of carbon from the water column (Reiswig 1971a, 1975), the consumption of dissolved organic matter and production of sponge detritus for deposit feeders to consume via the 'sponge loop' (de Goeij et al. 2013, Rix et al. 2018), the provision of food for predators (McMurray et al. 2018), bioerosion of carbonates (Schönberg et al. 2017), competition for substrate with other sessile benthic organisms (Wulff 2006a), primary production (Wilkinson 1983), and the provision of habitat for associated microbiomes (Taylor et al. 2007). Due to the many functional roles of sponges, an increase in sponge abundance on coral reefs will have 
potential flow-on effects on the ecosystem. For example, increased sponge abundance may result into reduced substrate availability for other benthic organisms, with detrimental effects for weaker benthic competitors (de Voogd et al. 2004); furthermore, sponge-mediated carbon fluxes depend on sponge demographics in the Caribbean (McMurray et al. 2017). Moreover, some sponge-mediated ecological processes are expected to be enhanced under future environmental conditions. For example, bioerosion rates are likely to increase with future OA (Schönberg et al. 2017). However, the ecosystem-level consequences of changes in sponge abundance remain largely unknown (McMurray et al. 2017), and therefore little is currently known about how sponge-dominated reefs might function.

Coral reefs are complex and highly diverse ecosystems, and they are influenced by a large number of environmental drivers that affect many organisms and ecological processes. Therefore, quantifying the ecosystem-level effects of changes in benthic dominance is difficult. Qualitative modelling (Puccia and Levins 1985) is a mathematical modelling approach that allows natural system to be represented as a set of variables (like physical drivers, nutrients, biomass pools, etc.) linked by interactions (like predation, competition, mutualism, etc.). Qualitative models (QMs in the following text) use loop analysis (Levins 1974, Puccia and Levins 1985) to obtain qualitative predictions of how the modelled system responds when a perturbation affects one, or many, variables (Dambacher et al. 2002, Dambacher et al. 2003a). Qualitative modelling does not quantify the magnitude of the ecosystem responses to a perturbation, like quantitative models do. However, quantitative approaches normally require prior empirical observation, large volumes of data, and a quantitative knowledge of the ecological processes at play (Dambacher et al. 2009). QMs are useful to predict in which direction each component of the system will change when a perturbation is applied, with mathematical rigor and with an estimation of the uncertainty around the predictions.

Determining the uncertainty around model predictions is an important step of qualitative modelling (Dambacher 2002). This step allows the identification of potential gaps in our knowledge of how the system functions. Uncertainty in the predictions of QMs increases with system complexity, which is a function of the number of variables and links between them (Dambacher et al. 2002). For this reason, when representing a complex system like a coral reef, it is possible to use a set of alternative models of intermediate complexity capturing different aspects of the ecological system, and compare the predictions from these models (Dambacher et al. 2002, 2003a). This approach maintains system complexity on tractable levels, and it allows comparisons between the predictions from different models, thus aiding in identifying 
the key ecological processes to be captured in the representation of the system. This makes QMs useful tools to inform more complex quantitative models (Dambacher et al. 2009).

QMs of coral reefs have been used previously to review current knowledge of key processes of reef ecology while exploring the impacts of climate-related stress (see Mumby and van Woesik 2014, Harborne et al. 2017), to assess socioeconomic impacts of human activities on coral reefs (Dambacher et al. 2007, 2015), to support monitoring programs (Dambacher et al. 2013), and to explore the combined effects of anthropogenic and environmental factors on species of interest (Babcock et al. 2016). Previous quantitative (McMurray et al. 2017) and conceptual (Pawlik 2011, Pawlik et al. 2016) models have focused on sponge trophic interactions, and one model has explored three-way benthic competition between corals, sponges and macroalgae, with frequent positive outcomes for the latter (González-Rivero et al. 2011). However, no studies have yet investigated the ecosystem-level effects of an increase of sponge abundance on coral reefs (but see Bell et al. 2013 for discussion of the concept).

In this Chapter, I explored the cascading effects of an environmental change-driven shift from coral to sponge dominance on a coral reef ecosystem using a set of QMs. I then quantified the uncertainty around the predictions of these models, in doing so identifying some important gaps in the current knowledge of sponge ecology that limit the understanding of how reefs may respond to an increase in sponge abundance. The specific aims of this Chapter were: 1) to use a QM of a coral reef to explore the ecosystem-level effects of an increase in temperature, acidity and turbidity affecting the benthic community; 2) to use a simplified version of this QM to explore the effects of perturbation-driven decline of corals and increase of sponge abundance; 3) to evaluate the response of the ecosystem to a shift in benthic dominance when three important aspects of coral reef ecology (reef structural complexity, the sponge loop, and competitive superiority of macroalgae) were captured by a set of alternative QMs. These QMs emphasised the nodal ecological processes that may define the functioning of a spongedominated reef, and guided the focus of other Chapters in this thesis by highlighting some key questions that will help better understand the role of sponges on future reefs. In addition, the qualitative models developed in this chapter guided the modelling of sponges in the quantitative ecosystem model Atlantis (Chapter 6). 


\subsection{Methods}

\subsubsection{Qualitative modelling of natural systems: an overview}

A brief overview of the qualitative modelling approach is presented here (see Appendix 1 and references here for more detailed presentation of the approach). Qualitative modelling (Puccia and Levins 1985) aims to represent a complex system as set of components (or variables, e.g. populations, stocks, nutrient pools etc.) and the interactions between them, with no quantitative specification. The goal of the approach is to predict how a perturbation acting on one (or many) components propagates across the system, or in other words how all variables will qualitatively respond to a change in one variable (see Dambacher et al. 2002, 2003a, 2003b). QMs capture the key components of the system (or the components of interest in the investigation) and their dynamic relationships. QMs are useful to predict system behaviour in response to perturbations and to formulate hypotheses, particularly when: 1) the interactions between individual components of the system are known but difficult to quantify (for example due to a lack of data, Dambacher et al. 2003a, b); and 2) the ecosystem-level effects of a perturbation that acts on one system component are unknown.

The first step of qualitative modelling is to select of the components and properties of the natural system that must be represented. High model complexity (i.e. many variables and links) comes at the cost of interpretation of model predictions (Dambacher et al. 2002), and therefore the goal is to build a parsimonious model that includes only the variables and the interactions that are of principal interest in the investigation at hand, while still capturing the key properties of the modelled system.

Qualitative modelling is based on the study of the qualitatively specified community matrix A, also known as a Jacobian matrix (Levins, 1968). A contains information on how the variables in the system interact with each other. For a system with 3 variables, $\mathbf{A}$ has the generic form:

$$
\boldsymbol{A}=\left[\begin{array}{lll}
a_{1,1} & a_{1,2} & a_{1,3} \\
a_{2,1} & a_{2,2} & a_{2,3} \\
a_{3,1} & a_{3,2} & a_{3,3}
\end{array}\right]
$$

Each element $\alpha_{i, j}$ in the community matrix details the effect that variable $j$ has on variable $i$. Such effect can be positive (+), negative (-), or absent (0). The community matrix has a graphical representation in a signed digraph (Fig. 2.1 for a 2-variable system), where system variables are represented by circles, and interactions by arrows. The arrowheads indicate the 
effect of the source variable on the target variable, with $(\rightarrow)$ denoting a positive effect, $(-\circ)$ a negative effect, and (-) no effect. Different combinations of these arrowheads can represent all pairwise biological interactions, for example predation $(\circ \rightarrow)$, competition $(\circ-\circ)$, and mutualism $(\leftarrow \rightarrow)$.

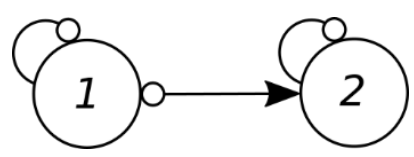

Figure 2.1. Signed digraph of a predator (2) feeding on a prey item (1). Both variables are self-dampened, meaning they have a negative self-effect (see text).

After the QM for the system (i.e. the community matrix) has been algebraically defined, but before it can be used to evaluate the response to perturbations, model stability must be evaluated. Qualitative modelling assumes that the modelled system is stable, or at equilibrium; that is, the system can return to its original state after a small and sudden pulse perturbation acts on it (Dambacher et al. 2003b). In mathematical terms, this is called Lyapunov stability (see Logofet 1993), and it is a function of the feedbacks (i.e. the pathways that connect the model variables) that are present in the system. From an ecological perspective, feedbacks determine how a perturbation propagates through the system, because they define the pathways travelled by energy and matter. An explanation of the mathematical definition of feedback in this context can be found in Appendix 1 (also see Levins 1974). Briefly, feedbacks can cause the instability of a QM in two ways (Dambacher et al. 2003b): 1) positive feedbacks cause the self-amplification of a perturbation, causing the system to drift away from the equilibrium in the direction of the displacement (Levins 1974); and 2) short feedbacks (e.g. between 2-3 variables) are weak or ambiguous, whereas higher order pathways connecting many components of the system are strong, causing general instability. Stability analysis (Dambacher et al. 2003b) has the aims to identify these sources of instability in the system (see Appendix 1 for discussion of the stability criteria).

After evaluating model stability, the effects of a press perturbation on the system can be explored. The press perturbation of a variable in qualitative modelling is defined as a prolonged change (increase or decline) in a rate of birth, death, or migration of said variable (Puccia and Levins 1985). This change can have flow-on effects on the rest of the system, due to the way the perturbed variable is linked to other system components. One way to explore how a perturbation propagates in the system is the study of the adjoint matrix $\operatorname{adj}(\mathbf{A})$ (see Appendix 1). Mathematically, given the community matrix $\mathbf{A}, \operatorname{adj}(\mathbf{A})$ is defined as the transpose of the 
matrix of the cofactors (Searle 1966). Each element $a_{i, j}$ of the adjoint matrix specifies the effect that a positive input in variable $j$ has on variable $i$. That is, the adjoint matrix details the effect that an increase in one variable has on all the other system variables. From an ecological standpoint, each element $a_{i, j}$ of the adjoint matrix is given by the net number of feedbacks (i.e. pathways of ecological interactions) that link the perturbed variable $j$ to any other system variable $i$. Because such feedbacks can be complex, as they define complex ecological interactions between many system components, the predictions of a qualitative model can be prone to ambiguity, or sign indeterminacy. Therefore, it is important to quantify the uncertainty of the predictions of the adjoint matrix (Dambacher et al. 2003a). For this purpose, a matrix of the weighted predictions $(\mathbf{W})$ can be derived from the community matrix $\mathbf{A}$. Elements $\mathbf{w}_{i, j}$ in W detail the likelihood that the responses of the system to perturbation detailed in the adjoint matrix are accurate (see Appendix 1 for details). A prediction weight of $\mathrm{w}=0$ means complete ambiguity of prediction, and $w=1$ means complete certainty (Dambacher et al. 2003a). With numerical simulations, Dambacher et al. (2003a) showed that a value of $w \geq 0.5$ corresponds to accuracy of the predictions of $>90 \%$. The quantification of the uncertainty in the predictions from the adjoint matrix is a fundamental step of the QM approach, because ambiguous predictions are not helpful in elucidating system response to perturbation.

\subsubsection{A qualitative model of a coral reef: model assumptions}

A qualitative model (hereafter referred to as QM1) of a coral reef was built (Fig. 2.2a). QM1 captured some of the main ecological components and interactions of a coral reef, with a focus on corals, sponges, and non-calcifying macroalgae as main benthic competitors. Coral reef benthic communities are complex and diverse, and other sessile organisms (including calcifying algae, ascidians, and soft corals) have important functional roles in coral reef ecosystems. However, for mathematical tractability to be maintained, model variables and connections in a QM should represent only the organisms and processes that are central to the modelled dynamics (Dambacher et al. 2002). In the present study, corals, sponges and macroalgae were chosen to represent the benthos because of their important ecological roles, because they are all impacted by disturbances related to environmental change, and because the responses of sponges and macroalgae to a change in coral abundance was of central interest in this investigation. Instead of modelling spatial competition as direct negative links between the 3 benthic groups (see coral-algae relationships in Harborne et al. 2017), free substrate was included as explicit model variable. This allowed for the modelling of direct interactions between the benthic groups in addition to spatial competition, namely: sponge-mediated 
bioerosion (Schönberg et al. 2017), production of harmful allelochemical (Rossi et al. 2015) and smothering (Loh et al. 2015) as negative link from sponges to corals; and macroalgaemediated smothering as negative link from macroalgae to corals (see Hughes 1994). Lower trophic levels were represented as one pool of picoplankton sustaining the heterotrophic feeding of sponges (Ribes et al. 1999, Hadas et al. 2009), and a simple phytoplanktonzooplankton chain for the heterotrophic feeding of corals. QM1 captured the feeding of sponges on dissolved organic carbon (DOC, de Goeij et al. 2008, Hoer et al. 2018), and the transfer of that energy back to detritus via the sponge loop (de Goeij et al. 2013, Rix et al. 2018) and to spongivorous fishes via sponge predation (McMurray et al. 2018). The sponge loop hypothesis is based on macroalgae and corals contributing to the DOC pool by producing cell exudates (de Goeij et al. 2013), but QM1 did not explicitly model links between producers and DOC to maintain tractability. QM1 included herbivorous fishes grazing on macroalgae, and piscivorous fishes feeding on spongivores and grazers. While in reality all components of the system produce detritus, only links to detritus from sponges (via the sponge loop) and from piscivorous fishes were modelled explicitly. Because piscivorous fishes are at the top of the food web in QM1, their one-way contribution to detritus was proportional to the flow of matter through the system, and thus represented an approximation of each variable's input into the detrital pool. This approximation was necessary for the number of links in the system to remain tractable (Dambacher et al. 2002). In QM1, corals contributed to habitat complexity, which in turn had a positive effect on intermediate trophic levels by providing refuge space from predators (reviewed in Graham and Nash 2013). All variables in QM1 were self-dampened, that is, had a negative self-effect. Self-dampening represents limitations to the growth of a variable that are not explicitly modelled, for example light and nutrients limiting primary producers (sensu Puccia and Levins 1985), or density-dependence limiting population growth (Dambacher et al. 2003a). 


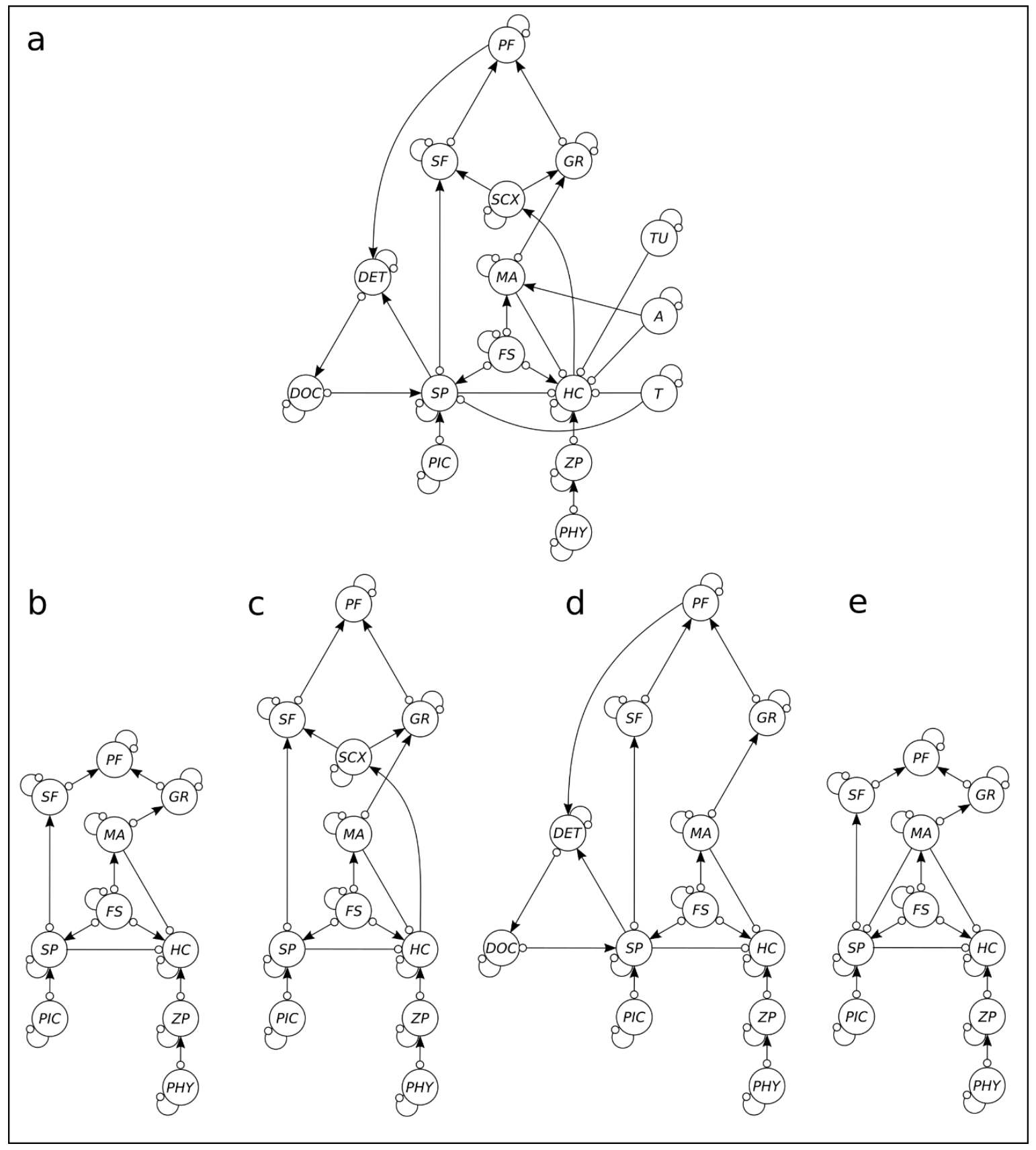

Figure 2.2. Qualitative models of a coral reef. (a) Complete - QM1; (b) simplified model QM2; (c) structural complexity model - QM3; (d) sponge loop model - QM4; (e) macroalgal competitive superiority model - QM5. In order from top to bottom: piscivorous fishes (PF), spongivorous fishes (SF), grazers (GR), structural complexity (SCX), turbidity (TU), detritus (DET), macroalgae (MA), acidity (A), free space (FS), dissolved organic carbon (DOC), sponges (SP), hard corals (HC), temperature (T), picoplankton (PIC), zooplankton (ZP), phytoplankton (PHY). The ends of the arrows indicate the effect of the source variable on the target variable, with $(\rightarrow)$ denoting a positive effect, $(-\circ)$ a negative effect, and $(-)$ no effect. 
QM1 captured 3 physical variables: seawater temperature, acidity, and turbidity. Increased temperature was assumed to have a negative effect on corals (e.g. Hughes 2017) and on sponges (e.g. Bennett et al. 2017, Ramsby et al. 2018); increased acidity was assumed to have a negative effect on corals (Hoegh-Guldberg et al. 2007, Anthony et al. 2008), and a positive effect on some non-calcifying algae, caused by the co-occurring increase in dissolved $\mathrm{CO}_{2}$ and $\mathrm{HCO}_{3}{ }^{-}$ that algae can use for photosynthesis (e.g. Hall-Spencer et al. 2008, Connell and Russell 2010, Cornwall et al. 2017); and increased turbidity was assumed to have a negative effect on corals (Bessell-Browne et al. 2017). In reality, these 3 variables (and many other environmental drivers, including diseases, see Webster 2007) affect all components of reef ecosystems in complex ways (Harborne et al. 2017). However, the focus of this investigation was to determine how the effects of a perturbation acting on the benthos propagate across the ecosystem. Because qualitative modelling does not capture the strength of the effects of one variable on another, a parsimonious model that includes only the perturbations of concern and their strongest (or better known) effects in the investigated context is desirable (Dambacher et al. 2015). Therefore, the model deliberately captured only the main effects of these variables on the main benthic organisms. QM1 was evaluated for stability according to the criteria proposed by Dambacher et al. (2003b).

\subsubsection{Model application: cascading effects of shifts in benthic dominance}

\subsubsection{Ecosystem-level effects of environmental change}

To simulate a scenario of climate change and decrease in water quality, QM1 was used to explore the effects on the ecosystem of a simultaneous increase in temperature, acidity and turbidity (positive input on the 3 variables). Sign determinacy of the predictions from QM1 was evaluated by examining the matrix of weighted predictions W. Prediction weights of $w \geq$ 0.5 were considered unambiguous (Dambacher et al. 2003a). Because of the large number of variables and links in QM1, predictions from it were prone to a high degree of sign indeterminacy (Dambacher et al. 2002, 2003a). To better explore the effects of a perturbationdriven coral decline, and to alleviate the sign indeterminacy of the QM1 (Dambacher et al. 2002), 4 additional models were constructed.

\subsubsection{Ecosystem-level effects of changes in benthic dominance: simplified model}

First, a simplified model (QM2) that did not include the environmental variables, structural complexity, or the sponge loop was created (Fig. 2.2b). Corals had an unambiguous negative response to an increase in temperature, acidity, and turbidity in QM1. Therefore, the removal 
of the 3 physical variables and the implicit modelling of the climate-driven perturbation as coral decline (perturbation 1) was considered acceptable. Moreover, if the decline in corals caused an unambiguous increase in sponge abundance in QM2, the model was used to apply a second perturbation, in the form of an increase in absolute sponge abundance (perturbation 2). Applying these two perturbations directly to corals and sponges was useful to compare ecosystem-level effects between scenarios of relative increase of sponge abundance (due to a decline of corals) and of absolute increase of sponge abundance.

\subsubsection{Roles of structural complexity, sponge loop, and competition in regime shifts}

QM2 was then expanded into 2 additional models of intermediate complexity that had the goal to test the importance of reef structural complexity (QM3, Fig. 2.2c) and of the sponge loop (QM4, Fig. 2.2d) in climate-driven transitioning reefs. For both models, ecosystem responses to the same two perturbations applied to QM2 were explored (i.e. response to a decline in corals and to an increase in sponges).

Finally, QM2 was modified to explore the effects of a decline of corals on the reef ecosystem when assuming general competitive superiority of macroalgae on coral reef sponges (QM5, Fig. 2.2e). Competitive interactions between macroalgae and sponges on coral reefs have not been extensively studied yet (but see Lopez-Victoria et al. 2006 and González-Rivero et al. 2011, 2012, 2016 for examples of competitive superiority of macroalgae). Two perturbations were applied to QM5: a decline of corals; and an increase in macroalgae. The second perturbation in this case was not an increase in sponges, because sponges did not show an unambiguous positive response to the decline of corals in QM5, while macroalgae did.

All models were tested for stability (Dambacher et al. 2003b) and the sign determinacy of their predictions were evaluated (Dambacher et al. 2003a). Signed digraphs were developed with the software PowerPlay Digraph Editor, Version 2.0 (2002). Stability and perturbation analysis were carried out with the mathematical software available at Supplement 1 of Dambacher et al. (2002) in Ecological Archives E083-022-S1 at http://www.esapubs.org/archive/. 


\subsection{Results}

\subsubsection{Ecosystem-level effects of environmental change}

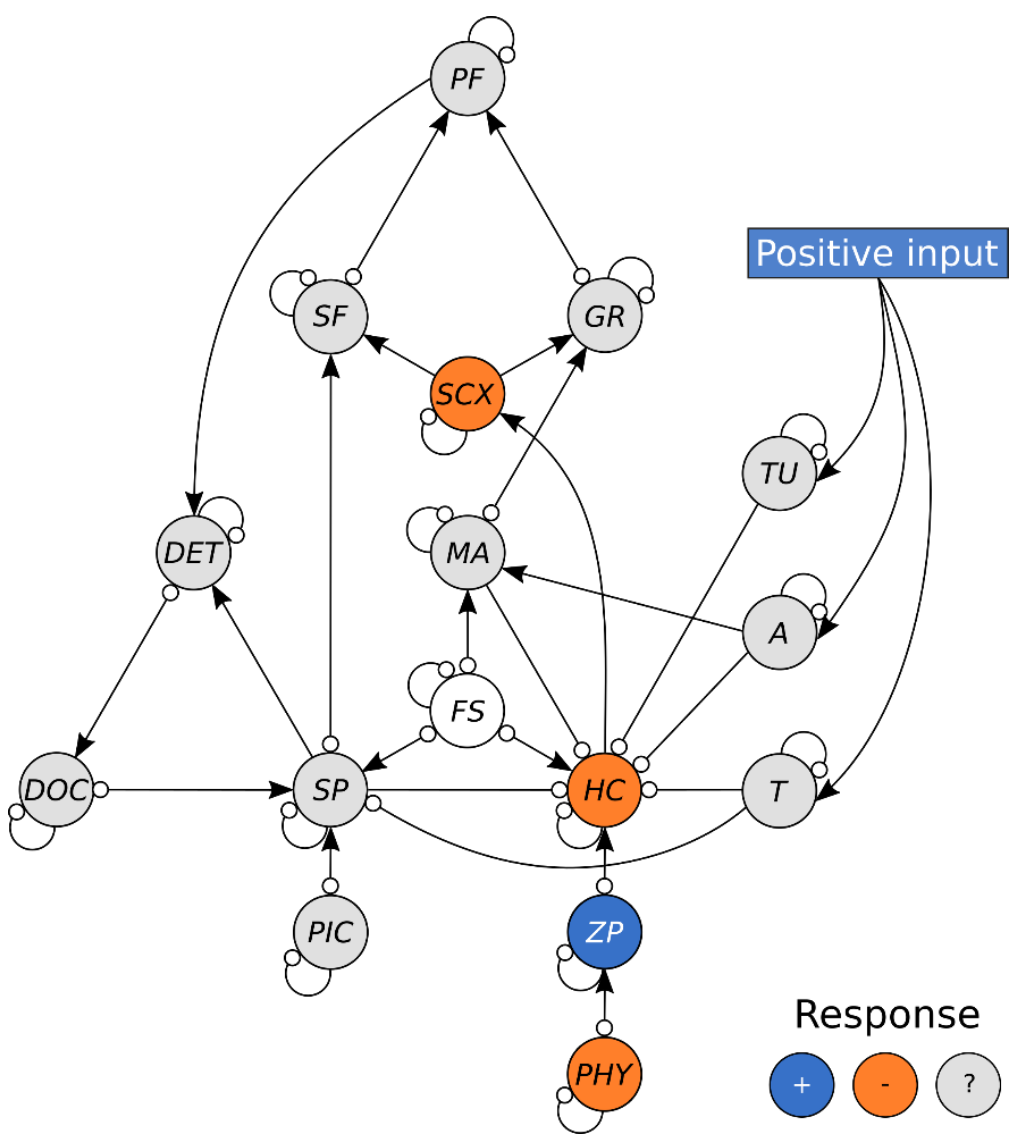

Figure 2.3. Effects of a simultaneous increase in temperature, acidity, and turbidity as potential outcomes of climate change and human impact on coral reefs, with associated responses. In order from top to bottom: piscivorous fishes (PF), spongivorous fishes (SF), grazers (GR), structural complexity (SCX), turbidity (TU), detritus (DET), macroalgae (MA), acidity (A), free space (FS), dissolved organic carbon (DOC), sponges (SP), hard corals (HC), temperature (T), picoplankton (PIC), zooplankton (ZP), phytoplankton (PHY). The ends of the arrows indicate the effect of the source variable on the target variable, with $(\rightarrow)$ denoting a positive effect, (-०) a negative effect, and (-) no effect. Free space was incorporated in the model as a state variable in order to explicitly represent passive occupation of the substrate, alongside specific interactions between benthic groups. However, increases or decreases in free space were not reported as model results, because fluctuations in free space would be difficult to interpret in the context of three benthic groups competing for it. Modified from Bell et al. (2018a), Fig. 1. 
An increase in temperature, acidity and turbidity in QM1 caused the unambiguous negative response of corals and habitat complexity ( $w=0.53$ for both variables), but most model predictions had high sign indeterminacy (Fig. 2.3). The decline in corals had the effect of releasing zooplankton from coral grazing, with the consequent depletion of phytoplankton $(w$ $=0.53$ for both plankton pools). While free space was included as explicit variable in all models, predicted outcomes for this variable were disregarded because they would be of difficult interpretation, due to the three benthic groups competing for free substrate. Unambiguous model predictions and sign determinacy are reported in Table 2.1.

\subsubsection{Ecosystem-level effects of changes in benthic dominance}

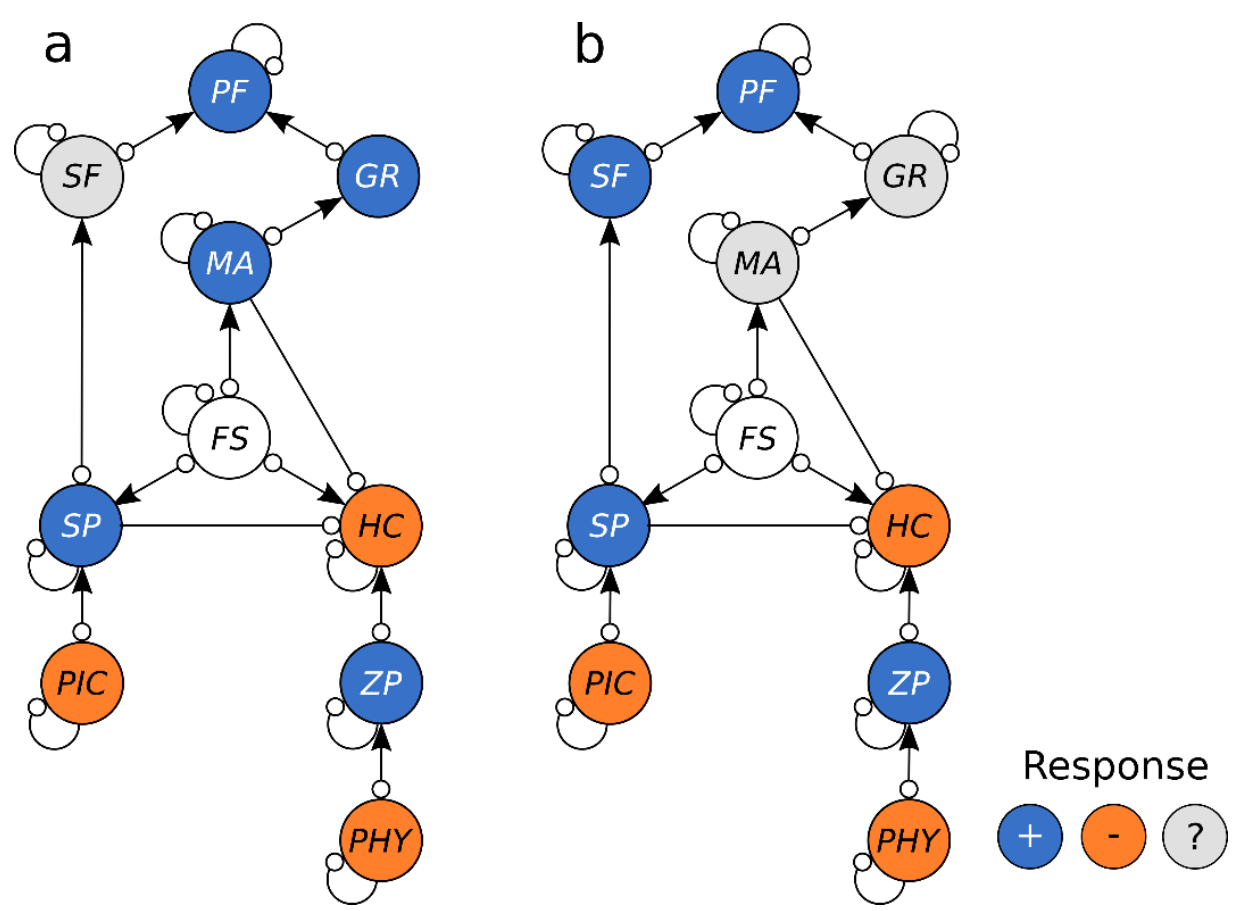

Figure 2.4. Effects of changes in benthic dominance predicted by QM2. (a) Perturbation provides a negative input on corals. (b) Perturbation provides a positive input on sponges. In order from top to bottom: piscivorous fish (PF), spongivorous fish (SF), grazers (GR), macroalgae (MA), free space (FS), sponges (SP), hard corals (HC), picoplankton (PIC), zooplankton (ZP), phytoplankton (PHY). The ends of the arrows indicate the effect of the source variable on the target variable, with $(\rightarrow)$ denoting a positive effect, $(-\circ)$ a negative effect, and (-) no effect. Modified from Bell et al. (2018a), Fig. 2.

Sign determinacy increased considerably for QM2, due to the lower number of variables and links in the system. A perturbation causing a decline in coral abundance resulted in unambiguous positive responses of sponges and algae ( $w=1$ for both variables). The positive 
response of sponges caused in turn the depletion of the picoplankton pool, whereas the positive response of algae propagated to upper trophic levels, causing an increase in grazers and piscivorous fishes ( $w=0.67$ and $w=1$, respectively, Fig. 2.4a; but see predictions from QM3 below). A perturbation-induced increase in sponge abundance caused a negative response of corals, due to the direct negative effects of sponge-mediated bioerosion and smothering of corals and to the pre-emption of free substrate $(w=0.67)$, while the effect of increased sponge abundance on macroalgae and their grazers was ambiguous (Fig. 2.4b). Spongivorous fishes were predicted to increase in this scenario as a consequence of the increased sponge biomass $(w=0.60)$.

\subsubsection{Roles of structural complexity, sponge loop, and competition in regime shifts}

When reef structural complexity was reintroduced as a model variable with a positive effect on grazers and spongivores in QM3, sponges and algae responded positively to a decline in corals $(w=0.50, w=0.55$, respectively, Fig. 2.5a), similarly as in QM2. A decline in corals caused the negative response of reef structural complexity $(w=1)$, which had negative flowon effects on grazers and spongivores. However, the increase in food availability for grazers and spongivores in QM3 contrasted the negative impact of reduced structural complexity, resulting in ambiguous predictions for spongivores and grazers. Piscivorous fishes responded negatively to the decline in corals and associated structural complexity $(w=0.55)$. A perturbation causing an increase in sponge abundance resulted in a negative response of corals and a corresponding decline in structural complexity ( $w=0.67$ for both variables, Fig. 2.5b). However, most other predictions were ambiguous, including the response of sponges.

Predictions from QM4 of the effects of a decline in corals were similar to QM2 (Fig. 2.5c). The negative perturbation acting on corals propagated with positive effects on sponges $(w=1)$ and macroalgae $(w=0.68)$, ambiguous responses on grazers and spongivores, and an overall positive effect on piscivorous fishes $(w=0.83)$. The positive response of the top trophic level in QM4 was due to the enhanced trophic pathways of sponges and algae. The model also predicted an increase in detritus in the system $(w=1)$. An absolute increase in sponge abundance had similar responses (Fig. 2.5d), with the exception of an ambiguous response of 

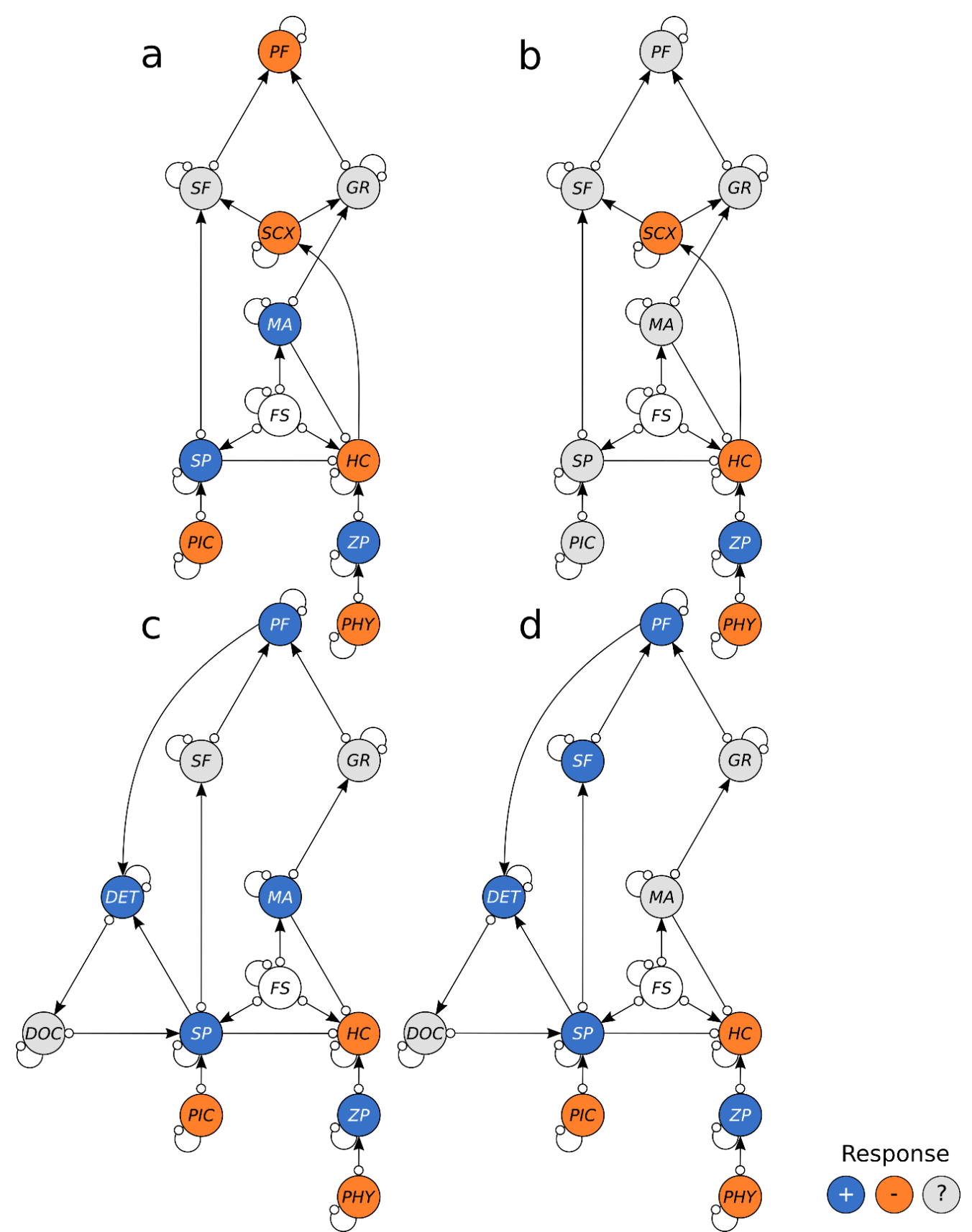

Figure 2.5. Effects of changes in benthic dominance predicted by QM3 (a-b) and QM4 (c-d). $(a, c)$ Response of each system to negative press perturbation acting on corals; (b, d) response of each system to positive press perturbation acting on sponges. In order from top to bottom: piscivorous fish (PF), spongivorous fish (SF), grazers (GR), structural complexity (SCX), macroalgae (MA), free space (FS), sponges (SP), hard corals (HC), picoplankton (PIC), zooplankton (ZP), phytoplankton (PHY), detritus (DET), dissolved organic carbon (DOC). The ends of the arrows indicate the effect of the source variable on the target variable, with $(\rightarrow)$ denoting a positive effect, $(-\circ)$ a negative effect, and (-) no effect. Modified from Bell et al. (2018a), Fig. 3.

macroalgae, and positive response of spongivorous fishes $(w=0.60)$ linked to the increase in 
sponges.

When QM2 was modified to assume competitive superiority of macroalgae over sponges (QM5), a negative perturbation causing a decline of corals caused unambiguous positive responses from macroalgae, grazers and piscivorous fishes $(w=1, w=0.71$, and $w=0.67$, respectively), and an ambiguous response of sponges and their associated source of food and predators (Fig. 2.6a). A positive perturbation acting on macroalgae caused largely ambiguous responses throughout the system, except for a positive response of grazers and a negative one of spongivorous fish ( $w=0.73, w=0.66$, respectively, Fig. 2.6b).

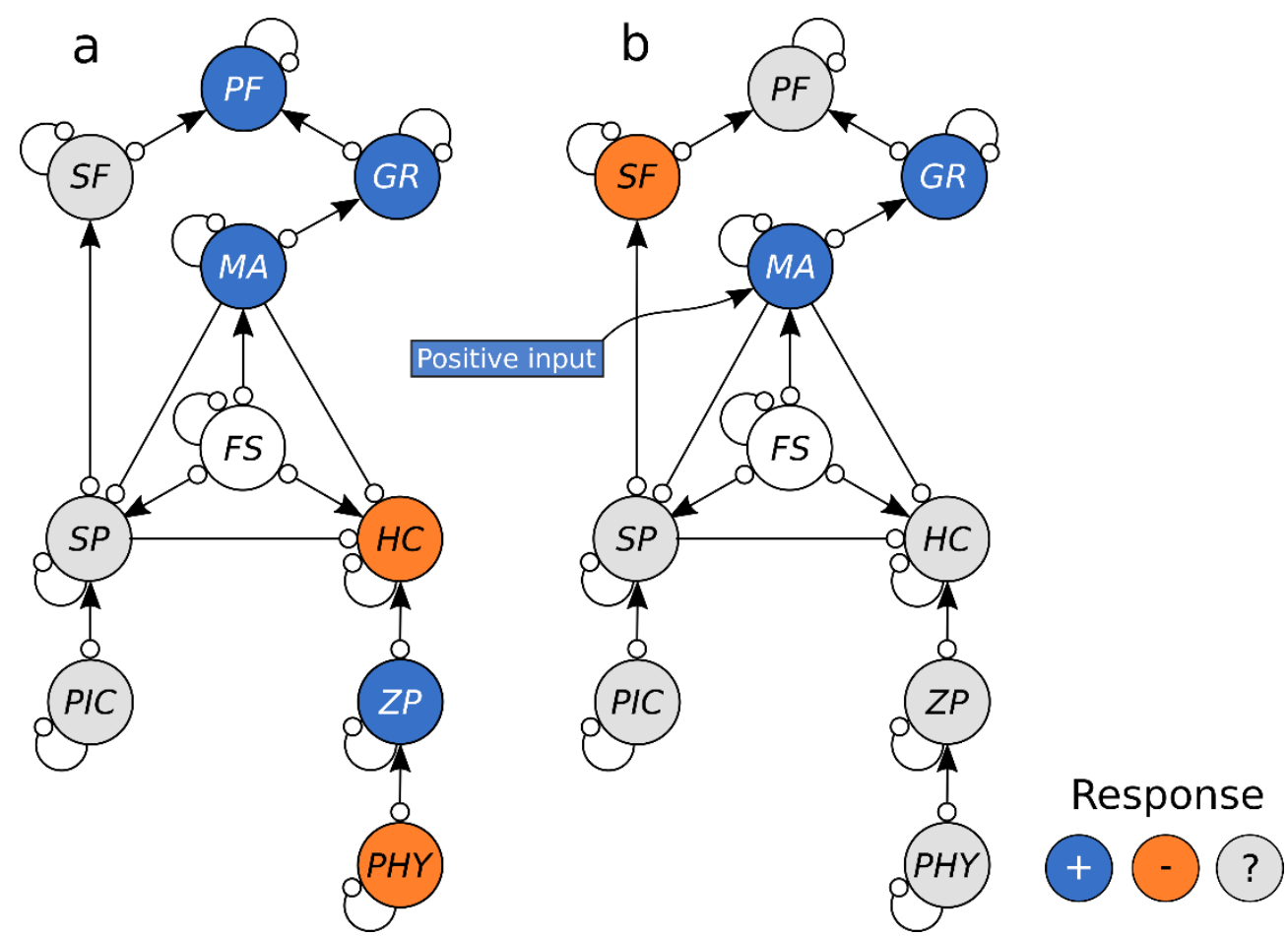

Figure 2.6. Effects of changes in benthic dominance predicted by QM5. (a) Perturbation provides a negative input on corals. (b) Perturbation provides a positive input on algae. In order from top to bottom: piscivorous fish (PF), spongivorous fish (SF), grazers (GR), macroalgae (MA), free space (FS), sponges (SP), hard corals (HC), picoplankton (PIC), zooplankton (ZP), phytoplankton (PHY). The ends of the arrows indicate the effect of the source variable on the target variable, with $(\rightarrow)$ a positive effect, $(-\circ)$ a negative effect, and $(-)$ no effect. Modified from Bell et al. (2018a), Fig. 4. 
Table 2.1. Response of the modelled functional groups to the applied press perturbations (first row). Responses are positive (+), negative (-), or ambiguous (?). For unambiguous responses, the weighted prediction (w) is indicated. W ranges from 0 to 1 , with $w=0$ corresponding to complete ambiguity and $w=1$ to complete certainty. A prediction weight of $w \geq 0.5$ has been found to correspond to $>90 \%$ correct predictions (Dambacher et al. 2003a, see also Appendix 1).

\begin{tabular}{|c|c|c|c|c|c|c|c|c|c|}
\hline \multirow[b]{2}{*}{ Perturbation applied } & \multirow{2}{*}{$\begin{array}{c}\text { Compound model } \\
\uparrow \text { temperature, acidity, } \\
\text { turbidity }\end{array}$} & \multicolumn{2}{|c|}{ Core model } & \multicolumn{2}{|c|}{$\begin{array}{l}\text { Habitat complexity } \\
\text { model }\end{array}$} & \multicolumn{2}{|c|}{ Sponge loop model } & \multicolumn{2}{|c|}{$\begin{array}{c}\text { Macroalgal dominance } \\
\text { model }\end{array}$} \\
\hline & & $\downarrow$ corals & $\begin{array}{c}\uparrow \\
\text { sponges }\end{array}$ & $\downarrow$ corals & $\begin{array}{c}\uparrow \\
\text { sponges }\end{array}$ & $\downarrow$ corals & $\begin{array}{c}\uparrow \\
\text { sponges }\end{array}$ & $\downarrow$ corals & $\uparrow$ macroalgae \\
\hline Sponges & $?$ & $+(1)$ & $+(0.70)$ & $+(0.50)$ & $?$ & $+(1)$ & $+(0.70)$ & $?$ & $?$ \\
\hline Hard corals & $-(0.53)$ & $-(1)$ & $-(0.67)$ & $-(1)$ & $-(0.67)$ & $-(0.70)$ & $-(0.67)$ & $-(0.81)$ & $?$ \\
\hline Macroalgae & $?$ & $+(1)$ & $?$ & $+(0.55)$ & $?$ & $+(0.68)$ & $?$ & $+(1)$ & $+(0.78)$ \\
\hline Spongivorous fish & $?$ & $?$ & $+(0.60)$ & $?$ & $?$ & $?$ & $+(0.60)$ & $?$ & $-(0.66)$ \\
\hline Grazers & $?$ & $+(0.67)$ & $?$ & $?$ & $?$ & $?$ & $?$ & $+(0.71)$ & $+(0.73)$ \\
\hline Piscivorous fish & $?$ & $+(1)$ & $+(0.50)$ & $-(0.55)$ & $?$ & $+(0.83)$ & $+(0.5)$ & $+(0.67)$ & $?$ \\
\hline Picoplankton & $?$ & $-(1)$ & $-(0.70)$ & $-(0.50)$ & $?$ & $-(1)$ & $-(0.7)$ & $?$ & $?$ \\
\hline Zooplankton & $+(0.53)$ & $+(1)$ & $+(0.67)$ & $+(1)$ & $+(0.67)$ & $+(0.71)$ & $+(0.67)$ & $+(0.81)$ & $?$ \\
\hline Phytoplankton & $-(0.53)$ & $-(1)$ & $-(0.67)$ & $-(1)$ & $-(0.67)$ & $-(0.71)$ & $-(0.67)$ & $-(0.81)$ & $?$ \\
\hline Habitat complexity & $-(0.53)$ & & & $-(1)$ & $-(0.67)$ & & & & \\
\hline Detritus & $?$ & & & & & $+(1)$ & $+(0.66)$ & & \\
\hline DOC & $?$ & & & & & $?$ & $?$ & & \\
\hline
\end{tabular}




\subsection{Discussion}

In this chapter I explored the effects of climate-driven perturbations on a coral reef ecosystem using qualitative modelling, with the goal of highlighting the key ecological pathways that will determine how a sponge-dominated reef will function. The specific aims of this chapter were: 1) to obtain qualitative predictions of the effects of an increase in temperature, acidity and turbidity on a coral reef, by using a qualitative model (QM); 2) to use a simplified version of this QM to explore the flow-on effects of a transition towards sponge dominance; and 3) to use three alternative QMs that captured, respectively, reef structural complexity, the sponge loop, and macroalgal competitive superiority over sponges, to evaluate the role of these ecological processes on transitioning reefs. Model predictions showed that projected environmental change will have negative effects on hard corals, and ecosystem-levels effect that will depend on the interplay of many ecological processes. If a shift towards sponge and macroalgal dominance occurs on future reefs, reef secondary production will be enhanced but reef structural complexity will decline, with uncertain effects on reef fishes. Furthermore, an increase in sponge dominance will likely boost sponge-mediated detrital pathways, and sponge populations may be limited by DOC and POC availability on future reefs. This chapter highlighted that, to understand how transitions to sponge dominance may occur and how sponge-dominated reefs may function, some key aspects of sponge ecology must be addressed. These aspects include temporal variability of sponge assemblages, and sponge contribution to reef structural complexity.

\subsubsection{Climate change alterations to a coral reef ecosystem}

QM1 predicted an unambiguous decline in corals and a consequent loss of reef structural complexity, but predictions for most other variables had high sign indeterminacy. This was due to the high number of variables $(n=16)$ and feedbacks in the model. In a $\mathrm{QM}$, the number of feedbacks increases factorially with the number of system variables (Dambacher et al. 2002). This poses a practical limit to the size of the system, whereby even a QM capturing even relatively few variables can yield highly ambiguous predictions due to an intractable number of modelled pathways (Dambacher et al. 2003b). However, the overall low sign determinacy of the predictions from QM1 also highlighted some current gaps in the knowledge of how reef food webs will respond to a long-term decline in corals. These gaps include, for example, the effects of a shift in dominant benthic organisms on reef trophodynamics and three-dimensional structural complexity, and the interactions between sponges and macroalgae after a decrease in coral dominance. Harborne et al. (2017) showed in their review that the overall effects of 
environmental change and anthropogenic pressure on coral reef ecosystems are often uncertain, due to the large number of components and the complex ecological interactions between them. While the uncertainty of model predictions is inevitably tied to the inherent complexity of coral reefs, sign indeterminacy caused by model complexity was mitigated by generating alternative models of lower complexity QM2-5 (see Dambacher et al. 2002).

Qualitative models do not detail the strength of interactions between system variables. Therefore, in this QM, the response of the benthic community to a perturbation-driven decline of corals depended on the sign (positive, negative, or neutral) of the links connecting the coralmacroalgae-sponge sub-system. However, in reality, the response of the benthic community will also depend on the ability of organisms to occupy the free substrate that becomes available after coral mortality. Macroalgae in tropical systems are efficient pioneering colonisers (Arnold and Steneck 2011) that can pre-empt free substrate under low grazing pressure (GonzálezRivero et al. 2016). On the other hand, while sponge assemblages typically develop slowly (Pawlik and McMurray 2020), strong temporal changes in sponge abundance have been observed on some reefs (e.g. Kelmo et al. 2013, Biggerstaff et al. 2017b). In general, temporal variability of sponge assemblages has received little attention so far. To better understand whether transitions towards sponge dominance are likely to occur after coral mortality, it is important to determine the temporal variability of the abundance of sponges, algae and other benthic groups.

Quantitative models should complement qualitative frameworks, when possible. Quantitative models capture ecological processes quantitatively, allowing for more detailed representation of complex ecosystems, and for quantitative predictions. However, quantitative models require a quantitative knowledge of the modelled system. The ecology of sponge-dominated reefs is not well known, largely because there are not many examples of these reef configurations (Pawlik and McMurray 2020). In addition, even when information on the studied system is abundant, qualitative models highlight the ecological feedbacks that are important in the scope of a change in one (or many) system components. Finally, analysing the uncertainty of a qualitative models is a crucial step in detecting the main gaps in the knowledge of the system. This information is needed to guide the development of quantitative frameworks. 


\subsubsection{Ecosystem-level effects of changes in benthic dominance}

Predictions from QM2 showed that a decline in corals had positive effects on sponges and macroalgae, due to the higher availability of free substrate that followed coral mortality. The boost in sponge biomass in this scenario had negative cascading effects on the pool of picoplankton grazed by sponges. Picoplankton is an important component of sponge heterotrophic diet (Reiswig 1971a, 1975, Ribes et al. 2005, Hadas et al. 2009). Because spongemediated carbon fluxes depend on sponge demographics (McMurray et al. 2017), increased sponge abundance on future reefs may require higher concentrations of picoplankton to support sponge heterotrophic feeding, especially on reefs where heterotrophic sponges are the dominant component of sponge assemblages, like the Caribbean (Wilkinson 1987).

QM2 predicted that responses of the fish groups to a decline in corals were different from their responses to an absolute increase in sponge abundance. A decline in corals and the consequent positive response of macroalgae caused an unambiguous positive response in grazers and in piscivorous fishes. Climate change alterations to coral reefs are predicted to strengthen herbivorous trophic links (Harborne et al. 2017), because secondary production is enhanced on macroalgal reefs (see Fulton et al. 2019), with a positive effect on herbivorous fishes (Gilmour et al. 2013) and positive flow-on effects on consumers. Importantly, the QMs developed for this Chapter did not capture fisheries. The overfishing of herbivorous fishes on transitioning coral reefs removes top-down controls on algae and therefore facilitates a shift towards algal-dominated states (Mumby et al. 2007). The predicted positive response of grazers to shifts towards macroalgal states will be conditional on their exploitation (and on loss of structural complexity - see below).

In QM2, an absolute increase in sponge abundance caused a positive response of spongivorous fishes, but it had undetermined effects on macroalgae and the associated grazers. Such ambiguous responses of macroalgae to increased sponge dominance was a result of the combination of a sponge-mediated decline of corals (beneficial to algae) and the loss of free substrate (detrimental to algae). While macroalgae are efficient competitors of bioeroding sponges in the Caribbean (González-Rivero et al. 2012, 2016), competitive interactions between coral reef sponges and algae have not been extensively studied, and the ambiguity of the model predictions reflects the uncertainty around the relationships between these two benthic groups (see QM5 below). 
The increase in spongivorous fishes on reefs that transitioned towards sponge dominance is conditional upon the ubiquity of significant predator-prey relationship between sponges and spongivores. Trophic interactions between sponges and sponge-eating fishes control sponge assemblages in the Caribbean (reviewed in Pawlik et al. 2018), whereas the role of spongivory in the Indo-Pacific is less clear (Powell et al. 2015). Field studies beyond the scope of this thesis will be needed to understand how increased sponge dominance may affect demographics of sponge predators on transitioning reefs.

\subsubsection{Key ecological processes in shifts towards sponge dominance: alternative models}

\subsubsection{Structural complexity}

In QM3, a negative press perturbation acting on corals caused a decline in reef structural complexity, a decline of piscivorous fishes, and the positive response of macroalgae and sponges. Coral-provided structural complexity has important ecological roles on coral reefs (reviewed in Graham and Nash 2013), and it is associated with the abundance and diversity of reef fishes (Coker et al. 2014), and with reef fishery productivity (Rogers et al. 2014). The decline of scleractinian corals has corresponded to a loss of structural complexity on coral reefs (Alvarez-Filip et al. 2009, Elliott et al. 2018, Magel et al. 2019), causing widespread decline of fish species richness and abundance (e.g. Jones et al. 2004, Graham et al. 2006, Wilson et al. 2006). The projected decline of corals will result in a loss of structural complexity on future reef (Wild et al. 2011), with negative effects for fish communities (Newman et al. 2015) and for reef fisheries (Rogers et al. 2018). The ambiguous response of grazers and spongivores predicted by QM3 was due to a loss of structural complexity (negative) and the discussed boost in productivity tied to the increase in the respective food sources (positive).

When QM3 was perturbed with an absolute increase in sponge abundance, many groups showed ambiguous responses, but hard corals still declined and so did structural complexity. Smothering effects of sponges on corals have been observed in the Caribbean (Loh et al. 2015) and the Indo-Pacific (Rossi et al. 2015), and although sponges may have some beneficial effects on reef accretion by binding coral rubble (Wulff 2006a), an increase in sponge abundance is overall expected to have detrimental effects on corals (Pawlik and McMurray 2020). Moreover, an increase in abundance of bioeroding sponges combined with the projected OA conditions may have negative effects on the accretion-erosion balance of coral reefs (reviewed in Schönberg et al. 2017), although many bioeroding sponge species will likely not tolerate the highest predicted increases in seawater temperature (Fang et al. 2014, Ramsby et al. 2018). 
A key question emerging from QM3 is whether other organisms than corals can also contribute to structural complexity on transitioned reefs. Canopy-forming algae can provide structural complexity on macroalgal reefs, but algal assemblages will also likely shift towards low-complexity states due to increased water temperature (Fulton et al. 2019), with detrimental effects on macroalgal-associated reef fish (Wenger et al. 2018). The role of sponges as providers of structural complexity on coral reefs has not been investigated yet. The high sign indeterminacy of the predictions from QM3 under an absolute increase in sponge abundance highlighted the paucity of information currently available on the ecosystem-level effects of a change towards sponge dominance. Investigating the potential for coral reef sponges to provide structural complexity will be important to gain better insights on how sponge-dominated reefs will likely function.

\subsubsection{The sponge loop}

QM4 predicted an unambiguous increase in detritus. This increase in detritus was caused by sponge production of particulate detritus, which has been reported for many sponge species (Alexander et al. 2014, Rix et al. 2016, 2017, 2018). Notably, DOC showed an ambiguous response to an increase in sponges. Such ambiguous response of DOC was due to two opposite effects: the positive effect from the decomposition of detritus (see Holmer 1996); and a negative effect of the sponge-mediated consumption of DOC. While the reliance of sponges on DOC is species-specific (Hoer et al. 2018), an increase in sponge abundance on coral reefs will likely cause an increase in sponge-mediated carbon flow (McMurray et al. 2017), with higher demand of inorganic carbon to sustain larger sponge populations on transitioned reefs.

Although there is increasing evidence for the existence of the sponge loop on coral reefs (reviewed in Pawlik and McMurray 2020), this pathway has been investigated mostly on Caribbean reef systems. Furthermore, not all sponges produce significant quantities of detritus, and a proposed alternative to the sponge loop is the assimilation of DOC and the production of sponge biomass (McMurray et al. 2018). Finally, corals are large producers of DOC on coral reefs (Wild et al. 2004, Tanaka et al. 2008), and a loss of corals on transitioning reefs may therefore have an indirect curbing effect on the sponge loop by limiting DOC availability. Elucidating the ubiquity and the ecological pervasiveness of the sponge loop with experimental studies on sponge species from different regions will have a critical role in the understanding of sponge-dominated reefs. Assessing the ubiquity of the sponge loop is beyond the scope of this thesis. 


\subsubsection{Competitive superiority of macroalgae over sponges}

When macroalgae were assumed to have a competitive advantage over both corals and sponges (QM5), a decline in corals caused a positive response in macroalgae, which in turn caused an ambiguous response in sponge abundance. This response mirrored the uncertainty around the development of sponge assemblages on reefs that will transition towards algal dominated states. Competitive interactions between sponges and algae on coral reefs have received little attention. Research from the Caribbean showed that proximity with macroalgae is disadvantageous for sponges due to shading and abrasion (Easson et al. 2014), while bioeroding sponges of the genus Cliona are limited by pre-emptive competition of macroalgae (López-Victoria et al. 2006, González-Rivero et al. 2012, 2016). However, relationships between sponges and algae are species-specific (López-Victoria et al. 2006), and the outcome of competitive interactions on transitioned reefs will depend on the species composition of

algal and sponge assemblages. Importantly, the negative effect of an increase in sponge abundance on corals in QM2-4 was mediated by the negative link between sponges and corals. This negative link also captured bioerosion from excavating sponges, and it is therefore important to understand whether macroalgae can reliably curb increases in boring sponge abundance, as this would indirectly remove competitive pressure on corals. Experimental studies beyond the scope of this thesis will be needed to characterise competitive interactions between algae and sponges.

\subsubsection{Emerging key questions and focus of future chapters}

This application of qualitative modelling showed that climate change-induced decline of corals on future coral reefs may result in an increase in sponge and macroalgal abundance, and an enhancement of sponge-driven detrital carbon pathways. Furthermore, the predicted increase in sponge and macroalgal abundance will likely cause a boost in secondary production of herbivores, spongivores and detritivores. However, a decline in structural complexity caused by the loss of corals will have detrimental effects on reef fishes.

By predicting an increase in sponge abundance in response to a perturbation, all QMs used in this analysis assumed that coral reef sponge assemblages can show temporal variability. However, temporal dynamics of tropical sponge assemblages have received limited attention so far. Therefore, it is important to quantify short-term and long-term temporal variability of coral reef sponge assemblages (see Chapter 3), and of other benthic organisms (see Chapter 4). Understanding the temporal development of sponge assemblages (and of the benthic 
community they are a part of,) is crucial to develop and validate quantitative ecosystem models that capture temporal dynamics of coral reef organisms (see Chapter 6).

A key research questions emerged as a result of the analysis of model uncertainty. That is, to understand whether enhanced macroalgae and sponge biomass will support fish productivity on coral-depleted reefs despite the loss of structural complexity, it is important to determine whether sponges can also provide structural complexity (see Chapter 5). In addition, QMs in this chapter highlighted the importance of other aspects of sponge ecology that are of primary importance in the context of a regime shift towards sponge reefs. For example, spongemediated carbon fluxes depending on the consumption of DOC may have a major role in the functioning of sponge-dominated reefs. In particular, the sponge loop pathway may support higher trophic levels by reintroducing carbon in the system in the form of detritus consumed by detritivores. Identifying these key aspects of sponge ecology guided the incorporation of sponges in the Atlantis ecosystem model (Chapter 6). 


\section{Chapter 3. Temporal variability of sponge abundance and biodiversity of an Indonesian sponge assemblage}

This Chapter is published as: Rovellini A., Dunn, M. R., Fulton, E. A., Webster, N. S., Smith, D. J., Jompa, J., Haris, A., Berman, J., and Bell, J. J. (2019) Decadal variability in sponge abundance and biodiversity on an Indo-Pacific coral reef. Marine Ecology Progress Series, 620:63-76. https://doi.org/10.3354/meps12968. Reprinted with permission. Inter-Research is the copyright holder for this article. 


\begin{abstract}
Natural communities can undergo large temporal changes in abundance and species composition that may be difficult to detect without long-term ecological monitoring. Characterizing temporal variability in coral reef fauna is critical for predicting how reef ecosystems will be impacted by environmental change. Sponges are an ecologically important component of coral reefs, yet descriptions of temporal dynamics of multi-species sponge assemblages are scarce. I studied temporal changes in abundance and biodiversity of an Indonesian coral reef sponge assemblage over 13 years (2005-2017). Mean sponge abundance (sponge patches $\mathrm{m}^{-2} \pm \mathrm{SE}$ ) initially increased from $124.06 \pm 8.46$ to $183.73 \pm 12.12$ in 2005 $2007(P<0.001)$, declined to $66.53 \pm 10.62$ in 2007-2014 $(P<0.001)$, and increased to 105.87 \pm 15.42 in 2014-2017 $(P<0.001)$. These patterns in sponge abundance did not depend on water temperature. Overall, 141 sponge taxa were recorded. However, species composition showed strong temporal patterns, driven by a few abundant taxa (e.g. Protosuberites sp., Sycon sp., and Pericharax sp., respectively accounting on average for $\sim 25 \%, 20 \%$ and $5 \%$ of total sponge abundance). Species richness increased with sponge abundance $(P<0.001)$, whereas evenness decreased due to dominance of some taxa in years of high sponge abundance $(P=0.002)$. This Chapter highlights that the abundance and biodiversity of Indo-Pacific sponge assemblages undergo dramatic temporal changes driven by species-specific population variability. This variability has important implications for designing monitoring programs, for interpreting experimental studies, and for understanding long-term responses of coral reefs to perturbations.
\end{abstract}

\title{
3.1. Introduction
}

Demographic patterns and species composition of natural communities can display strong temporal variation. Interannual fluctuations of the abundance of natural populations have been well documented for many organisms; for example, plants (Gonzalez-Andujar et al. 2006), insects (Turchin et al. 1991), marine benthic infaunal communities (Frid et al. 2009), and coral reef organisms (Adjeroud et al. 2002, Shenkar et al. 2008). Within communities and multispecies assemblages, life-history traits of a species can influence its responses to environmental drivers, and this can result in species-specific population dynamics. As a consequence, the species composition and biological diversity of the assemblage can also be temporally variable (e.g. Lamy et al. 2016, Mokany et al. 2015). 
Understanding the temporal dynamics of functional components of coral reef communities is challenging because of the complexity of interactions and the range of factors that drive them. Sponges are an important component of coral reefs, where they perform a variety of functional roles (see Chapter 1). For example, sponges remove particles and organic matter from the water column through filtration (de Goeij et al. 2008), and make them available to higher trophic levels either as sponge detritus eaten by detritivores (Rix et al. 2018) or as sponge biomass eaten by sponge predators (McMurray et al. 2018, Pawlik et al. 2018). Sponges also influence the accretion-erosion balance of reefs, negatively by chemical-mediated bioerosion (Schönberg et al. 2017) and positively by rubble binding (Wulff 1984). Sponges also play an important role in silicon cycling (Maldonado et al. 2005), and provide biogenic habitat for associated microbial and macrofaunal communities (Wulff 2006a, Taylor et al. 2007). In the context of environmental change and reef degradation, sponges are receiving increasing attention due to their potential to become more abundant, and even dominant, on coral reefs with the decline of corals (Norström et al. 2009, Bell et al. 2013, Stubler et al. 2015, Bennett et al. 2017, Bennett et al. 2018). An absolute increase in sponge abundance on coral reefs has already been observed in the Caribbean (Diaz and Rutzler 2001, McMurray et al. 2010), and at certain sites in the Indo-Pacific (Powell et al. 2010, Powell et al. 2014) and tropical Pacific (Schils 2012, Knapp et al. 2013, Knapp et al. 2016). However, studies on temporal dynamics of tropical sponge assemblages are rare; most long-term surveys have been performed in the Caribbean (Butler et al. 1995, Wulff 2006b, Wulff 2013, Wulff 2017, Stevely et al. 2011), although recent reports have also emerged from the Central Pacific (Knapp et al. 2013, Knapp et al. 2016) and Great Barrier Reef (Ramsby et al. 2017).

The Indo-Pacific is an important biodiversity hotspot for marine taxa, including fishes and corals (Roberts et al. 2002). Sponge assemblages in the Indo-Pacific are extremely complex and poorly understood (Bell et al. 2015a, van Soest et al. 2012), particularly with respect to variation in sponge abundance and diversity. Despite the paucity of information on sponge ecology in the Indo-Pacific, sponges from this region fill all the important ecological roles mentioned above: bioerosion (Marlow et al. 2018a), silicon cycling (Bertolino et al. 2017), competitors for space (de Voogd et al. 2004, Rossi et al. 2015), food sources for grazers (Powell et al. 2015), and hosts for bacterial communities (Cleary et al. 2019). Nevertheless, while studies have described the community composition of sponge assemblages at some locations in Indonesia (Cleary and de Voogd 2007, de Voogd and Cleary 2008, de Voogd et al. 2009, Calcinai et al. 2017), and short-term temporal changes in mono-specific sponge populations 
(Biggerstaff et al. 2017b), no information exists on temporal dynamics of entire sponge assemblages. These knowledge gaps severely restrict our understanding of benthic dynamics on potentially threatened reefs. Understanding temporal patterns in abundance and the taxonomic composition of sponge assemblages is important for distinguishing between changes that are a result of natural population variability and those that may be a result of anthropogenic disturbance.

Of the many environmental drivers that can influence the temporal dynamics of a community (see Chapter 1), changes in sea water temperature have variable effects on sponge populations. Widespread sponge mortality has been documented after exposure to increased water temperature in temperate and tropical systems (reviewed in Bell et al. 2017). However, some sponge assemblages are resistant to acute warming associated with El Niño Southern Oscillations (Kelmo et al. 2013), and sponges have been proposed as potential winners on warmer coral reefs under near-future climate scenarios (reviewed in Bell et al. 2018b). Warming trends over the past few decades have been reported for the Indo-Pacific (Heron et al. 2016), and bleaching events in this region have been documented for corals (Bruno and Selig 2007, Wilson et al. 2012) and the bioeroding sponge Cliona aff. viridis (Marlow et al. 2018b). However, the effects of temporal changes in seawater temperature on sponge assemblages in this region remain unexplored.

Long term monitoring is important to understand the effects of perturbations on communities. In this Chapter, I quantified long-term temporal variation of sponge abundance (measured as the number of sponge patches, where a sponge patch is one delimited growth forms of a single sponge taxon), diversity and assemblage composition on an Indonesian coral reef between 2005 and 2017 in order to: (1) characterise the temporal dynamics of the sponge assemblage; (2) investigate the effect of sea water temperature on sponge assemblage population dynamics; (3) investigate whether fluctuations in sponge abundance correspond to changes in the biodiversity of the assemblage; and (4) perform an analysis of the assemblage composition to identify which taxa contribute most to the observed fluctuations in sponge abundance. 


\subsection{Materials and Methods}

\subsubsection{Site description and sampling protocol}

The study location (known locally as Buoy $3,05^{\circ} 28.34 \mathrm{~S}, 123^{\circ} 45.43 \mathrm{E}$ ) is a vertical reef wall off the South-West coast of Hoga Island in the Wakatobi Marine Park (SE Sulawesi, Indonesia, Fig. 3.1). At this location, three sites (A, B and C) approximately $100 \mathrm{~m}$ apart from one another were established in 2005 at a depth of 10-12 m. Shallow water marine sponges generally have limited dispersal capabilities (Maldonado 2006), with dispersal distances in the order of $<25 \mathrm{~m}$ for small encrusting species that produce philopatric larvae (Blanquer et al. 2009) and of 60$140 \mathrm{~m}$ for larger species like Xestospongia spp. in the Indo-Pacific (Bell et al. 2014). Therefore, the distance between sites was considered sufficient to minimise between-site recruitment for most of the surveyed sponge taxa. Due to the configuration of the reef, sites A and B were established on a $90^{\circ}$ wall, whereas site $\mathrm{C}$ was inclined at approx. $80^{\circ}$. At each site, 5 permanent $1 \mathrm{~m}^{2}$ fixed replicate quadrats were established on the vertical reef wall along an approximately $10 \mathrm{~m}$ long reef tract. Permanent quadrats were chosen as the most appropriate method to monitor temporal dynamics of the same sponge assemblage in the same reef area (see Austin 1981) while ensuring consistency between reef surfaces that were being sampled through time (e.g. to standardise for shading, orientation, surface angle and fractal surfaces). The quadrats were positioned haphazardly at each site because if permanent plots are placed by judgement this may give a biased picture of the population (Hill et al. 2006). After establishing the quadrats, initial surveys were conducted to identify the dominant sponge species inhabiting the area. Tissue samples were collected from approximately fifty sponges in the first year to identify the dominant sponge taxa at the highest resolution possible. Taxon identification, where possible, of these sponges was based on external and internal (spicule and structure) morphological features, and carried out with the aid of published resources (e.g. van Soest 1989, Hooper and van Soest 2002). Reference information for in situ identification of the sponges in subsequent years was maintained, with additional sampling performed any time a new taxon was recorded in the quadrats (although material was only able to be exported in the first year for full taxonomic work). While the majority of taxa were not formally identified, the same taxa could be consistently identified through time because the same observer collected all data, allowing overall assemblage dynamics to be investigated. 


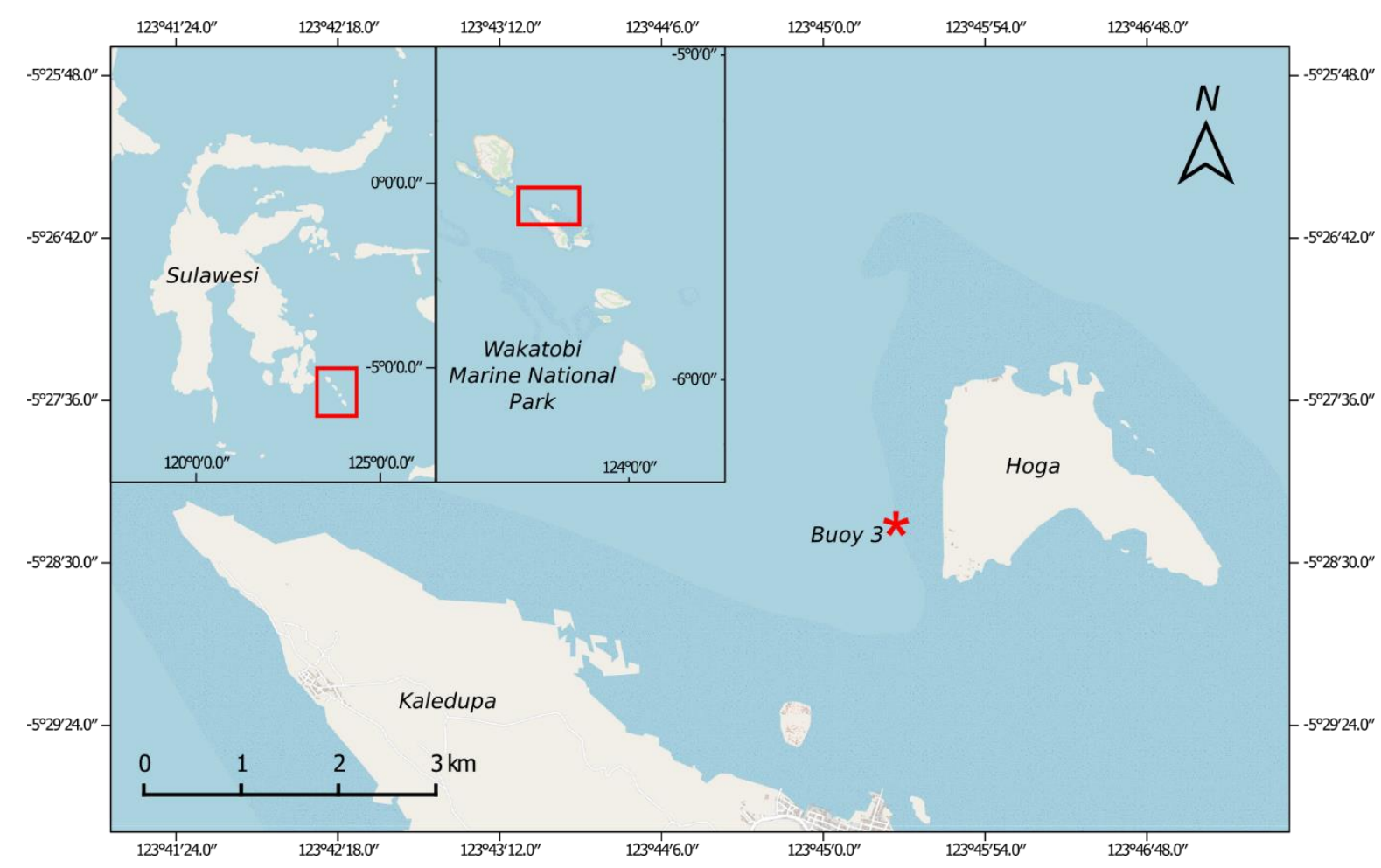

Figure 3.1. Map of Hoga Island, the Wakatobi National Marine Park, and Sulawesi. The star indicates the location of the study site. Map data: (C) OpenStreetMap contributors, available from https://www.openstreetmap.org. Map created in QGIS (QGIS Development Team 2018).

While there are several ways that sponge abundance can be characterised (Bell et al. 2017), here total sponge abundance based on number of patches was selected, where one sponge patch corresponds to a delimited growth form of a single sponge taxon. A sponge patch corresponds to one individual sponge growth form, irrespective of its size, as delimited by its contours. The term 'patch' is used instead of the term 'individual' because many sponges are capable of asexual reproduction and fragmentation. Therefore, the term 'individual' would be ambiguous for a sponge resulting from the fragmentation of another sponge, as the two would be genetically identical. Patches were selected as a measure of sponge abundance for several logistical and practical reasons. The sponge assemblage at this reef is primarily comprised of small encrusting species (ranging from a few $\mathrm{mm}^{2}$ to $\sim 0.25 \mathrm{~m}^{2}$ for the largest sponge, see Fig. A2.1 for scales), found in small patches at high densities (often $>200$ sponges $\mathrm{m}^{-2}$ ). An accurate estimation of the individual volume or biomass of these sponges was therefore impractical due to their morphology (>90\% of sponge patches were encrusting) and dimensions. Furthermore, while photographic methods were trialled to estimate percentage cover, the high diversity and coverage of non-sponge benthic organisms meant a large number of sponges could not be seen 
in the photographs. For these reasons, the number of sponge patches was considered the most appropriate measure to capture sponge abundance and diversity in this study. Sponges were counted in situ on SCUBA between June and July every year from 2005 to 2017, except for 2010 and 2012. Sponges were counted and identified by the same observer. A total of 141 sponge taxa were identified on the reef over the study period. A complete list of the taxonomically identified sponges and pictures of the most common taxa can be found in Appendix 2 (Tab. A2.1, Fig. A2.1).

\subsubsection{Temporal changes in sponge abundance}

Temporal and spatial differences in sponge abundance $(N)$ were explored by fitting linear mixed effect models (LMM, R package nlme, Pinheiro et al. 2018) with normally distributed errors and random intercepts. Despite the limited dispersal range of sponges (Blanquer et al. 2009), between-quadrat recruitment was possible within the same site. Therefore, to account for the quadrat-associated variation in the data, quadrat was used as random factor in all models (Davies and Gray, 2015). Models were built with years from the start of the survey and site as fixed effects, and with quadrat as a random effect to account for between-quadrat variability. To account for repeated measures, a continuous autocorrelation structure of order 1 was fitted to the models. The autocorrelation structure built into the model accounts for dependence of the response variable $N$ at time $t$ on its own value at time $t-1$ (i.e. the previous year), with strength of the correlation declining for measures that are further than one time step away. The most appropriate combination of fixed effects (see Table 3.1 for details of which fixed effects were selected for each model) was selected with the Likelihood Ratio Test (LRT). A variancecovariance matrix accounting for between-quadrat heteroscedasticity was then fitted to the model selected with LRT. Equal variance and normality of the errors were checked for all models with visual inspection of the normalised residuals (Fig. A2.2). Reef-level differences in total sponge abundance between specific time points (e.g. start and end of the survey, and times of maximum and minimum sponge abundance) were then detected with Wilcoxon signed rank tests for paired samples with Bonferroni correction. Differences in total sponge abundance between sites were detected with Mann-Whitney tests with Bonferroni correction. For all tests a significance level of $5 \%$ was used.

\subsubsection{Effects of sea-surface temperature on sponge abundance and growth}

Given total sponge abundance $N$ in each quadrat, for each pair of consecutive years $y$ and $y+1$, the annual percentage population growth rate for sponges in each quadrat was calculated 
as $g=\left[\left(N_{t+1}-N_{t}\right) / N_{t}\right] \times 100$. Sea surface temperature (SST) and SST anomaly (SSTANOM) data for the studied period were obtained from the National Oceanic and Atmospheric Administration's (NOAA) Coral Reef Watch programme (resolution 50 km, Fig. A2.3-2.4, available

at https://www.ospo.noaa.gov/data/cb/TS_vs/vs_ts_Wakatobi_Indonesia.txt, NOAA Coral Reef Watch, 2000). Data from the Wakatobi Virtual Station $\left(5^{\circ} \mathrm{S} 124^{\circ} \mathrm{E}, \sim 59 \mathrm{~km}\right.$ from Buoy 3) was used as it is nearest to the study site. SST and SSTANOM were averaged for the six months (December to May) preceding each sponge survey event. A six-month period was chosen to capture the warmest months preceding each sponge census (Fig. A2.3). LMMs were used to investigate the effect of mean SST and SSTANOM over the six months prior to each sponge sampling event on: 1) sponge abundance $(N)$; and 2) sponge population percentage annual growth $(g)$. Models were built with SST and SSTANOM as fixed effects, quadrat as a random effect, and model structure as described in section 3.2.2. Time series of SST and SSTANOM were decomposed with moving averages to identify trends.

\subsubsection{Species richness, diversity and evenness}

For each quadrat, I calculated species richness, diversity and evenness of the assemblage. Species richness $(S)$ was defined as the number of taxa represented by at least one sponge patch, species diversity was expressed with Shannon's $H^{\prime}$, and evenness with Simpson's evenness index $E_{1 / D}$ (Williams 1964). Species richness alone fails to capture how evenly individuals are distributed among the species in the community, and therefore measuring evenness and diversity is needed (Ma 2005). Simpson's evenness index satisfies the requirement of independence from species richness, and is a commonly used evenness index when the importance of rare species is considered limited (Smith and Wilson 1996). Given the extreme rarity of some of the taxa recorded, this index was deemed the most robust and was selected to complement $H^{\prime}$, which gives the same weight to dominant and rare species. Species richness has been shown to often have a positive relationship with abundance (Bock et al. 2007), because an environment that can provide the energy to support abundant populations can also support a high number of species (Wright 1983). To understand whether temporal changes in sponge abundance would correspond to changes in biodiversity, relationships between $S$ and sponge abundance $(N)$, between $H^{\prime}$ and $N$, and between $E_{1 / D}$ and $N$ were explored with LMMs. Models were built with $N$ and site as a fixed effects, quadrat as a random effect, and a model structure as described in section 3.2.2 (except year was not an effect in these models). 


\subsubsection{Spatio-temporal changes in species composition}

Temporal and spatial variation in the sponge assemblage composition were investigated using a combination of unconstrained and constrained ordination methods (Anderson and Willis 2003). First, the abundances of the 141 sponge taxa per quadrat over the 11 years sampled were $\log$-transformed $[\log (\mathrm{x}+1)]$. Sponge abundance was $\log$-transformed because high abundance of some dominant taxa would have masked the contribution of some of the less common species to sponge assemblage composition. A Bray-Curtis dissimilarity matrix was then calculated for the assemblage. Bray-Curtis dissimilarity describes the resemblance between two samples based on the abundance of the species they share. Bray-Curtis dissimilarity is often used in community ecology as a measure of resemblance between samples that contain many instances of 0-abundance for some species (Legendre and Legendre 2012), which was the case for some less common sponge taxa in the present study. Non-metric Multidimensional Scaling (nMDS) was performed on the matrix. nMDS produced an ordination based on the Bray-Curtis dissimilarity, in order to visualise how similar different quadrats were to each other with respect to their assemblage composition. nMDS was performed using years and site as factors in order to evaluate patterns in the assemblage composition in space and time. The number of $k$-dimensions for the MDS ordination was set to $k=4$. This was derived by iterating the ordination with a growing number of $k$-dimensions, starting from $k=2$, and by determining at which point the stress function fell below 0.2 (considered a cut-off above which the representation of the data on an nMDS plot is no different from random, Clarke 1993). Permutational multivariate analysis of variance, or permutational MANOVA (Anderson 2001) was performed to test for a significant effect of space and time on the assemblage composition. Sites, years, and the interaction between the two were used as fixed factors for the analysis, using 9999 permutations. Finally, a Canonical Analysis of Principal coordinates (CAP) was performed. CAP is a constrained ordination method that explores the variation in multivariate community data based on a distance or dissimilarity metric (Bray-Curtis in this case) by testing the effects of a set of predictors (year and site in this case) on such variation. CAP is useful to disentangle the contribution of the most abundant taxa to the assemblage variation in space and time, and to understand how samples from different sites and years were related in terms of species composition. The analysis of the BrayCurtis matrix was repeated using a square root transformation, and consistency of results was checked to avoid possible biases due to the data transformation. Multivariate analysis was 
performed with the R package vegan (Oksanen et al. 2019). All analyses and plotting were performed in $R$ version 3.4.3 (R Core Team 2019), and the package ggplot2 (Wickham 2009).

\subsection{Results}

\subsection{1. $\quad$ Sponge assemblage dynamics}

Results from LMMs showed that sponge abundance changed over time and between sites $(P$ $=0.015$, Tab. 3.1). Mean sponge abundance (sponge patches $\mathrm{m}^{-2} \pm \mathrm{SE}$ ) at this reef initially increased from $124.06 \pm 8.46$ to $183.73 \pm 12.12(+47 \%, P<0.001)$, then declined to $66.53 \pm$ 10.62 between 2007 and $2014(-63 \%, P<0.001)$, and then increased again to $105.87 \pm 15.42$ between 2014 and $2017(+59 \%, P<0.001)$. No significant change in total sponge abundance was detected between the start and the end of the survey period $(P=0.073)$. This pattern was consistent within and across the three sites. Sponge abundance at site B was generally lower than at sites $\mathrm{A}$ and $\mathrm{C}$ (both $P<0.001)$, and it was similar between sites $\mathrm{A}$ and $\mathrm{C}(P=0.25$, Fig. $3.2)$.

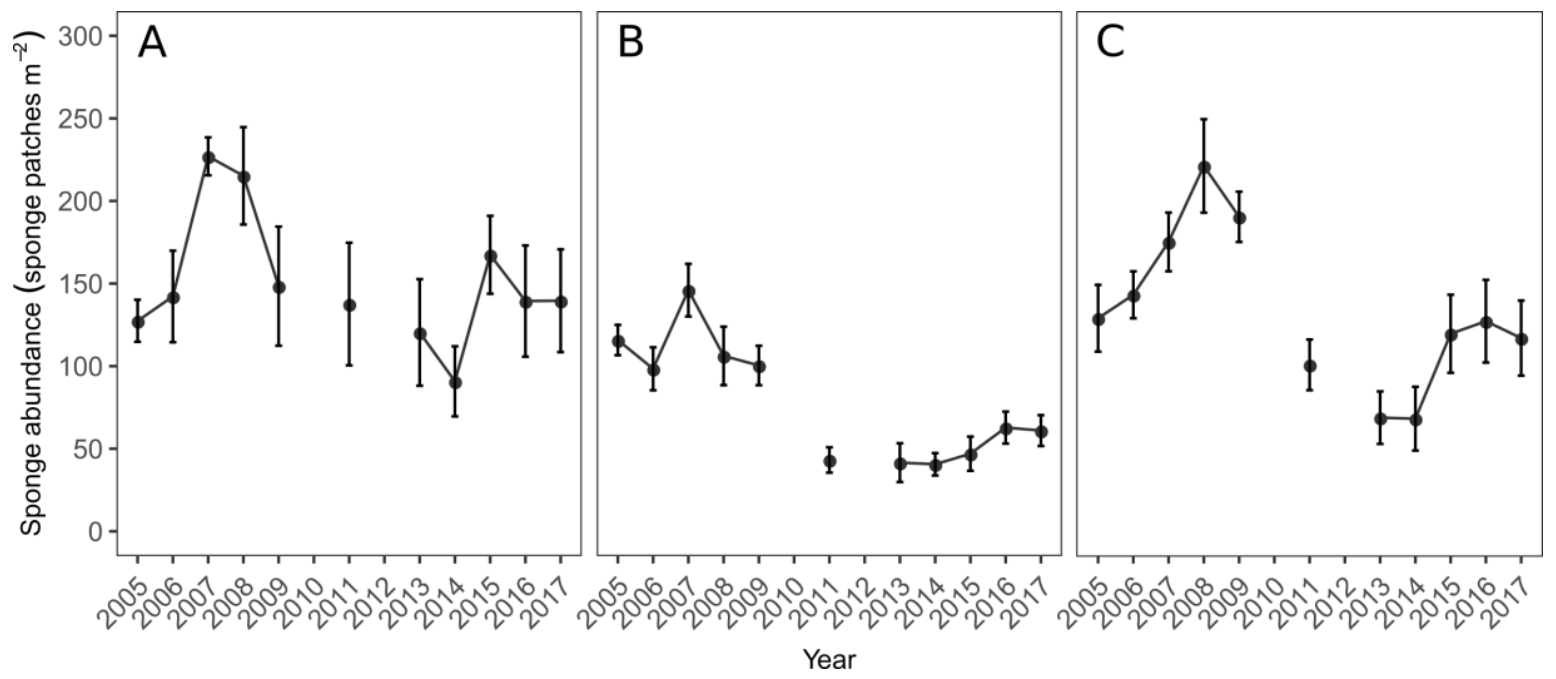

Figure 3.2. Mean total sponge abundance from 2005-2017 at the three sites (letters A-C) at Buoy 3 (Hoga Island, Wakatobi National Marine Park, Indonesia). 
Table 3.1. Coefficients of linear mixed effects models (LMMs). The fixed model term, coefficient estimate, $\mathrm{SE}, \mathrm{DF}, \mathrm{t}$ - and $\mathrm{p}$-value are reported for all dependent variables (sponge abundance $N$, species richness $S$, Shannon's diversity $H^{\prime}$, and Simpson's evenness $E_{l / D}$ ). Significant results $(\mathrm{p}<0.05)$ highlighted in bold.

\begin{tabular}{|c|c|c|c|c|c|c|}
\hline $\begin{array}{l}\text { Dependent } \\
\text { variable }\end{array}$ & Coefficient & Estimate & Standard error & $\begin{array}{l}\text { Degrees of } \\
\text { freedom }\end{array}$ & t-value & p-value \\
\hline \multirow{4}{*}{$\begin{array}{l}\text { Sponge } \\
\text { abundance }(N)\end{array}$} & Intercept & 169.282 & 17.894 & 149 & 9.460 & $<0.001$ \\
\hline & $\begin{array}{l}\text { Years from } \\
2005\end{array}$ & -4.779 & 1.040 & 149 & -4.595 & $<0.001$ \\
\hline & Site B & -63.264 & 21.526 & 12 & -2.939 & 0.012 \\
\hline & Site C & -13.688 & 22.747 & 12 & -0.602 & 0.558 \\
\hline \multirow[t]{6}{*}{ Richness $(S)$} & Intercept & 11.467 & 1.370 & 147 & 8.373 & $<0.001$ \\
\hline & $N$ & 0.029 & 0.007 & 147 & 4.005 & 0.001 \\
\hline & Site B & -1.752 & 1.931 & 12 & -0.908 & 0.382 \\
\hline & Site C & 2.029 & 2.101 & 12 & 0.966 & 0.353 \\
\hline & {$[N]:[$ Site B] } & 0.059 & 0.015 & 147 & 3.941 & 0.001 \\
\hline & {$[N]:[$ Site $\mathrm{C}]$} & 0.020 & 0.012 & 147 & 1.587 & 0.115 \\
\hline \multirow[t]{6}{*}{ Diversity $\left(H^{\prime}\right)$} & Intercept & 1.983 & 0.123 & 147 & 16.064 & $<<0.001$ \\
\hline & $N$ & -0.0004 & 0.0006 & 147 & -0.777 & 0.439 \\
\hline & Site B & 0.150 & 0.171 & 12 & 0.876 & 0.398 \\
\hline & Site C & 0.291 & 0.171 & 12 & 1.701 & 0.115 \\
\hline & {$[N]:[$ Site B] } & 0.003 & 0.001 & 147 & 2.484 & 0.014 \\
\hline & {$[N]:[$ Site C $]$} & 0.001 & 0.001 & 147 & 1.099 & 0.274 \\
\hline \multirow[t]{2}{*}{$\begin{array}{l}\text { Evenness } \\
\left(E_{1 / D}\right)\end{array}$} & Intercept & 0.429 & 0.038 & 147 & 11.155 & $<0.001$ \\
\hline & $N$ & -0.001 & 0.0002 & 147 & -4.580 & $<0.001$ \\
\hline
\end{tabular}




\begin{tabular}{|l|l|c|c|c|c|c|}
\hline $\begin{array}{l}\text { Dependent } \\
\text { variable }\end{array}$ & Coefficient & Estimate & Standard error & $\begin{array}{c}\text { Degrees of } \\
\text { freedom }\end{array}$ & t-value & p-value \\
\hline & Site B & 0.304 & 0.056 & 12 & 5.417 & $\mathbf{0 . 0 0 2}$ \\
\hline & Site C & 0.114 & 0.060 & 12 & 1.910 & 0.080 \\
\hline & {$[N]:[$ Site B] } & -0.002 & 0.0004 & 147 & -5.062 & $<\mathbf{0 . 0 0 1}$ \\
\hline & {$[N]:[$ Site C $]$} & -0.0004 & 0.0003 & 147 & -1.382 & 0.169 \\
\hline
\end{tabular}

\subsubsection{Effects of water temperature on sponge abundance and growth}

I found no significant effect of SST or SSTANOM on $N$ or $g$ (results not shown). SST anomalies were always positive in 2016, indicating a particularly warm year (Fig. A2.4). However, decomposition of the time series with moving averages of both SST and SSTANOM showed no clear temporal trend (Fig. 3.3-3.4), indicating that SST and SSTANOM have not increased at this site over the study period. Due to lack of evidence for effects of temperature on temporal changes of this sponge assemblage, and lack of clear temporal trends in sea water temperature at this station over the study period, I excluded SST and SSTANOM from further steps in this investigation.

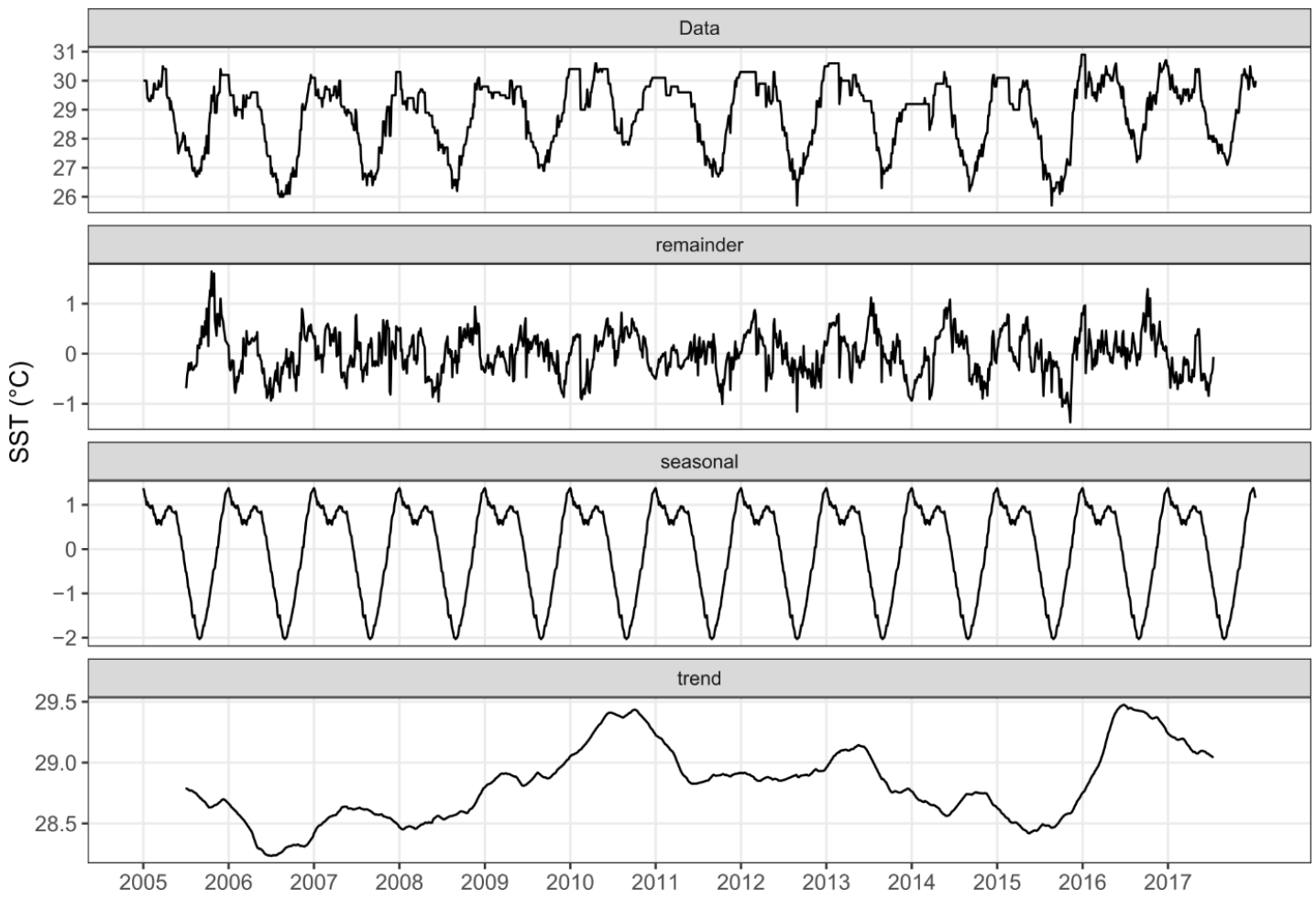

Figure 3.3. Decomposed sea surface temperature (SST) time series data. Random, seasonal, and trend component extracted with moving average decomposition. 


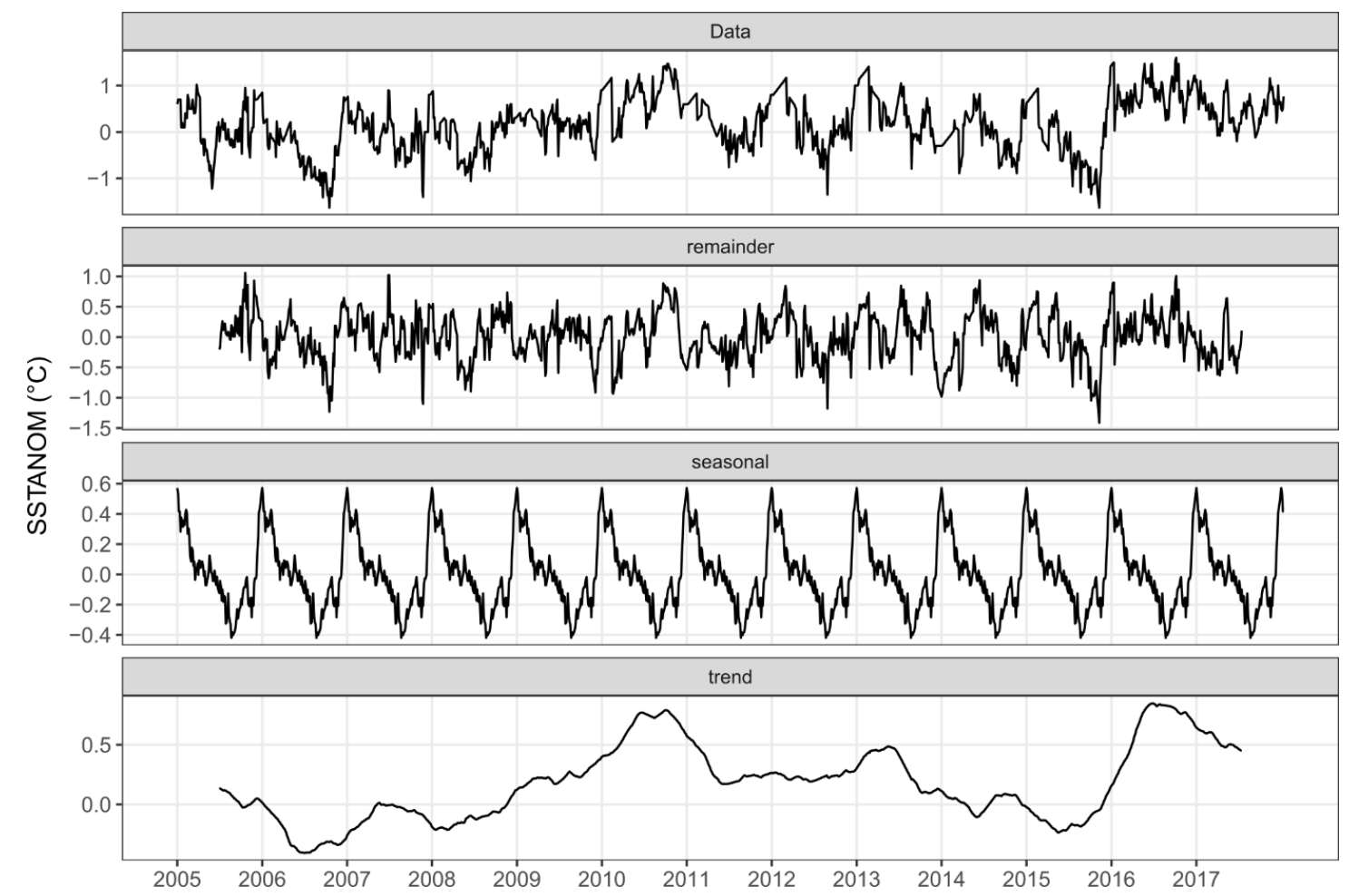

Figure 3.4. Decomposed sea surface temperature anomalies (SSTANOM) time series data. Random, seasonal, and trend component extracted with moving average decomposition.

\subsubsection{Species richness, diversity and evenness}

Fluctuations in species richness followed similar patterns to sponge abundance. Maximum mean species richness at this reef (species per quadrat \pm SE) was recorded in 2007 at the time of highest sponge abundance (22.60 \pm 0.88$)$, whereas the minimum was recorded in 2013 (12.8 $\pm 0.89)$. Assemblage evenness followed the opposite pattern, being lowest in $2007(0.29 \pm 0.02)$ and highest in $2013(0.49 \pm 0.05)$. The overall minimum number of species per quadrat recorded was 6 in 2013 (site A, quadrat 4), whereas the highest was 34 in 2009 (site C, quadrat 1). On average, species richness was highest at site $C$ in 2007 and 2009 (mean \pm SE $25.8 \pm 0.97$ species per quadrat; $25.8 \pm 2.35$ species per quadrat respectively), and lowest at site B in 2013 (10.8 \pm 0.73 species per quadrat). Mean $H^{\prime}$ was highest at site B in 2009 (2.73 \pm 0.32$)$, and lowest at site $\mathrm{A}$ in 2015 (1.66 \pm 0.18$)$. However, $H^{\prime}$ did not show temporal patterns consistent across quadrats and sites as found for $N$ and $S$.

$N$ had a site-specific positive effect on species richness $S(P<0.001)$ and a site-specific negative effect on species evenness $E_{1 / D}(P=0.002) . N$ and site had an interactive effect on species diversity $H^{\prime}(P=0.05)$, but this effect was generally weak and was statistically different from 0 only at site B. Best LMM fits are shown in Figure 3.5. Full LMM results and coefficients are reported in Table 3.1. 

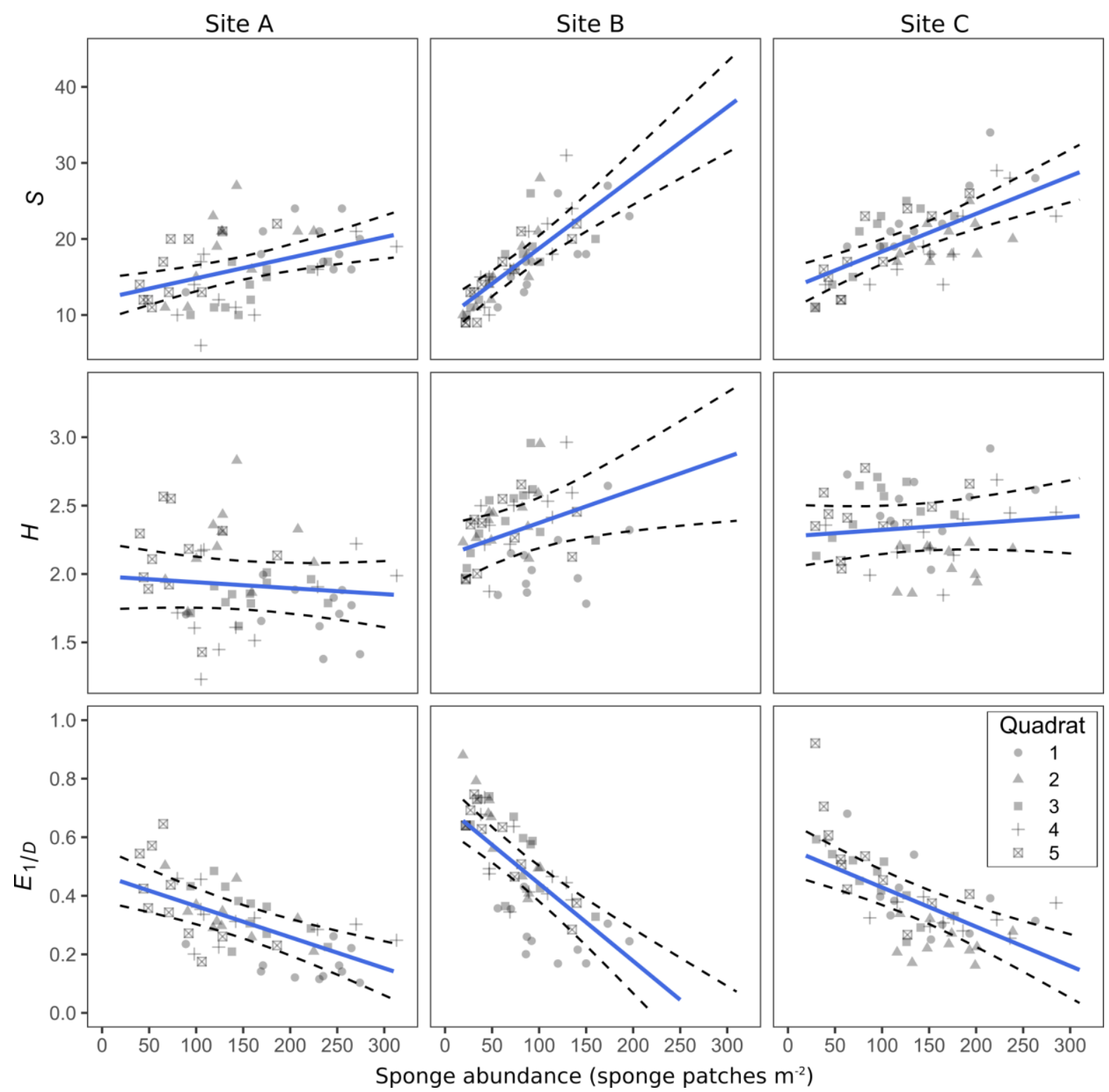

Figure 3.5. Effect plots showing the best linear mixed models (LMMs) fits for the biodiversity metrics (species richness $S$, Shannon's diversity $H^{\prime}$, and Simpson's evenness $E_{1 / D}$ ) modelled as function of sponge counts and site. Blue lines: best LMM fit; black dashed lines: 95\% confidence intervals of the model parameters; grey dots: observed data. Different shapes of the dots represent different quadrats within each site.

\subsubsection{Spatio-temporal changes in species composition}

nMDS showed that the sponge assemblage composition varied in both space and time (stress $=0.16$, Fig. 3.6-A). Quadrats from site B were partially separated from quadrats at sites A and C, which were more similar to each other. Sycon sp., Pseudoceratina sp. 1, and Protosuberites 
sp., were dominant at sites $\mathrm{A}$ and $\mathrm{C}$ and were less strongly associated with site $\mathrm{B}$, whereas some less abundant taxa (e.g. Cliona sp. and Clathrina sp. 2) were mostly found at site B (Fig. 3.6-B and Fig. A2.5; also see Fig. A2.6 for year-by-year MDS plots). Samples from the same year tended to cluster together both within and across sites, and samples from adjacent years were also relatively close to each other. Permutational MANOVA showed that time (years) explained $21 \%$ of the variation $(F=4.83, P<0.001)$, site explained $12 \%(F=13.38, P<$ $0.001)$, and the interaction between these two factors explained a further $10 \%(F=1.17, P=$ 0.031). Finally, the CAP plot highlighted a difference in the composition of the assemblage between years of high and low sponge abundance (Fig. 3.6-B). Specifically, years with high sponge abundance were characterised by high numbers of Protosuberites sp., Sycon sp. and Pericharax sp. All three are heterotrophic sponges but are taxonomically distant (Protosuberites sp. is a demosponge, while Pericharax sp. and Sycon sp. are calcareous sponges). Proportions of these taxa in the assemblage varied between sites and over time, but on average they accounted for $\sim 25 \%, 20 \%$ and $5 \%$ of total sponge abundance, respectively. Population dynamics of the most abundant sponge taxa (Tab. 3.2) during the study period can be found in the Appendix 2 (Fig. A2.5).

Table 3.2. List of most common taxonomically identified sponge taxa from Hoga Island, Wakatobi, Indonesia. Of the sponge taxa that, summed, comprised $>90 \%$ of the total sponge abundance in each photoquadrat ( 30 of the total 141 taxa), the following 25 could be identified to genus level or lower. Abbreviations used in the Canonical Analysis of Principal coordinates (CAP) plot (Fig. 3.6-B) are reported in the taxon column. Mean abundances for this reef during the study period are reported.

\begin{tabular}{|l|l|c|c|}
\hline $\begin{array}{l}\text { Taxon (CAP } \\
\text { abbreviation) }\end{array}$ & Family & Class & $\begin{array}{c}\text { Mean } \pm \text { SE sponge } \\
\text { abundance (sponge } \\
\text { patches } \mathbf{~ m}^{-2} \text { ) }\end{array}$ \\
\hline Aaptos sp. & Suberitidae & Demospongiae & $1.02 \pm 0.10$ \\
\hline Axinyssa sp. & Halichondriidae & Demospongiae & $0.27 \pm 0.06$ \\
\hline Chalinula milnei & Chalinidae & Demospongiae & $1.81 \pm 0.24$ \\
\hline Chalinula $\mathrm{sp}$. & Chalinidae & Demospongiae & $0.47 \pm 0.13$ \\
\hline
\end{tabular}




\begin{tabular}{|c|c|c|c|}
\hline $\begin{array}{l}\text { Taxon (CAP } \\
\text { abbreviation) }\end{array}$ & Family & Class & $\begin{array}{c}\text { Mean } \pm \text { SE sponge } \\
\text { abundance (sponge } \\
\text { patches } \mathbf{m}^{-2} \text { ) }\end{array}$ \\
\hline $\begin{array}{l}\text { Chelonaplysilla } \mathrm{sp} .1 \\
\text { (Che) }\end{array}$ & Darwinellidae & Demospongiae & $3.18 \pm 0.22$ \\
\hline Cinachyrella sp. 2 (Ci2) & Tetillidae & Demospongiae & $0.33 \pm 0.05$ \\
\hline Cinachyrella sp. 3 & Tetillidae & Demospongiae & $0.32 \pm 0.05$ \\
\hline $\begin{array}{l}\text { Clathria (Microciona) } \\
\text { mima }\end{array}$ & Microcionidae & Demospongiae & $0.35 \pm 0.08$ \\
\hline Clathrina sp. 1 & Clathrinidae & Calcarea & $0.17 \pm 0.08$ \\
\hline Clathrina sp. $2(\mathrm{Cl} 2)$ & Clathrinidae & Calcarea & $2.22 \pm 0.41$ \\
\hline Clathrina sp. 4 (Cl4) & Clathrinidae & Calcarea & $0.44 \pm 0.08$ \\
\hline Clathrina sp. 5 & Clathrinidae & Calcarea & $0.43 \pm 0.07$ \\
\hline Clathrina sp. 6 & Clathrinidae & Calcarea & $0.09 \pm 0.03$ \\
\hline Cliona sp. (Cli) & Clionaidae & Demospongiae & $0.15 \pm 0.08$ \\
\hline Dendrilla sp. (Den) & Darwinellidae & Demospongiae & $1.45 \pm 0.19$ \\
\hline Haliclona sp. (Hal) & Chalinidae & Demospongiae & $4.55 \pm 0.42$ \\
\hline $\begin{array}{l}\text { Lamellodysidea herbacea } \\
\text { (Ldy) }\end{array}$ & Dysideidae & Demospongiae & $3.89 \pm 0.44$ \\
\hline Niphates sp. 1 & Niphatidae & Demospongiae & $0.21 \pm 0.05$ \\
\hline Niphates sp. 2 (Ni2) & Niphatidae & Demospongiae & $0.88 \pm 0.1$ \\
\hline Niphates sp. 3 & Niphatidae & Demospongiae & $0.05 \pm 0.04$ \\
\hline Pericharax sp. (Per) & Leucettidae & Calcarea & $7.33 \pm 0.60$ \\
\hline $\begin{array}{l}\text { Placospongia } \\
\text { melobesioides }\end{array}$ & Placospongiidae & Demospongiae & $0.74 \pm 0.11$ \\
\hline
\end{tabular}




\begin{tabular}{|l|c|c|c|}
\hline $\begin{array}{l}\text { Taxon (CAP } \\
\text { abbreviation) }\end{array}$ & Family & Class & $\begin{array}{c}\text { Mean } \pm \text { SE sponge } \\
\text { abundance (sponge } \\
\text { patches } \mathbf{~ m}^{-2} \text { ) }\end{array}$ \\
\hline Protosuberites sp. (Pro) & Suberitidae & Demospongiae & $32.27 \pm 2.73$ \\
\hline $\begin{array}{l}\text { Pseudoceratina } \text { sp. } 1 \\
\text { (Pse) }\end{array}$ & Pseudoceratinidae & Demospongiae & $5.50 \pm 0.58$ \\
\hline \begin{tabular}{l} 
Sycon sp. (Syc) \\
\hline
\end{tabular} & Sycettidae & Calcarea & $20.30 \pm 1.79$ \\
\hline
\end{tabular}
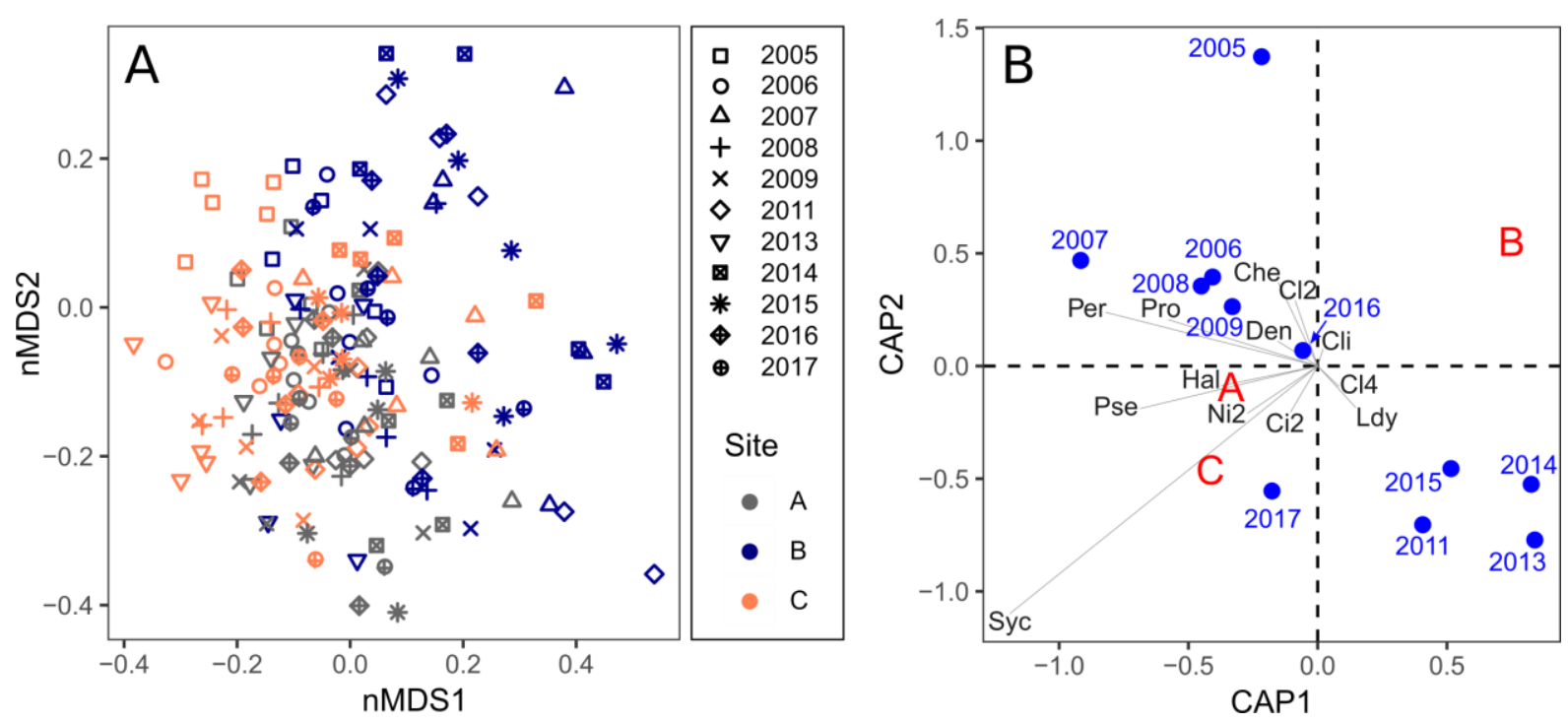

Figure 3.6. Spatial and temporal variation of sponge assemblage composition. A: Non-metric Multi-dimensional Scaling (nMDS) plot for the sponge assemblages at the three sites between 2005 and 2017. Each sample corresponds to a quadrat. Symbols indicate the year, colours the site. B: Canonical Analysis of Principal coordinates (CAP) plot. Years and sites represent the centroid for each set of samples. For visualisation purposes, only the taxonomically identified taxa that varied the most between sites and years are plotted (but all taxa were included in the analysis). See Table 3.2 for species abbreviations. 


\subsection{Discussion}

\subsubsection{Temporal changes in sponge abundance}

Considerable temporal variation was evident in the abundance and species composition of the sponge assemblage on this Indonesian coral reef over a 13-year period. I found that a subset of species had large population variability and determined the magnitude of the fluctuations in total sponge abundance. No effect of sea surface temperature (SST) and SST anomalies was detected on sponge abundance and interannual sponge growth at this location. Biodiversity also changed considerably over the studied period, with species richness generally increasing with sponge abundance. However, due to a few dominant species, assemblage evenness was lowest when total sponge abundance was highest.

In the Caribbean, rapid declines in sponge assemblage abundance due to widespread mortality events have been reported and these may or may not be followed by recovery. For example, Butler et al. (1995) found that $80 \%$ of sponges were decimated in Florida Bay after being exposed to a cyanobacterial bloom in 1991, and the remaining sponges died in a second bloom in 1992. Subsequently, Stevely et al. (2016) showed that the same sponge assemblages displayed signs of overall long-term recovery, while Wulff (2006b) reported a $42.6 \%$ decline in sponge volume on a Panamanian reef over 14 years, but here the decline was not followed by recovery. In the present study, the Indo-Pacific sponge assemblage showed a continual decline over about 7 years from 2008, in contrast to the abrupt drops caused by acute disturbance observed in some earlier studies (e.g. Stevely et al. 2011). This suggests that the changes in sponge abundance reported in the present study were unlikely to be driven by an acute disturbance, like the sudden drop reported by Stevely et al. (2011). However, total sponge abundance followed the same pattern of fluctuation both within and across the three sites, suggesting that independent stochastic variation at a scale of $100 \mathrm{~s}$ of meters was also unlikely to have driven the observed demographic trends.

A direct and important consequence of the observed variability is that shorter-term sampling within the studied period could have yielded different information on sponge abundance on this coral reef. For example, if sampling had occurred solely in 2007 (year of maximum mean sponge abundance), it would have shown almost 3 times as many sponge patches as if it had occurred only in 2014 (year of minimum mean sponge abundance). The rarity of long-term studies of sponge dynamics and ecology highlights the need to complement studies considering 
spatial variability across environmental gradients at single time-points, with the continuous and regular monitoring of assemblages.

\subsubsection{Temporal changes in taxonomic composition and biodiversity}

The sponge assemblage from the Wakatobi was highly diverse, with a total of 141 sponge taxa recorded over 13 years in an area of only $15 \mathrm{~m}^{2}$ (see Bell 2007, Bell and Smith 2004 for a list of sponge families at Hoga Island, and Table A2.1 for the complete list of taxonomically identified taxa). Such sponge diversity is comparable to previous reports from other parts of Indonesia, or higher when considering that it was recorded at one single reef. Cleary and de Voogd (2007) reported 151 taxa from 9 reefs in the Spermonde Archipelago; de Voogd and Cleary (2008) counted 118 sponge species at 30 reefs in Thousand Islands; de Voogd et al. (2009) reported 168 sponge taxa from 18 sites in NE Kalimantan; and Calcinai et al. (2017) reported 94 sponge species from 26 sites in North Sulawesi. However, most of these previous studies did not survey small encrusting sponges. When sponges were most abundant, a few taxa were dominant (e.g. Protosuberites sp., Sycon sp., and Pericharax sp.). The decline of these taxa determined the observed lower overall sponge abundance, and their population variability caused the composition of the overall assemblage to change over time.

Studies that describe and account for temporal variation of assemblage structure are rare, although tropical sponge assemblages have been reported to undergo rapid fluctuations and species-specific dynamics. Wulff (2006b) documented a $51 \%$ decline in sponge species richness at a reef in Panama over a 14-year period, with massive growth forms suffering disproportionate losses. Similarly, Wulff (2013) showed that sponges in an assemblage on the Belize Barrier Reef that had been monitored from 2006 to 2012 had species-specific responses and recovery patterns to cyanobacteria-induced mortality. These studies show examples of taxa-specific response to the same disturbance. However, different sponge species have also been found to have similar temporal dynamics in response to a perturbation. For example, in a long-term transplantation experiment, Wulff (2017) observed that all 12 ecologically and phylogenetically different sponge species responded by growing faster under higher picoplankton concentrations. Similarly, taxonomically unrelated species of the Indonesian sponge assemblage described in the present study showed similar temporal dynamics, although the magnitude of the fluctuations was different for different species. Similar temporal dynamics of different taxa suggest that the observed dynamics are not stochastic fluctuations and may be caused by a common driver (see discussion below). 
Because ecosystem that can support abundant populations often can, according to general ecological theory, support a higher number of species (Wright 1983), species richness often increases with abundance (Bock et al. 2007). However, richness and evenness of biodiverse communities can be negatively correlated when those few species that benefit the most from the environmental conditions become dominant, and in doing so reduce the evenness of the community (Stirling and Wilsey 2001, Zhang et al. 2012). In this assemblage, the negative linear relationship between evenness and abundance was due to the dominance of some abundant taxa (e.g. Protosuberites sp., Sycon sp., Pericharax sp.). Whilst their abundance fluctuated, these dominant taxa were present in the community even at times of low sponge abundance. This means that, although these species had the highest absolute population variability, other less common sponge taxa appeared in years of high sponge abundance, thus contributing to species richness. In the context of potential increases in sponge abundance on future coral reefs (see Chapter 2, Bell et al. 2018a), it will be important to monitor the species richness and evenness of sponge assemblages. Regime shifts towards sponge reefs may be the result of the disproportionate growth of the populations of few dominant taxa, which would manifest as abundant but uneven sponge assemblages.

Spatially, the assemblage also showed differences in abundance, composition, and biodiversity metrics between sites. Site B generally had lower N than Sites A and C and also had a different assemblage composition. This difference is likely attributed to different localscale environmental conditions at the reef wall at Site B, compared to Sites A and C. The importance of local-scale factors in affecting sponge abundance and zonation patterns has been documented previously. For example, different exposure to light, reef aspect, and sedimentation can have an important impact on sponges and other benthic organisms (Cheshire and Wilkinson 1991, Bell and Barnes 2000a, Carballo 2006, Bell et al. 2015b, McGrath et al. 2017).

\subsubsection{Potential causes of temporal variability}

Temporal dynamics of sponge assemblages can be controlled by large-scale climatic factors and by local environmental drivers (Carballo et al. 2008). Although the consistency of sponge population dynamics within and across all sites suggested that stochastic fluctuations were not the only driver of this sponge assemblage, I could not detect any effect of seawater temperature on sponge abundance and population growth. There is almost no information on how IndoPacific sponge assemblages respond to changes in water temperature. Powell et al. (2014) reported temperature accounted for only $4 \%$ variation of sponge assemblage composition 
across sites. A temperature-induced bleaching event was documented for the bioeroding sponge Cliona aff. viridis on Hoga Island, following a period of above-average water temperature in May 2016 (Marlow et al. 2018b). However, I suggest that if water temperature had an effect on sponge demographics at this site during the study period, the effect was only weak and could not be detected due to interannual population variability. McCain et al. (2016) show that resurvey and time-series studies struggle to detect chronic effects of climate change stressors on natural population dynamics, due to stochastic population variability, unless the population is highly stable, or the stressor has a very strong impact. Whilst these authors simulated the effects of climate change on natural populations on a longer time scale than the present study (3-5 decades), McCain et al. (2016) also showed that survey length is less relevant than interannual population variability in the accurate detection of climate-driven changes on population abundance. Moreover, investigating the relationships between larger-scale environmental data (e.g. daily satellite measurements of sea-surface temperature) and local biological data is particularly challenging. Averaging the fine-scale variable (e.g. SST) to match the resolution of the less frequently sampled parameter (e.g. sponge abundance) causes a loss of information on its temporal variability (Ferguson et al. 2017). That is, the mismatch in the resolution of two time-series data can hinder the ability to detect relationships between them. If changes in sponge abundance of this assemblage were caused by short-term thermal anomalies like the one reported by Marlow et al. (2018b), averaging temperature data may have made such anomalies undetectable by my analysis.

I did not investigate the role of local environmental covariates or biotic interactions in regulating sponge abundance and biodiversity in this study, because such covariates have not been regularly sampled at this site. Previous research in the Wakatobi showed that sedimentation and light availability are the main environmental factors influencing spatial patterns of sponge biodiversity (Bell and Smith 2004) and functional diversity (Bell 2007). Sedimentation in the Wakatobi was found to reduce sponge diversity (Powell et al. 2014, Biggerstaff et al. 2017b), to limit bioerosion rates of boring sponges (Marlow et al. 2018c), and to cause an energetic cost associated with mucus production (McGrath et al. 2017). Settled sediment data was available for Buoy 3 for years 2014 and 2015 (from Marlow et al. 2018c), but sedimentation data from adjacent years would be unlikely to show changes in sedimentation rates that are strong enough to drive changes in sponge demographics, mostly due to the relatively low levels of settled sediments on this vertical reef compared to more degraded sites (Marlow et al. 2018c). 
The effect of spongivory on sponge assemblages has been primarily studied in the Caribbean (reviewed in Pawlik et al. 2018). There is debate over whether bottom-up food limitation (Lesser and Slattery 2013, Slattery and Lesser 2015) or top-down biotic interactions are the most important drivers for sponges (Wulff 2006a, Loh and Pawlik 2012, Pawlik et al. 2013, 2015, Wulff 2017). While recent evidence points towards a synergistic action of top-down and bottom-up controls (Wulff 2017), such interactions may be restricted to lagoonal habitats, with sponge assemblages on fore-reefs likely to be structured by predation (Pawlik et al. 2018). The role of top-down control on sponge assemblages in the Indo-Pacific remains largely unexplored. Previous research from the Wakatobi reports a weak effect of spongivorous fish abundance on the composition of sponge assemblages (6\% of variation, Powell et al. 2014). Powell et al. (2015) found that nudibranchs and at least 16 fish species engage in trophic interactions with sponges in the Wakatobi region, however excluding predators from sponges with cages showed no effects on the abundance of non-cryptic sponges. Further research is needed to elucidate the role of spongivory in this region, but from these studies it seems unlikely that predation alone could drive the broad temporal changes in sponge abundance of this assemblage.

\subsubsection{Implications of assemblage variability for coral reef monitoring}

Temporal fluctuations in the abundance and diversity of sponges can have major implications for the ecological functioning of coral reefs. Functions carried out by sponges, such as water filtering (de Goeij et al. 2008) and interactions with other benthic organisms and predators (Wulff 2006a) will change with variation in sponge abundance. The coupling between sponge abundance and sponge ecological roles has important implications for resource management and conservation, especially if sponges become more abundant or dominant on future coral reefs (Bell et al. 2013). Sponges are abundant at this reef, with sponge percentage cover estimated at $29.5 \pm 7 \%$ shortly before the start of the study period (Bell and Smith, 2004). A sponge reef that fluctuates between states of high and low sponge abundance is also likely to be characterised by a different associated food web, with potential implications for fisheries and other ecosystem services. Although trophic interactions of sponges in the Indo-Pacific remain largely unknown, evidence of spongivory from several fish species and from nudibranchs (Powell et al. 2015) suggests that strong temporal changes in abundance and taxonomic composition of sponge assemblages may result in a modified diet for opportunistic feeders (Wulff 2006a), and as a consequence potential alteration of food web dynamics (Bell et al. 2013). Sponges in the Indo-Pacific have also been found to overgrow live corals (de 
Voogd et al. 2004), occupy dead coral substrate (Rossi et al. 2015), and bioerode the reef framework (Marlow et al. 2018a). Therefore, an increase in the abundance of some species may have negative impacts on live reef-building corals, on the availability of free space, and on reef structure. Fluctuations in sponge abundance may also have effects on benthic composition due to symbiotic relationships between some sponge species and other sessile reef organisms in the Indo-Pacific, like coralline algae (Calcinai et al. 2006) and soft corals (Calcinai et al. 2013).

Understanding the causes of temporal variability in sponge assemblages will play an important role in projecting how they may respond to environmental change. The high temporal variability and species-specific dynamics of this sponge assemblage could hamper the detection of environmental impacts on sponges as a single group over sub-decadal scales. However, disentangling the relative contributions of single species to changes in abundance and taxonomic composition of the assemblage over time allowed for an estimation of speciesspecific population variability. Features like long survey time, consistent sampling protocol and characterization of specific population variability are needed to clarify our understanding of the effects of perturbations on natural populations (McCain et al. 2016).

The annual sampling design of the present study did not allow us to capture intra-annual variations in sponge abundance or assemblage composition. Although there is limited information on the life history of sponge species from this region, calcareous sponges are known to have short life spans (weeks to months, Padua et al. 2016) and seasonal changes in metabolic rates (Burlando et al. 1992). Since the calcareous sponges Sycon sp. and Pericharax sp. were abundant at this reef, it is possible that sponge abundance and diversity of this assemblage experienced seasonal changes that the sampling design could not detect.

Monitoring coral reefs in remote locations is often expensive and logistically challenging, and the optimal duration and design of a sponge monitoring program for a sponge assemblage depends on the questions and resources at hand (Bell et al. 2017). I show that a monitoring protocol that: 1) uses permanent monitoring units; 2) is repeated over multiple years, ideally 5 10; and 3) is carried out with consistent techniques, allowed me to study temporal changes in abundance and biodiversity of this Indo-Pacific sponge assemblage. To avoid mismatch in the spatial or temporal scale of environmental and biological data, I suggest that environmental covariates (like water temperature, sedimentation, and other drivers that may impact the studied sponge fauna) be measured in situ and accompany sponge surveys, where possible. Limitations of using sponge abundance as a metric for sponge monitoring have been discussed in Bell et 
al. (2017), and include the possible confusion between fragmentation of a sponge patch and recruitment, and between fusion of sponge patches and mortality. Moreover, temporal dynamics of sponge abundance and biomass or percent cover may be different in some cases. For example, if one larger sponge patch grows in size while no increase in total sponge abundance occurs, sponge percent cover in the quadrat may increase while total sponge abundance stays the same. While repeated counts of sponge patches are a time-efficient, noninvasive method of quantifying temporal variation of a sponge assemblage, other measures of sponge abundance to complement sponge counts are desirable, as long as they can be obtained with non-destructive sampling (e.g. cover or volume estimates) and are logistically practical.

This Chapter highlights the value of long-term monitoring of sponge assemblages and natural systems in general. The importance of accounting for short-term seasonal variation in the monitoring of sponge assemblages has been highlighted by Berman and Bell (2016). Here I show that longer-term dynamics (encompassed by annual sampling) can also be highly variable. Mean total sponge abundance on this reef went through phases of increase as well as decline, decreasing by as much as $63 \%$ over a 7 -year period, with the species composition of the assemblage also varying substantially. Thus, when drawing conclusions from short-term ecological studies, it is imperative to put results into a temporal context, because data from one single time point can lead to misleading assessments of assemblage abundance and biodiversity.

The knowledge gained from this detailed analysis of small-scale spatial patterns and temporal dynamics of tropical sponge assemblages guided the conceptualisation and qualitative validation of the Atlantis model developed as part of this thesis (Chapter 6). Atlantis is a complex ecosystem model where organisms are often aggregated into more or less taxonomically resolved groups (Audzijonyte et al. 2019). Moreover, Atlantis is often applied to large geographic regions (examples include the Gulf of Mexico, Ainsworth et al. 2015, and the West Coast of the United States, Marshall et al. 2017). The sheer spatial scale and ecological complexity of most Atlantis models inevitably mask granular patterns, like the local fluctuations of complex sponge assemblages reported in this chapter. However, sponges are of central interest in the Atlantis application presented in this thesis. Ecosystem models are bound to sacrifice the realism of fine-scale dynamics of the modelled species (Collie et al. 2014). Therefore, detailed studies of the organisms of central interest in the model are desirable to supplement the large-scale, taxonomically coarse in-model representation of such organisms. 
Chapter 4. Decadal stability and interannual variability of benthic organisms on an Indo-Pacific coral reef 


\begin{abstract}
The availability of free space is an important driver of the temporal dynamics of sessile invertebrates on coral reefs. The ability of reef organisms to occupy space that becomes available, for example, after a mortality event, influences the successional patterns of benthic fauna on reefs, and can result in regime shifts to alternative states. Increased dominance of algae and, in some cases, sponges has been documented on many reefs across the world, but how these groups benefit from non-colonised substrate on the reef is unclear. In this Chapter, I described the temporal dynamics of benthic organisms on an Indonesian coral reef between 2006 and 2017, and investigated the effects of free space on benthic cover at subsequent sampling events. In contrast with other reefs in the Indo-Pacific where corals have been declining, corals were dominant and stable over time at this site (mean \pm SE percentage cover $42.69 \pm 1.86 \%$ ), and no other benthic group became more abundant than corals over the study period. Percentage cover of turf algae and sponges showed larger interannual variability than corals and crustose coralline algae $(\mathrm{CCA})(P<0.001)$, indicating that these groups are more dynamic over short temporal scales. Bare substrate was a good predictor of turf cover in the following year (mean effect $0.199,95 \%$ CIs [0.036, 0.362]). Cover of algal groups combined with bare space was a good predictor of CCA cover the following year, and of sponge cover the following year at one of the sites. These results suggest that CCA may overgrow existing patches of algal turf, although my data did not capture the mechanisms by which succession from turf to CCA occurred. The results of this chapter indicate that turf algae in the Indo-Pacific rapidly occupy free space when this becomes available, and that other benthic groups are likely not limited by the availability of bare substrate, but may overgrow already fouled substrates.
\end{abstract}

\title{
4.1. Introduction
}

The abundance of coral reef organisms shows a wide range of temporal patterns, including long-term trends of change and short term (interannual or seasonal) fluctuations (Adjeroud et al. 2002, Shenkar et al. 2008, Lamy et al. 2016, Chapter 3). Over the last few decades, coral reefs have been impacted by many local and global stressors, which have often caused loss of coral cover and changes in coral reef benthic communities (e.g. Hoegh-Guldberg et al. 2007, Hughes et al. 2017a, Cheal et al. 2017, Brown and Hamilton 2018). Indo-Pacific coral reefs are a global hotspot of marine biodiversity (Roberts et al. 2002). However, reefs in this region have also suffered a decline in coral cover, due to pollution (Edinger et al. 1998), increased water 
temperature (Graham et al. 2015, Heron et al. 2016), cyclones (Wantiez et al. 2006), extreme thermal events (Adjeroud et al. 2002), and outbreaks of the corallivorous crown-of-thorns starfish Acanthaster planci (Kayal et al. 2012, Baird et al. 2013). Loss of coral cover in the Indo-Pacific has been estimated at $1 \%$ per year between the early 1980s and 2003, and closer to $2 \%$ in the early 2000s (Bruno and Selig 2007). Indo-Pacific reefs are also projected to experience severe bleaching at increasing frequency in the future (Meissner et al. 2012, Hughes et al. 2017b).

When corals die, barren substrate becomes available for larvae of benthic organisms to settle, or for neighbouring individuals or colonies to expand into. After coral mortality and the early establishment of microbial films, macroalgae or filamentous turf algae can settle on the dead coral substrate (see Hughes 1994). Colonization of free substrate from algae may be followed either by a long-term recovery of corals or by the establishment of an algal-dominated state (see Mumby et al 2007, Dudgeon et al. 2010, Jouffray et al. 2015). The establishment of feedback mechanisms that prevent the ecosystem from reverting to the original coraldominated state are responsible for the permanent shift to an alternative stable state (Suding et al. 2004). In case of shifts to algal reefs, these feedbacks include macroalgal-mediated preemption of substrate for corals to recruit on (Johns et al. 2018), and the decoupling between grazing pressure from herbivores and macroalgal abundance (Mumby et al. 2007), for example due to the establishment of unpalatable macroalgae (Scheffer et al. 2001). However, identifying changes in the abundance of benthic organisms over time is important, as transient changes in benthic dominance may be followed by permanent system hysteresis if the perturbation that caused them persists, and if ecological feedbacks are established (Mumby et al. 2007).

While shifts towards algal dominance are the most common, increased dominance of other benthic organisms has also been documented from reefs around the world (Norström et al. 2009), including soft corals (Cruz et al. 2015), ascidians (Roth et al. 2018, Tebbett et al. 2019), and sponges. Increased dominance of sponges on coral reefs has been documented for the Coral Triangle (Powell et al. 2014, Biggerstaff et al. 2017b), the Mariana Islands (Schils 2012), Palmyra Atoll (Knapp et al. 2013, Knapp et al. 2016), Brazil (Kelmo et al. 2013), and many reefs across the Caribbean (e.g. Ward-Paige et al. 2005, López-Victoria and Zea 2005, McMurray et al. 2010). Coral reef sponges are strong benthic competitors (Suchanek and Carpenter 1983, Aerts 1998, Bell and Barnes 2003, Rützler 2004), and they compete for space on the reef both through direct competition with hard corals (Hoeksema and de Voogd 2012, Rossi et al. 2015, Wang et al. 2015, Elliott et al. 2016) and by excavating dead coral skeleton 
and carbonate substrate (reviewed in Schönberg et al. 2017). In ecosystems that are subject to prolonged perturbation some sponge species may become more abundant to the expense of corals and other reef benthic organisms (for example some sponges on sedimented reefs, see Biggerstaff et al. 2015, 2017a,b), and an increased sponge abundance may be followed by a permanent shift towards sponge dominance if ecological feedbacks like the pre-emption of space for corals to recover are established (Norström et al. 2009).

While there is evidence of algae and other invertebrates becoming dominant on some coral reefs, the temporal dynamics of changes in benthic dominance are not always well described. Studies have investigated early succession of benthic colonisers on artificial substrates in the Indo-Pacific (e.g. Bailey-Brock 1987, Boschetti 2016, Smith et al. 2010, Plass-Johnson et al. 2016), but settlement and early succession studies generally cannot capture how established neighbouring organisms advance into natural bare substrate. In particular, little is known of how algae and sponge take advantage of free substrate becoming available on the reef after coral mortality occurred. Most research on horizontal advancement of sponges into dead substrate overgrown by algae has been carried out on clionaid bioeroding sponges in the Caribbean (López-Victoria and Zea 2005, López-Victoria et al. 2006), and on the Great Barrier Reef (Fang et al. 2017). Studies considering direct competition between coral reef sponges and algae have also focused on boring sponges (also mostly from the Caribbean), showing that macroalgae in general curtail sponge growth by pre-empting free space (González-Rivero et al. 2011, 2012, 2016). However, little information exists on how diverse sponge assemblages, which also include non-bioeroding sponges, and diverse algal groups, benefit from free substrate. Moreover, some sponge taxa are capable of using substrate occupied by other organisms (rather than only bare substrate) for settlement and expansion. Larval settlement of some Great Barrier Reef sponges is induced by crustose coralline algae (CCA) (Whalan et al. 2012), and some sponge taxa have been shown to form epibioses with turf algae (Ribeiro et al. 2018). However, relationships between sponges and CCA or turf algae in the Indo-Pacific have not been investigated.

The Wakatobi Marine National Park in Southeast Sulawesi (Indonesia) is a biodiversity hotspot within the Coral Triangle (Clifton et al. 2010). Similar to other Indo-Pacific locations, coral cover has declined across the Wakatobi over the last 15 year (Clifton and Unsworth 2010, Gouraguine et al. 2019). While the reported decline in coral cover did not coincide with an increase in macroalgae (Curtis-Quick 2013), a shift towards a sponge-dominated state has been reported from one site in the Wakatobi that is subjected to heavy sedimentation and local 
anthropogenic pressure (Powell et al. 2014, Biggerstaff et al. 2017b). However, there is a paucity of information on long-term dynamics and interannual variability of sponges on nondegraded reefs in the area (but see Chapter 3), and on whether an increase in other benthic groups has occurred alongside the documented decline in corals (Gouraguine et al. 2019).

When studying coral reef benthic communities, long-term studies with annual sampling are important to capture temporal variability of abundance and diversity (Chapter 3 ). In order to detect long-term changes in the coral reef benthic community composition of a coral reef in the Wakatobi, and to determine how different benthic organisms occupy free space that becomes available on the reef after coral mortality, I analysed percentage cover data from permanent reef quadrats between 2006 and 2017. The specific aims of this study were to: 1) investigate decadal changes in the abundance of sessile organisms on a reef wall; 2) investigate temporal changes in community structure of the benthos at this site; 3) quantify interannual variability in organism abundance; and 4) to identify the effects of free space availability on the reef at one census point on the percentage cover of benthic organisms at the following census.

\subsection{Materials and Methods}

\subsubsection{Study site and data acquisition}

The study location was Buoy 3, the same vertical reef wall off the South-West coast of Hoga Island presented in Chapter 3 (Wakatobi Marine National Park, South-East Sulawesi, Indonesia; Fig. 4.1). At this location, three sites were established in 2005, approximately 100 $\mathrm{m}$ from each other (see Chapter 3 for details). Due to morphology of the reef, sites A and B were established on the vertical wall $\left(90^{\circ}\right)$, whereas site $\mathrm{C}$ was slightly inclined $\left(\sim 80^{\circ}\right)$. At each site, 5 replicate $1 \mathrm{~m}^{2}$ permanent quadrats were haphazardly placed on the vertical wall, at a depth of 10-12 m and along a reef tract of approximately $10 \mathrm{~m}$. Permanent quadrats were chosen in order to monitor the same plots of reef over time. In years from 2006 to 2008, and from 2014 to 2017, underwater photographs of the quadrats were taken by the same observer between June and July each year. Data from the 2009-2013 period could not be collected due to logistical reason or failure of the photographic equipment. Pictures of $25 \times 25 \mathrm{~cm}$ subquadrats were taken to provide a higher resolution of the reef organisms (16 images per quadrat). Because the monitoring effort spanned across more than a decade, with new and better photographic equipment becoming available over time, photographs were taken with 
four different camera setups. However, because of the relatively coarse taxonomic detail of the benthic categories considered in the present study (see below), differences in camera resolution had negligible effects in the consistency of the identification of the substrate from the pictures. The four camera setups were: 1) Sony Cyber-Shot with strobe light; 2) Nikon D70, $60 \mathrm{~mm}$ macro lens, Ikelite DS50 strobe light; 3) Nikon D200, 60 mm macro lens, Ikelite DS50 strobe light; 4) Nikon D800, 60 mm macro lens, Ikelite DS50 strobe light. All pictures were taken with the camera lens at $30-40 \mathrm{~cm}$ from the substrate.

The diversity of sessile invertebrates at this site is high, with 141 reported sponge taxa (Chapter 3), and many species of (mostly encrusting) scleractinian corals, crustose coralline algae (CCA), turfing filamentous algae, macroalgae, ascidians, and octocorals. A total of 8 benthic categories were used to describe the substrate in the photographs. Benthic organisms were grouped into 5 taxonomic groups: hard corals, sponges, algae (macroalgae and turf), CCA, other benthic invertebrates (mostly octocorals, ascidians, bryozoans and hydrozoans). Free space suitable for colonisation (defined as bare rock or coral rubble not occupied by any visible organism) was also identified in the photographs. Importantly, due to the vertical aspect of the reef wall and to the prevalence of encrusting coral growth forms at this location, coral rubble was not an abundant component of the bottom. Abiotic substrate not suitable for growth (sand and silt) was also identified. The category 'Undetermined' was used to identify substrate that was visible in the photographs, but that was impossible to identify as any of the 5 live groups or 2 abiotic categories. In addition to the 8 benthic categories, a ninth category named "Quadrat / shade" was attributed to areas of the photograph that included the quadrat frame, or shaded areas where it was not possible to see the substrate. This category was not used in the percentage cover calculations. 


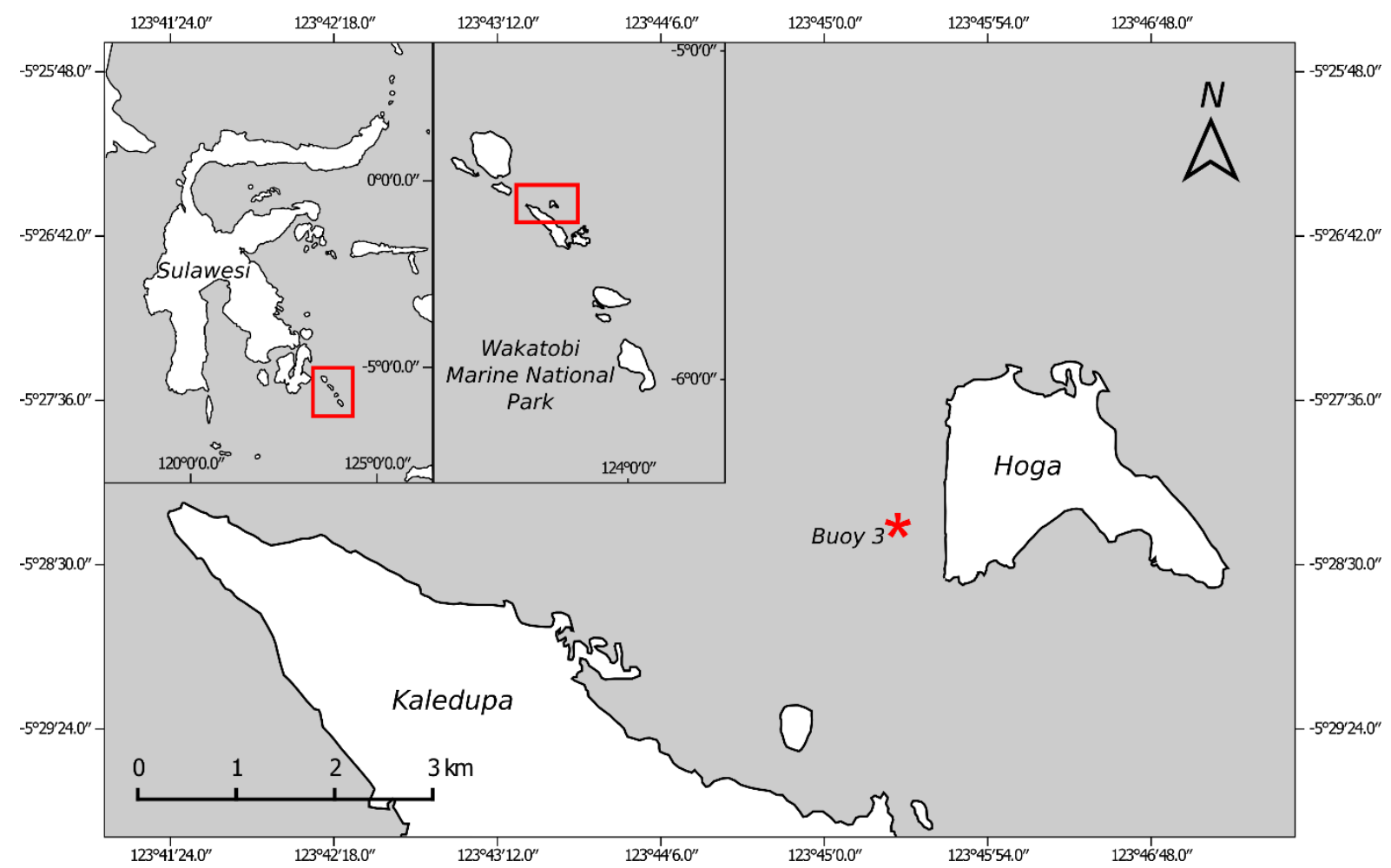

Figure 4.1. Map of Hoga Island, with insets showing the location of the Wakatobi National Marine Park and Sulawesi. The star indicates the location of the study site off Hoga Island.

Although different taxa within the same benthic group can show very different population dynamics (Chapter 3, Rovellini et al. 2019), the focus of this study was on the temporal dynamics of the main aggregated benthic groups. Furthermore, because our focus was on the interactions between hard corals, sponges and algae, other benthic invertebrates that were not as common as the three main benthic groups like octocorals, ascidians, bryozoans and hydrozoans were aggregated into one group. Turf algae and macroalgae were also aggregated into one algal group, due to the extreme rarity of macroalgae at this site.

Percentage cover was chosen as a measure of abundance for the benthic groups. While percentage cover fails to capture three-dimensional structure (i.e. it omits the vertical dimension of benthic organisms), the majority of benthic taxa at this reef was encrusting, with branching corals and complex sponge growth forms being rare. Percentage cover was thus considered an appropriate measure of abundance for the purpose of this study.

Cover of the benthic category in the permanent plots was measured with random point count methodology, a technique used to estimate population statistics of marine benthos (e.g. Carleton and Done 1995, Murdoch and Aronson 1999). In this methodology, random points 
are overlaid to the photograph of the benthos, and each point is assigned to the benthic category that occupies the underlying substrate. Percentage cover of each benthic category is then estimated as the proportion of the number of points that are assigned to that category in relation to the total number of points that are overlaid to the picture. The process was carried out with the Coral Point Count with Excel extension software (CPCe, Kohler and Gill 2006). Trials were carried out to determine the minimum number of points per picture that were necessary to accurately measure the percentage cover of the 8 benthic categories. Three randomly selected $25 \times 25 \mathrm{~cm}$ images were analysed with 20,40, 60, 80, 100, and 120 randomly assigned points per image. Each image was analysed 8 times for every number of points, with the points being re-randomised at every re-analysis. Mean and SD percentage cover of the benthic groups were calculated over the 8 values of cover per number of points, and the coefficient of variation (SD / mean) was calculated as a measure of the precision of the number of points in consistently quantifying the benthic groups. Low numbers of points corresponded to a high coefficient of variation, and the method gained precision with an increasing number of points. It was determined that for more than 80 points per $25 \times 25 \mathrm{~cm}$ there was no clear improvement in the coefficient of variation for most benthic groups (Fig. A3.1). Therefore, I chose to use 80 randomly assigned points per image, corresponding to 1280 points $\mathrm{m}^{-2}$, equal to 1 point 7.81 $\mathrm{cm}^{-2}$ on average. Consisting of 16 pictures per quadrat, 5 quadrats per site, and 3 sites sampled for 7 years, the entire data set comprised 134,400 points.

Table 4.1. Groups of benthic organisms or substrata used in the percent cover analysis.

\begin{tabular}{|l|l|}
\hline Benthic category & \multicolumn{1}{c|}{ Category description } \\
\hline \multirow{3}{*}{ Hard corals } & $\begin{array}{l}\text { Reef-building corals. Includes all scleractinian corals and also } \\
\text { stony hydrocoral Millepora sp. and octocoral Heliopora sp. } \\
\text { (not common in the quadrats). Most commonly encrusting } \\
\text { morphology. }\end{array}$ \\
\hline Sponges & $\begin{array}{l}\text { Any sponge taxon. Most commonly small-bodied encrusting } \\
\text { patches. See Chapter } 3 \text { for a list of the most common taxa } \\
\text { identified. }\end{array}$ \\
\hline
\end{tabular}




\begin{tabular}{|c|c|}
\hline Benthic category & Category description \\
\hline Algae & $\begin{array}{l}\text { - Fleshy and calcified macroalgae (not common on this reef at } \\
\text { the depth where the quadrats are, mostly Halimeda sp. and Padina } \\
\text { sp.). } \\
\text { - } \quad \text { Filamentous turfing algae (unidentified spp.). }\end{array}$ \\
\hline $\mathrm{CCA}$ & Crustose coralline algae (unidentified spp.). \\
\hline Other invertebrates & $\begin{array}{l}\text { - Octocorals, most commonly Clavularia sp., Sarcophyton sp., } \\
\text { whip corals. } \\
\text { - } \quad \text { Ascidians, most commonly Didemnum sp. and Polycarpa sp. } \\
\text { - } \quad \text { Bryozoans, hydrocorals, clams. Not commonly encountered } \\
\text { compared to other categories. }\end{array}$ \\
\hline Free space & $\begin{array}{l}\text { - Dead coral with corallites still visible, not yet overgrown. } \\
\text { - Bare rock or dead coral with corallites no longer visible. Not } \\
\text { overgrown. } \\
\text { - Fragments of dead coral skeleton not covered by other } \\
\text { organisms (coral rubble). Not common at this site as the reef is a } \\
\text { vertical wall and corals are mostly encrusting. }\end{array}$ \\
\hline Sand/silt & $\begin{array}{l}\text { - Sandy areas, limited to rare small ledges due to the steep } \\
\text { incline of the reef. } \\
\text { - Settled sediment that fully covers the underlying } \\
\text { (undiscernible) substrate. }\end{array}$ \\
\hline Undetermined & $\begin{array}{l}\text { - Live organisms that are visible in the image (i.e. not shaded, } \\
\text { blurred or covered by the quadrat) but are not identifiable as } \\
\text { any other category. }\end{array}$ \\
\hline Quadrat / shade & $\begin{array}{l}\text { - Used for points that could not be assigned to any benthic } \\
\text { category because they had fallen onto the quadrat frame, or } \\
\text { into a shaded area. Not used for calculating percentage cover. }\end{array}$ \\
\hline
\end{tabular}




\subsubsection{Decadal changes of benthic cover}

Changes in percentage cover of the live benthic taxonomic groups between sites and over time were analysed with linear mixed-effects models with normally distributed errors and random intercepts (LMMs, $R$ package nlme, Pinheiro et al. 2018). LMMs were chosen for our analyses because these models account for some properties of the data that would violate the assumption of more simple regression models, like repeated measurements and betweenquadrat heteroscedasticity. Mixed models were built with number of years after the first census and site as fixed effects, and with quadrat as a random effect. Quadrat was used as a random effect to account for the quadrat-related variation in the data. A continuous autocorrelation was fitted to the models to account for the temporal structure in the data, with correlation strength declining for years further apart from each other. Candidate models with different combinations of fixed effects (year and site) were fitted with maximum likelihood, and the most appropriate model was chosen by backwards step-wise selection with Likelihood Ratio Tests (LRT). A constant variance-covariance structure with as many levels as the number of quadrats, and with each level allowing for quadrat-specific variance (see Pinheiro et al. 2018) was then fitted to the best model. This variance structure accounted for non-homogeneous variance between quadrats. The final model was re-fitted with restricted maximum likelihood. For the fixed effects that were found significant, post-hoc pairwise tests with Bonferroni correction were applied to identify differences between sites ( $R$ package emmeans, Lenth 2019). Goodness of fit, normality and homoscedasticity of the errors were checked for all models by inspecting plots of the normalised residuals and the quantile-quantile plots (Fig. A3.2).

Previous studies that modelled percentage cover of benthic organisms as a dependent variable have relied on transformations like the arcsine or the isometric log-ratio, to handle the compositional nature of cover data that violates the assumption of equal variance of model errors (see models in Cooper et al. 2015, Gross et al. 2015). Because separate models were constructed for each benthic taxonomic group, thus controlling for between-group heteroscedasticity, and because fitting a variance structure to the models accounted for quadratspecific variance, the dependent variable was not transformed. Popular transformations like the arcsine are often difficult to interpret (Warton and Hui, 2011). 


\subsubsection{Spatial and temporal variation of community structure}

Because the most common benthic competitors at this location were hard corals, sponges and algae, the relative proportions of these three benthic taxonomic groups were visualised with ternary plots (R package ggtern, Hamilton 2017). Ternary plots are used to represent points as described by three coordinates, each representing one variable. The coordinates of points on a ternary plot must always add up to a fixed value (see Hamilton 2017). Therefore, percent cover data of corals, sponges and algae were transformed into ternary coordinates, in such way that the sum of the three groups was 100 . Ternary plots were used to evaluate whether the system was in a state of coral, sponge, or algal dominance, because these three were the three most abundant benthic competitors.

Changes in community structure across the three sites and over time were examined with coupled constrained and unconstrained ordination methods (Anderson and Willis 2003; $R$ package vegan, Oksanen et al. 2019). First, a Bray-Curtis similarity matrix (see Chapter 3) was derived from percentage cover data from all samples, i.e. each quadrat at each time point. Community structure was visualised with nonmetric Multidimensional Scaling (nMDS) in two dimensions (stress $=0.07$ ). The amount of variation explained by time (year) and space (site) was then quantified with a permutational MANOVA (Anderson 2001) with 9999 permutations. A Canonical Analysis of Principal coordinates (CAP, Anderson and Willis 2003) was performed to visualise the contribution of the benthic groups to samples from different sites and different years. See Methods section 3.2.5 in Chapter 3 for details on the multivariate statistics used in this chapter.

\subsubsection{Interannual changes of benthic cover of live benthic taxonomic groups}

Interannual changes in percentage cover of live benthic taxonomic groups were expressed as the $\log$-ratio $\varphi=\log \left(\mathrm{C}_{1} / \mathrm{C}_{0}\right)$, where $\mathrm{C}_{0}$ is percentage cover of group $X$ in year $y$ and $\mathrm{C}_{1}$ in year $y+1$. Log-ratio was used to allow for interannual changes of the same magnitude but of opposite sign to be represented as a signed deviation from zero. For example, suppose that group $X$ in year $y$ has $10 \%$ cover. A two-fold increase (doubling) between $y$ and $y+1$ would result in $20 \%$ cover in year $y+1$, and into $\mathrm{C}_{1} / \mathrm{C}_{0}=20 / 10=2$, whereas a two-fold decrease (halving) to $5 \%$ cover, and into $\mathrm{C}_{1} / \mathrm{C}_{0}=5 / 10=0.5 . \log (2)$ and $\log (0.5)$ have the same value (0.69) and opposite sign. Significant differences in average interannual variability between benthic groups were identified with a Kruskal-Wallis test, followed by post-hoc Dunn's test with Šidák correction for multiple comparisons to identify pairwise differences ( $R$ package dunn.test, Dinno 2017). 
For this test, the absolute value of the $\log$-ratio $|\varphi|$ was used to express interannual variability in such a way that the larger the interannual change the larger $|\varphi|$, and the smaller the change, the closer $|\varphi|$ is to zero. Not using the absolute values transformation would have centred the mean $\varphi$ for all benthic groups around zero (see Fig. 5), making differences in interannual variability undetectable by statistical tests comparing mean ranks, like the Kruskal-Wallis test.

\subsubsection{Lagged effects of free space on benthic cover}

To investigate the effects of free colonisable space on the abundance and growth of benthic organisms, the lagged effect of the amount of bare substrate available on the reef in year $y$ on the percentage cover of benthic taxonomic groups in year $y+1$ was investigated with LMMs. Models were built with percentage cover of bare substrate at time $y$ and site as fixed effects, and unique quadrat as a random effect. Model building, selection and validation were carried out as described above. In addition, because many benthic organisms have the potential to outcompete (or settle onto) filamentous turf algae and CCA, we re-ran these models three more times using as fixed effects site and: 1) the sum of bare substrate and turf algae cover; 2) the sum of bare substrate and CCA cover; and 3) the sum of bare substrate, turf, and CCA cover. These alternative models have the purpose of allowing the investigation of lagged relationships (lag of 1 yr) between substrate covered in generally weaker algal competitors and other benthic organisms.

All statistical analyses were performed in $R$ (version 3.5.3, R Core Team 2019), and all plots were created with the $R$ package ggplot 2 (Wickham 2009).

\subsection{Results}

\subsubsection{Temporal changes in percentage cover}

Percentage cover of benthic organisms at this reef was generally stable over the study period (Fig. 4.2). Hard corals were the most abundant benthic organism on the Buoy 3 wall, with a mean $( \pm \mathrm{SE})$ percentage cover of $42.69 \pm 1.86 \%$. The second most abundant benthic group on average was CCA $(23.38 \pm 0.98 \%)$, followed by sponges $(9.17 \pm 0.37 \%)$, algae $(8.53 \pm .0 .38)$, and other invertebrates $(8.32 \pm 0.75 \%)$. There was on average $4.66 \pm 0.27 \%$ of bare substrate open for settlement or growth of benthic organisms, and $3.22 \pm 0.21$ of sand and silt combined. Cover of undetermined organisms was $<0.05 \%$ on average and this group was not considered for further analysis. 
A

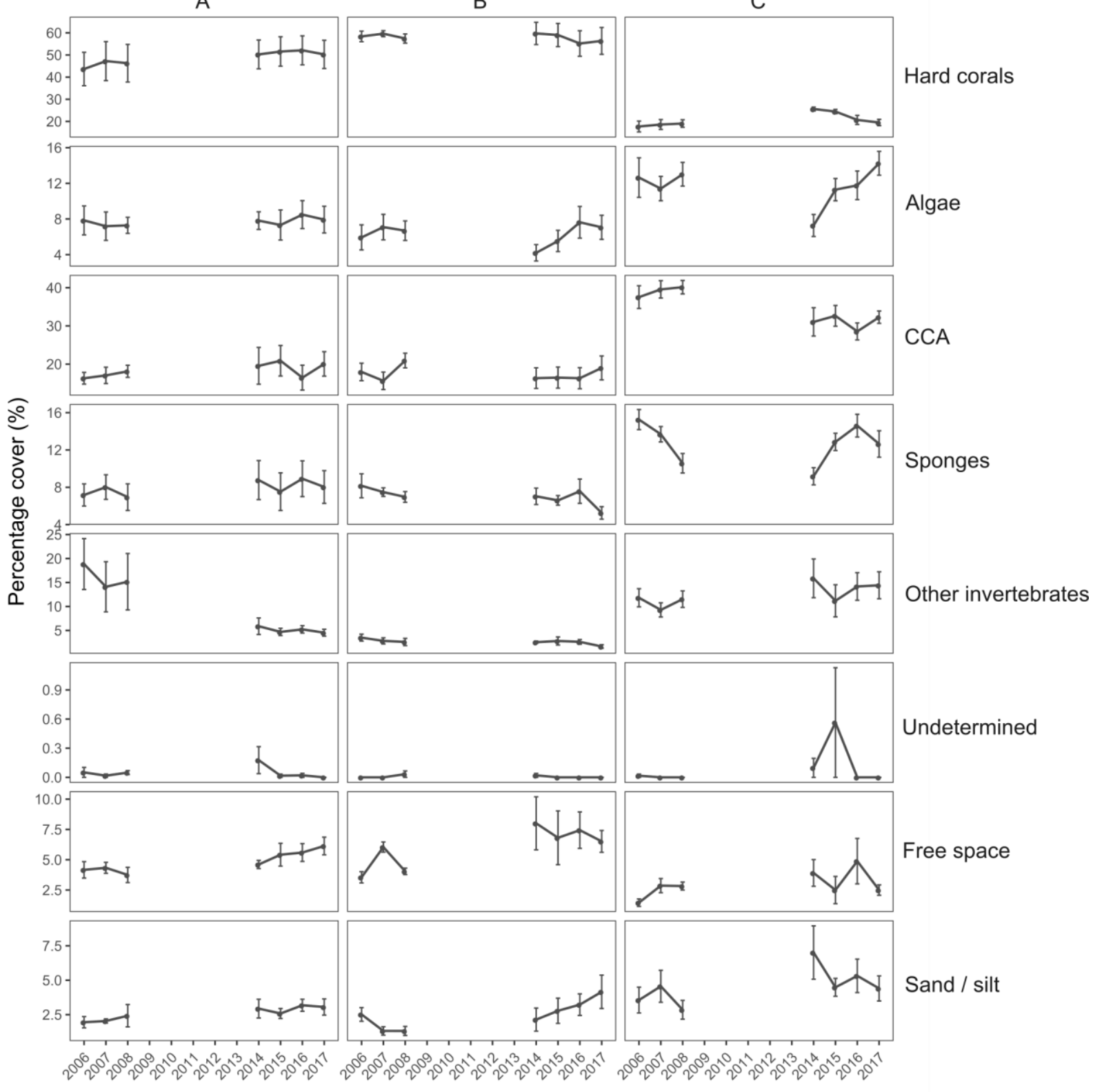

Figure 4.2. Mean percentage cover of benthic organisms at the Buoy 3 reef. Different columns (A, B, C) indicate the three sites. Vertical bars represent the standard error of the mean. Sites $A$ and $B$ are placed on a vertical wall, whereas Site $C$ is a steep incline (approx. $80^{\circ}$ incline). Note the different scales for different benthic categories.

Mixed models analysis of spatial differences and long-term changes showed limited temporal variation in percentage cover of live benthic taxonomic groups. Hard corals, sponges, and algae showed no significant long-term cover changes, but all three varied between sites (see Table 4.2 for complete LMMs results). Overall, percentage cover of benthic organisms at site $\mathrm{C}$ (the slightly inclined site) were different from sites A and B (the vertical wall sites). Namely, at site C, coral cover was lower than at sites A and B $(P=0.005$ and $P<0.001$ respectively), whereas 
sponge cover was higher $(P=0.014$ and $P=0.006$ respectively). Algal cover was also higher at site $\mathrm{C}$ than at site $\mathrm{B}(P=0.019)$. Coralline algae and other benthic invertebrates had sitedependent long-term dynamics. Coralline algae declined over time at site C (LMM-derived mean effect $-0.78,95 \%$ CIs [-1.27, -0.29]), whereas other organisms declined over time at site A, where soft corals had high abundance in one of the quadrats at the beginning of the study period (LMM-derived mean effect $-0.61,95 \%$ CIs [-1.10, -0.11]). Both CCA and other invertebrates showed no temporal trend at the other two sites. Overall, no group showed longterm variations that were consistent across space, showing that there is no evidence of overall change in dominance of benthic groups at this site.

Table 4.2. Results of the linear mixed-effects models (LMMs) showing the effects of site and year on percentage cover of the benthic taxonomic groups. Coefficient estimates, their standard error (SE), model degrees of freedom (DF), t-values, and p-values $(P)$ are reported for each fixed effect ( $\mathrm{P}$ values in bold are significant). Note that, for each model, the estimated Intercept refers to site $\mathrm{A}$, and the estimates for site $\mathrm{B}$ and site $\mathrm{C}$ indicate the difference between the Intercept at the respective sites and the intercept at site A. Similarly, the effect of year refers to site A, the estimates for the effect of year at site B and site C (indicated as [Year]:[Site]), indicate effect differences between the respective sites and site A.

\begin{tabular}{|c|c|c|c|c|c|c|}
\hline $\begin{array}{l}\text { Dependent variable } \\
\text { (\% cover) }\end{array}$ & Fixed effect & Estimate & SE & DF & t-value & $P$ \\
\hline \multirow[t]{3}{*}{ Coral } & Intercept & 46.983 & 4.755 & 89 & 9.881 & $<0.001$ \\
\hline & Site B & 10.953 & 6.806 & 12 & 1.609 & 0.134 \\
\hline & Site C & -27.046 & 6.681 & 12 & -4.048 & 0.002 \\
\hline \multirow[t]{3}{*}{ Sponges } & Intercept & 7.602 & 0.938 & 89 & 8.100 & $<0.001$ \\
\hline & Site B & -0.751 & 1.335 & 12 & -0.563 & 0.584 \\
\hline & Site C & 4.967 & 1.437 & 12 & 3.455 & 0.005 \\
\hline \multirow[t]{3}{*}{ Algae } & Intercept & 7.696 & 1.107 & 89 & 6.954 & $<0.001$ \\
\hline & Site B & -1.712 & 1.572 & 12 & -1.089 & 0.297 \\
\hline & Site C & 3.797 & 1.665 & 12 & 2.280 & 0.042 \\
\hline \multirow[t]{4}{*}{ CCA } & Intercept & 17.685 & 2.257 & 86 & 7.834 & $<0.001$ \\
\hline & Year & -0.022 & 0.164 & 86 & -0.133 & 0.895 \\
\hline & Site B & 1.345 & 3.181 & 12 & 0.423 & 0.680 \\
\hline & Site C & 22.017 & 3.293 & 12 & 6.687 & $<0.001$ \\
\hline
\end{tabular}




\begin{tabular}{|c|c|c|c|c|c|c|}
\hline $\begin{array}{l}\text { Dependent variable } \\
\text { (\% cover) }\end{array}$ & Fixed effect & Estimate & SE & DF & t-value & $P$ \\
\hline & $\begin{array}{l}\text { [Year]:[Site } \\
\text { B] }\end{array}$ & -0.161 & 0.236 & 86 & -0.682 & 0.497 \\
\hline & $\begin{array}{l}\text { [Year]:[Site } \\
\text { C] }\end{array}$ & -0.754 & 0.259 & 86 & -2.915 & 0.004 \\
\hline \multirow[t]{6}{*}{ Other Invertebrates } & Intercept & 9.385 & 1.705 & 86 & 5.503 & $<0.001$ \\
\hline & Year & -0.605 & 0.201 & 86 & -3.007 & 0.004 \\
\hline & Site B & -6.608 & 1.798 & 12 & -3.675 & 0.003 \\
\hline & Site C & 0.998 & 2.806 & 12 & 0.356 & 0.728 \\
\hline & $\begin{array}{l}\text { [Year]:[Site } \\
\text { B] }\end{array}$ & 0.534 & 0.212 & 86 & 2.516 & 0.014 \\
\hline & $\begin{array}{l}\text { [Year]:[Site } \\
\text { C] }\end{array}$ & 0.867 & 0.331 & 86 & 2.618 & 0.010 \\
\hline
\end{tabular}

\subsubsection{Community structure}
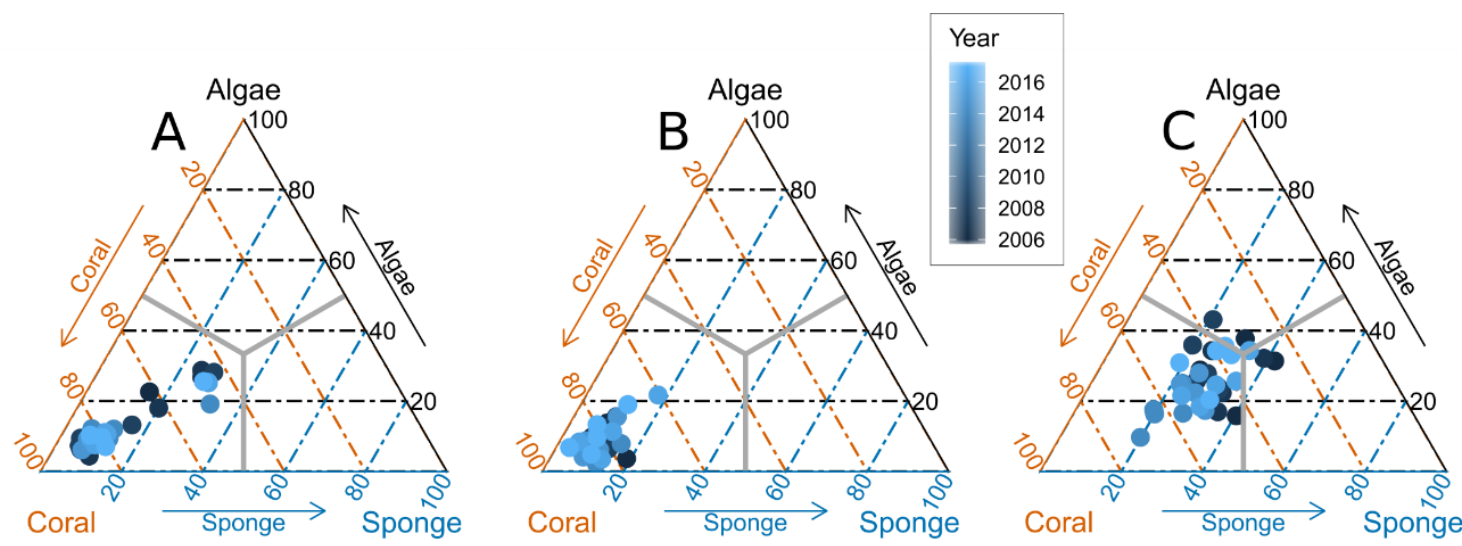

Figure 4.3. Ternary plots showing the relative proportions of the three main benthic competitors: hard corals, sponges and algae. Dots indicate single photoquadrats. The closer the dot is to a corner of the ternary plot, the more dominant the group in that corner is. Letters A, $\mathrm{B}$, and $\mathrm{C}$ indicate the three sites.

Scleractinian corals were the most abundant benthic group at sites A (mean and SE, $48.77 \pm$ $2.56 \%)$ and $\mathrm{B}(57.96 \pm 1.53 \%)$, but not at site C $(20.70 \pm 0.78 \%)$. Coralline algae were dominant at site $\mathrm{C}(34.60 \pm 1.14 \%)$, where cover of sponges and algae was also higher than at sites A and B (see Fig. A3.3 for estimates of mean effects of site and time on benthic cover). Considering the three main benthic competitors hard corals, algae and sponges, site $\mathrm{C}$ was the 
only site where hard corals were not the dominant benthic competitor at all times, with sponges or algae being more abundant than scleractinian corals (Fig. 4.3).

Like the abundance of the individual benthic categories, community structure of the reef was characterised by spatial heterogeneity and temporal stability. nMDS showed similarity between the composition of quadrats from the same site and from sites 1 and 2, highlighting the different composition of the benthos at site 3 (Fig. 4.4A). Samples from adjacent years, or even from the same year and different sites, did not show higher similarity than samples from years further apart. Permutational MANOVA results showed that site explained $60 \%$ of the variation in the community composition (Pseudo-F $=72.12, \mathrm{DF}=2, \mathrm{p}<0.001$ ), whereas year was not a significant predictor of community composition (Pseudo-F $=1.429, \mathrm{DF}=7, \mathrm{p}=0.182$ ).
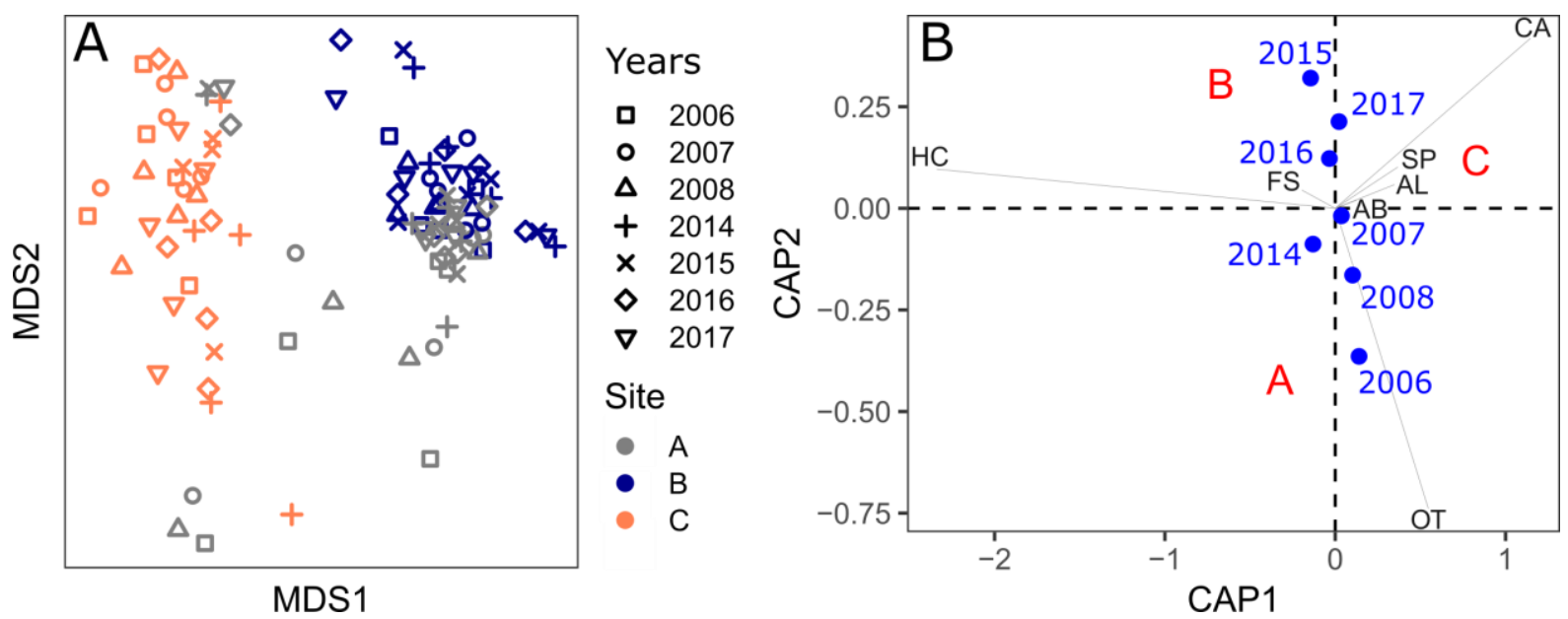

Figure 4.4. Spatial and temporal variation of community structure. A: Non-metric Multidimensional Scaling (nMDS) plot for the benthic community at the three sites in surveyed years between 2006 and 2017. Each dot corresponds to a quadrat. Symbols indicate the year, colours the site. B: Canonical Analysis of Principal coordinates (CAP) plot. Years and sites represent the centroid for each set of samples. HC: hard corals; SP: sponges; AL: algae; CA: CCAs; OT: other invertebrate; FS: free space; AB: abiotic (sand and silt). Category "Undetermined" not shown in CAP.

\subsubsection{Interannual changes of benthic cover of taxonomic groups}

Short-term temporal variability expressed as $\varphi=\log \left(\mathrm{C}_{1} / \mathrm{C}_{0}\right)$ was small for corals and CCA, and large for algae, sponges, and other invertebrates (Fig. 4.5). Significant differences in $|\varphi|$ were detected between live benthic groups at reef level (i.e. all sites pooled, $\chi^{2}=124.95, \mathrm{DF}=$ 
7, $P<0.001$, Kruskal-Wallis test). Pairwise comparisons between groups showed that, at reef level, interannual changes in coral cover were comparable to changes in CCA $(P=0.075)$, but were smaller than all other groups (algae, sponges, other invertebrates, all $P<0.001$ ). Shortterm temporal variability of sponge cover was comparable to that of algae $(P=0.991)$ and of other benthic invertebrates $(P=0.806)$.

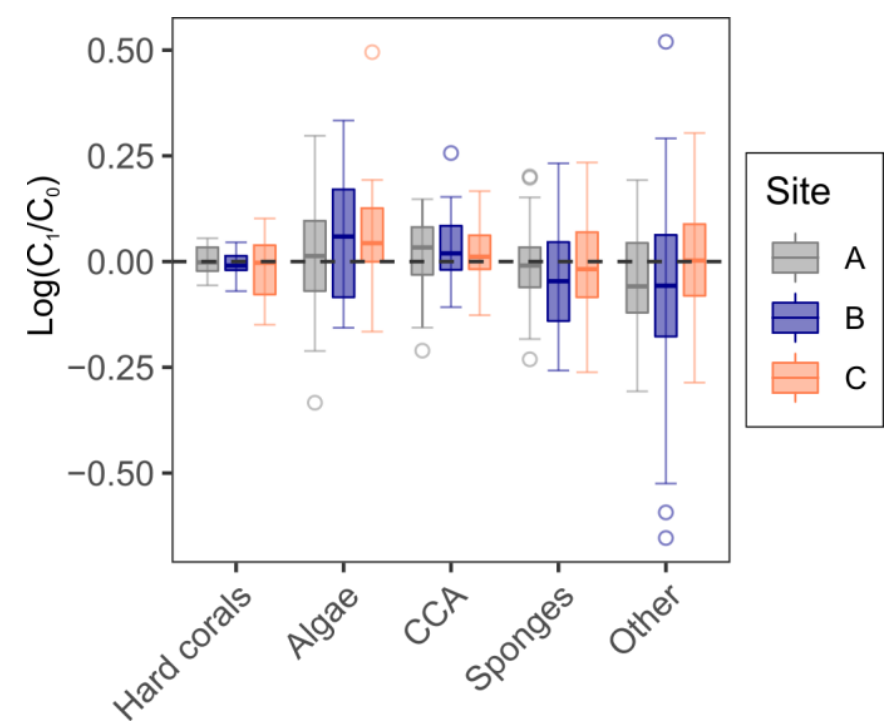

Figure 4.5. Box-plots showing the interannual variability of percentage cover of the benthic taxonomic groups, expressed as $\log (\varphi)=\log \left(\mathrm{C}_{1} / \mathrm{C}_{0}\right)$, where $\mathrm{C} 1$ is the cover of group $\mathrm{X}$ at time $y+1$ and $C_{0}$ is the cover of group $X$ in year y. Dashed horizontal line represents $\log \left(C_{1} / C_{0}\right)=0$, that is $\mathrm{C}_{1} / \mathrm{C}_{0}=1$, equal to no change between consecutive years. A large spread from the zeroline indicates high interannual variability for a group.

\subsubsection{Lagged effects of free space on benthic cover}

A positive lagged relationship was found between bare substrate and algal cover, uniformly across sites (mean effect $0.20,95 \%$ CI $[0.04,0.36], P=0.018$ ). When turf algae cover was added to bare substrate, a positive lagged relationship between these groups combined and CCA cover was detected (mean effect $0.60,95 \%$ CIs $[0.38,0.83], P<0.001$ ). A positive lagged relationship with sponge cover was also found at site A (mean effect $0.525,95 \%$ CIs [0.23, 0.82], $P<0.001$ ), but not at the other two sites. Similarly, when CCA cover was added to free space, a positive lagged relationship between this combined set and sponge cover was detected, but again only at site A (mean effect $0.43,95 \%$ CIs: [0.32, 0.55], $P<0.001$ ). Finally, when both turf algae and CCA were added to free space, there was once again a positive lagged relationship between the combined set and sponge cover at site A (mean effect $0.35,95 \%$ CIs: $[0.31,0.40], P<0.001)$, but not at the other two sites. 


\subsection{Discussion}

This study aimed to measure decadal and interannual variability in the percentage cover of benthic organisms on an Indonesian coral reef, and to investigate the effects of colonisable substrate available on the reef on benthic cover of organisms the following year. Percentage cover of all benthic organisms was relatively stable over time and showed no signs of longterm change. Similarly, community structure showed no significant changes over time, although differences in community structure between site $\mathrm{C}$ and the other 2 sites were detected. Sponge and turf algae showed larger interannual changes than hard corals and CCA. A positive lagged relationship between bare substrate and turf algae was detected. Furthermore, when percentage cover of turf algae and/or CCA were added to bare substrate and considered as colonisable substrate, a positive lagged relationship colonisable substrate and sponge cover was detected, but only at one of the sites, indicating that this relationship was not universal. These results show that: coral cover has remained stable at this Indonesian reef wall over the last decade; turf algae and sponges have the potential to fluctuate rapidly; of the benthic organisms, only turf algae had a direct benefit from free space becoming available on the reef.

\subsubsection{Long-term stability of benthic cover}

This coral-dominated Indonesian reef showed limited temporal variation between 2006 and 2017. Mean hard coral cover at reef level $(42.69 \pm 1.86 \%)$ was comparable to values reported by previous studies from the Wakatobi at a similar depth on the slope of non-degraded reefs (e.g. Bell and Smith 2004, Powell et al. 2014, Marlow et al. 2018a). For Buoy 3 (my study site), Powell et al. (2014) reported a $31 \pm 17 \%$ coral cover at a depth of $10 \mathrm{~m}$ in 2010.

Fleshy macroalgae were virtually absent from this reef, with the mean cover for this group never exceeding $0.05 \%$. Moreover, all observed algae were filamentous turf. Macroalgae in the central Indo-Pacific are often found in limited abundance on reef crests and slopes (e.g. Sawall et al. 2013, Plass-Johnson et al. 2016), and their abundance declined steadily across the Wakatobi between 2002 and 2007 (McMellor and Smith 2010) and until 2011 (Curtis-Quick 2013). McMellor and Smith (2010) reported mean historical levels of macroalgal cover in the Park of $\sim 23 \%$ before the decline. In contrast, macroalgal cover was always negligible in my data.

Although sponges were never the dominant benthic organism at Buoy 3, they were on average the third most abundant benthic group in terms of coverage $(9.17 \pm 0.37 \%)$, after hard corals and CCA. Only a few studies have measured percentage cover of sponges from Indo- 
Pacific coral reefs. Sponge cover from sites near Buoy 3 on Hoga Island at similar depth (10 m) were reported to have been between 10\% (Bell et al. 2018c) and 29\% (Bell and Smith 2004). Other records from the Indo-Pacific show variable sponge percentage cover (average of 5.37\% at $10 \mathrm{~m}$ in NE Kalimantan, de Voogd et al. 2009; 29\% at $5 \mathrm{~m}$ in East Timor, Farnham and Bell 2018). The relatively high abundance and diversity of sponges at this reef (Chapter 3, Rovellini et al. 2019) highlights their ecological importance.

Earlier studies carried out at a number of sites around Hoga Island have reported that coral cover declined between the early 2000s and 2012 (Curtis-Quick 2013, Powell et al. 2014, Gouraguine et al. 2019). In contrast with these other Wakatobi sites and with other Indo-Pacific reefs (Bruno and Selig 2007), no overall decline of coral cover was observed at Buoy 3 over the study period. My results are consistent with the findings of McMellor and Smith (2010), who reported that no decline in coral cover occurred at the reef wall off Hoga Island between 2002 and 2007, whereas corals had uniformly declined across other sites in the Park with a range of local-scale conditions. The stability of hard corals at this reef may be due to local characteristics of the coral assemblage and of the reef aspect. Hard corals in the Wakatobi are subjected to a range of stressors, including: increased sea water temperature linked to bleaching events (Wilson et al. 2012), increased sedimentation associated with decreased growth rates (Crabbe and Smith 2005), and destructive fishing methods (Clifton et al. 2010). However, corals at Buoy 3 were predominantly encrusting (see also description in Powell et al. 2015), and encrusting corals were the least affected coral growth form by past bleaching events that occurred in the region (Wilson et al. 2012). Furthermore, the accumulation of sediments on the reef wall at Buoy 3 is also lower than at nearby degraded reefs (Bell and Smith 2004, Marlow et al. 2018c), due to its vertical aspect (Bell and Barnes 2000a, b).

An increase in the percentage cover of the phototrophic sponge Lamellodysidea herbacea was reported between the early 2000s and 2015 for a highly-sedimented reef in the area (Bell and Smith, 2004, Powell et al. 2014, Biggerstaff et al. 2017b), but this sponge was not a dominant taxon in the sponge assemblage at Buoy 3 (Chapter 3, Rovellini et al. 2019). Sponge cover at Buoy 3 did not show an overall increase over time like it has at the degraded reefs in this region. Consistent with this result, sponge abundance (in number of patches per $\mathrm{m}^{-2}$ ) at this site, although highly dynamic, has also not changed significantly between 2005 and 2017 (Chapter 3, Rovellini et al. 2019). 


\subsubsection{Spatial and temporal variation in community structure.}

No temporal changes in community structure were detected at Buoy 3. Temporal stability of the community structure was consistent with the long-term stability of percent cover of taxonomic categories. The observed lack of long-term temporal changes in the composition of the benthos may be due to stable environmental conditions over the study period. For example, sea surface temperature and temperature anomalies in the Wakatobi had no detectable effect on sponge abundance and interannual growth, possibly due to the stability of temperature at this location (Chapter 3, Rovellini et al. 2019). However, spatial differences in the abundance of benthic organisms and in the community structure were detected. Site C generally had lower coral cover than sites A and B, and also had a different community structure. This difference is likely attributed to the slight inclination of the reef wall at site $\mathrm{C}$, as opposed to the vertical surface of sites A and B. Different inclination may result in different exposure to light and sedimentation, factors that can have an important impact on sponges and other benthic organisms (Cheshire and Wilkinson 1991, Carballo 2006, Bell et al. 2015b, McGrath et al. 2017). Furthermore, the vertical aspect of the reef at this location may be advantageous for corals. Hard corals settlement and survival has been found to be higher on vertical than on horizontal surfaces (Birkeland 1977, Rogers et al. 1984). The vertical aspect of this reef may thus have supported a stable coral assemblage over time, leaving limited scope for other benthic organisms to increase in their abundance.

\subsubsection{Interannual changes in benthic cover}

Interannual changes in percentage cover varied among the benthic taxonomic categories, with little interannual variation of hard corals and CCA, and larger variability for sponges, turf algae and other benthic invertebrates. Recent coral reef monitoring in the Spermonde Archipelago (South-West Sulawesi) over a 3-year period showed limited interannual changes in benthic cover of hard corals, sponges, other benthic invertebrates and macroalgae, but some degree of temporal variability in cover of CCA and turf algae (Teichberg et al. 2018). Low interannual variability of Indo-Pacific coral assemblages has also been reported from New Caledonia (Adjeroud et al. 2019, but see Adjeroud et al. 2002 for evidence of pronounced interannual variability in coral cover in response to natural disturbance in French Polynesia).

There is evidence of short-term temporal variability of sponge abundance and cover from sites within the Wakatobi. Biggerstaff et al. (2017b) reported a doubling in area covered by $L$. herbacea at Sampela over a period of 18 months. Moreover, while changes in sponge 
abundance expressed as number of sponge patches may not always correspond to changes in sponge percentage cover (Bell et al. 2017), mean sponge abundance at the Buoy 3 reef has showed large interannual variability, changing by as much as 40\% between 2014 and 2015 (Chapter 3, Rovellini et al. 2019). The interannual variability of sponge percentage cover was similar to that of algae and larger than that of hard corals (Fig. 4.5), which highlights that sponges at this site are highly dynamic on short time scales, while showing overall decadal stability.

\subsubsection{Comparing temporal changes in sponge abundance and sponge percentage cover}

The potential for high temporal variability in sponge abundance and diversity suggests that sponge-dominated reefs may be highly dynamic systems (Chapter 3, Rovellini et al. 2019). However, sponge percentage cover, whilst also displaying large inter-annual variability, did not show the same temporal dynamics as sponge abundance at the same reef.

While previous studies have reported a correlation between sponge abundance and percentage cover $\left(\mathrm{R}^{2}=0.46, P<0.001\right.$, Bell and Smith 2004), these two measures of sponge abundance may also be decoupled. Abrupt declines in sponge percentage cover may be due to mortality one or few large-bodied sponges, which may have limited impact on sponge numbers. Vice versa, large fluctuations in the number of sponge patches may not correspond to equal changes in sponge percentage cover if the sponges that are fluctuating in number are: (1) very small in size; and/or (2) cryptic cavity dwellers that are not well-captured by percentage cover data derived by photoquadrats. The sponge assemblage described in Chapter 3 was largely comprised of high-density, small encrusting sponge patches of the genera Protosuberites, Pericharax, and Sycon. Fluctuations in the abundance of these small-bodied sponges were responsible for the largest variations in sponge diversity, but did not correspond to equally large fluctuations in sponge percentage cover. In situ counts of number of sponge patches is a quick and non-destructive method for measuring sponge abundance, and it also allows measuring species diversity because it captures all sponges in the assemblage. However, fusion and fragmentation of sponges may confound the temporal development of sponge assemblages if in situ counts are the only measure of sponge abundance utilised (Bell et al. 2017). Furthermore, sponge counts may not convey the importance of sponges in the coral reef community compared with other benthic groups. Measuring the proportion of the reef area is occupied by sponges is useful to estimate the ecological importance of sponges in the benthic community. However, sponges frequently settle or grow over neighbouring benthic organisms 
(Rützler 1970, Whalan et al. 2012, Ribeiro et al. 2018), and percentage cover is not suited to quantify epibiosis. Furthermore, quantifying sponge biodiversity by measuring species-specific percentage cover in a sponge assemblage comprised mostly by small-bodied species is not precise, because pictures often fail to capture cryptic sponges.

Many biological and environmental controls may contribute to the decoupling of sponge abundance and percentage cover. Loss of sponge tissue to predation (Hoppe 1988) or diseases (Webster 2007) may cause the death or size reduction of some larger sponge patches that contribute to percentage cover. While mortality of shrinkage of larger sponges may not correspond to a decline in sponge abundance, they can lead to a decline in percentage cover. Conversely, a lack of temporal changes in percent cover may be due to biological controls and environmental conditions that limit sponge tissue growth. For example, macroalgae can competitively inhibit sponge growth on coral reefs (Easson et al. 2014, González-Rivero et al. 2016). Furthermore, slow and irregular growth has been observed in sponges exposed to environmental disturbances (Reiswig 1973) or recovering from an injury (Henry and Hart 2005). Due to the many factors that can control sponge percentage cover, it is important to complement this information with other measures of sponge abundance, like number of sponge patches.

Both sponge counts and sponge percentage cover have advantages and limitations when describing sponge abundance and biodiversity. If combined, these two measures can quantify: (1) metrics for sponge biodiversity, derived from the number of sponge patches for each species; and (2) abundance of sponges compared to other benthic organisms, expressed as percentage cover. Moreover, both in situ counts and percentage cover are non-destructive and easily collected measures. Accurate measures of sponge volume or biomass are often unpractical to attain and may require the destruction of the sponge (Bell et al. 2017), although sponge volume may be measured with photogrammetric techniques for some sponge morphologies like barrel sponges (McGrath et al. 2018). Discrepancies between temporal changes of sponge cover and sponge abundance at this Indonesian reef reported in this thesis highlight the importance of relying on multiple measures of sponge abundance, when possible (Bell et al. 2017).

\subsubsection{Effect of free space on benthic cover}

Occasional mortality of coral colonies, sponge patches, or other benthic organisms was apparent from our photoquadrats, although the causes of such mortality were not assessed. 
Upon death of a colony or individual, free space would become available for other organisms to expand, or for recruits to settle on. When bare substrate becomes available on coral reefs, it is rapidly covered by a film of bacteria or opportunistic microalgae (e.g. Crisp and Ryland 1960), which is then colonised by other benthic organisms (reviewed in Qian et al. 2007). Turf algae colonise bare rock after massive coral mortality events (reviewed in O'Brien and Scheibling 2018). Experiments on the early succession of benthic colonisers on recruitment tiles in the Indo-Pacific showed that filamentous turf algae are present on immersed surfaces as soon as 2 weeks after deployment (Bailey-Brock 1987), and that turf algae occupy up to $50 \%$ of free space 4 months after deployment (Plass-Johnson et al. 2016). A settlement study from Caribbean reefs shows that, while CCA and turf algae are the first group to colonise clean artificial substrate, encrusting invertebrates (mostly sponges) replace the algal groups by overgrowing thin algal crusts and small recruits of other organisms (Arnold and Steneck 2011). These authors also reported cover of invertebrate crusts off over $20 \%$ after one year from the deployment of the artificial substrate. In the present study, instead, only turf algae showed a positive relationship between bare substrate and percentage cover at the following census. This suggests that opportunistic algae promptly occupied the bare space that had become available on the reef, and that no other benthic organism had a direct and immediate benefit from the opening of free space.

Many benthic organisms can expand their margins or settle onto substrates other than barren rock. Studies from the Great Barrier Reef that have shown the importance of free space on coral recruitment and settlement of benthic invertebrates (Tanner et al. 1996, Connell et al. 1997). These studies have considered CCA and turf algae equivalent to free space, because truly empty space is available on the reef for a limited time and corals are able to settle on CCA (e.g. Heyward and Negri 1999, Negri et al. 2001). Moreover, filamentous turf algae have no significant effects on the settlement success of coral recruits (O'Brien and Scheibling 2018). However, ecological interactions between sponges and these algal groups are poorly understood. It has been shown that the larval settlement of the two Great Barrier Reef sponges Coscinoderma matthewsi and Rhopaloeides odorabile is induced by CCA (Whalan et al. 2012), but it is unclear whether all sponges can settle on CCA, and how competitive interactions between sponges and CCA are resolved. Similarly, turf algae have been considered part of bare substrate in a study on bioeroding sponges in the Wakatobi, because these sponges advance into calcareous substrate overgrown by turf (Marlow et al 2018a). However, information on sponge-algae interactions on coral reefs exists almost exclusively for encounters between 
sponges (mostly bioeroding species) and fleshy macroalgae, and only from reefs in the Caribbean (López-Victoria et al. 2006, González-Rivero et al. 2012, Easson et al. 2014).

My results showed mixed support for benthic organisms relying on algal substrates for colonisation. In this study, sponges had a positive lagged relationship with the combination of bare rock and algal substrates (turf, CCA and the two combined), although this effect was detected only at one site (Site 1). Some sponge taxa formed close associations with turf algae at this reef (Fig. A3.4). Sponges often form associations with other benthic organisms, including coral polyps and algae (Ribeiro et al. 2018), other sponges (Rützler 1970, Sarà 1970), and octocorals (Mclean et al. 2015). Calcareous sponges in particular have been found to form associations with many benthic organisms (Ribeiro et al. 2018). Sponges at this site are mostly small encrusting species, and some very abundant taxa at this site are small calcareous sponges like Pericharax sp. and Sycon sp. (Chapter 3, Rovellini et al. 2019). The positive lagged relationship between bare rock combined with algal groups cover and sponge cover at site 1 suggested that some sponge taxa at this reef might recruit to areas of the reef that are already fouled by algae. However, because this relationship was not consistent across sites, further research is needed to elucidate relationships between early-settled filamentous algae and other benthic organisms at this reef.

CCA have been shown to be fast colonisers of bare substrate. Boschetti (2016) reported a CCA cover of $\sim 40 \%$ on settlement panels in the Wakatobi one year after deployment, followed by a decline during the second year. Similarly, Arnold and Steneck (2011) showed that CCA cover peaks at around one year after deployment, and then is overgrown by thicker invertebrate crusts. Conversely, in the present study bare substrate alone had no effect on CCA cover at the following census, and CCA had a positive lagged relationship with bare space and turf algae combined, uniformly across all sites. This suggests that CCA at this site are not limited by bare substrate and can overgrow algal patches.

No lagged relationship was found between colonisable substrate and coral cover. This result is expected, given that encrusting corals, dominant at this reef, generally grow slower than branching growth forms (e.g. Dullo 2005), and that the lag used in this analysis was $1 \mathrm{yr}$.

In summary, the lack of delayed correlation between bare substrate and sponge percentage cover at this site suggests that either: 1) sponges at this site are not limited by bare substrate on the reef; or 2) sponges indirectly benefit from the opening of free space not by promptly colonizing it but by overgrowing the filamentous algae that occupy it first. In this second case, 
turfing algae colonise the empty space first, being the dominant group after one year, and other groups, including sponges, overgrow them or form associations with them on longer time scales. Due to the size of our dataset, it was not possible to explore higher-order lag relationships (i.e. $>1 \mathrm{yr}$ ) between free space and benthic groups.

\subsubsection{Implications for Indo-Pacific reef dynamics}

There was no evidence that a change in cover of benthic organisms has occurred in the last 13 years on this Indo-Pacific reef. Temporal stability of percentage cover at this reef is in contrast with other studies from reefs in the same region, which showed a decline in coral cover across several sites in the Wakatobi (Gouraguine et al. 2019), or a decline in coral cover that corresponded to an increase in sponge abundance (Biggerstaff et al. 2017b). Care should be taken in generalizing the findings of this study to the wider Indo-Pacific. Severe loss of corals has been occurring on many other coral reefs in the region (Bruno and Selig 2007), and IndoPacific reefs are threatened by long-term environmental change and by anthropogenic pressure (Graham et al. 2015). The extreme rarity of macroalgae from this reef should also be considered in the context of the depth and position of the Buoy 3 site. While macroalgae on Indo-Pacific reefs are not as abundant as they are in the Caribbean (Bruno et al. 2009, Plass-Johnson et al. 2016), they can be more abundant than hard corals on in shallow, low-water quality sites (PlassJohnson et al. 2015, Teichberg et al. 2018). Further research is needed to elucidate why the coral decline reported from other sites in the Indo-Pacific was not observed at this reef.

The results of this study indicate that, when benthic organisms die and bare substrate becomes available on the reef, sponges and other invertebrates may not immediately benefit from it. Instead, filamentous turfing algae rapidly occupy bare substrate, and CCA and sponges may benefit from turf cover by overgrowing it. Although the reef wall at this site has not experienced the same decline in coral cover and habitat degradation that several neighbouring sites have shown in the past decades, increasing threats of local anthropogenic pressure and global environmental change may result in increased coral mortality in the future. If this happens, and as turf occupies the bare substrate left behind, further research is needed to understand how other groups overgrow turf algae. The lack of long-term increase in algal cover indicates that, while turfing algae are the first and most efficient coloniser of free substrate, they are outcompeted or overgrown by other benthic invertebrates at subsequent successional stages, or they form associations with them. 
Results from this chapter guided the incorporation of some aspects of the ecology of coral reef benthic organisms in the Atlantis model (Chapter 6). Specifically, I included in Atlantis the option to capture the effects of turf algae on the settlement and growth of sponges and corals (see Chapter 6). Percent cover of free space and turf algae had a lagged positive effect on sponge percent cover only at one site in the present study, and the cover of turf-forming algae generally has no significant effects on coral settlement and recruitment (O'Brien and Scheibling 2018). However, as shown in this chapter, turf is an abundant and fluctuating component of coral reefs, and it interacts with other benthic sessile organisms in complex, species-specific ways (reviewed in O'Brien and Scheibling 2018). Because of the lack of a universal relationship between turf algae and corals, or turf algae and sponges, Atlantis was modified to allow for context-specific definition of the effect that turf has on the growth of other benthic groups (see Chapter 6). In addition, knowledge of the interannual variability of algae, sponges and other benthic invertebrates gained in this study was also useful to qualitatively validate temporal dynamics of the benthic groups modelled in Atlantis during the process of model calibration (see Chapter 6). 
Chapter 5. Reduced small-scale structural complexity on sponge-dominated reefs 


\begin{abstract}
Corals are important for providing structural complexity on coral reefs, and a complex 3D framework offers habitat to a wide diversity of coral reef fauna. While corals are well-known ecosystem engineers, the potential for other organisms to contribute to coral reef structural complexity has received limited attention. Sponges are expected to be more tolerant than corals to ocean warming and acidification and may become dominant on some coral reefs in the future. To understand how sponges contribute to reef 3D structure, I used 3D photogrammetry to compare the habitat complexity of coral-dominated and sponge-dominated portions $\left(4 \mathrm{~m}^{2}\right.$ quadrats) of an Indonesian coral reef. Structural complexity was expressed as rugosity $(R$, denoting complexity of reef contours), vector dispersion $(1 / k$, denoting heterogeneity of the angles between reef surfaces) and fractal dimension ( $D_{1-5}$, denoting complexity at five different spatial scales between 120-1 cm), all derived from computer-generated 3D models of the quadrats. I found that, at high habitat complexity, coral-dominated and sponge-dominated quadrats were similar in terms of $R$ and $1 / k$. However, complexity of larger refuge spaces $D_{2}$ (30-60 cm refuge holes) was higher for sponge-dominated quadrats $(P=0.005)$, whereas complexity of small-scale refuge spaces $D_{5}(1-5 \mathrm{~cm}$ refuge holes) was higher for coraldominated quadrats $(P=0.001)$, suggesting that corals provide smaller refugia compared to sponges, largely due to branching coral colonies. For coral-dominated quadrats, overall 3D structure was explained by the cover of branching and massive corals $(P=0.001$ and $P=0.002$, respectively), and for sponge quadrats by the cover of barrel morphologies $(P=0.008)$. In both coral-dominated and sponge-dominated quadrats, relationships between specific morphologies and complexity metrics varied, but were weak overall, suggesting that the combination of many growth forms defines the structural properties of the reef. This study suggests that spongedominated reefs will probably lack small-scale spatial structure, with negative implications for reef fauna that depend on small refugia. Given the central role of small cryptobenthic fish in reef food webs, this may have negative implications for reef trophodynamics on spongedominated reefs.
\end{abstract}

\title{
5.1. Introduction
}

Three-dimensional structure is an important physical feature of terrestrial and marine ecosystems and is provided not only by the underlying geology, but also by biogenic matrices and by live organisms (Kleypas et al. 2001). Structural complexity provides the associated 
biota with the opportunity for niche partitioning (MacArthur and MacArthur 1961), refuge from predators (Hixon and Beets 1993, Steele 1999), shelter for larvae and recruits (Johnson 2007), larger areas available for habitation (Heck and Wetstone 1977), and it dissipates the mechanical stress caused by wind (Finnigan 2000) or waves (Monismith et al. 2015). Extensive research has shown that complex habitats are associated with high species richness and abundance across many ecosystems, including forests (MacArthur and MacArthur 1961, Tews et al. 2004), freshwater streams (O'Connor 1991), temperate intertidal reefs (Kelaher and Castilla 2005), the deep sea (Buhl-Mortensen et al. 2010), and coral reefs (Graham and Nash 2013). Because structural complexity has these important ecological roles and because habitatbuilding organisms contribute to structural complexity, it is important to understand how changes in the abundance of structure-building organisms affect the three-dimensional complexity of an ecosystem.

On coral reefs, scleractinian corals are considered the most important structure-building organisms (reviewed in Graham and Nash 2013), as they can build carbonate skeletons in complex three-dimensional growth forms. Coral-associated structural complexity is a strong predictor of fish abundance and diversity (e.g. Messmer et al. 2011, Darling et al. 2017) and of invertebrate diversity (Stella et al. 2011). Heterogeneous reef structure also hosts a greater variety of fish sizes (Nash et al. 2013) and improves resilience of associated fish assemblages after a disturbance (Emslie et al. 2014). Furthermore, a complex coral framework on fringing reefs offers protection from waves to inshore ecosystems (like reef lagoons and mangroves) and to inhabited coastlines (reviewed in Ferrario et al. 2014). Different coral species and growth forms contribute in different ways to structural complexity (Denis et al. 2017, Richardson et al. 2017), with structurally complex growth forms like tabular and branching colonies being keystone providers of structural complexity (e.g. Shima et al. 2008, Kerry and Bellwood 2015a, Ferrari et al. 2016). The critical importance of structural complexity for coral reef ecosystems has set the focus of recently proposed coral reef management strategies on framework-building corals (Darling et al. 2019).

The decline of corals due to global climate change and local-scale anthropogenic pressures (see Hoegh-Guldberg et al. 2007, Baird et al. 2013, Cheal et al. 2017, Hughes et al. 2017a, Brown and Hamilton 2018) has corresponded to a decrease in structural complexity on coral reefs (Wilson et al. 2006). Reef flattening associated with coral loss has been described across the world's oceans, including the Caribbean (Alvarez-Filip et al. 2009), the Indian Ocean (Graham et al. 2006, Elliott et al. 2018), the Indo-Pacific (Jones et al. 2004), the Great Barrier 
Reef (Emslie et al. 2014), and the Central Pacific (Magel et al. 2019). A reduction in coral reef structural complexity is predicted to result in the loss of habitat-associated reef fish (Holbrook et al. 2015, Newman et al. 2015), changes in fish assemblage composition (Messmer et al. 2011), reductions in fishery productivity (Rogers et al. 2014, 2018), and higher exposure of coastlines to the mechanical energy of waves (Harris et al. 2018).

Regardless of whether reef flattening has co-occurred with the loss of corals, corals have been declining globally. While regime shifts towards macroalgal-dominated states are widely documented (e.g. Mumby et al. 2007, Bruno et al. 2009), on some coral reefs other sessile invertebrates may become the dominant benthic organisms after hard corals declined (Norström et al. 2009). A decline in corals has corresponded to increased sponge abundance at some locations in the Caribbean (Diaz and Rützler 2001, McMurray et al. 2010), Brazil (Kelmo et al. 2013), the Indo-Pacific (Powell et al. 2014, Biggerstaff et al. 2017b) and the Central Pacific (Schils 2012, Knapp et al. 2013, 2016). Furthermore, experimental evidence indicates that some tropical sponge species, although vulnerable to increased temperature (Bennett et al. 2017, Ramsby et al. 2018), may be more tolerant than corals to ocean warming and acidification (e.g. Stubler et al. 2015, Bennett et al. 2018, see Chapter 1 for further references). As a result of the previously reported shifts towards sponge-dominated reef systems after coral mortality, and because of their resistance to climate change conditions, sponges have been proposed to be among the potential winners on future coral reefs (Bell et al. 2013, 2018b).

Sponges have many important functional roles on coral reefs (reviewed in Bell 2008), including removal of organic matter from the water column (de Goeij et al. 2008), hosting of microorganisms (Taylor et al. 2007), bioerosion of the reef framework (Schönberg et al. 2017), silicon cycling (Maldonado et al. 2005), competition for space (Wulff 2006a), and provision of food for predators (Pawlik et al. 2018). Furthermore, sponges can grow into complex threedimensional structures (Boury-Esnault and Rützler 1997), and thus they contribute to structural complexity of the seafloor, especially when they are present at high density and diversity (Maldonado et al. 2016). The importance of sponges as ecosystem engineers is highlighted by the many associations between sponges and other organisms, promoted by their external threedimensional framework and the internal system of aquiferous canals (see reviews in Wulff 2006a and Bell 2008). For example, Mediterranean sponges can host diverse endobiotic communities of invertebrates (e.g. Koukouras et al. 1996, Gerovasileiou et al. 2016, Papatheodoulou et al. 2019). Deep sea invertebrate fauna is also strongly associated with the presence of sponge beds of high structural complexity (Buhl-Mortensen et al. 2010), with 
sponges offering refuge from predators (Beazley et al. 2013) and substrate for growth of other sessile suspension feeders (Beaulieu 2001). Associations between fish diversity and deep-sea sponge grounds have also been reported (Kenchington et al. 2013), and temperate deep-water sponge habitats are used by juvenile fish to escape predation (Ryer et al. 2004, Scharf et al. 2006). Similarly, three-dimensionally complex sponge assemblages in estuarine ecosystems supports higher fish abundance and species richness (van Lier et al. 2017).

On coral reefs, sponges can provide refuge space for juvenile decapod crustaceans (Herrnkind et al. 1997), and they have been proposed as nursery grounds and alternative habitats for fish in degraded reef systems (Seemann et al. 2018). However, corals are still considered the most important contributors to reef structural complexity (Graham and Nash 2013), and despite the ecological importance of sponges on coral reefs and the potential for an increase in sponge abundance on future reefs, their contribution to reef structural complexity has not been investigated.

Sponges in the Indo-Pacific are an abundant and diverse component of coral reefs (e.g. Bell and Smith 2004, Chapter 1), and sponge assemblages in this region have high morphological diversity (Bell 2007, Hadi et al. 2015). Low-complexity, encrusting sponges have recently become dominant on some degraded reefs in this region (Biggerstaff et al. 2017b), and are generally abundant on reef flats and crests exposed to hydrodynamic turbulence (Bell and Smith 2004), but more complex and upright growth forms become more abundant with depth (Bell 2007, Bell et al. 2018c). Large growth forms like the barrel sponge Xestospongia sp. (McGrath et al. 2018) and the fan-shaped Ianthella sp. (Maldonado et al. 2016) are also present at high density at many sites in the Indo-Pacific, thus contributing to reef structure. If sponges do become more abundant on future coral reefs, it is important to understand whether they can provide a structural complexity comparable to that of corals (Chapter 2, Bell et al. 2018a), and which sponge growth forms contribute the most to the architecture of the reef.

An increasing number of methodologies for underwater 3D photogrammetry have recently been developed. These methods use computer-generated models of the three-dimensional structure of the reef at a range of spatial scales, from single corallites or coral colonies (Lavy et al. 2015, Gutierrez-Heredia et al. 2016), to quadrats (Young et al. 2017), transects (Burns et al. 2015, Leon et al. 2015), and even entire reef-scapes (Friedman et al. 2012, Ferrari et al. 2016). From these 3D models, it is then possible to measure variables that are difficult to derive in situ, but that provide useful information on reef structural complexity, such as fractal 
dimension (Bradbury and Reichelt 1983). Fractal dimension $(D)$ is a measure of threedimensional complexity at different spatial scales (for example $\mathrm{cm}$ to $\mathrm{m}$ ), and it can be used to compare irregularly shaped organisms or reef areas (Reichert et al. 2017), and to explore relationships between reef architecture and the associated fauna (e.g. González-Rivero et al. 2017, Hunt et al. 2019). Applications of underwater 3D photogrammetry focussing on structural complexity of coral reefs have so far only focused on corals (but see Lawler and Osborne 2008 for photogrammetric classification of temperate sponges).

In this chapter, I applied the underwater 3D photogrammetry technique proposed by Young et al. (2017) to characterise the structural properties of coral-dominated and sponge-dominated areas of an Indonesian coral reef, at an intermediate spatial scale ( $4 \mathrm{~m}^{2}$ quadrats). Reef structural complexity was expressed as rugosity $(R$, expressing complexity of the linear contour of the reef), vector dispersion (1/k, expressing heterogeneity of the angles between reef surfaces), and fractal dimension ( $D_{1-5}$, expressing reef structural complexity at 5 different spatial scales, from small refuge holes of $1-5 \mathrm{~cm}$ to larger crevices of $60-120 \mathrm{~cm}$ ). The aims of this chapter were: 1) to identify differences between 3D structure of coral-dominated and sponge-dominated reef patches at different spatial scales; 2) to understand which sponge growth forms contribute the most to overall structural complexity; and 3) to understand which sponge growth forms contribute to the different complexity metrics $\left(R, 1 / k\right.$ and $\left.D_{1-5}\right)$.

Characterizing the structural properties of sponge-dominated reefs and comparing such properties to those of coral reefs is a first necessary step to understand the role of sponges as providers of structural complexity on sponge-dominated reefs. One important follow-up question is whether sponge-provided structural complexity has the same ecological functions of coral-provided framework. These functions include the important one of creating habitat for other coral reef organisms, and it will be crucial to understand whether structurally complex sponges will be able to compensate for the loss of three-dimensional habitat caused by coral decline. The fish assemblage associated with coral-dominated and sponge-dominated reef areas rendered with 3D models was also recorded. Data on fish assemblages was collected outside the scope of this thesis (in collaboration with another student). However, the results of the analysis of the fish data will be published with the data reported in this chapter. 


\subsection{Materials and Methods}

\subsubsection{Study location and sampling design}

The location of this study was Hoga Island in the Wakatobi Marine Park (South-East Sulawesi, Indonesia, Fig. 5.1). Data was collected using SCUBA in August 2017 at two reefs, known locally as Ridge 1 (a sloping reef wall) and Outer Pinnacle (the top of a seamount near the main reef of Hoga Island). On the slopes of these two reefs combined, a total 32 quadrats $(2 \times 2 \mathrm{~m})$ were purposely positioned on portions of the reef where either hard corals or sponges were highly abundant. For each quadrat, $4 \times 1 \mathrm{~m}^{2}$ photoquadrats were taken for subsequent percentage cover quantification. Pictures were taken with a Canon PowerShot G12 (10.0 MP). Default settings were used (auto mode), and a camera white balance against a white slate was performed at the depth of each quadrat before shooting. Quadrats were placed at depths between 6 and $22 \mathrm{~m}$. Because this study focused on the inherent structural properties of the reef associated with the abundance of corals or sponges, regardless of relationships between benthic organisms and physical variables, site and depth were not relevant for the study design, and they were not considered in the analyses. Quadrats spanned a range of structural complexity, from low-complexity quadrats dominated by encrusting growth forms of either corals or sponges to high-complexity quadrats with high cover of branching corals or complex sponge morphologies. Each quadrat was filmed with a lawnmower pattern using an action camera (GoPro Hero 5 Black), with resolution set on 1080p and field of view set on "narrow" while hovering approximately $1 \mathrm{~m}$ above the substrate, following the method described by Young et al. (2017). These videos were used to construct 3D computer models of the reef quadrats (see below). 


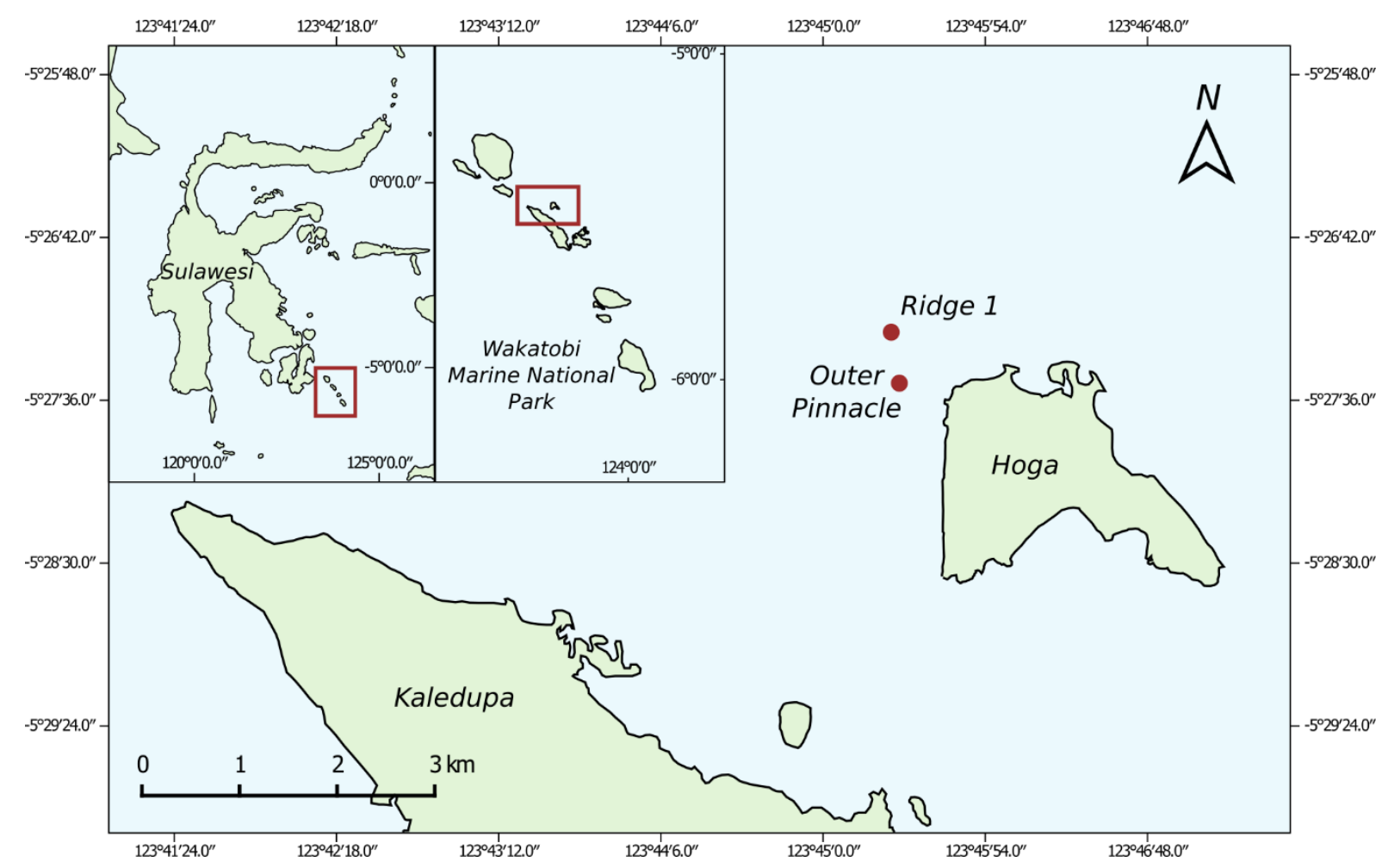

Figure 5.1. Study location on Hoga Island, Wakatobi Marine Park, SE Sulawesi (Indonesia). Red dots indicate the two reefs where the sampling occurred.

\subsubsection{Substrate characterization}

Percentage cover of benthic taxonomic groups, abiotic substrates and of common coral and sponge growth forms were measured with the software Coral Point Count with Excel extensions (CPCe, Kohler and Gill 2006), by overlaying 300 random points per photoquadrat (i.e. 1200 points per $4 \mathrm{~m}^{2}$ quadrat). Points were assigned to one of the following benthic groups: hard corals, sponges, crustose coralline algae (CCA), turf algae, macroalgae, soft corals, ascidians, other invertebrates, undetermined organism, dead coral, rock, rubble, sand, and silt. Furthermore, hard corals were assigned to one of seven morphologies: branching, table, foliose, massive, submassive, free-living (mushroom corals), and encrusting (Fig. 5.2A). Sponges were assigned to one of ten growth forms (derived from Boury-Esnault and Rützler 1997): branching, tubular, repent, foliose (fan-shaped), digitate, barrel, massive, cushion, globular and encrusting (Fig 5.2-B). While Bell (2007) reported 39 sponge morphologies on coral reefs off Hoga Island, the morphological categories used for sponges in the present study were less detailed, because: 1) subtle differences between growth forms are 
often difficult to determine from pictures; and 2) the goal of my morphological categories was to capture general three-dimensional properties of sponge fauna.

Based on percentage cover analysis, quadrats were categorised as either coral-dominated or sponge-dominated. Because there is no consensus in the literature regarding how benthic dominance should be defined (e.g. Bruno et al. 2009), a benthic group was considered dominant in a quadrat if it had the highest benthic cover of all benthic groups (including abiotic substrates) and it occupied at least $1 / 3$ of the reef area (>33.33\% cover).

\subsubsection{D photogrammetry of the reef and complexity metrics}

Three-dimensional computer models (Fig. 5.3) of the 32 quadrats $\left(4 \mathrm{~m}^{2}\right)$ were built following the protocol described by Young et al. (2017). Still frames were extracted from the videos of the quadrats ( 3 images per second) with the software Adapter (version 2.1.2.0, Macroplant LLC 2019) with quality set on "high". Still frames were loaded into Photoscan Standard (version 1.3.3, Agisoft LLC 2017) and rendered into a 3D model with the following steps (based on Young et al. 2017): (1) after automatic camera calibration carried out by Photoscan's proprietary algorithm, pictures were aligned with quality set on "highest" (key point limit = 40,000; tie point limit $=4,000)$; (2) a dense point cloud was built at "ultra high" quality, and extraneous points created with this process were manually removed from the models; (3) a mesh was built with maximum face counts set to 3,000,000; (4) and a texture was built with default settings. Following the guidelines presented by Young et al. (2017), quality of the models was visually assessed, and models were accepted if the scale bar represented by the quadrat's PVC frame was visible. Quality of the rendering of the quadrat's PVC frame was important because a minimum of 2 vertices of the PVC frame are used as Ground Control Points in subsequent analysis of the 3D models. Models were then exported and analysed with the 3D modelling software Rhinoceros 3D ("Rhino", version 5 SR14, Robert McNeel and Associates 2017). Python scripts developed by Young et al. (2017) were used in Rhino to calculate 7 measures of structural complexity: rugosity $(R)$, vector dispersion $(1 / k)$ and fractal dimension $(D)$ at five scales of refuge space complexity $\left(D_{1-5}\right)$. These metrics are defined in Young et al. (2017), and described briefly here. Rugosity $R$ is analogous to traditional chainand-tape measures (Risk 1972) of linear rugosity and ranges between 1 (flat surface) and 0 (complex surface - note the inverse scale). Vector dispersion $1 / k$ measures the heterogeneity of the angles formed by the reef surface, and ranges between 0 (flat surface) and 1 (complex surface). Fractal dimension $D$ is composed by 5 values $\left(D_{1-5}\right)$ and it measures structural 
complexity of the reef surface within pre-determined size categories. Values of $D_{1-5}$ correspond, respectively, to refuge holes with diameters of $120-60 \mathrm{~cm}, 60-30 \mathrm{~cm}, 30-15 \mathrm{~cm}$, $15-5 \mathrm{~cm}$, and 5-1 cm. Values of each $D$ metric range between 2 and 3, with larger values indicating higher complexity at that scale of refuge spaces.

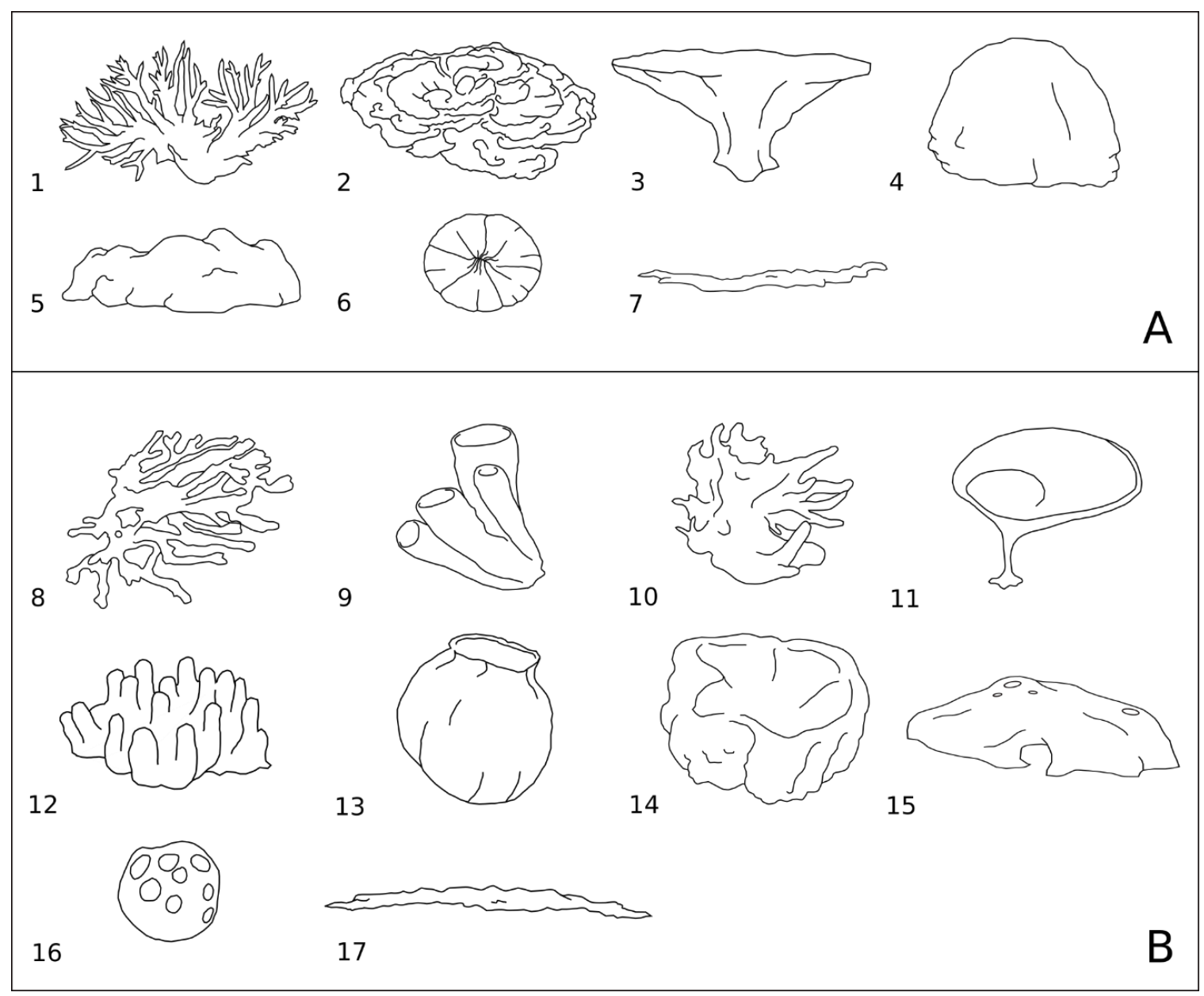

Figure 5.2. Most common coral (A) and sponge (B) growth forms found on Hoga Island. Corals: 1: branching; 2: foliose; 3: table; 4: massive; 5: submassive; 6: free-living (mushroom); 7: encrusting. Sponges: 8: branching; 9: tubular; 10: repent; 11: foliose (fanshaped); 12: digitate; 13: barrel; 14: massive; 15: cushion; 16: globular; 17: encrusting. 


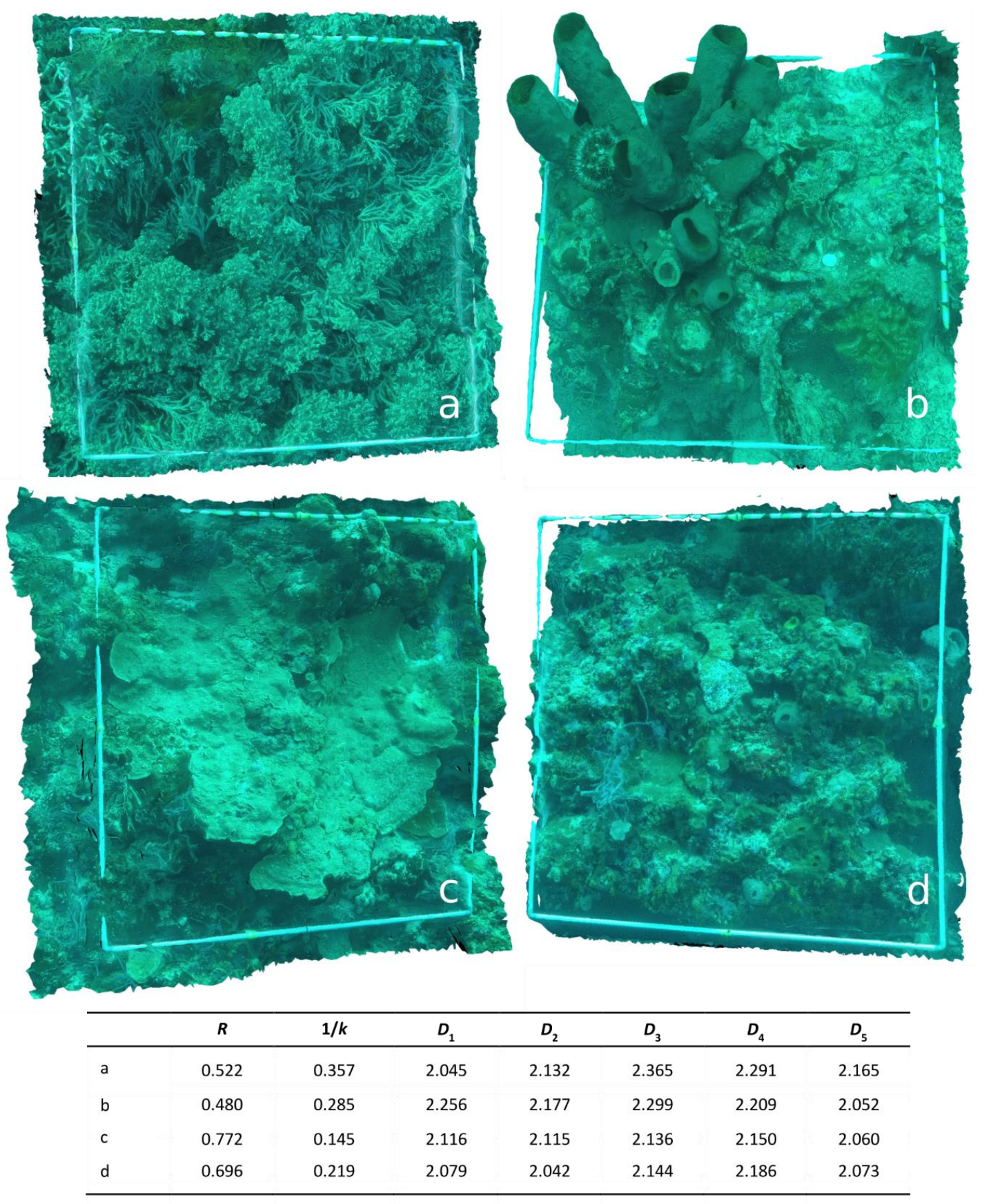

Figure 5.3. Examples of 3D models of $4 \mathrm{~m}^{2}$ quadrats, with table reporting the respective values for the 7 metrics of structural complexity. (a) High-complexity coral-dominated quadrat (Q06), dominated by branching corals and characterised by high heterogeneity of the surfaces $(1 / k)$ and intermediate- to small refuge space complexity $\left(D_{3-5}\right)$; (b) high-complexity spongedominated quadrat (Q02), with tubular sponges, characterised by high rugosity (low $R$ ) and large refuge space complexity $\left(D_{1}\right)$; (c) low-complexity coral-dominated quadrat (Q24), and (d) low-complexity sponge-dominated quadrat (Q14), both characterised by encrusting growth forms and comparatively low values for most metrics. 


\subsubsection{Structural properties of coral and sponge quadrats}

A matrix of the Euclidean distances between the quadrats was calculated based on the 7 standardised metrics of structural complexity. Euclidean distances were chosen because the target matrix was a set of continuous variables, and therefore a distance matrix was desirable over a similarity matrix for the subsequent analysis. Complexity metrics were standardised before calculating the Euclidean matrix to be on the same scale for the clustering method. Agglomerative hierarchical cluster analysis with Ward's method (Ward 1963, Murtagh and Legendre 2014) was then applied to this matrix to group the quadrats according to their overall structural complexity. Clustering-based grouping of the quadrats into high- and lowcomplexity was done in order to compare complexity metrics between coral-dominated and sponge-dominated quadrats that had similar structural properties (e.g. to compare complex coral-dominated quadrats with complex sponge-dominated quadrats). Ward's method, which works by minimising the total within-cluster variance (Ward 1963, Murtagh and Legendre 2014), was used because it allows the user to decide a priori the final number of clusters that the samples need to be grouped into ( 2 in this case: high-complexity and low-complexity quadrats for each dominant benthic organism).

Generalised Linear Models (GLMs) were used to determine whether dominant benthic organism (coral or sponge, as factor) and level of complexity (high or low, as factor, as determined by the clustering) determined differences in the 7 complexity metrics. The appropriate GLM was chosen depending on the distribution of the modelled variable, and goodness of fit was evaluated by inspecting model deviance residuals. $R$ and $1 / k$ (constrained between 0 and 1) were modelled with beta regression and a log link function (Ferrari and Cribari-Neto 2004, $R$ package betareg, Cribari-Neto and Zeileis 2010). Metrics $D_{1}, D_{2}$ and $D_{5}$ had a skewed right distribution, whereas $D_{3}$ and $D_{4}$ were normally distributed. Therefore, $D_{1}$, $D_{2}$, and $D_{5}$ were modelled with GLMs with gamma-distributed errors and a log link function, and $D_{3}$ and $D_{4}$ with GLMs of the Gaussian family and an identity link (equivalent to ordinary least squares regression). All models were built with dominant organism and level of complexity as predictors. When significant effects of the predictors were detected, post-hoc analyses with Bonferroni correction were applied to detect pairwise differences between coralsponge quadrats and between high-low complexity quadrats ( $R$ package emmeans, Lenth 2019). 


\subsubsection{Contribution of growth forms to overall 3D structure}

Constrained ordination methods were employed to determine whether percentage cover of the coral growth forms explained variation in the overall structural complexity of coraldominated quadrats (Anderson 2001, Anderson and Willis 2003; $R$ package vegan, Oksanen et al. 2019). First, an ANOVA-like permutation test (function anova.cca, Oksanen et al. 2019) was performed on the matrix of Euclidean distances (already derived for the hierarchical clustering, see above) to quantify how much variance in the overall 3D structure was explained by different coral growth forms. Redundancy Analysis was used to visualise how quadrats were organised in the multivariate space defined by the complexity metrics, and how cover of different morphologies was related with the quadrats and with the 7 complexity metrics. Redundancy Analysis is an ordination method used to tests for the effects of a set of explanatory variables (\% cover of morphologies of the benthic organisms) on a matrix of Euclidean distances (the matrix of the complexity metrics, see Legendre and Legendre 2012). The same analysis was performed on sponge-dominated quadrats to determine how different sponge growth forms contributed to overall structural complexity. Furthermore, the effects of coral growth forms on the 3D structure of sponge-dominated quadrats, and vice versa, were also determined with the same method, to assess whether benthic taxonomic groups other than the dominant ones significantly contributed to overall structural complexity in the quadrat.

\subsubsection{Contribution of growth form to specific complexity metrics}

To determine which growth forms were good predictors of which complexity metrics, variable selection was conducted by fitting GLMs with Least Absolute Shrinkage and Selection Operator (LASSO) regression ( $R$ package glmnet, Friedman et al. 2010). LASSO regression is a regularization method for fitting linear models and for variable selection, and it is robust to small sample size, (Friedman et al. 2010), even up to cases where the number of predictors equals the number of samples (Zou and Hastie 2005). While fitting a model, LASSO penalises the predictors that contribute the most to model variance by shrinking their coefficients to zero (Tibshirani 1996). The method achieves this shrinkage by deriving a penalization coefficient, $\lambda$, and by applying it to the coefficients of the predictors (the growth forms). Seven models per dominant benthic organism (coral or sponge) were built, one for each of the 7 complexity metrics, with coral and sponge growth forms as explanatory variables for coral and sponge quadrats, respectively. All 7 dependent variables were modelled with GLMs of Gaussian family (equivalent to ordinary least squares), even though not all response variables were unbound and normally distributed (see Section 2.4). For this step, this was considered an 
acceptable approximation because model fitting via LASSO regression had the purpose of variable selection, and model estimated coefficients were not used for further predictive modelling; therefore, ease of interpretation of the models was preferred over precision of the estimated coefficient. Furthermore, $R$ package glmnet recommends the use of GLMs of Gaussian family with identity link in case of continuous numeric response variable, like the 7 structural metrics (Friedman et al. 2010). Models were fitted with 10-fold cross-validation, which returned a value of $\lambda$ that allowed to shrink to zero the coefficients of the weak predictors while minimizing model uncertainty $\left(\lambda_{\min }\right)$. Because the folds for the cross-validation are randomly selected by the fitting algorithm, the cross-validation was iterated 100 times (i.e. with 100 random seeds). Larger numbers of random iterations were tested and returned the same results, therefore 100 random iterations were considered sufficient. Growth forms whose coefficients were not shrunk to zero in at least $90 \%$ of the random iterations of the crossvalidation procedure were considered good predictors for the complexity metrics. To control for the contribution of sponge growth forms to complexity metrics in coral quadrats, and vice versa, sponge morphologies were tested as predictors of complexity metrics in the coral quadrats, and vice versa with the LASSO method, following the same procedure as above.

All statistical analyses were performed in $R$ (version 3.5.3, R Core Team 2019), and all plots were created with the $R$ package ggplot 2 (Wickham 2009). 


\subsection{Results}

\subsubsection{Benthic cover and levels of structural complexity}

In 15 of the 32 quadrats, hard corals were the most abundant benthic group (mean and SE coral cover $59.22 \pm 3.65 \%$ ), whereas in the remaining 17 quadrats sponges were the most abundant benthic group (46.05 $\pm 1.40 \%$, Fig. 5.4).

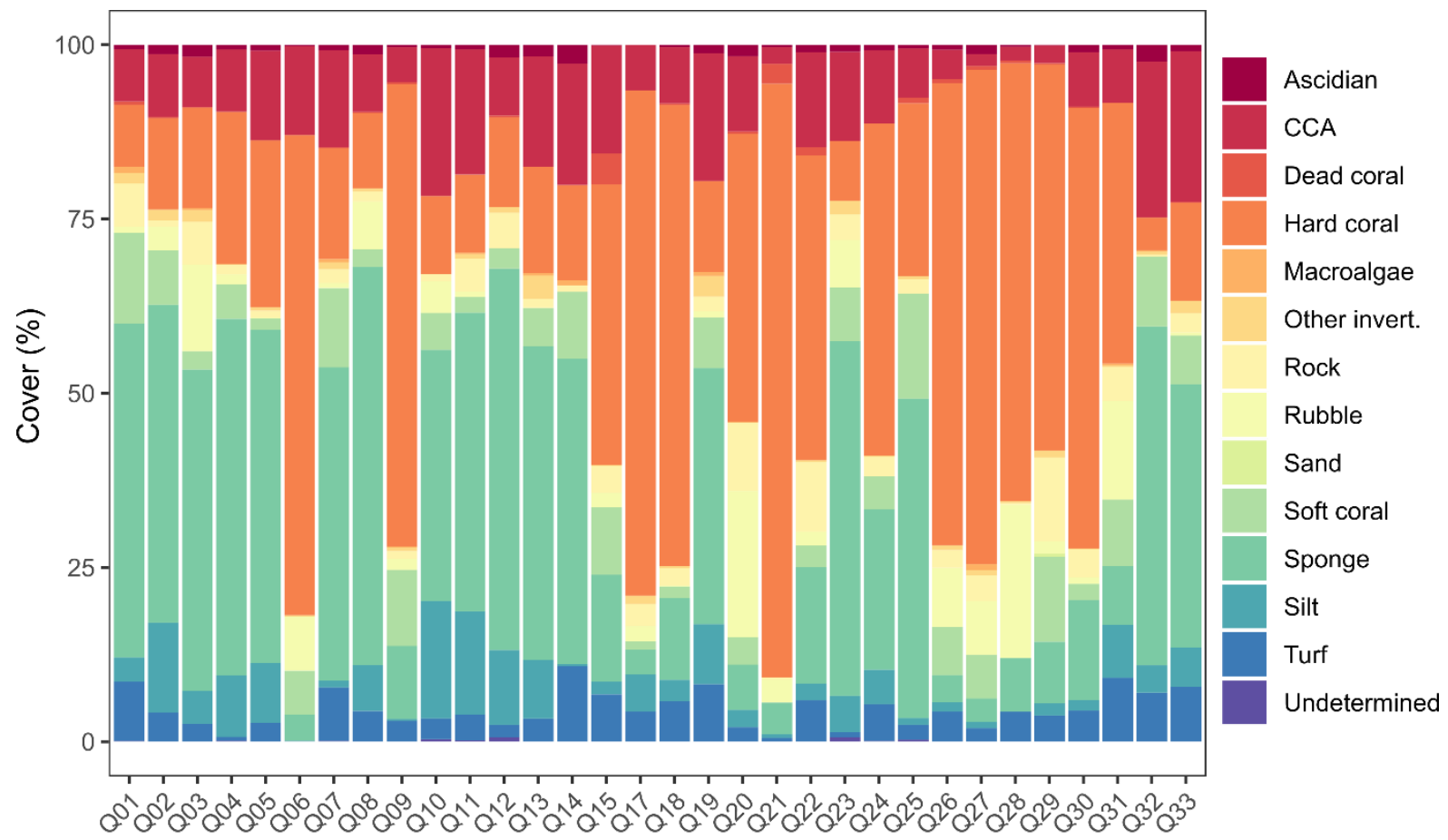

Figure 5.4. Percentage cover of benthic taxonomic groups and abiotic substrates in the $324 \mathrm{~m}^{2}$ quadrats.

Hierarchical clustering based on the 7 complexity metrics divided both coral-dominated and sponge-dominated quadrats into two complexity-based groups (Fig. 5.5). The separation of these two clusters for each dominant organism allowed the separation of quadrats into highand low-complexity quadrats, based on their structural properties. On average, coral quadrats identified as "high complexity" were characterised by branching (mean \pm SE $31.71 \pm 4.44 \%$ ), foliose $(10.01 \pm 3.27 \%)$, and massive corals $(6.86 \pm 1.54 \%)$, whereas low-complexity coral quadrats had on average high cover of encrusting (22.70 \pm 2.06$)$, submassive $(15.33 \pm 2.85 \%)$, and tabular (12.37 $\pm 2.23 \%$ ) coral growth forms (Fig. 5.6). Quadrats with high percentage cover of tabular coral were identified as low-complexity quadrats, indicating that the method could not capture the overhangs and the habitat provided by coral tables (see Discussion). On average, high-complexity sponge quadrats were characterised by encrusting $(16.81 \pm 0.42 \%)$, barrel $(9.99 \pm 1.51 \%)$, and tubular morphologies $(6.86 \pm 1.31 \%)$, whereas low-complexity 
sponge quadrats were, on average, characterised by encrusting $(19.39 \pm 0.71 \%)$, massive $(9.96$ $\pm 0.66 \%)$ and cushion $(6.15 \pm 0.44 \%)$ morphologies (Fig. 5.6). Encrusting sponges were the most abundant sponge growth form in both high- and low-complexity sponge quadrats. On average, bare rock and dead coral skeleton had low percentage cover $(3.26 \pm 0.52 \%$ and 0.42 $\pm 0.16 \%$, respectively).

A

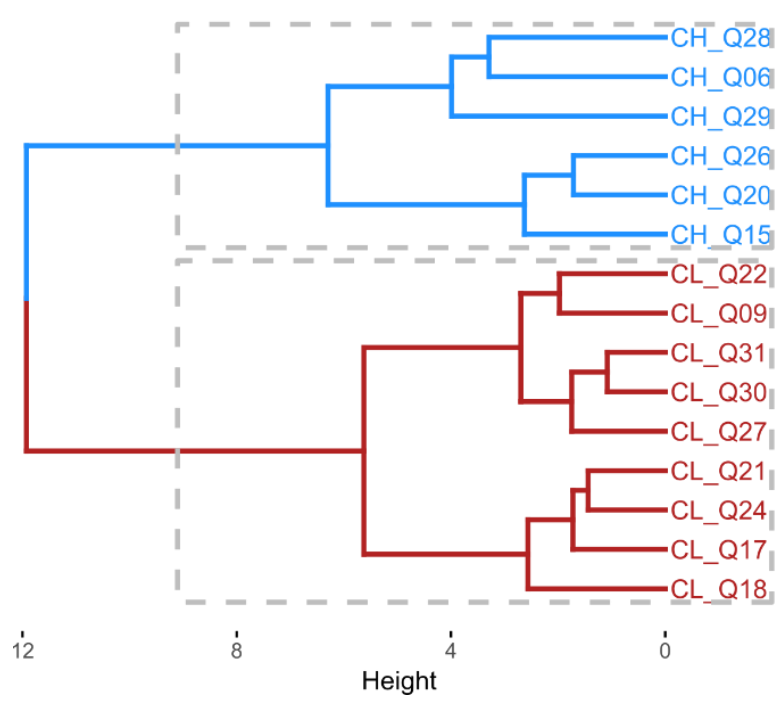

B

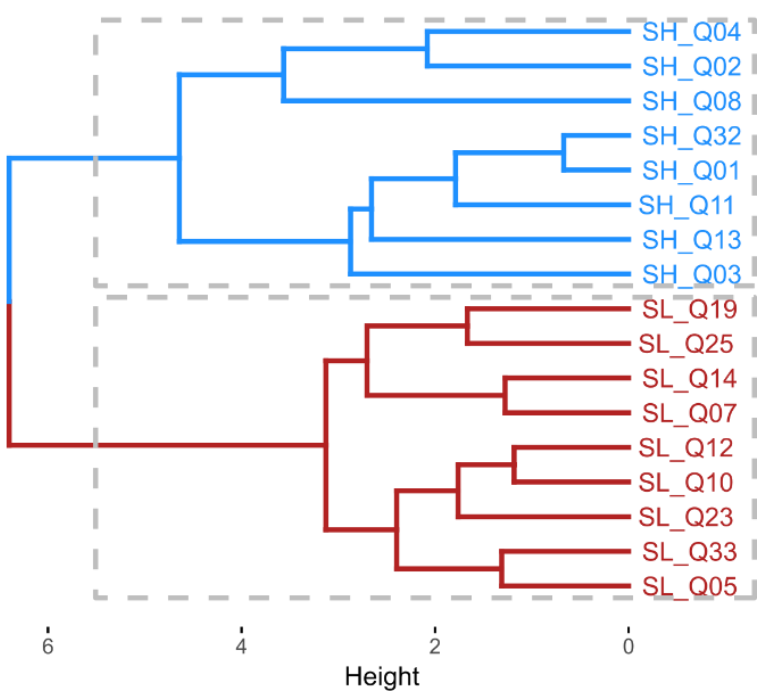

Figure 5.5. Hierarchical clustering based on the matrix of Euclidean distances calculated on the multivariate matrix of the 7 structural complexity metrics. A: coral-dominated quadrats. B: sponge-dominated quadrats. Blue quadrats are high-complexity, red quadrats are low complexity. Coral-dominated and sponge-dominated quadrats are denoted by the letters $\mathrm{C}$ and $\mathrm{S}$, respectively. High-complexity and low-complexity quadrats are denoted by the letters $\mathrm{H}$ and L, respectively. 

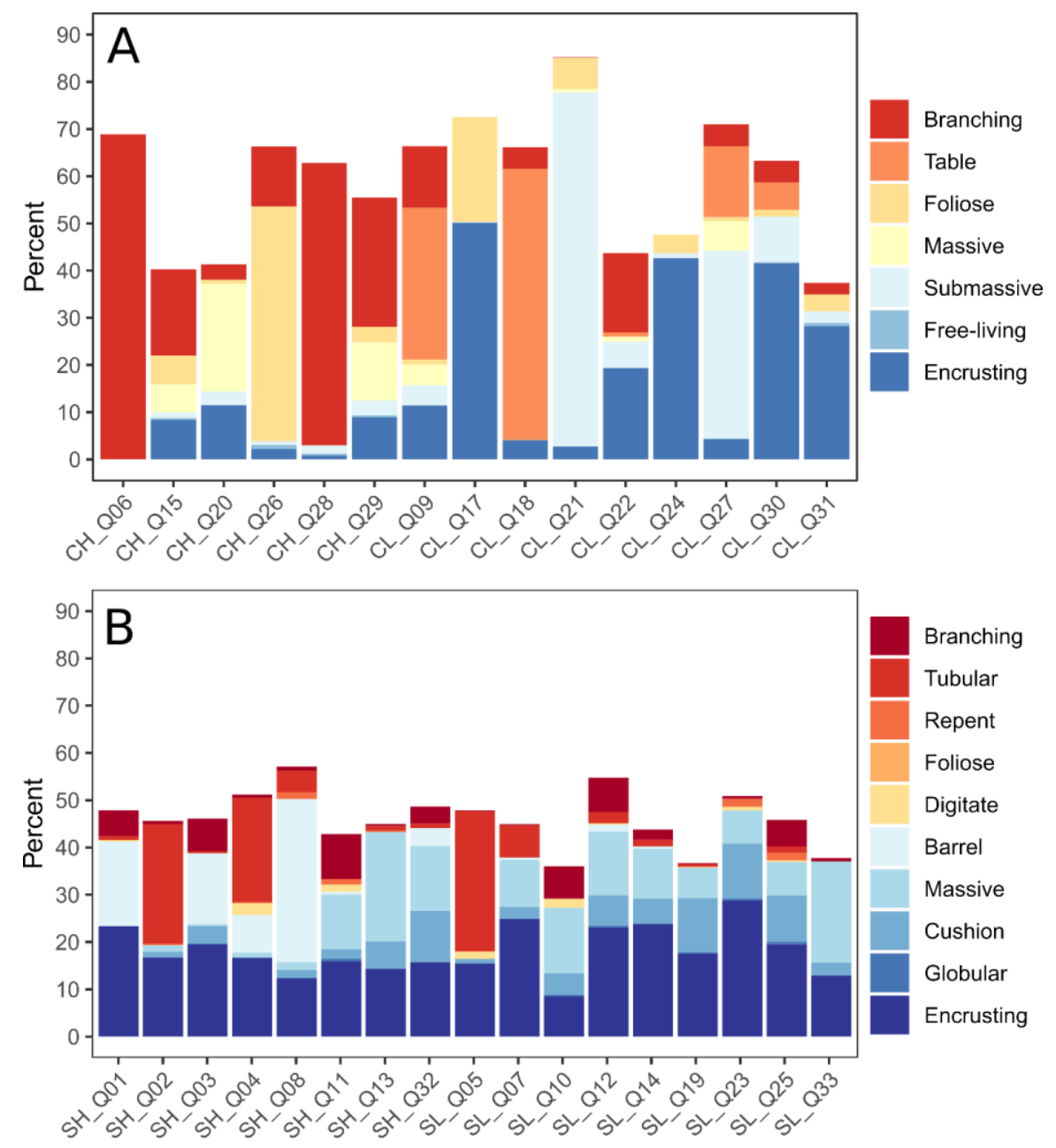

Figure 5.6. A: percentage cover of coral morphologies in coral-dominated quadrats; B: percentage cover of sponge morphologies in sponge-dominated quadrats.

\subsubsection{Structural properties of coral and sponge quadrats}

At low structural complexity, rugosity $R$ was lower (i.e. more complex surface) in spongedominated quadrats than in coral-dominated quadrats $(P=0.0011)$, but similar between coraldominated and sponge-dominated quadrats at high complexity $(P=0.8528)$. For both coral and sponge quadrats, $R$ decreased (i.e. rugosity increased) with complexity $(P<0.0001$ and $P=$ 0.0072 for corals and sponges respectively). Similarly, vector dispersion $1 / k$ (reflecting heterogeneity of the surfaces) at low complexity was higher for sponge quadrats than for coral quadrats $(P=0.0002)$ but similar between coral-dominated and sponge-dominated quadrats at high complexity, and increased with complexity for both coral and sponge quadrats $(P<$ 0.0001, $P=0.0494$ respectively). Fractal dimension metrics $D_{1}$ and $D_{2}$ that described large refuge spaces (120-60 cm and 60-30 cm, respectively) increased with complexity for sponge quadrats $\left(P=0.0039\right.$ and $P=0.0015$ for $D_{1}$ and $D_{2}$, respectively) but not for coral quadrats. 
Furthermore, at high complexity, $D_{2}$ was higher in sponge-dominated quadrats than in coraldominated quadrats $(P=0.0054)$. The opposite pattern was found for intermediate- and smallscale fractal dimension metrics $D_{3}, D_{4}$ and $D_{5}$ (indicating refuge holes of sizes $30-15 \mathrm{~cm}, 15-5$ $\mathrm{cm}, 5-1 \mathrm{~cm}$, respectively), which increased with complexity for coral quadrats (all $P<0.0001$ for $D_{3-5}$ ), but not for sponge quadrats. Importantly, $D_{5}$ at high complexity was higher in coral quadrats than in sponge quadrats $(P=0.0009)$. Significant differences between coral and sponge quadrats and between levels of complexity for all metrics are reported in Fig. 5.7.
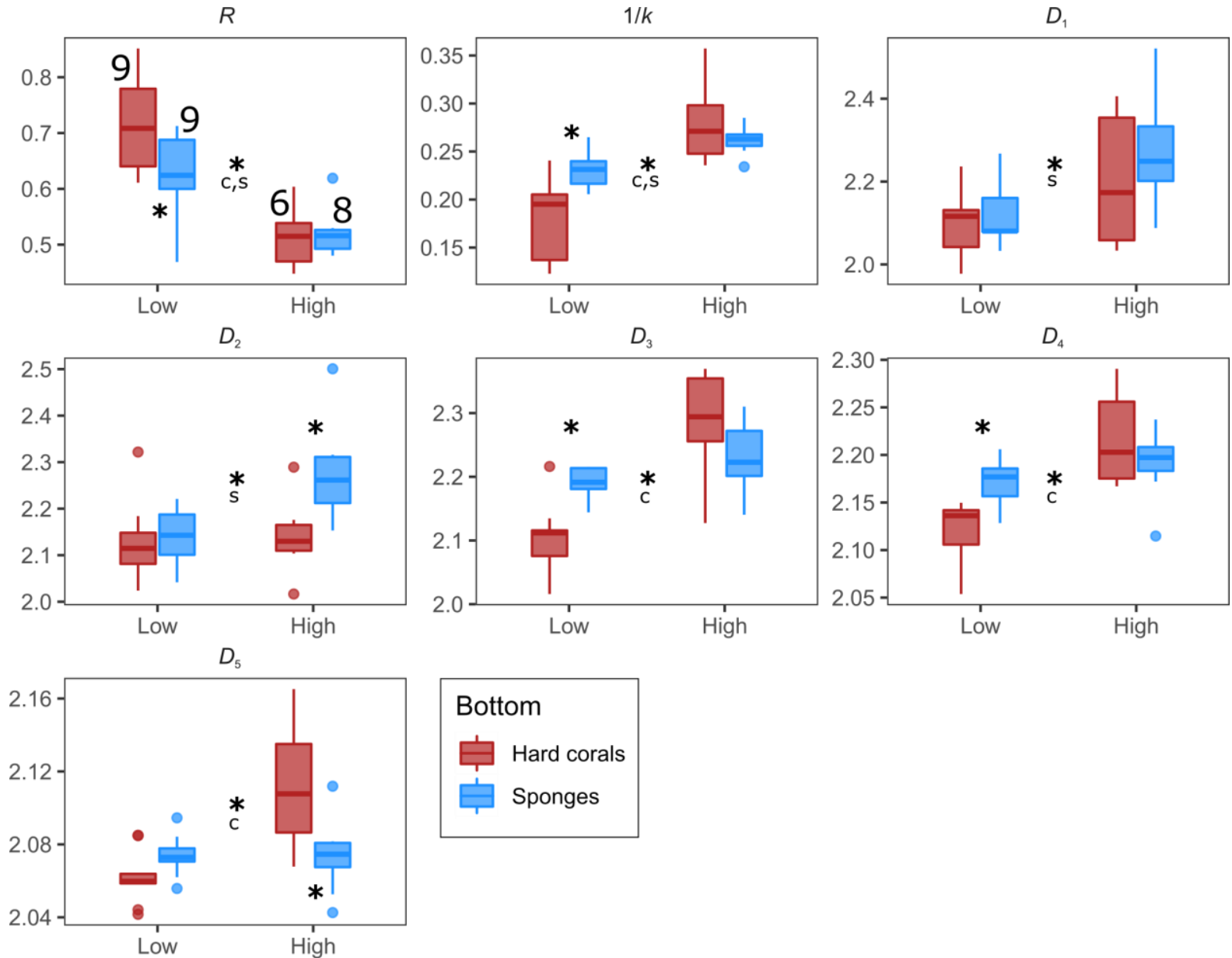

Complexity

Figure 5.7. Boxplots of the model-derived habitat complexity metrics for coral (red) and sponge (blue) quadrats, at low and high levels of structural complexity. Note the different scales on the y axis for different metrics. Asterisks indicate significant differences reported by the Generalised Linear Models, either between coral and sponge quadrats (asterisk in between boxes) or between levels of complexity for the group indicated by the letter (asterisk in the centre of the plot, $\mathrm{c}=$ corals, $\mathrm{s}=$ sponges). $R=$ rugosity (note the inverse scale), $1 / k=$ vector dispersion, $D_{1-5}=$ fractal dimension. Numbers in the first panel represent sample size $(N)$. 


\subsubsection{Contribution of growth forms to overall 3D structure}

Redundancy analysis based on overall structural complexity (i.e. Euclidean matrix calculated based on the 7 structural metrics) showed that the cover of encrusting, sub-massive and tabular corals was associated with low-complexity coral quadrats, whereas cover of branching and massive corals was associated with high-complexity quadrats (Fig. 5.8-A). The ANOVA-like permutation test showed that variance in the overall structural complexity of coral-dominated quadrats was explained by the cover of branching $(48.13 \%$, pseudo- $F=20.64, P=0.001)$ and massive coral growth forms $(17.63 \%$, pseudo- $F=7.56, P=0.002)$. For sponge-dominated quadrats, barrel, tubular and digitate growth forms were associated with high-complexity quadrats, whereas encrusting, cushion, massive and globular growth forms were associated with low-complexity quadrats (Fig. 5.8-B). However, ANOVA-like permutation tests showed that only barrel growth forms explained a significant amount of variation in the overall structural complexity of sponge-dominated quadrats $(20.29 \%$, pseudo- $F=3.53, P=0.008)$. Importantly, no sponge morphology explained significant variation in the overall structural complexity of coral-dominated quadrats, and vice-versa (results not shown).
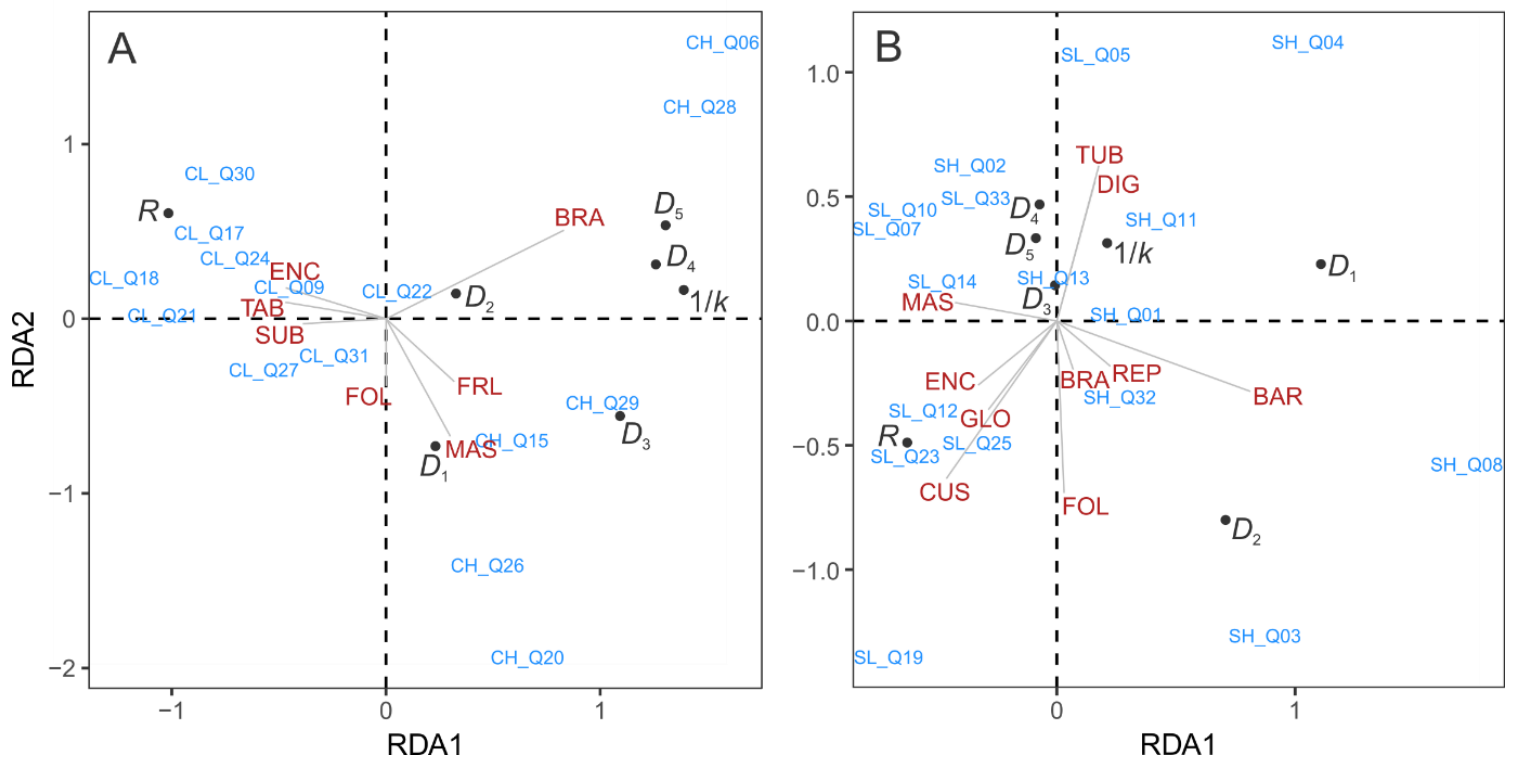

Figure 5.8. Redundancy Analysis plots that show which growth forms correlate with high- and low-complexity quadrats, and how quadrats are distributed in the multivariate space defined by the complexity metrics. A: coral-dominated quadrats and coral morphologies. B: spongedominated quadrats and sponge morphologies. $R=$ rugosity (note the inverse scale), $1 / k=$ vector dispersion, $D_{1-5}=$ fractal dimension, $\mathrm{BRA}=$ branching, $\mathrm{TAB}=$ table, $\mathrm{FOL}=$ foliose, MAS = massive, $\mathrm{SUB}=$ sub-massive, FRL = free-living, ENC = encrusting, $\mathrm{TUB}=$ tubular, $\mathrm{REP}=$ repent, $\mathrm{DIG}=$ digitate, $\mathrm{BAR}=$ barrel, $\mathrm{CUS}=$ cushion, $\mathrm{GLO}=$ globular. 


\subsubsection{Contribution of growth forms to complexity metrics}

Analysis of the coefficients of LASSO linear regression showed that in coral-dominated quadrats cover of massive corals had a positive effect on rugosity (i.e. a negative effect on $R$ ), whereas cover of encrusting, sub-massive and tabular corals had a negative effect (Fig. 5.9-A). Cover of branching coral had a positive effect on $1 / k$, whereas tabular coral cover had a negative effect. No coral growth form was a good predictor of $D_{1}$ and $D_{2}$ (larger refuge holes, 120-60 $\mathrm{cm}$ and 60-30 $\mathrm{cm}$ respectively). Cover of branching, foliose and massive corals had a positive effect on $D_{3}$ (30-15 cm refuge holes), whereas cover of encrusting, sub-massive and tabular corals had a negative one. Cover of branching and massive corals had a positive effect on $D_{4}$ (15-5 cm refuge holes), while cover of encrusting, sub-massive and tabular corals a negative one. Finally, only cover of branching corals had a positive effect on $D_{5}(5-1 \mathrm{~cm}$ refuge holes), whereas cover of sub-massive and tabular corals had a negative one. In spongedominated quadrats, few growth forms could consistently predict complexity metrics (Fig. 5.9B). Barrel sponge cover had a positive effect on rugosity (i.e. a negative effect on $R$ ) and $D_{1}$, but a negative effect on $D_{5}$. Cover of tubular sponges also had a positive effect on rugosity and vector dispersion, but a negative effect on $D_{5}$. Finally, cover of cushion growth forms had a negative effect on $D_{5}$, and cover of branching, digitate and massive sponges a positive one, although weak. For both coral and sponges, the model coefficients that the LASSO algorithm did not shrink to zero had small values, indicating overall weak relationships between specific growth forms and the complexity metrics. No sponge growth form was selected by the LASSO as predictor of the complexity metrics in coral quadrats, and vice versa (results not shown). 

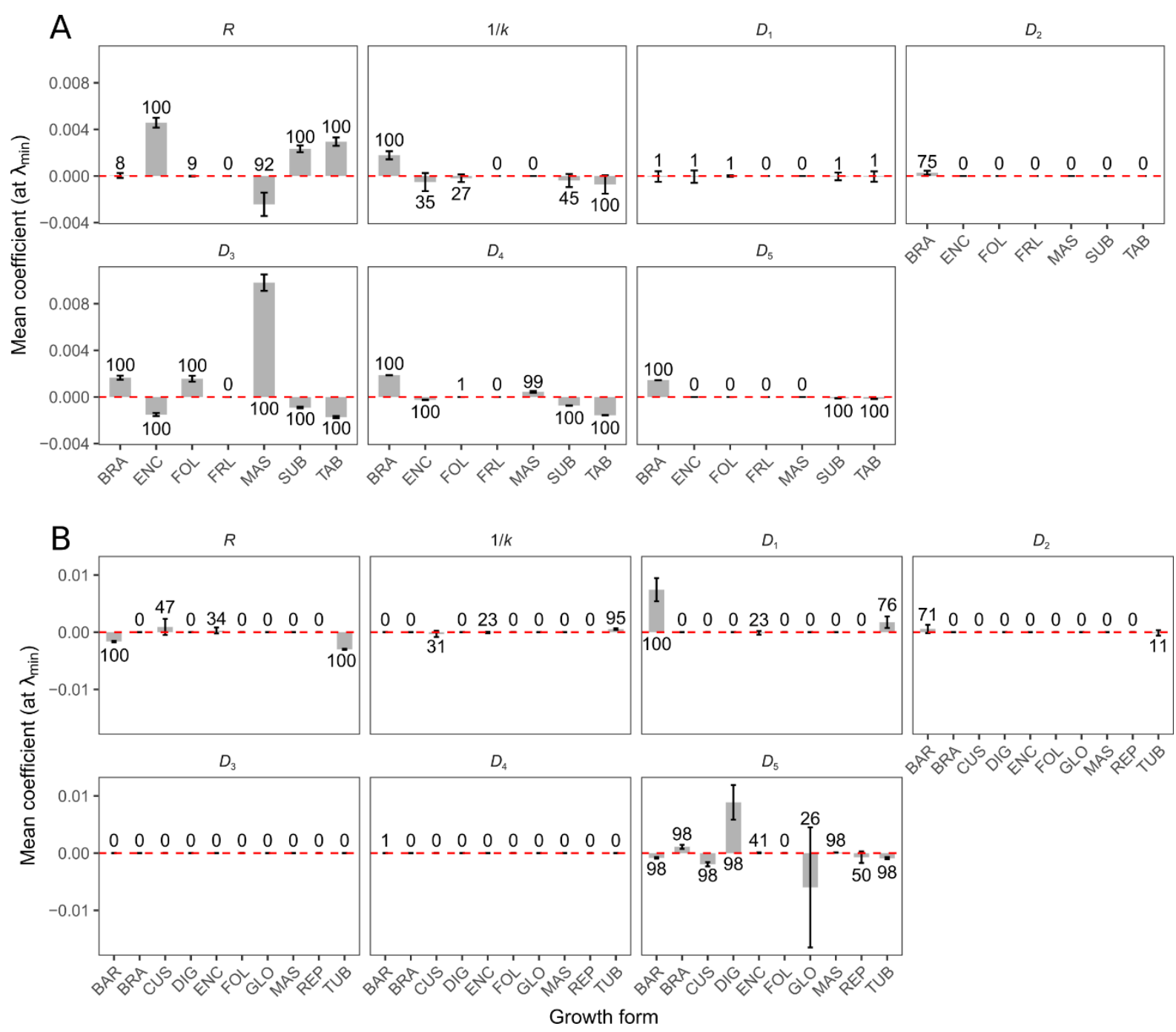

Figure 5.9. Mean values of the LASSO regression coefficients of percentage cover of the growth forms as predictors of the 7 complexity metrics, estimated at $\lambda_{\min }$ in 100 random iterations of the cross-validation. A: coral-dominated quadrats; B: sponge-dominated quadrats. Bars indicate standard deviation, dashed horizontal line represents zero (no effect of the predictor - growth form). The numbers indicate how many random iterations of the crossvalidation returned a non-zero value for the coefficient of that predictor. Only predictors whose coefficients were not shrunk to zero in $95 \%$ of the iterations were considered. $R=$ rugosity (note the inverse scale), $1 / k=$ vector dispersion, $D_{1-5}=$ fractal dimension, $\mathrm{BRA}=$ branching, $\mathrm{TAB}=$ table, $\mathrm{FOL}=$ foliose, $\mathrm{MAS}=$ massive, $\mathrm{SUB}=$ sub-massive, $\mathrm{FRL}=$ free-living, $\mathrm{ENC}=$ encrusting, $\mathrm{TUB}=$ tubular, $\mathrm{REP}=$ repent, $\mathrm{DIG}=$ digitate, $\mathrm{BAR}=$ barrel, $\mathrm{CUS}=$ cushion, $\mathrm{GLO}$ $=$ globular. 


\subsection{Discussion}

In this chapter I compared the structural properties of coral-dominated and sponge-dominated quadrats on an Indonesian coral reef using underwater 3D photogrammetry. The aims of this chapter were to identify differences between the structural complexity of coral-dominated and sponge-dominated reef quadrats, and to understand which sponge growth forms contribute to reef structural complexity. I found that sponge-dominated quadrats where upright sponges were abundant presented a complex 3D framework, comparable to that of coral-dominated quadrats in terms of rugosity, heterogeneity of the surfaces, and largest refuge space complexity (120$60 \mathrm{~cm}$ ). However, sponge-dominated quadrats were more complex at the second largest scale of refuge spaces $(60-30 \mathrm{~cm})$, mostly due to barrel sponges, whereas coral-dominated quadrats were more complex at the smallest scale of refuge spaces $(5-1 \mathrm{~cm})$, mostly due to branching corals. I also found that contributions of single growth forms to specific complexity metrics were generally weak, indicating that reef complexity is provided by the combination of many shapes of benthic organisms, rather than by one particular coral or sponge morphology. These results indicate that a loss of coral and sponge diversity will result in reduced structural complexity, and that a decline in corals and a corresponding increase in sponge dominance will likely correspond to an increase in larger refuge holes $(60-30 \mathrm{~cm})$ and to a loss of smaller refuge holes $(5-1 \mathrm{~cm})$. The latter has potentially negative implications for coral reef fauna that depends on small refugia, like small cryptobenthic reef fish that have a central role in reef food webs.

\subsubsection{Structural properties of coral and sponge quadrats}

At high structural complexity, five complexity metrics out of seven $\left(R, 1 / k, D_{1}, D_{3}\right.$ and $\left.D_{4}\right)$ did not differ between coral-dominated and sponge-dominated quadrats. This showed that, on sponge-dominated reefs, sponges with complex morphologies can provide a 3D framework that is largely comparable to that of corals on coral-dominated reefs. However, coral-dominated quadrats showed higher structural complexity at the smallest scale of refuge spaces $\left(D_{5}\right.$, refuge holes $5-1 \mathrm{~cm}$ in diameter) compared to sponge-dominated quadrats. Conversely, spongedominated quadrats showed higher complexity at the second largest scale of refuge spaces $\left(D_{2}\right.$, refuge holes $60-30 \mathrm{~cm}$ in diameter).

Three-dimensionally complex coral growth forms, like branching and foliose colonies, were the most abundant coral growth forms in high-complexity coral quadrats. With a morphometric approach, Richardson et al. (2017) showed that branching Porites on Lizard Island (Great Barrier Reef) were the most complex habitats at the smallest spatial scale $(4 \mathrm{~cm}$, comparable 
to my smallest scale of refuge spaces of 5-1 cm), thus making a major contribution to smallscale habitat structure on the reef. In the present study, while ramified sponge morphologies (like branching and repent) were present in high-complexity sponge quadrats, either their abundance was too low to offer small-scale habitat, or sponges lacked the inherent morphological complexity of branching coral colonies.

Branching corals are beneficial to reef health and resilience, by promoting abundance and diversity of associated vertebrates (Huntington et al. 2017), and by increasing settlement rates of juvenile fish and attracting fish predators (Shima et al. 2008). Recent research highlighted the crucial role of small-sized cryptobenthic reef fishes in the reef trophodynamics, where they produce $\sim 60 \%$ of the consumed reef-fish biomass (Brandl et al. 2019) and account for almost half of the global reef fish biodiversity (Brandl et al. 2018). Cryptobenthic reef fish families have a prevalence $(>10 \%)$ of species with body length $<50 \mathrm{~mm}$ (Brandl et al. 2018). The body size of these fish species coincides with the refuge spaces of the smallest size considered in the present study $(5-1 \mathrm{~cm})$. Therefore, with the ongoing decline of corals and the associated flattening of coral reefs (Alvarez-Filip et al. 2009), the disappearance of small-scale habitats $(5-1 \mathrm{~cm})$ linked to corals and the inability of sponges to compensate for it will likely have disproportionate negative consequences for coral reef fish assemblages (Emslie et al. 2014). Differences in how reef fauna is associated with the habitat provided by coral-dominated and by sponge-dominated reefs have not been investigated. However, due to the loss of small refuge spaces $(<5 \mathrm{~cm})$, it is likely that even highly complex sponge reefs will have a different associated fauna, with possible consequences for the food web.

While many studies have discussed the role of sponges as source of habitat (e.g. Ryer et al. 2004, Sharf et al. 2006, Fiore and Cox Jutte 2010, Beazley et al. 2013, Kenchington et al. 2013, Gerovasileiou et al. 2016), the contribution of sponges to seafloor structural complexity has been mostly explored for systems where sponges have very high benthic dominance (Maldonado et al. 2016). Dense aggregations of sponges are recognised as sources of habitat heterogeneity in many ecosystems (Buhl-Mortensen et al. 2010, Maldonado et al. 2016). For example, Conway et al. (2001) reported hexactinellid sponge reefs from British Columbia elevating up to $1.5 \mathrm{~m}$ above the underlying abiotic reef matrix. Deep-sea sponge beds supply 3D relief to the seafloor in the North-Atlantic (ICES 2009, Hogg et al. 2010) and Australian deep continental shelf (Fromont et al. 2012), while upright sponge morphologies contribute, with macroalgae, to the structural complexity of temperate estuaries (van Lier et al. 2017). The role of sponges as providers of structural complexity on coral reefs, however, has never been 
investigated. In the present study, the fractal dimension metric $D_{2}$ (refuge holes $60-30 \mathrm{~cm}$ in diameter, the second largest category) was higher in sponge quadrats than in coral quadrats, at high complexity. Barrel and tubular sponge morphologies were the most abundant upright sponge growth forms in high-complexity sponge quadrats. Conspicuous sponge species, like the barrel sponge Xestospongia, can reach large sizes (often $>1 \mathrm{~m}$ in both diameter and height) and therefore contribute to reef structure (McMurray et al. 2008, McGrath et al. 2018). Bell (2007) proposed that, in the Wakatobi, barrel sponges disrupt the hydrodynamic flow with their structure, and that massive chimneys and tubes (collectively defined as tubular in the present study) are among the morphologies providing 3D structure and therefore microhabitat. The spaces between large barrel or tube sponge growth forms and the neighbouring benthic organisms explained the higher values of $D_{2}$ for sponge quadrats compared to coral quadrats, as well as the rugosity and heterogeneity of the reef surface.

However, the relative lack of the second largest refuge holes $\left(D_{2}\right)$ in coral-dominated quadrats compared to sponge-dominated quadrats was probably due in part to the underrepresentation in the models of the overhang spaces provided by table corals. Overhang space under table corals could not be captured with my application of the method of Young et al. (2017) because table corals were parallel to the seafloor, and so was the action camera (as necessary for correct model rendering). Furthermore, the upper surface of table corals is essentially flat, and therefore these structures give limited contribution to both rugosity $(R)$ and heterogeneity of the surfaces $(1 / k)$. This highlights the need to compound popular measures of structural complexity, like rugosity, with metrics that capture fractal properties of the reef (Reichert et al. 2017). Tabular corals are keystone structures on coral reefs, and the overhanging space beneath them is used by large reef fish as shelter, as advantageous point for ambush predation, and as source of shade (Kerry and Bellwood 2012, 2015a, b). The low contribution to reef complexity of tabular corals in the present study was therefore a result of the filming technique and of the metrics used to quantify complexity. For reef areas where table corals are abundant, other methods of measuring structural complexity in situ, such as visual evaluation of structural variables with Habitat Assessment Scores (Gratwicke and Speight 2003), may be used to complement the information provided by the 3D photogrammetry.

Notably, at low levels of structural complexity, four of the seven complexity metrics $(R, 1 / k$, $D_{3}$ and $D_{4}$ ) indicated that sponge-dominated quadrats were more complex than coral-dominated quadrats. Furthermore, for sponge quadrats, the increase in rugosity and vector dispersion from low to high complexity was small (although significant) compared to that of coral quadrats, 
and the increase in $D_{3}$ and $D_{4}$ was not significant. That is, based on more than half the complexity metrics, the difference in overall structural complexity between low- and highcomplexity sponge-dominated quadrats was subtler than for coral-dominated quadrats. Similarity in the complexity metrics between low- and high-complexity sponge-dominated quadrats was due to a somewhat similar morphological composition of sponge assemblages across all sponge-dominated quadrats, with encrusting morphologies always being the most abundant sponge growth form. At low complexity, higher values of $R, 1 / k, D_{3}$ and $D_{4}$ in sponge quadrats than in coral quadrats were due to the presence of cushion and massive sponge growth forms. While these morphologies do not provide any small-scale structural complexity, they are compact, amorphous three-dimensional growth forms with some degree of relief (BouryEsnault and Rützler 1997) and thus contribute to the overall reef framework. Low-complexity coral quadrats, on the other hand, were dominated by encrusting corals (essentially twodimensional), with the sporadic presence of table corals (which due to the issues discussed above are effectively a flat surface if looked at from above, and therefore do not contribute majorly to rugosity and vector dispersion).

\subsubsection{Contribution of morphologies to overall 3D structure and to complexity metrics}

For both coral-dominated and sponge-dominated quadrats, few growth forms explained, alone, a significant amount of variation in overall reef structural complexity (branching and massive for corals, barrels for sponges). However, while the coefficients estimated by the LASSO for coral and sponge morphologies as predictors of complexity metrics helped to discern the effects of single growth forms on reef structural complexity, such effects were generally weak. Weak effects of individual growth forms on structural complexity suggests that, while upright morphologies of benthic organisms are required to maintain the 3D structure of the reef, structural complexity is a property of coral reefs that is provided by the combination of many growth forms (Darling et al. 2017).

In sponge quadrats, only barrel sponges explained significant variation in overall reef structural complexity, demonstrating the key role of these large sponges as providers of reef elevation and structural heterogeneity (Bell 2007, McMurray et al. 2008). Specifically, barrel sponges were good predictors of rugosity and complexity at large scale of refuge spaces (120$60 \mathrm{~cm}$ ), but not of heterogeneity of the surfaces and of complexity at smaller scale of refuge spaces, in fact contributing negatively to the smallest scale of refuge spaces, $D_{5}(5-1 \mathrm{~cm})$. Tubular sponges contributed to rugosity $(R)$ and to heterogeneity of the surfaces $(1 / k)$, but were 
negatively associated with small scale of refuge spaces. Complexity at small scale of refuge spaces in sponge quadrats $\left(D_{5}\right)$ was provided mostly by branching and digitate sponges, but it should be noted that the relationships between these morphologies and $D_{5}$ were weak. Furthermore, only barrel sponges explained variation in overall structural complexity, indicating that the contribution of other sponge morphologies to reef architecture was limited.

The way barrel sponges contributed to structural complexity in sponge quadrats was similar to that of massive corals in coral quadrats. Massive coral colonies in the present study contributed to rugosity and, weakly, to complexity at intermediate- to small scales of refuge spaces (30-5 cm, $D_{3}$ and $\left.D_{4}\right)$, but not to heterogeneity of the surfaces $(1 / k)$, or to complexity at smaller scales of refuge spaces $\left(5-1 \mathrm{~cm}, D_{5}\right)$. Contributions of different coral morphologies to different scales of refuge spaces is in agreement with Richardson et al. (2017), who showed that colonies of branching Porites form complex habitats at small spatial scales $(4 \mathrm{~cm})$, whereas massive Porites are more complex at larger spatial scales of $16-64 \mathrm{~cm}$.

In coral-dominated quadrats, massive and branching coral morphologies contributed the most to the variation in overall structural complexity. Previous research has identified branching corals as primary contributors to reef complexity (e.g. Denis et al. 2017, Huntington et al. 2017). Cover of encrusting, submassive and table corals did not explain significant variability in the 3D structure of the quadrats, and these morphologies were negatively associated to most complexity metrics. While, as discussed, the association of table corals with low structural complexity reflects the method applied rather than the true role of these structures as source of complexity, none of these coral morphologies offered heterogeneous $3 \mathrm{D}$ relief to the substrate.

Importantly, if reefs become dominated by sponges, complex sponge morphologies will be required for structural complexity to be maintained. Drivers of morphological diversity of coral reef sponge assemblages in the Indo-Pacific are not well known. Barnes and Bell (2002) reported water-flow regimes and substratum heterogeneity as key drivers of morphological diversity of Indian Ocean sponge assemblages, and morphological diversity of temperate sponges is influenced by local environmental conditions like sedimentation or currents (Bell and Barnes 2000c). However, no clear relationship was found between sponge morphological diversity and site-specific conditions in the Wakatobi (Bell 2007). Storms can damage tall sponges on coral reefs (Mercado-Molina and Yoshioka 2009), and with the frequency of reefthreatening storms projected to increase with climate change (Cheal et al. 2017), complex sponge morphologies may be limited to low-energy environments on future reefs. Moreover, 
at a degraded reef site in the Wakatobi, the encrusting phototrophic sponge Lamellodysidea herbacea has recently increased in abundance (Biggerstaff et al. 2017b). Dominance of these low-complexity sponge growth forms after coral decline would result into a loss of structural complexity. However, at the same coral-depleted Wakatobi reef where L. herbacea abundance has increased, barrel sponges are a conspicuous and abundant sponge species across depths (McGrath et al. 2017, 2018). Sponge-provided structural complexity on transitioning reefs will depend on which sponge species and growth forms will be dominant.

Although corals or sponges were always dominant benthic groups in the quadrats, other benthic organisms had the potential to contribute to structural complexity. While this may have made the contributions of specific coral and sponge morphologies to complexity metrics harder to measure, it is also a realistic representation of a reef: a complex composition of many benthic organisms growing into different shapes. Confounding effects of other benthic groups on structural complexity were mitigated by placing the quadrats in such way that the dominant organism was always far more abundant than the rest of the benthos with an upright structure. Furthermore, I tested for the contribution of sponges to structural complexity in coral quadrats, and vice versa. The non-significant results of these analyses and the low percentage cover of other upright sessile organisms suggested that non-dominant benthic groups were unlikely to contribute significantly to reef structure.

Importantly, while the maintenance of structural complexity of the reef depends on live corals producing new carbonate (Darling et al. 2017), dead coral skeletons also contribute to reef architecture (Lindahl et al. 2001). The confounding effects of the possible contribution of dead coral skeletons to habitat complexity in the quadrats were negligible, because of the very low mean dead coral cover $(<0.5 \%)$. The underlying reef geology is also an important determinant of overall rugosity and large-scale structural complexity of the reef (Kleypas et al. 2001). Quantifying the contribution to structural complexity of the underlying abiotic matrix versus that of benthic organisms was not possible with my approach. However, all quadrats were placed on the reef slope haphazardly with respect to local reef aspect and geological heterogeneity, following the only criterion that either corals or sponges were the dominant benthic organism. Therefore, it is unlikely that the abiotic matrix contributed to overall complexity in a different way between coral-dominated and sponge-dominated quadrats.

The present study shows that high density of sponges with erected growth forms contribute to overall reef structural complexity in a way that is similar to that of corals. However, sponge- 
dominated reef areas lack the smallest scale of refuge space measured $(5-1 \mathrm{~cm})$ that complex coral growth forms provide. The loss of 5-1 cm refuge spaces has important implications for coral reef trophodynamics on reefs where sponges will become dominant after corals have declined (Chapter 2, Bell et al. 2018a). Low structural complexity at small spatial scales on sponge reefs would have a negative impact on small reef fish that are central to coral reef food webs (Brandl et al. 2019). Furthermore, coral reef structural complexity relies on live corals producing new carbonate reef matrix (Darling et al. 2017). While complex sponge morphologies would offer 3D structure to the reef as long as the sponges live, dead coral reef sponges do not leave behind a limestone skeleton like corals do, and do not contribute to reef accretion. In fact, future increase in the abundance of bioeroding sponges may actively contribute to reef erosion (Schönberg et al. 2017), with an overall negative effect on habitat complexity. Therefore, it is likely that sponges will not provide long-term maintenance of structural complexity of coral reefs. Further research is needed to understand the specific consequences that changes in structural complexity associated with an increase in sponge dominance will have on coral reef fauna. Future studies should focus on the relationships between reef fishes and invertebrates and different sponge morphologies.

The results from this Chapter guided the modelling of sponges in the Atlantis model (Chapter 6) by providing insight on the role of sponges as source of structural complexity on coral reefs. Atlantis is already equipped to capture the rugosity associated with corals, and the effect of this rugosity on other ecological processes (for example fish recruitment, see Audzijonyte et al. 2019). New code was developed to allow for sponge-provided structural complexity (see Chapter 6), which can be parameterised as appropriate depending on the sponge morphological diversity that each future Atlantis application will capture. The role of sponges as suppliers of structural complexity was highlighted as important by qualitative modelling results in Chapter 2. 
Chapter 6. A framework to model sponges in the ecosystem model Atlantis 


\begin{abstract}
Ecosystem models capture the physical and biological components of entire ecosystems, with the option to include the human use of natural resources. Ecosystem models can be used to simulate future ecological scenarios, and to predict trajectories of change when ecosystems are exposed to perturbations. As such, ecosystem models of coral reefs can help us gain insight on the effects of global climate change and local environmental pressure on these complex ecosystems. Sponges are an important component of coral reefs, but to date there is limited information on many aspects of sponge ecology, and sponges rarely have high commercial value on coral reefs. For these reasons, detailed representations of sponges in ecosystem models are missing. In this Chapter, I present the development of a framework to include sponges as three functional groups (heterotrophic, phototrophic, and bioeroding) in the ecosystem model Atlantis. Based on previous data chapters in this thesis, I developed new Atlantis code for sponge-specific ecological processes. These processes include benthic competition with corals and macroalgae, sponge-mediated bioerosion of carbonates and contribution to structural complexity, and Silicon-limitation to sponge growth. In addition, existing Atlantis code was adapted to allow for the response of sponges to ocean warming and acidification. This new framework to model sponges was tested in an Atlantis application for the Australian Great Barrier Reef (GBR). Sponges were parameterised in Atlantis GBR with percent cover data from the Australian Institute of Marine Science, and by searching the literature for information on life history traits and response to climate change of GBR sponges. Model development and calibration are presented. At the time of completing this thesis, Atlantis GBR is undergoing model calibration, and results from model runs will be presented elsewhere.
\end{abstract}

\title{
6.1. Introduction
}

Ecosystem models that capture entire ecosystems, including biological, oceanographic, and socio-economic components, are also referred to as end-to-end models. Atlantis is a spatiallystructured, deterministic end-to-end model used for the dynamic modelling of entire ecosystems, written in the programming language $C$ (Fulton et al. 2011). The model tracks biomass (nitrogen) flows in a 3D model domain. The modelled area is divided horizontally into boxes, and each box is divided into a number of vertical layers that depend on depth. Hydrodynamic flows between boxes in Atlantis are often derived from finer-scale oceanographic models, with nutrients and plankton being passively transported with water, 
while other living groups can move independently from hydrodynamic flows. Atlantis is a sophisticated and versatile ecosystem model that can provide useful insights into how an ecosystem can respond to environmental change and anthropogenic pressure (such as fisheries) given a large number of complex dynamic interactions in the system (Audzijonyte et al. 2019). Because of its potential to represent entire ecosystems and simulate management strategies and their outcomes, Atlantis is most useful in the strategic evaluation of ecosystem-based management strategies, with a strong focus on fisheries (Link et al. 2010, Fulton et al. 2011). Atlantis has been used to evaluate the effects of environmental change on ecosystems all over the world, including the Northeast United States (Nye et al. 2013, Fay et al. 2017), the Chesapeake Bay (Ihde and Townsend 2017), the Pacific coast of the United States (Marshall et al. 2017), South Africa (Ortega-Cisneros et al. 2018), and Southeast Australia (Griffith et al. 2011, Fulton et al. 2018).

End-to-end models like Atlantis (and ecological models in general) are an approximation of the real world. There is a practical limit to model realism that is dictated by the complexity of the interactions between model components, and by the state of the knowledge of such components and interactions (see Collie et al. 2014). For this reason, ecological models often represent some components with higher realism than others, depending on the research questions being considered. A species of high economic value or ecological importance in a certain ecosystem may be explicitly represented and carefully parameterised, whereas many species of no commercial interest or that are not the focus of the investigation may be aggregated into functional groups (for example, see detailed prawn groups and aggregated benthic groups in Gribble 2003).

Partly due to a paucity of data and information, and partly due to their often-overlooked ecological importance, sponges in ecosystem models have often been represented as generic benthic filter feeders, indistinguishably from other benthic organisms. For example, in the Atlantis model for Guam, sponges are aggregated into one group of filter feeders together with octocorals, tunicates, zoanthids, giant clams, bivalves, polychaetes, foraminifera, bryozoans, and brittle stars (Weijerman et al. 2015). Similarly, ecosystem models for the North-West Australian continental shelf have aggregated sponges and coral as generic "megabenthos" (InVitro, Fulton et al. 2006) or sessile epibenthos (Bulman et al. 2006), despite sponges being abundant and providing important habitat on the Australian shelf (Fromont et al. 2012). 
Sponges have been modelled as single model group in some ecosystem models, in particular in tropical or sub-tropical applications where sponges are abundant like the Gulf of Mexico (Atlantis, Ainsworth et al. 2015), and the Caribbean (Ecopath with Ecosim, Alva-Basurto and Arias-González 2014). Some model applications have focused on some of the ecological roles of sponges, including competition with corals and macroalgae (González-Rivero et al. 2011), the sponge loop (Silveira et al. 2015), and sponge-mediated carbon trophic pathways (McMurray et al. 2017). However, many functional roles of sponges vary between sponge species. For example, not all sponges are bioeroders, and sponge-mediated carbon pathways in the food web depend on the trophic mode of the sponges in the ecosystem (Wilkinson 1987). Furthermore, experimental evidence has shown that heterotrophic and phototrophic sponges will likely respond differently to climate change, with OA potentially alleviating the effects of warming for phototrophic species but exacerbating them for heterotrophic species (Bennett et al. 2017). Similarly, bioeroding sponges may be advantaged under OA conditions (Fang et al. 2013, Wisshak et al. 2013, Stubler et al. 2014), although increased temperature may cause metabolic stress, bleaching and overall decreased fitness (Fang et al. 2014, Ramsby et al. 2018).

Sponges have many important ecological roles on coral reefs and in other ecosystems (see Chapter 1, Wulff 2006a, and Bell 2008). Many sponges will likely be more tolerant than corals to projected conditions of warming and acidification (Bell et al. 2013, 2018b), and sponges with different functional roles and trophic strategies will likely be affected in different ways by future environmental change (see Chapter 1). Because of the many important ecological roles of sponges, future changes in sponge dominance on coral reefs may have important flow-on effects on the ecosystem (see Chapters 1 and 2). For these reasons, ecosystem model applications that (1) focus on coral reefs or ecosystem where sponges are present and potentially abundant, and (2) are used to explore the response of ecosystems to environmental change, should capture 3 sponge groups: heterotrophic sponges, phototrophic sponges, and bioeroding sponges. Modelling sponges as multiple functional groups can provide useful insights into the development of benthic communities under future scenarios.

In this Chapter, a novel framework for the modelling of sponges in Atlantis is presented. Previous data chapters in this thesis guided the development of the sponge components of Atlantis. The results of the qualitative modelling in Chapter 2 indicated the key aspects of sponge ecology that may play an important role in regime shifts towards sponge-dominated reefs. These processes, which included benthic competition with corals and macroalgae, the role of the sponge loop on future reefs, and trophic relationships between sponges and sponge 
predators, were captured in the novel framework to model sponges in Atlantis presented here. Results of the temporal analysis of the Indonesian sponge assemblage (Chapters 3 and 4) highlighted high temporal variability of sponge abundance, and of percentage cover of sponges, algae and other benthic invertebrates on coral reefs. These temporal analyses aided me in discerning to what extent sponges and other benthic groups may fluctuate in Atlantis simulations. Therefore, the results from Chapters 3 and 4 were useful to qualitatively validate temporal patterns observed in Atlantis runs, during the process of model calibration. In addition, mixed models in Chapter 4 suggested that turf algae, whilst being efficient colonisers on the reef within one year from the opening of free substrate, may be outcompeted by other benthic groups, including sponges. Therefore, the Atlantis code was modified to allow for parameterizable effects of the cover of turf algae and CCA on the settlement and growth of corals and sponges (see Chapter 4 and references therein). Finally, 3D photogrammetry from Chapter 5 showed that some coral reef sponges may provide reef rugosity similar to that of corals, although sponges are unlikely to provide small refuge holes $(5-1 \mathrm{~cm}$ in diameter) like branching corals do. This contribution of sponges to coral reef structural complexity was therefore included into Atlantis as new code, providing a flexible and fully parameterizable framework to model different degrees of contribution of sponges to reef structural complexity.

In addition, my work also contributed to the development of an Atlantis model for the Great Barrier Reef (GBR), which has been developed by the Commonwealth Scientific and Industrial Research Organisation (CSIRO, Australia). Specifically, my work contributed to the development of Atlantis GBR in four ways: (1) by providing a novel framework to model sponges; (2) by informing the model with benthic cover data from the Long-term Monitoring Program (LMTP) conducted by the Australian Institute of Marine Science (AIMS); (3) by contributing to the parametrisation of the benthic groups of the model; and (4) by participating to model calibration.

This Chapter is structured differently from the previous 4 data chapters, as it illustrates the design of the sponge components of Atlantis and the parametrisation of the benthic components of Atlantis GBR. Results and discussion of model simulations are not included in this Chapter, because Atlantis GBR was still undergoing calibration at the time this thesis was completed. 


\subsection{Sponges and sponge processes in Atlantis}

\subsubsection{Sponges in Atlantis}

So far, Atlantis has allowed the modelling of sponges as a biomass pool of benthic consumers, largely feeding on detritus, bacteria and plankton groups. Briefly (see the Atlantis User Guide in Audzijonyte et al. 2019 for details), the biomass pool of a consumer $C x$ in Atlantis is assumed to have no size or age structure, and its biomass changes in time according to its growth from heterotrophic feeding $\left(\mu_{C x}\right)$, its natural mortality $\left(M_{C x}\right)$, the proportion of it that gets eaten by each predator $\left(P_{C x, i}\right)$, fishing mortality $\left(F_{C x}\right)$, and optional encystment terms $\left(\mathrm{Ec}_{\text {out }}\right.$ and $\left.\mathrm{Ec}_{\mathrm{in}}\right)$ :

$$
\frac{\mathrm{d}(C x)}{d t}=\mu_{C x}-M_{C x}-\sum_{i=\text { predator groups }} P_{C x, i}-F_{C x}-E_{C x_{\text {out }}}+E_{C x_{\text {in }}}
$$

Corals in Atlantis are modelled as consumers, but they are an age-structured biomass pool, which allows the separate modelling of young recruits and larger colonies. Briefly, Atlantis captures the most important ecological and physiological functions of corals, including: photosynthesis of associated symbiotic microalgae; calcification; contribution to reef rugosity and the use of this as refuge space by fishes; bleaching as response to thermal stress; both local and regional recruitment as a result of brooding and broadcasting reproductive strategies respectively; and growth into size classes (see Weijerman et al. 2014, 2015 for detailed description of how corals are modelled in Atlantis).

Because sponges have some similarities with corals (for example, they are both benthic invertebrates with mixed trophic modes and reproductive strategies), the sponge framework was based on the coral framework already present in the Atlantis code, with modifications and additions that would allow the modelling of sponge-specific processes. Sponges were modelled as age-structured biomass pool of benthic consumers with two life stages (loosely defined "juvenile" and "adult"), similar to corals. Sponge groups in Atlantis can rely on filter-feeding and photosynthesis in user-defined proportions, thus allowing the modelling of heterotrophic and phototrophic (or mixotrophic if relying on both feeding modes, as all sponges are capable of heterotrophic feeding) sponges. Sponges can bleach when exposed to thermal stress (only phototrophic and mixotrophic), they compete with other benthic organisms, they contribute to reef rugosity but also destroy it (only bioeroders), and may be silicon-limited (but see Pawlik and McMurray 2020 for discussion on silicon limitation of sponges). An overview of the modelling of sponge ecological processes in Atlantis is reported here, and readers should see 
Weijerman et al. (2015) and the Atlantis User Guide (Audzijonyte et al. 2019) for details on how to model coral and sponge groups in Atlantis.

Sponge biomass in Atlantis changes over time according to Eq. 1, except sponge growth depends on ingested biomass and on the contribution from photosymbionts (similarly to corals, see Weijerman et al. 2015 and Audzijonyte et al. 2019 for details). The user can set the proportion of the respective contributions of these two feeding modes for each sponge model group, depending on whether the group represents heterotrophic, phototrophic, or mixotrophic (i.e. a blend of both) sponges. Information on temporal variability of sponge abundance and percentage cover reported in Chapters 1 and 2 was used for qualitative validation of the modelled dynamics of sponge biomass and proportional cover produced by Atlantis.

Many of the functions already used to model corals in Atlantis could be used, unmodified, to model sponges too, simply requiring a sponge-specific set of parameters. However, some of the coral code had to be modified in order to adequately represent both corals and sponges. For example, coral growth and calcification rates are limited in Atlantis by the amount of energy that corals use for calcification, with coral growth stopping for aragonite saturation $<1.0$ (Weijerman et al. 2015). Because most sponges use silicon to build spicules, sponge growth was not limited by the conditions of water aragonite saturation, but silicon limitation to sponge growth was introduced (see below). Similarly, the code for the effects of sedimentation on corals were modified to allow a flexible representation of sediment-mediated smothering of these two groups.

\subsubsection{Benthic competition: growth limitation and smothering}

Growth of benthic organisms (e.g. coral, algae, seagrass, and benthic filter-feeders) in Atlantis is limited by the amount of available substrate that can be used for horizontal expansion (see Audzijonyte et al. 2019 for details). Furthermore, simulating intra-specific density dependence, a crowding effect is also applied to the growth of benthic organisms, based on a predetermined value of maximum biomass per unit space (carrying capacity). These are limitations to the growth of benthic organisms that depend on the available substrate, rather than explicit competition for space. In addition, Atlantis captures specific interactions between hard corals and phytobenthos. Namely, coral growth in each model box is limited in the model by macroalgae and aided by turf and crustose coralline algae (CCA). This represent the preemption of available substrate from macroalgae (see Hughes 1994), and facilitation to settlement and growth offered to corals by CCA (e.g. Heyward and Negri 1999). Appropriate 
parametrisation determines the degree of competitive prevalence of macroalgae, and the aiding effect of turf and CCA. In addition to pre-emptive competition, macroalgal-mediated smothering of corals is also modelled in Atlantis.

Qualitative model results from Chapter 2 showed that competitive interactions between benthic organisms influence the response of coral reef communities to perturbation. Therefore, it was desirable to make benthic competition more flexible in a way that it could explicitly model competitive interactions between sponges and macroalgae, and between sponges and corals. The existing Atlantis code for benthic competition was expanded to allow for: (1) competitive prevalence of macroalgae over sponges (González-Rivero et al. 2011, 2012, 2016); (2) aiding effect of CCA on sponge growth and settlement (Whalan et al. 2012); (3) macroalgalmediated smothering of sponges (López-Victoria et al. 2006); and (4) reciprocal growth limitation of corals and sponges. This design reproduces three-way competition between corals, macroalgae, and sponges, with general competitive superiority of macroalgae (GonzálezRivero et al. 2011, Chapter 2, Bell et al. 2018a), and a positive effect on coral and sponge growth from turf algae and CCA (see Chapter 4 and references therein).

\subsubsection{Habitat complexity and bioerosion}

In Atlantis, corals provide bottom rugosity, which is used as measure of structural complexity in the model. In turn, reef rugosity influences predator-prey relationships, by providing refuge space for fish, and by this alleviating predation pressure (Audzijonyte et al 2019). Several alternative models for the contribution of corals to reef rugosity are available in Atlantis. These range from pre-determined contributions of benthic organisms to rugosity to dynamic submodels that capture reef building and bioerosion as function of coral biomass and environmental conditions (Weijerman et al. 2014, 2015).

On coral reefs, corals are considered to be the main ecosystem engineers (Wild et al. 2011). However, upright sponge morphologies on reefs, where sponges are abundant, can provide reef rugosity similar to that of corals (Chapter 5). Furthermore, sponges are an important source of habitat in the deep sea, where stony corals are not present (Fromont et al. 2012). On the other hand, bioeroding sponges excavate carbonates and reduce reef structural complexity (see Rützler 2004), and sponges are thought to have an overall negative impact on coral reef 3D structure (Pawlik and McMurray 2020). I modified the Atlantis code to capture the positive contribution of sponges to structural complexity, as well as the sponge-mediate excavation of coral skeletons and the resulting decline in complexity. 
In Atlantis, one of the existing options to model the change in rugosity $R$ due to the contribution of each coral group $C$ at time step $t$ is after Blackwood et al. (2011):

$$
\frac{d R}{d t}=h_{G} C_{\text {cover }}\left(R_{\max }-R\right)-h_{E}\left(1-C_{\text {cover }}\right)(R-1) \quad \text { Eq. } 2
$$

Where $h_{G}$ is a species-specific rugosity growth rate of $C, C_{\text {cover }}$ is the proportional cover of the live, unbleached coral group $C, R_{\max }$ indicates the highest value of rugosity ( 3 in Blackwood et al. 2011), and $h_{E}$ is a background bioerosion rate implicitly representing excavation from all bioeroding organisms (reviewed in Schönberg et al. 2017). In Eq. 2, only live and unbleached corals are assumed to contribute to an increase in reef rugosity, and bioerosion is assumed to act only on substrates not covered by live and unbleached corals (Weijerman et al. 2015). Bioerosion from scraping herbivores like parrotfish (Bruggeman et al. 1996) is also modelled in Atlantis, as further bioerosion term depending on the abundance of scraping herbivores and their grazing activity.

I expanded Eq. 2 so that it would apply to all model groups $G$ representing corals and sponges, and so that it would allow for sponge-mediated bioerosion of carbonates:

$$
\frac{d R}{d t}=h_{G} G_{\text {cover }}\left(R_{\max }-R\right)-\left[h_{E}\left(1-G_{\text {cover }}\right)(R-1)+h_{S E} S E_{\text {cover }}\left(1-G_{\text {cover }}\right)(R-1)\right]
$$

Where the sponge bioerosion rate $h_{S E}$ represents a bioerosion rate specific to bioeroding sponges, and $S E_{\text {cover }}$ is the proportional cover of bioeroding sponges. This new formulation allows carbonate bioerosion mediated by sponges to be separated from that mediated by other bioeroders (including microborers, bivalves, and other invertebrates, see Schönberg et al. 2017). This distinction is important because of the potential for sponge bioerosion to increase on future reefs as a result of increased sponge abundance (but see Ramsby et al. 2017 for evidence that abundance of bioeroding sponges has not increased ubiquitously over recent decades).

Although abundant upright sponge growth forms can provide overall reef structural complexity similar to that of complex coral growth forms, sponges cannot offer the small-scale structural complexity (i.e. small refugia) that are characteristic of branching coral colonies (Chapter 5). Therefore, Atlantis models that include sponges should be parameterised in a way that accounts for the overall lower contribution to reef rugosity of sponges compared to corals (see Audzijonyte et al. 2019 for rugosity-related Atlantis parameters). 


\subsubsection{Silicon limitation}

Atlantis allows to nutrient limitation to primary production to be modelled (most often nitrogen and silicon limitation, but also phosphorous and carbon limitation in some models, Audzijonyte et al. 2019). Silicon limitation in Atlantis can affect diatom biomass pools by reducing their productivity at low levels of dissolved silicon (DSi). It has been suggested that sponges are under chronic silicon-limitation in modern oceans (Lopez-Acosta et al. 2018), but there is currently no evidence for patterns of silicon-limitation to the distribution of sponge assemblages on coral reefs (Pawlik and McMurray 2020). To allow for limitations to sponge growth in conditions of extreme silicon depletion in seawater, we expanded the formulation for silicon limitation to allow the modelling of the effects of DSi depletion on consumers. There is very limited information on the direct relationships between DSi concentrations sponge growth rates. Therefore, silicon limitation to sponge growth was modelled following the Michaelis-Menten equation for silicon uptake from sponges used by Maldonado et al. (2011):

$$
\delta_{S i}=\frac{V_{\max }[D S i]}{K m+[D S i]}
$$

Where [DSi] is the concentration of dissolved silicon, $V_{\max }$ is the maximum uptake velocity of DSi from sponges (i.e. at saturation of DSi), and $\mathrm{Km}$ is the Michaelis constant (i.e. the concentration of silicon at which uptake velocity is $1 / 2 V_{\max }$ ). The term $\delta_{\mathrm{Si}}$ is then used to multiply net sponge growth rate, similarly to other multipliers used in Atlantis to limit growth (Audzijonyte et al. 2019).

\subsection{Other processes: a sub-model for seagrass cover-to-biomass conversions}

Atlantis includes 4 types of physical habitat: reef (hard bottom), flat (sand), soft (mud), and canyon (hard bottom enhancing productivity). Each of these 4 habitat types is assigned a 0-1 proportion within each Atlantis box. These proportions add up to 1 within a box, and they generally do not change over the simulation (except in case of habitat degradation, Audzijonyte et al. 2019). In addition, epibenthic model groups (e.g. corals, sponges, macroalgae, seagrass, etc.) can be considered biogenic habitat in Atlantis. These biogenic habitats are also assigned a 0-1 proportion in each model box, indicating the fraction of the box area that they occupy (independent from physical habitat, and not restricted to 1 to allow for overgrowth). Modelled biotic groups can have different degrees of association to a certain habitat (both physical and biogenic). Preferred habitats enhance a group's productivity, whereas avoided habitats curb it 
(Audzijonyte et al. 2019). Cover of biogenic habitats in Atlantis is used to calculate species habitat, and is therefore important to model dynamics and spatial distribution of model groups.

In Atlantis, proportional cover of a benthic organism is dynamically calculated by the model as function of the group's biomass. However, determining relationships between biomass and benthic coverage of sessile organisms is often difficult, especially for organisms that grow into complex morphologies like corals, sponges, some macroalgae, and seagrass. Therefore, where studies have formulated and validated a relationship between biomass and benthic cover of an organisms, it is desirable to include this information into Atlantis. We have implemented a simple model to convert seagrass leaf biomass into areal coverage, based on Baird et al. (2016). In each Atlantis box, seagrass proportional cover was modelled as:

$$
S G_{A}=1-\exp \left(-\Omega_{S G} S G_{B}\right)
$$

Where $S G_{A}$ is the proportional cover of seagrass, $\Omega_{S G}$ is a nitrogen-specific leaf area coefficient $\left(\mathrm{m}^{2} \mathrm{~g} \mathrm{~N}^{-1}\right)$, and $S G_{B}$ is the above-ground leaf biomass $\left(\mathrm{g} \mathrm{N} \mathrm{m}^{-2}\right)$. This formulation allows for a more precise in-model calculation of the area occupied by seagrass, which constitute important habitat for marine species and contribute to fishery productivity (see Nordlund et al. 2018), and is therefore important for model dynamics. 


\subsection{Model application: Atlantis Great Barrier Reef}

An Atlantis model for the Great Barrier Reef (Atlantis GBR) has been under development at CSIRO, Australia (Fig. 6.1). In brief, Atlantis GBR is a large Atlantis model consisting of 108 boxes (9 of which are non-dynamic boundary boxes, Audzijonyte et al. 2019), and featuring 87 functional groups ( 3 of which are detrital groups). At present, the model is in the advanced stages of its development, and is currently undergoing calibration.

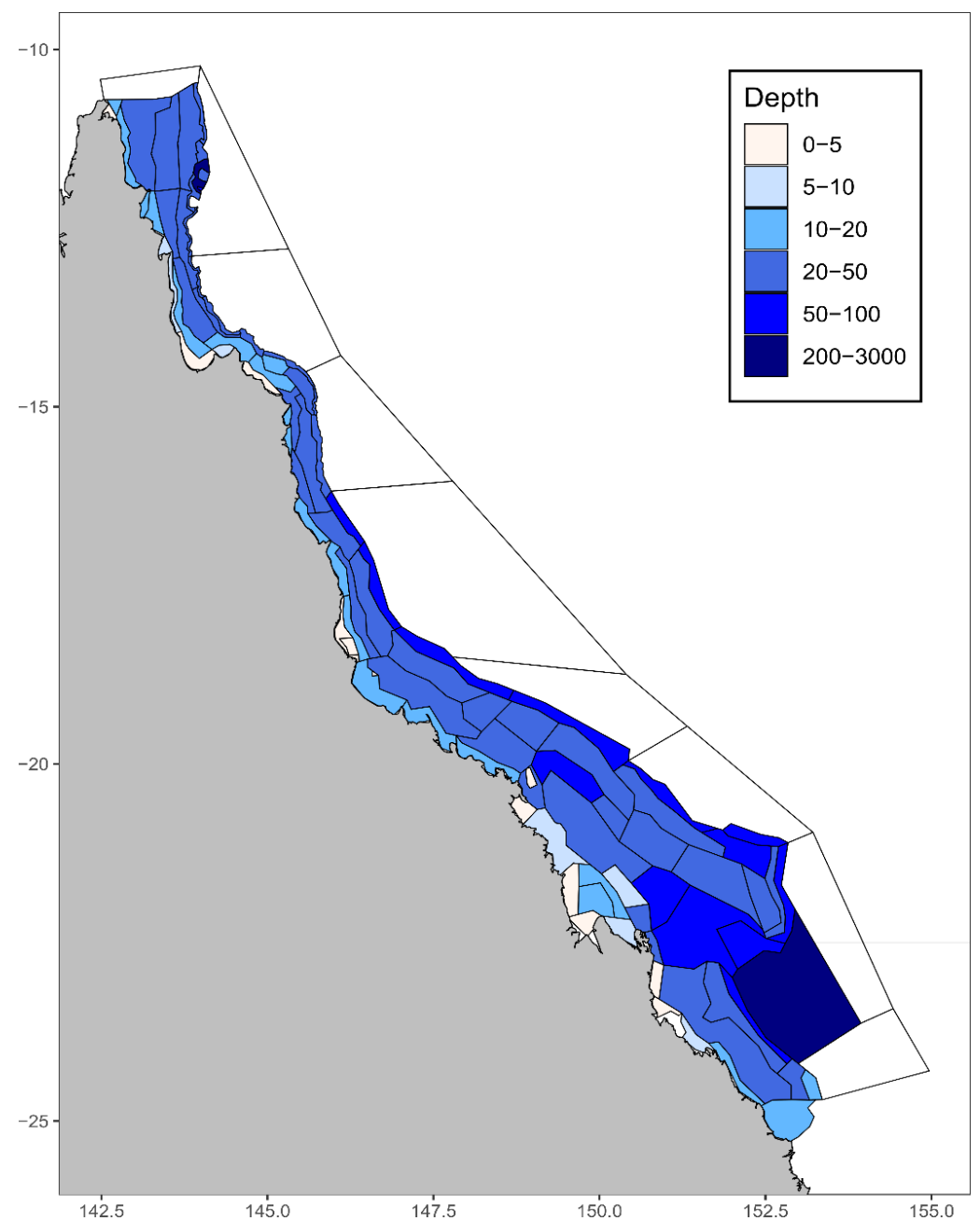

Figure 6.1. Model domain of Atlantis Great Barrier Reef. Colours indicate depth of model boxes. White boxes indicate non-dynamic boundary boxes.

\subsubsection{Cover and biomass of benthic organisms in Atlantis GBR}

Atlantis requires a set of initial conditions to be used as the starting point for model simulations (Audzijonyte et al. 2019). These initial conditions typically describe the ecosystem at a stable state at a certain point in the recent past. Initial conditions include the baseline values for nutrients, biomass pools, benthic cover, other chemical tracers, the number of individuals for the vertebrate groups, etc. Initial conditions are usually obtained from other models (e.g. 
oceanographic models for physical and chemical tracers, biogeochemical models for plankton, etc.), from available datasets, and from the literature. Initial values of proportional cover and biomass of benthic organisms for Atlantis GBR were obtained, with a focus on coral reef areas.

In earlier stages of development of Atlantis GBR, initial values of proportional cover of the benthic organisms in Atlantis GBR had been obtained from one of the datasets collated in the scope of the Great Barrier Reef Seabed Biodiversity Project (SBP, Pitcher et al. 2007). This dataset contained percentage cover data obtained by video tows on inter-reef seabed areas across the GBR. Approx. 1450 sites were sampled across the width and the length of the GBR between 2003-2006, down to a depth of $80 \mathrm{~m}$, and on average $\sim 10 \mathrm{~km}$ apart (Pitcher et al. 2007). However, because the SBP aimed to characterise the previously understudied inter-reef habitats, this dataset contained information on deeper reefs and inter-reef areas, but not on shallow reefs (Pitcher et al. 2007). Coral reefs on the GBR are threatened by climate change and anthropogenic pressure (e.g. Hughes et al. 2017a, 2019), and it is important to understand how this reef system will be altered in the near future (Hughes 2017b). Therefore, benthic cover data from the LTMP conducted by AIMS on the GBR was incorporated into the initial conditions of Atlantis GBR, alongside the SBP data.

\subsubsection{AIMS LTMP cover data: interpolation with Atlantis GBR}

The benthic data from the LTMP conducted by AIMS consists of percentage cover data from 93 GBR reefs sampled across latitudes (N-S), along the continental shelf (inshore-offshore), and over time (data was available for 1994-2016). 3 sites were monitored for each reef, and percentage cover of benthic organisms (Tab. 6.1) was recorded at 5 transects per site. Mean values of percentage cover were calculated at site level, considering transects as replicates. Therefore, this dataset was considerably smaller than the SBP dataset (Fig. 6.2).

AIMS data reported percentage cover of the groups of benthic organisms on GBR reefs, including hard corals (with morphologies), soft corals (with morphologies), sponges, coralline algae, macroalgae, turf algae, and other invertebrates. These groups were mapped to the (coarser) epibenthic groups in Atlantis GBR (Tab. 6.1), and percentage cover data (0-100) were transformed to proportional cover (0-1) to be used in Atlantis. LTMP data from 2005-2006 were used, as nearest to the SBP data collected in 2003-2006. 
Table 6.1. Mapping of the benthic group in the AIMS Long-term Monitoring Program (LTMP) as Atlantis GBR groups.

\begin{tabular}{|c|c|}
\hline AIMS LTMP & Atlantis GBR \\
\hline Reef & Reef \\
\hline Sand & Flat \\
\hline Bottlebrush Acropora & Branching coral \\
\hline Branching Acropora & Branching coral \\
\hline Branching non-Acropora & Branching coral \\
\hline Digitate Acropora & Branching coral \\
\hline Encrusting Acropora & Encrusting coral \\
\hline Encrusting non-Acropora & Encrusting coral \\
\hline Foliose non-Acropora & Encrusting coral \\
\hline Massive non-Acropora & Massive coral \\
\hline Mushroom coral & Massive coral \\
\hline Solitary coral & Massive coral \\
\hline Submassive Acropora & Massive coral \\
\hline Submassive non-Acropora & Massive coral \\
\hline Tabulate Acropora & Branching coral \\
\hline Sponge & Sponge \\
\hline Arborescent and Encrusting Soft Coral & Soft coral \\
\hline Arborescent Soft Coral & Soft coral \\
\hline Capitate Soft Coral & Soft coral \\
\hline Encrusting Soft Coral & Soft coral \\
\hline Lobate Soft Coral & Soft coral \\
\hline Massive Soft Coral & Soft coral \\
\hline Millepora & Soft coral \\
\hline Soft coral & Soft coral \\
\hline Zoanthid & Soft coral \\
\hline Coralline algae & Crustose coralline algae (CCA) \\
\hline Macroalgae & Macroalgae \\
\hline
\end{tabular}




\begin{tabular}{|l|l|}
\hline AIMS LTMP & Atlantis GBR \\
\hline Turf algae & Turf algae \\
\hline Other organisms & $\begin{array}{c}\text { Not mapped to any group as impossible to } \\
\text { determine where to attribute them }\end{array}$ \\
\cline { 1 - 2 } Unknown & \\
\hline
\end{tabular}

The AIMS and SBP datasets were interpolated with the spatial domain of Atlantis GBR (Fig. 6.2), in order to determine: which data points had been sampled on a reef (always the case for AIMS data, but not for SBP data); and which Atlantis GBR boxes contained which data points. This information was used to calculate box-wise mean values of percentage cover of benthic organisms, to be used as initial conditions for simulations with Atlantis GBR. Specifically, SBP data was used to obtain initial values of benthic cover in non-reef areas, and the AIMS LTMP dataset was used for coral reef areas. The analysis presented here was based on the methods previously developed by Shane A. Richards (CSIRO), which were expanded to allow the incorporation of the AIMS LTMP data.

Inter-reef areas. Because of the large size of the SBP dataset ( 1450 sites) and its spatial homogeneity, SBP data was used to calculate mean values of percentage cover of benthic organisms in inter-reef areas for most boxes in Atlantis GBR. For the 11 boxes that did not overlap with either dataset, box-wise mean values of inter-reef benthic cover of neighbouring boxes that had similar characteristics (e.g. depth and latitude) were used. Of these 11 boxes, 9 were in the northern third of the GBR, showing that this region had been less intensively sampled than the rest of the model domain (Fig. 6.2).

Coral reefs. While SBP data covered most of the GBR fairly homogeneously, AIMS data points were sparser and restricted to coral reef habitats, and did not overlap with many Atlantis boxes (Fig. 6.2). As a result, calculating box-wise mean percentage cover of benthic organisms on coral reefs was not possible. Thus, the AIMS dataset (plus the few SBP data points sampled on coral reefs) was used to calculate regional-scale mean values of percentage cover of the main benthic groups on coral reefs. The number of data points, small if compared to the size of the model domain, only allowed to calculate regional mean estimates of percentage cover on reefs for a Northern $(11.77544 \mathrm{~S}-15.81392 \mathrm{~S})$, a central $(15.81392 \mathrm{~S}-19.85240 \mathrm{~S})$, and a Southern (19.85240S - 23.89088S) region of the GBR. According to the same geographic coordinates, the model domain was also divided into 3 sets of boxes. For boxes in the Northern 
third, regional estimates of benthic cover of the Northern reefs were used, and so on for the central and Southern thirds.

Combining the two datasets: The proportion of each Atlantis box occupied by coral reef had been calculated in previous stages of model development. Therefore, the final values of benthic cover to be used as initial conditions in Atlantis GBR were obtained by weighted contributions of the SBP and LTMP datasets, proportionally to the box area covered by nonreef habitat and by coral reefs, respectively (Fig. 6.3).

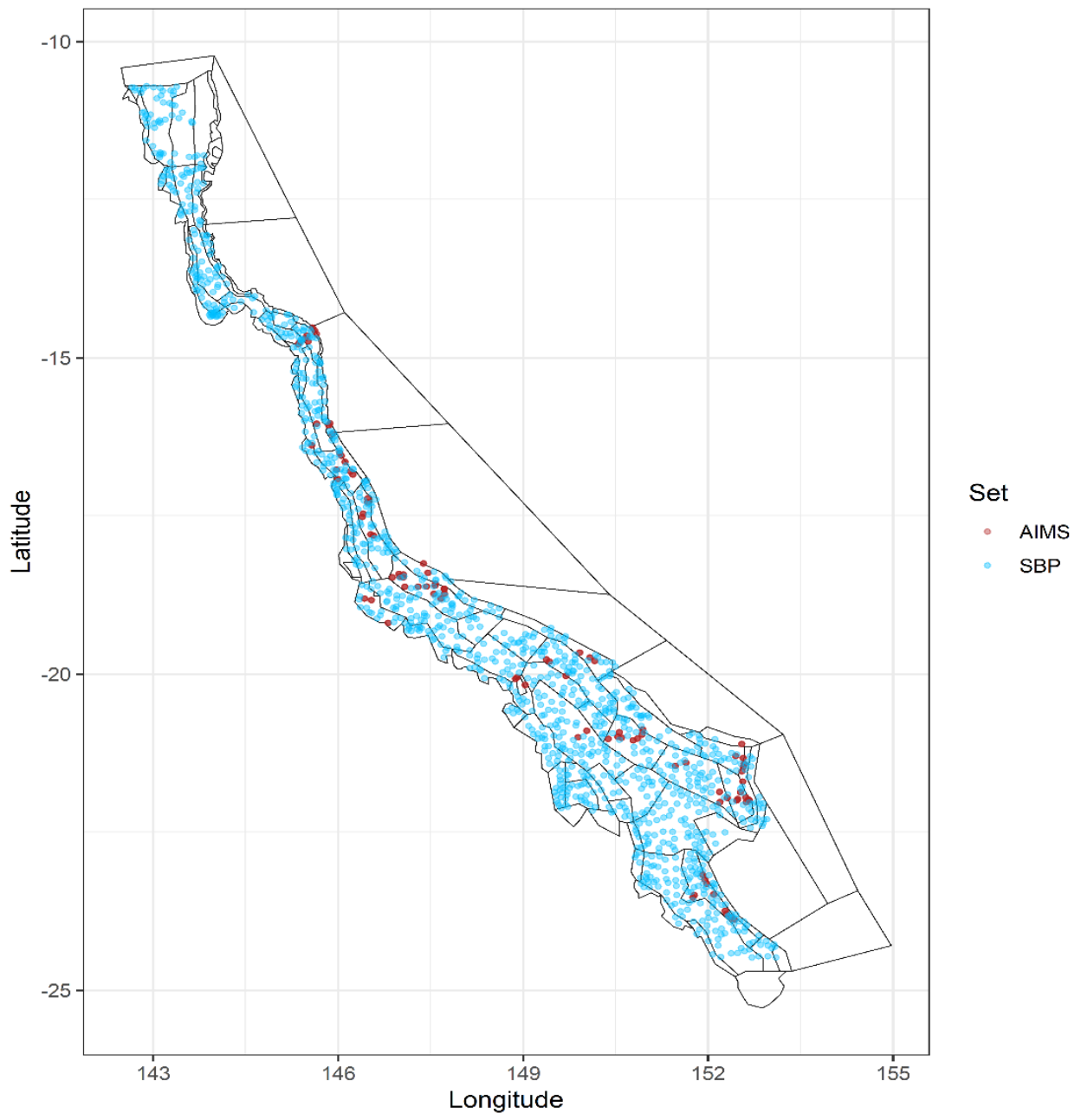

Figure 6.2. Data points from the Seabed Biodiversity Project (blue), and from the AIMS Longterm Monitoring Program (red), overlaid to the model domain of Atlantis Great Barrier Reef. 

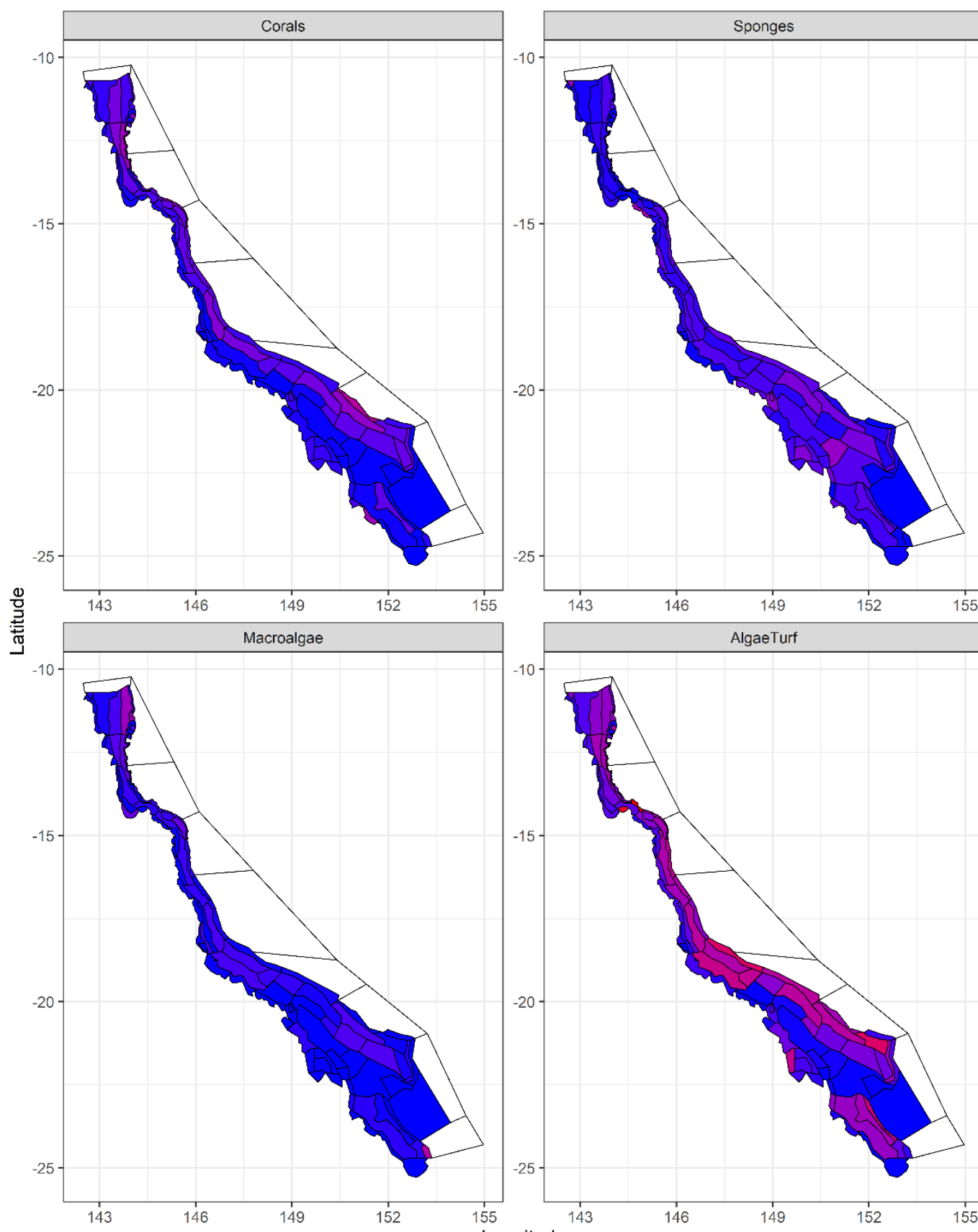

Cover (0-1)
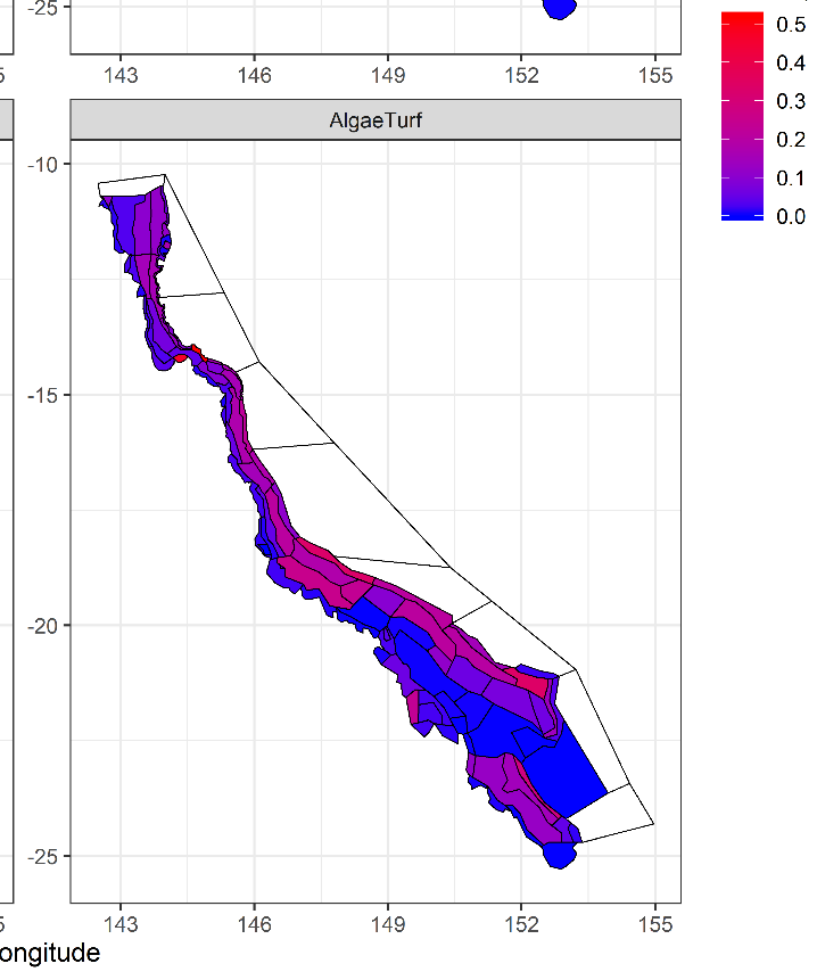

Figure 6.3. Proportional cover of the main benthic groups in Atlantis Great Barrier Reef, as estimated by combining data from the Seabed Biodiversity Program (Pitcher et al. 2007) and the Long-term Monitoring Program from AIMS. Values indicate the 0-1 proportion of a box occupied by the group. White boxes are non-dynamic boundary boxes. 


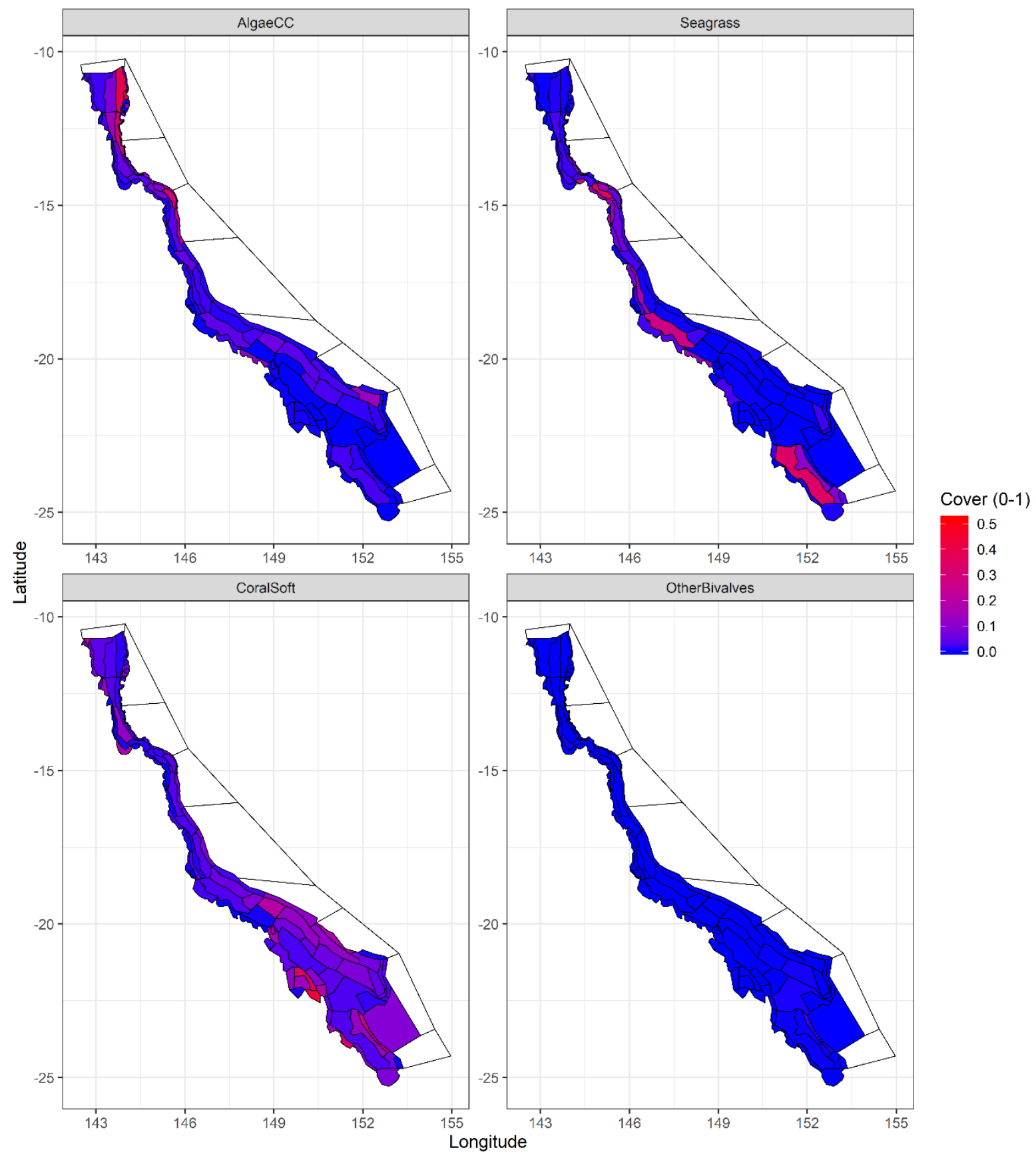

Figure 6.3. Continued. 


\subsubsection{Repartition of total sponge cover among 3 sponge model groups}

Sponges in Atlantis GBR were modelled as 3 groups, based on their trophic mode and on whether they bioerode carbonates: heterotrophic, phototrophic, and bioeroding. However, both SBP and AIMS LTMP datasets reported percentage cover of all sponges combined, and the values of sponge cover had to be redistributed among the 3 sponge groups. While biomass data collected as part of the SBP have high taxonomic resolution and have been used to estimate the distribution of the most abundant sponge taxa on the GBR (Sutcliffe et al. 2010), this dataset did not include shallow coral reefs (Pitcher et al. 2007). Information on the proportion of bioeroding sponges on GBR sponge assemblages was found only for inner reefs (Ramsby et al. 2017). Furthermore, no other large-scale dataset was available on the relative abundance of heterotrophic and phototrophic sponges on the GBR, partly because the importance of phototrophy in the diet of many sponge species has never been quantified.

Previous studies have shown that proportions of the two trophic types (heterotrophic and autotrophic) in GBR sponge assemblages change with distance from shore. In general, heterotrophic sponges dominate sponge assemblages on inshore reefs, whereas upwards of $50 \%$ of sponges on offshore reefs are phototrophic (Wilkinson and Cheshire 1989, 1990). This gradient in the composition of sponge assemblages across the continental shelf of the GBR is has been attributed to increasing water quality the further offshore (Wilkinson 1987, Wilkinson and Cheshire 1989, 1990). Namely, higher concentrations of organic nutrients and higher suspended sediments support heterotrophic feeding and limit phototrophy on inner reefs, whereas clear and nutrient-poor offshore waters promote phototrophic feeding on outer reefs (Wilkinson 1987). However, opposite trends have been shown more recently (Bannister et al. 2010), showing that heterotrophic sponges can be more abundant on outer reefs than on inner reefs. Proportions of bioeroding sponges also vary with distance from shore on the GBR, with inshore reefs presenting in general higher abundance of bioeroding sponges than offshore reefs (Sammarco and Risk 1990). Clionid bioeroding sponges on inshore reefs on the GBR can make up for $50 \%$ of total sponge cover (mean 10-20\%), but spatial heterogeneity between reefs is high, and bioeroding sponges are virtually absent from some reefs (Ramsby et al. 2017). Data for proportion of bioeroding sponges on outer reefs were not available at the time of this Thesis.

To date, there is limited information on the variability of sponge assemblages across latitudes on the GBR, especially on coral reefs. Sled and trawl data from Pitcher et al. (2007) showed no obvious variation in biomass of the main sponge species across latitudes, with distribution 
of the most abundant sponge species being continuous across a latitudinal gradient (Sutcliffe et al. 2010). In addition, George et al. (2018) found limited variability in sponge morphology on the N-S axis of the GBR.

Because no regional-scale data was available, and based on existing information, rough estimates for proportions of heterotrophic, phototrophic, and bioeroding sponges in model boxes of Atlantis GBR were derived for coastal boxes, mid-shelf boxes, and offshore boxes, and were assumed to be constant across latitudes. Sponge proportional cover in each box was multiplied by a vector $v=\left[P_{h e t}, P_{p h o}, P_{b i o}\right]$, where $P_{h e t}$ is the proportion of heterotrophic sponges, $P_{p h o}$ of phototrophic, and $P_{b i o}$ of bioeroding sponges in the box, and such that $P_{h e t}+P_{p h o}+P_{b i o}$ $=1$. It was assumed that bioeroding sponges were absent from sandy and muddy habitats. Sponge cover in different model boxes obtained from the SBP and AIMS datasets was multiplied by the following vectors:

- coastal boxes / inner reefs: [0.6, 0.2, 0.2] for heterotrophic, phototrophic, and bioeroding, respectively

- mid-shelf boxes: $[0.45,0.45,0.1]$ for heterotrophic, phototrophic, and bioeroding, respectively

- offshore boxes / outer reefs: [0.4, 0.5, 0.1] for heterotrophic, phototrophic, and bioeroding, respectively

- boxes with no reef cover: $[0.5,0.5]$ for heterotrophic and phototrophic, respectively

Values of sponge cover used in as initial conditions in Atlantis GBR are shown in Fig. 6.4 (note the values refer to the proportional cover based on the area of the entire box). Because sponge assemblages on the GBR show large variability even at small spatial scales (Wilkinson and Evans 1989, Wörheide et al. 2005), and because most studies reported proportions of the trophic types in GBR sponge assemblages as sponge biomass (except Ramsby et al. 2017), the approach used here has limitations. While this procedure provided the best attainable estimates of the proportions of sponge cover for the scope of this Thesis, data quantifying abundance and distribution of sponge trophic groups across the GBR should be used to validate and correct this implementation as they become available. 


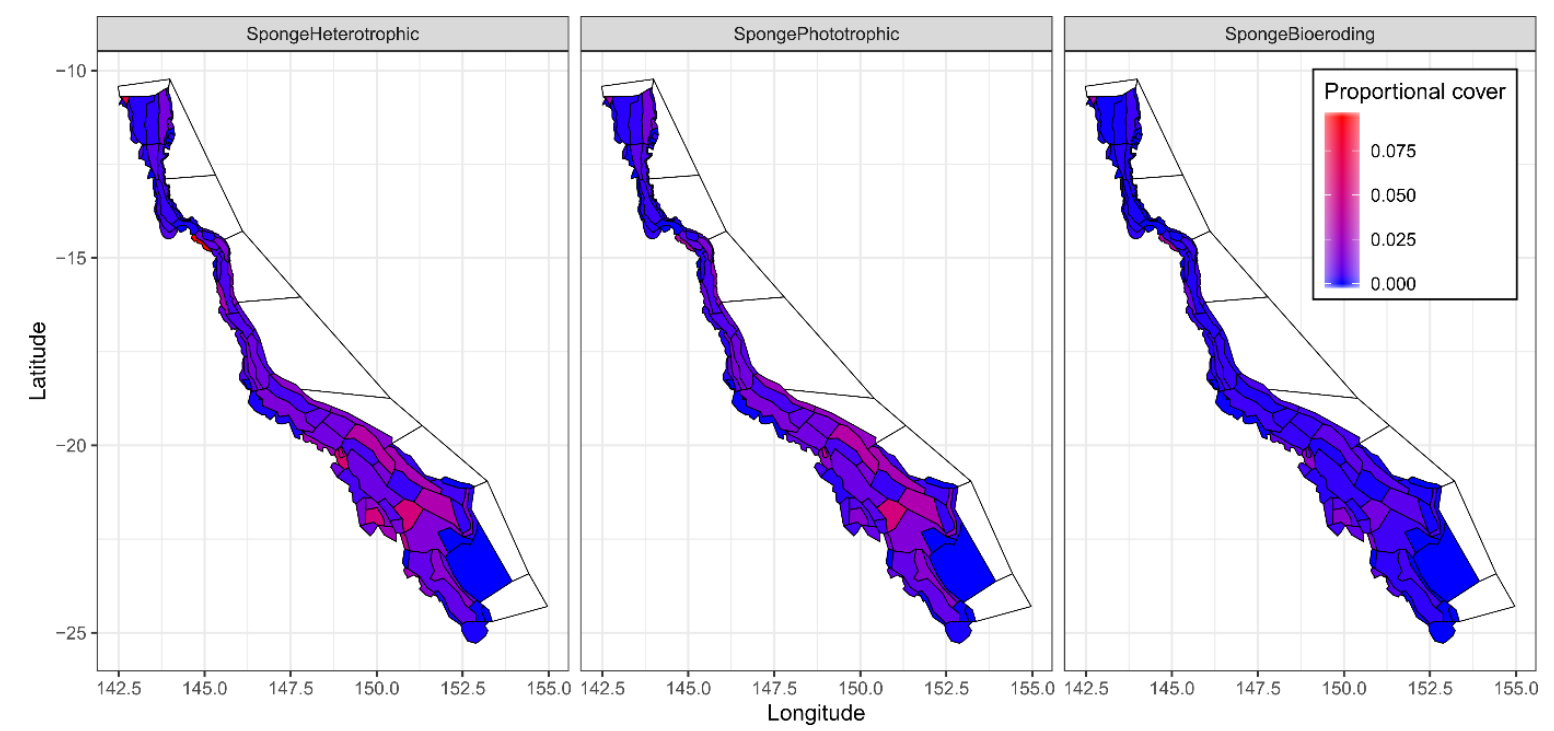

Figure 6.4. Proportional cover of heterotrophic, phototrophic, and bioeroding sponge in Atlantis Great Barrier Reef. Values refer to fraction of total box area occupied by a group. White boxes indicate non-dynamic boundary boxes.

\subsubsection{Biomass of benthic groups}

Initial conditions for Atlantis also require estimates of the biomass of all functional groups in all model boxes, expressed as $\mathrm{mg} \mathrm{N} \mathrm{m}^{-2}$ for epibenthic groups (Audzijonyte et al. 2019). Biomass of benthic organisms is often impractical to measure, and collecting biomass samples may damage or kill the organisms (see Bell et al. 2017). Studies reporting biomass of benthic organisms are rare, especially on coral reefs where destructive sampling methods often cannot be utilised. In the scope of the SBP, benthic organisms were sampled on the inter-reef seabed with an epibenthic sled and a research trawl, in order to obtain biomass estimates per unit area (Pitcher et al. 2007). SBP biomass data were included in the development of Atlantis GBR for model groups that mostly inhabit inter-reef areas (including benthic detritivores, some echinoderms, and some crustaceans). However, these data could not adequately represent reefdwelling organisms, because coral reefs had not been sampled extensively by the SBP. The AIMS LTMP dataset also did not contain biomass data for benthic organisms. Therefore, biomass for the initial conditions of benthic organisms on coral reefs was estimated based on proportional cover.

Some previous Atlantis applications have used generic estimates of the values of biomass per unit area for all benthic organisms (e.g. $30 \mathrm{~g} \mathrm{C} \mathrm{m-2} \mathrm{in} \mathrm{Weijerman} \mathrm{et} \mathrm{al.} \mathrm{2014,} \mathrm{after} \mathrm{Crossland}$ et al. 1991). Studies reporting values of biomass of benthic organisms per unit area are rare. Moreover, functional groups in Atlantis GBR contained organisms with heterogeneous 
structural properties. Sponge model groups, for example, were based on their trophic mode and potential to bioerode. However, sponges can present high morphological diversity, ranging from 2D encrusting patches to complex 3D growth forms (Boury-Esnault and Rützler 1996), and the values of biomass per unit area of such different sponge morphologies are likely different. Due to these limitations, a theoretical maximum biomass $\left(B_{\max }\right)$ per unit area $(\mathrm{mg} \mathrm{N}$ $\mathrm{m}^{-2}$ ) was obtained from published sources for each benthic group, and scaled by the proportional cover to estimate biomass. Because Atlantis assumes that conditions inside a box are homogeneous (Audzijonyte et al. 2019), proportional cover of a benthic organism $X$ in a model box $Y$ is equal to its proportional cover per unit area $\left(1 \mathrm{~m}^{2}\right)$. Biomass $B_{X Y}\left(\mathrm{mg} \mathrm{N} \mathrm{m}^{-2}\right)$ for a benthic group $X$ in a box $Y$ was calculated as $B_{X Y}=B_{\max X} C_{X Y}$, where $B_{\max X}$ is the theoretical maximum biomass per unit area $\left(\mathrm{mg} \mathrm{N} \mathrm{m}^{-2}\right)$ in conditions of $100 \%$ cover of $X$, and $C_{X Y}$ is the proportional cover of $X$ in box $Y$. A number of sources and assumptions were used to estimate $B_{\text {max }}$ for the benthic groups in Atlantis GBR (Tab. 6.2). Conversion factors collated by Brey et al. (2010) for the closest taxon were used to convert other units of biomass to $\mathrm{mg} \mathrm{N} \mathrm{m}^{-2}$.

Table 6.2. Values of maximum biomass $\mathrm{B}_{\max }$ of the benthic groups in Atlantis Great Barrier Reef, the assumptions used to derive them, and the sources of the data utilised.

\begin{tabular}{|c|c|c|c|}
\hline Group & $\begin{array}{c}B_{\max }(\mathbf{m g} \mathbf{N} \\
\left.\mathbf{m}^{-2}\right)\end{array}$ & Assumptions and notes & Source \\
\hline $\begin{array}{l}\text { Massive } \\
\text { corals }\end{array}$ & 3077 & $\begin{array}{l}\text { Based on tissue biomass }\left(\mathrm{mg} \mathrm{cm}^{-2}\right) \text { for Porites and } \\
\text { Goniastrea (Edmunds et al. 2014) }\end{array}$ & \multirow{3}{*}{$\begin{array}{l}\text { Brey et al. (2010); } \\
\text { Edmunds et al. } \\
\text { (2014) }\end{array}$} \\
\hline $\begin{array}{l}\text { Branching } \\
\text { corals }\end{array}$ & 643 & $\begin{array}{l}\text { Based on tissue biomass }\left(\mathrm{mg} \mathrm{cm}^{-2}\right) \text { for Acropora } \\
\text { and Pocillopora (Edmunds et al. 2014) }\end{array}$ & \\
\hline $\begin{array}{l}\text { Encrusting } \\
\text { corals }\end{array}$ & 1625 & $\begin{array}{l}\text { Based on tissue biomass }\left(\mathrm{mg} \mathrm{cm}^{-2}\right) \text { for Montipora } \\
\text { and Orbicella (Edmunds et al. 2014) }\end{array}$ & \\
\hline $\begin{array}{l}\text { Sponges } \\
\text { bioeroding }\end{array}$ & 1488 & $\begin{array}{l}\text { From tissue biomass ( } \mathrm{mg} \mathrm{WW} \mathrm{cm}^{-2} \text { ) of Cliona } \\
\text { orientalis (Fang et al. 2013). Encrusting } C \text {. } \\
\text { orientalis assumed to be } 1 \mathrm{~cm} \text { thick. Value chosen } \\
\text { because: it was standardised to unit area occupied } \\
\text { only by the sponge; and because uniform sponge } \\
\text { thickness allowed easy scaling to } \mathrm{m}^{2}\end{array}$ & $\begin{array}{l}\text { Wilkinson (1987); } \\
\text { Opitz (1996); } \\
\text { Brey et al. (2010); } \\
\text { Fang et al. } 2013\end{array}$ \\
\hline $\begin{array}{l}\text { Sponges } \\
\text { phototrophic }\end{array}$ & 2976 & $\begin{array}{l}\text { Approximated as } B_{\max } \text { of bioeroding sponge x } 2 \text {, to } \\
\text { account for thicker sponge body plan }(\sim 2 \mathrm{~cm}) \text {. } \\
\text { Validated against values of sponge biomass on } \\
\text { Caribbean reefs (Opitz 1996) and the GBR } \\
\text { (Wilkinson 1987) }\end{array}$ & $\begin{array}{l}\text { Wilkinson (1987); } \\
\text { Opitz (1996); } \\
\text { Brey et al. (2010); } \\
\text { Fang et al. (2013) }\end{array}$ \\
\hline
\end{tabular}




\begin{tabular}{|c|c|c|c|}
\hline Group & $\begin{array}{c}B_{\max }(\mathbf{m g ~ N} \\
\left.\mathbf{m}^{-2}\right)\end{array}$ & Assumptions and notes & Source \\
\hline $\begin{array}{l}\text { Sponges } \\
\text { heterotrophic }\end{array}$ & 5952 & $\begin{array}{l}\text { Approximated as } B_{\max } \text { of bioeroding sponge } \mathrm{x} 4 \text {, to } \\
\text { account for thicker sponge body plan }(\sim 4 \mathrm{~cm}) \text {. } \\
\text { Validated against values of sponge biomass on } \\
\text { Caribbean reefs (Opitz 1996) and the GBR } \\
\text { (Wilkinson 1987) }\end{array}$ & $\begin{array}{l}\text { Wilkinson (1987); } \\
\text { Opitz (1996); } \\
\text { Brey et al. (2010); } \\
\text { Fang et al. } 2013\end{array}$ \\
\hline Turf algae & 7520 & $\begin{array}{l}\text { From dry weight (mg cm-2) of epilithic filamentous } \\
\text { algae (De Ruyter van Stenevick and Breeman, } \\
\text { 1981) }\end{array}$ & $\begin{array}{l}\text { De Ruyter van } \\
\text { Stenevick and } \\
\text { Breeman (1981); } \\
\text { Klumpp and } \\
\text { McKinnon (1989); } \\
\text { Opitz (1996) } \\
\text { Brey et al. (2010) }\end{array}$ \\
\hline Macroalgae & 7520 & $\begin{array}{l}\text { From dry weight (mg cm-2) of epilithic filamentous } \\
\text { algae (De Ruyter van Stenevick and Breeman, } \\
\text { 1981), validated against similar values of biomass } \\
\text { of the algal community on the GBR (Klump and } \\
\text { McKinnon, 1989) }\end{array}$ & $\begin{array}{l}\text { De Ruyter van } \\
\text { Stenevick and } \\
\text { Breeman (1981); } \\
\text { Klumpp and } \\
\text { McKinnon (1989); } \\
\text { Opitz (1996); } \\
\text { Brey et al. (2010); }\end{array}$ \\
\hline CCA & 3826 & $\begin{array}{l}\text { From dry weight (mg cm-2) of Hydrolithon } \\
\text { boergesenii and Litophyllum cf. intermedium (De } \\
\text { Ruyter van Stenevick and Breeman, 1981) }\end{array}$ & $\begin{array}{l}\text { De Ruyter van } \\
\text { Stenevick and } \\
\text { Breeman (1981); } \\
\text { Brey et al. (2010) }\end{array}$ \\
\hline Soft corals & 1663 & $\begin{array}{l}\text { From tissue biomass (g WW) of Dendronephtya sp. } \\
\text { (Harder et al. 2003), similar to tissue biomass (g } \\
\text { DW) of Sarcophyton sp. in Sella and Benayahu } \\
\text { (2010) }\end{array}$ & $\begin{array}{l}\text { Harder et al. (2003); } \\
\text { Brey et al. (2010); } \\
\text { Sella and Benayahu } \\
\text { (2010) }\end{array}$ \\
\hline Seagrass & - & Dynamic model (see above) & Baird et al. (2016) \\
\hline Others & 5236 & $\begin{array}{l}\text { Equal to } \sim 30 \mathrm{~g} \mathrm{C} \mathrm{m}^{-2} \text { for invertebrates (Brey et al. } \\
\text { 2010), value used in Atlantis Guam as generic } \\
\text { estimate of benthos biomass (Weijerman et al. } \\
\text { 2014). }\end{array}$ & $\begin{array}{l}\text { Crossland et al. } \\
\text { (1991); } \\
\text { Brey et al. (2010); } \\
\text { Weijerman et al. } \\
\text { (2014) }\end{array}$ \\
\hline
\end{tabular}




\subsubsection{Spongivorous fishes}

Predation from spongivorous fishes is an important driver of sponge assemblages in the Caribbean (Pawlik et al. 2018), whereas the intensity of fish-sponge trophic interactions in the Indo-Pacific are less clear. Reports of spongivorous vertebrates from the GBR are very limited, with records of sponge biomass in the gut contents of rabbitfishes (Pitt 1997, Hoey et al. 2013), and of hawksbill turtles selectively grazing sponges (Meylan 1988). In the Indo-Pacific, butterflyfishes have been observed to feed on sponges (Sano et al. 1989, Nagelkerken et al. 2009), as well as other reef fishes like Zanclus cornutus (Powell et al. 2015) and angelfishes (Charlotte Mortimer pers. comm.). However, while the diet of many omnivorous reef fishes includes sponges, some fish species specialise in sponge consumption (Wulff 2006a). These specialised sponge feeders may be directly impacted by changes in sponge dominance on future reefs (see Chapter 2, Bell et al. 2018a). Because Atlantis is, at its core, a food web model, these direct trophic interactions can have a significant impact on model dynamics. For this reason, it was desirable to add one trophic group of spongivorous fishes to Atlantis GBR.

In earlier stages of model development of Atlantis GBR, sponges were included in the diet of fish groups like omnivorous reef fish, small reef fishes, and scraping herbivores. GBR-wide data on the abundance and distribution of spongivorous fishes on the GBR was not available at the time of this thesis. For this reason, one generic group of specialised spongivorous fishes (spongivores hereafter) was included into Atlantis GBR, and assigned a value of biomass in the initial conditions equal to $1 / 2$ the biomass of corallivorous fishes. This value was arbitrary, and its choice was due to the absence of data. Spongivores were assumed to prefer reef habitat. Biomass of different age-classes of spongivorous fishes (fish groups in Atlantis are agestructured), and their biomass and abundance in each model box, were estimated based on life history traits of species of fishes that have been reported to feed on sponges. These included Pomacanthus sextriatus, Siganus puellus, and Chaetodon kleinii, and traits of these species to be used in the model were retrieved from Fishbase (Froese and Pauly 2019).

\subsubsection{Parametrisation of sponge groups}

Model parameters for the 3 sponge groups and the group of spongivorous fishes were obtained from published sources. For the purposes of the parametrization, sponge groups were parameterised based on the most common, abundant, and well-studied coral reef sponges on the GBR. Bennett et al. (2017) evaluated the effects of OW and OA on 4 abundant GBR sponge species: heterotrophic Stylissa flabelliformis and Rhopaloeides odorabile; and phototrophic 
Cymbastela coralliophila and Carteriospongia foliascens. Therefore, information on traits of these species was used to parameterise sponge groups in Atlantis GBR. Online databases like Scopus (www.scopus.com) and Web of Science (https://apps.webofknowledge.com) were searched for studies on these species on the GBR. When no studies were found for a certain trait, studies on different sponge species and/or from different regions were used.

Atlantis has a lot of parameters, many of which are specific to Atlantis formulations (Audzijonyte et al. 2019). For this reason, reporting values of model parameters here would not be useful to non-Atlantis users. Sources of the parameters that represent main sponge traits in Atlantis GBR are reported in Table 6.3.

Table 6.3. Main traits of sponge groups in Atlantis Great Barrier Reef, examples of the corresponding Atlantis parameters (see Audzijonyte et al. 2019 for the function of each parameter), and the sources used to parameterise them.

\begin{tabular}{|c|c|c|}
\hline Trait & $\begin{array}{l}\text { Atlantis parameters } \\
\text { (see Audzijonyte et al. } \\
\text { 2019) }\end{array}$ & Source \\
\hline Effects of OW and OA & $\begin{array}{l}\text { TCorr, phCorr, q10 } \\
\text { (physiological } \\
\text { parameters) }\end{array}$ & $\begin{array}{l}\text { Griffith et al. (2011), Bennett } \\
\text { et al. (2017, 2018), Ramsby et } \\
\text { al. (2018) }\end{array}$ \\
\hline Pumping and feeding & $\mathrm{C}$ (clearance rates) & Massaro et al. (2012) \\
\hline Assimilation efficiency & $\begin{array}{l}\text { E, EPlant, EDL, EDR } \\
\text { (proportions } \\
\text { of } \\
\text { assimilated food) }\end{array}$ & $\begin{array}{l}\text { De Goeij et al. (2009), } \\
\text { Koopmans et al. (2010) }\end{array}$ \\
\hline $\begin{array}{l}\text { Respiration and oxygen } \\
\text { consumption }\end{array}$ & $\begin{array}{l}\mathrm{KO} 2, \mathrm{mO} \text { (aerobic } \\
\text { metabolism and response } \\
\text { to anoxia) }\end{array}$ & Mills et al. (2014) \\
\hline $\begin{array}{l}\text { Production of ammonia and } \\
\text { detritus }\end{array}$ & $\begin{array}{l}\text { FDG, FDGDL, FDGDR } \\
\text { (proportion of egestion } \\
\text { and excretion) }\end{array}$ & $\begin{array}{l}\text { De Goeij et al. (2009), Hadas } \\
\text { et al. (2009), Maldonado et al. } \\
\text { (2012) }\end{array}$ \\
\hline
\end{tabular}




\begin{tabular}{|c|c|c|}
\hline Growth rates & $\begin{array}{l}\text { mum (maximum growth } \\
\text { rate) }\end{array}$ & $\begin{array}{l}\text { Duckworth et al. (2012), } \\
\text { Abdul Wahab (2014) }\end{array}$ \\
\hline Diet and food items & Prey availability matrix & $\begin{array}{l}\text { Reiswig (1971a, 1971b), de } \\
\text { Goeij and van Duyl (2007), de } \\
\text { Goeij et al. (2008), Topcu et } \\
\text { al. (2010), Mueller et al. } \\
\text { (2014), McMurray et al. } \\
\text { (2018) }\end{array}$ \\
\hline Reproduction and recruitment & $\begin{array}{l}\text { KDENR, Reca, Recb } \\
\text { (parameters of stock- } \\
\text { recruitment relationships) }\end{array}$ & $\begin{array}{l}\text { Abdul Wahab et al. (2014a, b, } \\
\text { c, d), Whalan et al. (2015) }\end{array}$ \\
\hline
\end{tabular}

\subsubsection{Model calibration}

After all model components (such as model domain, physical forcings, and initial conditions) have been specified, Atlantis models need to be calibrated. Calibration in Atlantis is an iterative process of modifying model parameters until the model is stabilised. A typical approach is to consider the model stable when, at the end of the simulation run, the biomass of all groups is roughly equal to their biomass in the initial conditions $\pm 20 \%$ (Audzijonyte et al. 2019). Calibration of Atlantis models focuses most often on growth, mortality, and consumption (clearance) rates, and on the intensity of predator-prey interactions.

The calibration of an Atlantis model can be a time-consuming process. Because model runs require analysis in order to plan how to change parameters appropriately, and because parameters are changed manually, model calibration is typically difficult to automate. Atlantis GBR is a large model, and simulations can take a long time to run on desktop and laptop computers (typically, a 50-year run can take approx. 10-20 hours, depending on the hardware). Atlantis GBR is currently undergoing model calibration, and results of this process will be reported in the future. 
Chapter 7. General Discussion 


\subsection{Summary of key findings}

This thesis investigated aspects of the development of sponge assemblages over time, and some of the ecosystem-level effects of a shift towards sponge dominance on future coral reefs. This thesis had five main aims: (1) Chapter 2 aimed to qualitatively predict the ecosystemlevels effects of a perturbation-driven shift towards sponge dominance on coral reefs, in order to elucidate the ecological function of transitioning reefs;(2) Chapter 3 aimed to quantify the temporal variability of sponge abundance and biodiversity at an Indonesian coral reef, in order to understand how sponge assemblages can develop over time; (3) Chapter 4 aimed to characterise temporal dynamics and the role of available substrate on the same Indonesian reef, in order to elucidate how communities develop when colonizable substrate becomes available; (4) Chapter 5 aimed to determine whether sponge-dominated reefs are as structurally complex as coral reefs, in order to understand how reef architecture will change on transitioning reefs; and finally, (5) Chapter 6 aimed to draw from the results of the qualitative modelling in Chapter 2 and from the field studies in Chapters 3, 4, and 5 to guide the development of a framework to model sponges in the end-to-end ecosystem model Atlantis. The key findings of this thesis were: (1) sponge-dominated reefs may be low-complexity, high-detritus ecosystems, but their functioning will depend on the ubiquity of the sponge loop, the outcomes of competitive interactions with macroalgae, and the role of sponges as providers of habitat; (2) sponge abundance and biodiversity can show large temporal variability, with potential consequences for sponge functional roles that depend on sponge population dynamics; (3) percentage cover of sponges and turf algae can show long-term stability and high interannual variability, and only turf algae directly benefit from the opening of colonizable substrate; (4) upright sponge morphologies can provide reef rugosity and large-scale complexity comparably to corals, but the loss of branching corals will correspond to reduced small-scale structural complexity; and (5) ecological models of coral reefs should capture sponges with a degree of detail comparable to that of corals.

In this chapter, I discuss my results in the broader context of sponge ecology on coral reefs, and I consider the limitations and consequences of regime shifts towards sponge reefs. In addition, here I discuss the reasons and advantages for sponges to be incorporated into ecosystem models for coral reefs. Finally, I draw on the results of this thesis to discuss future avenues for research on regime shifts of coral reef to sponge reefs. 


\subsection{Sponge temporal variability: limitations to increases in sponge abundance}

In Chapters 3 and 4 I showed that sponge abundance, diversity, and percentage cover on coral reefs can show strong temporal variability over short time scales (less than a decade). This suggests that sponge assemblages have the potential to rapidly develop by increasing in abundance in response to free substrate and other resources becoming available on the reef. The temporal variability in sponge abundance is important in the context of regime shifts because sponge-mediated functional roles are coupled with sponge demographics (McMurray et al. 2017). However, the increase in sponge abundance on transitioning coral reefs may be limited by many factors, physical and biotic.

\subsubsection{Physical limitations to sponge dominance}

Model QM1 from Chapter 2 assumed that increased water temperature has a negative effect on coral reef sponges, whereas increased acidity and turbidity impact sponges negligibly compared to corals. While this simplification was necessary for model formulation, experimental studies have shown that many sponge species can tolerate future conditions of environmental change (see references in Chapter 1). In particular, ocean acidification (OA) alone appears to have limited effects on sponges (Duckworth and Peterson 2012, Duckworth et al. 2012, Wisshak et al. 2013, Vicente et al. 2016, Bennett et al. 2017). In addition, high $p \mathrm{CO}_{2}$ has been found to mitigate the effects of warming on some abundant phototrophic Great Barrier Reef (GBR) sponges (Bennett et al. 2017), and experiments suggested that bioeroding sponges will excavate carbonates more easily under OA conditions (Wisshak et al. 2012, Fang et al. 2013, Wisshak et al. 2013, 2014, Stubler et al. 2015). I did not detect any effect of sea surface temperature (SST) and SST anomalies over the period 2005-2017 on sponge abundance and population growth of the Indonesian sponge assemblage studied in Chapter 3. This lack of effect of temperature on sponge population dynamics may have been due to relative stability of SST at the study location over the study period. Moreover, a spatial and temporal mismatch between sponge abundance data and SST data (see Chapter 3, Rovellini et al. 2019) may have impeded the detection of weak effects of temperature on the sponge assemblage. However, increased temperature has been shown to have negative effects on sponge physiology (Wisshak et al. 2013, Bennett et al. 2017, 2018, see references in Chapter 1), and some studies have demonstrated that projected levels of ocean warming (OW) will have negative effects on sponge metabolism and decrease sponge survival (Fang et al. 2014, Ramsby et al. 2018). Furthermore, combined OW and OA have negative effects on GBR heterotrophic sponges 
(Bennett et al. 2017), and on and the microbiome associated with the barrel sponge Xestospongia muta in the Caribbean (Lesser et al. 2016).

Reef degradation due to pollution is also affecting coral reefs globally. Decreased water quality and a corresponding increase in suspended sediments can have contrasting impacts on coral reef sponges (Bell et al. 2015b). High sponge abundance on Caribbean coral reefs is partly attributed to the large input of DOC into the Gulf of Mexico from riverine input and from desert dust (Pawlik et al. 2016), and bioeroding sponges in the Caribbean benefit from sources of sewage (Chaves-Fonnegra et al. 2007). Conversely, heterotrophic sponges on the GBR were found to be more abundant on mid-shelf and outer reefs compared to inshore reefs, possibly as a result of decreased water quality and increased sedimentation (Bannister et al. 2010). Responses of sponges to sedimentation in the Indo-Pacific are variable. For example, the phototrophic sponge Lamellodysidea herbacea has shown potential for photoacclimation and overall adaptability to sedimented environments in the Wakatobi (Biggerstaff et al. 2015, 2017a). At the degraded site of Biggerstaff et al. (2015, 2017a, b), high sedimentation rates limit coral growth (Crabbe and Smith 2005). The increase in sponge abundance at this degraded site corresponds to a decrease in colonizable space for corals, thus representing a negative feedback that, together with high sedimentation, may cause the establishment of an alternative sponge-dominated state (see Norström et al. 2009). However, a long-term increase in sponge abundance and percentage cover was not observed at Buoy 3 between 2005 and 2017 (Chapters 3 and 4). The vertical aspect of the Buoy 3 reef, and the lower human impact at Buoy 3 compared to the degraded site from Biggerstaff et al. (2015, 2017a, b), contribute to lower sedimentation rates at Buoy 3 (see Marlow et al. 2018d). The lack of prolonged sedimentation and of widespread temperature-driven coral mortality, and the general stability of the benthic community, have not allowed the establishment of the ecological feedbacks that are necessary for a shift to a sponge-dominated state to follow to a momentary increase in sponge abundance.

While some sponges can adapt to sedimented environments, sedimentation can have negative effects on Indo-Pacific sponges. For example, there are metabolic costs associated with the exposure to high sedimentation in the barrel sponge Xestospongia testudinaria (McGrath et al. 2017). Similarly, while Indo-Pacific Clionid bioeroding sponges were found capable of photoacclimating to low-light conditions associated with turbid environments (Marlow et al. 2018d), their bioerosion rates are limited by sedimentation, also likely due to the metabolic costs of coping with settled sediments (Marlow et al. 2018c). 
In summary, increases in abundance of some sponge species will likely be limited by a number of physical factors, whereas other species will be more suited to tolerate the change or adapt to it. Therefore, the response of coral reef sponges to global climate change and the decrease in water quality will likely vary across reef systems, and may be species-specific depending on trophic mode and acclimation potential of different sponge species.

\subsubsection{Competitive limitations to sponge dominance}

In Chapter 2, all models predicted an ambiguous response of macroalgae to an increase in sponge abundance. This outcome is consistent with the limited knowledge of the interactions between sponges and algae on coral reefs, and in particular the unknown effect of increased sponge abundance on algae. Transitions towards algal dominance are the most widely documented regime shifts for coral reefs, and Chapter 4 showed that filamentous turf algae have a direct and immediate benefit from the opening of free space on the reef. Competition between sponges and macroalgae often shows competitive superiority of algae (see references in Chapter 4). In addition, while sponges can form associations with filamentous turf algae (see Chapter 4), the results of competitive interactions between these have never been explored. It is therefore likely that future shifts towards sponge dominance will be limited by the competitive pressure of macroalgae on sponges.

Increased algal dominance can impede increases in sponge abundance as algae pre-empt available substrate (González-Rivero et al. 2016) and actively displace sponges (Easson et al. 2014). Shifts towards macroalgal dominance on degraded reefs have been observed for decades globally, from the Caribbean (Hughes 1994, Mumby et al. 2007) to the Indo-Pacific (Bruno et al. 2009, Jouffray et al. 2015). However, Indo-Pacific reefs are generally considered less prone to transitioning into states of macroalgal dominance than reefs in the Caribbean (Roff and Mumby 2012), and the majority of Indo-Pacific reefs are still dominated by structure-building corals despite the massive bleaching events of the recent decades (Darling et al. 2019). The lower proportion of Indo-Pacific reefs transitioning towards an algal-dominated state compared to Caribbean reefs is likely due to a number of factors contributing to higher reef resilience on Indo-Pacific reefs. These factors include: lower recruitment rates for macroalgae; lower nutrient in the water columns; higher herbivorous biomass; and higher abundance of branching corals (Roff and Mumby 2012, Darling et al. 2019).

Macroalgae have not been increasing on all coral reefs. In the Wakatobi Marine Natural Park, Indonesia, macroalgae have declined considerably between 2002-2011 (McMellor and Smith 
2010, Curtis-Quick 2013). In addition, macroalgae are typically not abundant on the crests and slopes of many Indo-Pacific reefs (Sawall et al. 2013, Plass-Johnson et al. 2016). At degraded and coral-depleted reefs in the Wakatobi where macroalgae are not abundant, sponges have become the dominant benthic organisms, possibly benefitting from evading competition with algae (Biggerstaff et al. 2017b). Therefore, it is possible that benthic organisms other than algae, including sponges, will benefit from the low algal densities on reef systems that are less prone to transitioning towards macroalgal states, like many Indo-Pacific reefs.

The establishment of positive ecological feedbacks will be necessary for coral reefs to exhibit sponge-dominated alternative stable states. Examples of feedback mechanisms that are needed to maintain a sponge dominated state could be pre-emption of space resulting into decreased recruitment of other benthic organisms, and allelochemical activity resulting into an increase in coral mortality (Norström et al. 2009). In addition, increases in sponge and algal abundance are not mutually exclusive on coral reefs. It has been hypothesised that sponges and algae can be mutually beneficial by exchanging nutrients (Pawlik et al. 2016). Specifically, spongeproduced nutrients are used by macroalgae for primary production, and algal-produced dissolved organic carbon (DOC) is used by sponges for nutrition (Pawlik et al. 2016). This hypothesised 'vicious circle' may be one of the ecological feedbacks behind the low resilience of Caribbean coral reefs, and may be exacerbated by a contemporary increase in turbidity driven by suspended macroalgal exudates, to the further reduction of coral resilience (Mumby and Steneck 2018). However, large quantities of algal-produced DOC on coral reefs also contributes to reef microbialization and related hypoxia (Haas et al. 2016), with unexplored consequences for coral reef sponges. Temporary increases in sponge abundance and interannual changes in sponge percent cover presented in Chapters 3 and 4, respectively, were not followed by a permanent shift towards sponge dominance at Buoy 3. The return of the benthic community at Buoy 3 to a state of lower sponge abundance or percentage cover highlights that: the Buoy 3 reef is resilient to short-term changes in the abundance of benthic organisms, perhaps because this reef has not been impacted by perturbations as much as other sites in the Wakatobi area (Powell et al. 2014, Biggerstaff et al. 2015); no ecological feedback causing a permanent shift in benthic dominance was established at Buoy 3.

In summary, the outcomes of competitive interactions between sponges and algae will likely be species-specific. As a result, respective species compositions of sponge and algal assemblages will likely have an important role in regime shifts towards alternative states on 
coral reefs. More research is needed to elucidate how sponge-algal interactions are resolved in different bioregions.

\subsubsection{Bottom-up biological limitations to sponge-dominance}

Model predictions from Chapter 2 highlighted that sponge dominance on future reefs may be limited by food availability, and higher concentrations of particulate and dissolved organic carbon may be necessary to sustain higher sponge abundance on coral reefs (McMurray et al. 2017). The role of bottom-up limitations in controlling sponge assemblages has been extensively studied in the Caribbean. Previous research showed that sponge abundance and body size in the Caribbean are correlated with the availability of particulate organic carbon (POC) from picoplankton (Lesser 2006, Trussel et al. 2006). However, sponges in the Caribbean feed on both POC and DOC (Reiswig 1971a, McMurray et al. 2016, 2017, 2018), and other authors have suggested that sponges on Caribbean reefs are not food-limited because they can rely on excess DOC from riverine input and wind-borne dust (reviewed in Pawlik et al. 2018). Food limitation structures sponge assemblages in other bioregions, where suspended POC and DOC are not as abundant as on Caribbean reefs. For example, nutrient availability shapes sponge distribution on the GBR, with heterotrophic species dominating sponge assemblages on nutrient-rich inshore reefs, and phototrophic sponges on oligotrophic offshore reefs (Wilkinson and Cheshire, 1989, 1990; but see Bannister et al. 2010 for an inverse pattern likely due to decreased water quality on inshore reefs). Similarly, barrel sponges in the Red Sea are limited by the concentrations of available DOC (Wooster et al. 2019). There is currently a paucity of information for food limitation of Indo-Pacific sponges. It is likely that increases in sponge abundance will be limited by nutrient availability in reef systems where concentrations of POC and DOC are low, and not in systems where they are high, like the Caribbean.

Importantly, DOC consumed by sponges is largely produced by corals and macroalgae (de Goeij et al. 2013, Rix et al. 2017). It is unclear how changes in abundance of corals and algae will affect the DOC pool of coral reefs, but sponges assimilate algal DOC faster than coralproduced DOC (Rix et al. 2017). The affinity of sponges for algal DOC, and the hypothesised mutual benefits between macroalgae and sponges on some coral reefs (Pawlik et al. 2016), suggests that coral decline is unlikely to cause the food limitation of sponge assemblages on transitioning reefs. 


\subsubsection{Top-down biological limitations to sponge dominance}

In the same way that herbivorous fishes are an important control of macroalgae on coraldepleted reefs (Mumby and Steneck 2008), increased sponge dominance on future reefs may be controlled by sponge predators. While top-down structuring of sponge assemblages is wellestablished for Caribbean coral reefs (reviewed in Pawlik et al. 2018), the role of predation as a driver of sponge abundance and diversity is less clear in other regions. Model predictions from Chapter 2 showed that an increase in sponge abundance may be followed by an increase in sponge-eating fishes. However, increased abundance of spongivorous fishes might make shifts towards sponge dominance less likely to happen, particularly on Caribbean reefs, where spongivory is an important driver of sponge assemblage composition (Pawlik et al. 2018, Pawlik and McMurray 2020). Notably, region-wide increases in sponge abundance have been observed over the last few decades on Caribbean reefs (reviewed in Pawlik and McMurray 2020), and the role of top-down limitation to the development of sponge assemblages over time has not been quantified.

In summary, regime shifts of coral reefs towards sponge reefs will likely depend on a broad range of physical and biological controls acting on sponges. It is unlikely that sponges will increase their dominance ubiquitously on coral reefs (Pawlik and McMurray 2020). Furthermore, because different sponge species respond differently to environmental change, increases in sponge dominance will likely be species-specific.

\subsection{Sponge reefs: what could they look like?}

\subsection{1. $\quad$ Ecological functioning of sponge reefs}

The functional roles of sponges largely depend on taxa, trophic mode, and morphology (see Bell 2008). For example, not all sponges are bioeroders, and only upright morphologies contribute to reef complexity (Chapter 5). Therefore, the taxonomic, morphological, and functional composition of future sponge assemblages will be a critical determinant of how sponge reefs will function. In macroalgal-dominated environments, canopy-forming macroalgae can provide habitat for many reef species. However, canopy-forming macroalgae are likely to decline as a result of climate change, with algal assemblages shifting towards the dominance of low-complexity filamentous species (Fulton et al. 2019). Similarly, complex upright sponge morphologies contribute to large-scale structural complexity in a similar way 
to corals (Chapter 5). However, the intensity of storms is predicted to increase with climate change (Emanuel 2013, Cheal et al. 2017), and storms can damage and dislodge upright sponge growth forms (Mercado-Molina and Yoshioka 2009). Furthermore, low-complexity encrusting sponge growth forms have already become dominant on some highly-sedimented reefs (Biggerstaff et al. 2017b). For these reasons, sponge morphological diversity on future reefs may be reduced, leading to lower-complexity environments.

Model results from Chapter 2 showed that, if the sponge loop hypothesis is valid for all coral reefs sponges, detrital pathways will be enhanced on sponge-dominated reefs. However, the sponge loop hypothesis has been tested mostly for Caribbean and Red Sea sponge species, with no information available yet for Indo-Pacific and Australian reefs (Pawlik and McMurray 2020). Furthermore, not all sponges on Caribbean reefs are net producer of detritus (McMurray et al. 2018). While many sponges assimilate large quantities of DOC (although in different proportions, Hoer et al. 2018), cavity-dwelling sponges produce large quantities of cellular detritus (de Goeij et al. 2008, 2009), whereas emergent, upright sponges convert DOC into sponge biomass that is eaten by predators (McMurray et al. 2018). Therefore, dominance of small cavity-dwelling sponge species on future reefs may be associated with enhanced detrital pathways, assuming the ubiquity of the sponge loop hypothesis. The boost of the detrital pools on coral reefs further reduces the potential for corals to recover, due to reduced light availability (Mumby and Steneck 2018). The potential for large temporal variability of small-bodied cryptic sponges (Chapter 3, Rovellini et al. 2019) suggests that these detritus-rich reef systems may be highly unstable. Models have shown that on high-detritus sponge-dominated reefs the productivity of small-bodied, omnivorous, and invertivorous fish will be enhanced as a result of boosted detrital pathways (Silveira et al. 2015). However, the loss of structural complexity on sponge reefs dominated by small-bodied, cavity-dwelling sponges will likely have a negative impact on these fish groups (Chapter 5). The implications for overall reef productivity are therefore unclear.

In contrast, dominance of large-bodied sponge species with upright growth forms may support increased spongivory on reefs where sponge predation is important (McMurray et al. 2018), and the maintenance of coarse-scale structural complexity (Chapter 5). In systems where upright sponge morphologies are abundant, it is likely that increased sponge dominance will support a higher biomass of sponge-eating fishes, similarly to how large macroalgal biomass enhances herbivorous trophic pathways on algal-dominated reefs (see Fulton et al. 2019). However, even on reefs where complex sponge morphologies become abundant, small-scale 
structural complexity will likely be lower on sponge-dominated reefs compared to coraldominated reefs (Chapter 5). As a result, small cryptobenthic fishes that make up for $\sim 50 \%$ of coral reef fish biodiversity (Brandl et al. 2018) and that constitute a key component of coral reef food webs (Brandl et al. 2019) will likely be negatively impacted by the loss of branching corals.

\subsubsection{Sponges to not functionally replace corals}

A transition towards alternative reef states (whether sponge-dominated or not) will cause a loss of the functions carried out by corals. For example, because branching and table corals are the main providers of small-scale structural complexity (Huntington et al. 2017, Chapter 5) and refuge space for reef fish (Shima et al. 2008), the collapse of branching corals will cause a loss of reef complexity regardless of whether a shift towards sponge dominance occurs.

Scleractinian corals are necessary for reef accretion, and it is unlikely that sponges will be able to maintain reef structure long after corals disappear (see Chapter 5). In fact, when sponge bioerosion of carbonates is considered, sponges have an overall negative contribution to reef structure (Pawlik and McMurray 2020). The increase of bioeroding sponges on future coral reefs will likely be limited by warmer seawater (Ramsby et al. 2018) and by competing algae (González-Rivero et al. 2012, 2016, López-Victoria et al. 2006). However, sponge-mediated dissolution of carbonates under increased $\mathrm{OA}$ is expected to increase in the near future (see Schönberg et al. 2017). Therefore, an increase of sponges on future reefs may further accelerate the flattening of coral reefs caused by the ongoing loss of corals (Alvarez-Filip et al. 2009).

\subsection{Modelling regime shifts of coral reefs to sponge reefs}

My qualitative modelling (Chapter 2, Bell et al. 2018a) highlighted the need to capture aspects of sponge ecology in quantitative ecological models for coral reefs. The results from my field studies have provided some valuable information to help parameterise such models. While predicting the future of coral reefs is difficult, due to the inherent complexity of these ecosystems (Chapter 2, Harborne et al. 2017, Bell et al. 2018a), ecological models can provide insights into the functioning of ecosystems under future scenarios. Sponges have been underrepresented in ecosystem models so far, often being modelled as generic "filter-feeders" with several other benthic organisms (e.g. Fulton et al. 2006, Weijerman et al. 2015). This is partly due to of the often-overlooked ecological importance of sponges. However, the many 
functional roles of marine sponges (Bell 2008), and the higher tolerance to environmental change of some sponge species compared to other benthic organisms (Bell et al. 2018b), indicate that they should be captured by ecosystem models for coral reefs. Ecosystem models like Atlantis should include sponges with a minimum realism that allows the distinction between heterotrophic, phototrophic, and bioeroding sponges, because: (1) these groups have different functional roles on coral reefs; and (2) because they respond differently to environmental change (see Chapter 6). The framework to model sponges in Atlantis proposed in Chapter 6 allows for a flexible and dynamic representation of sponges and their functional roles. Results from Chapter 2, 3, 4, and 5 in this thesis identified at least three aspects of sponge ecology that should be captured explicitly and dynamically in ecosystem models, as achieved in Chapter 6:

1. Because sponge functional roles and sponge demographics are coupled (McMurray et al. 2017), it is important to capture the relationships between sponge abundance and sponge-mediated ecological processes. The large temporal variability of sponge abundance and biodiversity described in Chapter 3 indicated that sponges should be modelled as a dynamic component of the ecosystem, because important spongemediated processes are likely to vary over time.

2. Model predictions from Chapter 2, and the observations presented in Chapter 4, showed that considering the competitive interactions between macroalgae and sponges is crucial to understanding the ecosystem-levels effects of a loss of corals. Interactions between two ecologically important groups like sponges and macroalgae can determine model dynamics, and should therefore be modelled explicitly when evaluating future scenarios of coral reefs.

3. Structural complexity is one of the most important ecological functions of coral reefs that must be preserved in the Anthropocene (Darling et al. 2019, Zawada et al. 2019). Some mechanistic models have considered the contribution of corals to rugosity, and its destruction from thermal stress and bioeroders (e.g. Blackwood et al. 2011, Bozec et al. 2015). The crucial importance of structural complexity in supporting coral reef food webs and fishery productivity (Rogers et al. 2014, 2018) justify the effort of representing reef complexity in more complex ecosystem models too. The potential for sponges to contribute to reef structural complexity has been largely overlooked so far. In Chapter 5 I demonstrated that, while some sponge morphologies can provide reef rugosity and larger-scale complexity (60-30 cm refuge holes), small-scale refugia (5-1 
$\mathrm{cm}$ ) will likely be lost with branching corals. For this reason, ecosystem models of coral reefs should account separately for the contributions of corals and sponges to structural complexity, especially when modelling coral-depleted states.

Essentially, the sponge module for Atlantis presented in Chapter 6 allows modelling sponges with the same degree of details that Atlantis already provided for corals (see Weijerman et al. 2014, 2015). Sponges in Atlantis have user-defined responses to ocean warming and acidification (Audzijonyte et al. 2019), and the model can therefore be used to model the effects of climate change on coral reefs in a way that does not overlook the response of sponges as ecologically important benthic organisms.

Atlantis GBR is a large Atlantis application, both in terms of the model domain and of the number of modelled groups and ecological processes. Calibrating Atlantis models can be difficult and time-consuming, and understanding how the ecological processes of interest are conceptualised in the model is an important first step in this process (Pethybridge et al. 2019). In the scope of this thesis, priority was given to the elucidation of some important aspects of sponge ecology on coral reefs, and to the appropriate inclusion of these aspects in Atlantis. Although using Atlantis to predict the response of coral reef sponges on the GBR to climate change was not possible as part of this thesis, future applications of Atlantis will be able to capture the important aspects of sponge ecology that have been overlooked in modelling efforts so far.

\subsection{Future directions}

To better understand how transitions towards sponge dominance may occur on coral reefs, and better predict how sponge-dominated reefs may function, some important topics in sponge ecology require further research.

1. The analysis of model uncertainty in Chapter 2 showed that competitive interactions between sponges and other benthic organisms, in particular algae, will regulate transition towards coral-depleted states. Future research should focus on how abundant sponge and algal taxa compete on coral reefs in different bioregions.

2. In Chapter $5 \mathrm{I}$ explored the role of sponges as providers of structural complexity at intermediate scales. Future research should quantify the differences between the fish assemblages that are associated with sponge-dominated and coral-dominated reef areas along 
a gradient of structural complexity. In addition, future studies should characterise with higher detail the three-dimensional properties of single sponge morphologies, to identify which sponge species or growth forms must be the focus of conservation efforts.

3. High priority should be given to researching the effects that a regime shifts towards sponge dominance may have on the human use of coral reefs. Coral reefs are the world's most productive marine ecosystems, and support the livelihood of millions of people globally. Ecosystem models that include socioeconomic components, like Atlantis, may be used to explore the flow-on consequences of regime shifts on fisheries, the protection of coastlines from storms, and other ecosystem services of coral reefs. My work has contributed a framework to model sponges in Atlantis, which is now equipped to capture the main known aspects of sponge ecology (Chapter 6). As we gain knowledge on the response of sponges to ocean warming and acidification, Atlantis may be used in the future to explore the effects of sponges becoming dominant on coral reefs.

4. Pawlik and McMurray (2020) highlighted that some aspects of the roles that sponges play in biogeochemical cycles and organic carbon pathways on coral reefs are still unclear. In particular, the ubiquity of the sponge loop across oceanic basins should be established. Similarly, the exchange of nutrients between coral reef sponges and macroalgae (Pawlik et al. 2016) and the resulting increase in detrital pools (Mumby and Steneck 2018) have been hypothesised based on evidence mostly from Caribbean reefs, and the applicability of these notions to other reef systems is yet to be determined.

\subsection{Concluding remarks}

In summary, the results presented in this thesis indicate that the ecological functioning of sponge-dominated reefs will be very different from that of coral reefs. Sponge assemblages are dynamic and can develop rapidly over time, but future increases in sponge abundance on coral reefs will largely depend on the ecological interactions between sponges and algae. Spongedominated reefs may offer coarse structural complexity comparably to coral reefs at the scale of $4 \mathrm{~m}^{2}$, but small refugia $(<5 \mathrm{~cm})$ will be lost if branching corals disappear. While increased sponge abundance may enhance the productivity of spongivores, omnivores, and detritivores, this may be limited by the simplification of reef structure resulting from coral loss.

Shifts towards sponge dominance are unlikely to be ubiquitous, and increases in sponge abundance will depend on many physical and biological controls. In addition, it is unlikely that 
all sponge species will become more abundant on future reefs under environmental change, due to species-specific responses to environmental stress and to biological controls. The taxonomic, morphological, and functional composition of future sponge assemblages will determine how these ecosystems will function.

Coral reefs are rapidly changing on a global scale, and many of them are assuming new configurations as a result of unprecedented transformations (Graham et al. 2014, Hughes et al. 2017b). Shifts towards sponge dominance are one potential avenue of change for some coral reefs. Understanding how these new configurations will origin, how they will function, and how they should be managed will be critical to preserve the ecological and socioeconomic value of coral reefs through the Anthropocene. 


\section{References}

Abdul Wahab, M. A. (2014). A holistic approach towards understanding population dynamics of a coral reef sponge. James Cook University (Townsville, Au).

Abdul Wahab, M. A., de Nys, R., Webster, N. S., and Whalan, S. (2014a). Phenology of sexual reproduction in the common coral reef sponge, Carteriospongia foliascens. Coral Reefs, 33(2):381-394. https://doi.org/10.1007/s00338-013-1119-9

Abdul Wahab, M. A., de Nys, R., Abdo, D., Webster, N. S., and Whalan, S. (2014b). The influence of habitat on post-settlement processes, larval production and recruitment in a common coral reef sponge. Journal of Experimental Marine Biology and Ecology, 461:162172. https://doi.org/10.1016/j.jembe.2014.08.006

Abdul Wahab, M. A., de Nys, R., Webster, N. S., and Whalan, S. (2014c). Larval behaviours and their contribution to the distribution of the intertidal coral reef sponge Carteriospongia foliascens. PLoS ONE, 9(5):e98181. https://doi.org/10.1371/journal.pone.0098181

Adjeroud, M., Poisson, E., Peignon, C., Penin, L., and Kayal M (2019). Spatial patterns and short-term changes of coral assemblages along a cross-shelf gradient in the southwestern lagoon of New Caledonia 11:21.

Adjeroud, M., Augustin, D., Galzin, R., and Salvat, B. (2002). Natural disturbances and interannual variability of coral reef communities on the outer slope of Tiahura (Moorea, French Polynesia): 1991 to 1997. Marine Ecology Progress Series, 237: 121-131.

Aerts, L. A. M. (1998). Sponge/coral interactions in Caribbean reefs: Analysis of overgrowth patterns in relation to species identity and cover. Marine Ecology Progress Series, 175: 241249.

Ainsworth, C., Schirripa, M. J., and Morzaria-Luna, H. (2015). An Atlantis ecosystem model for the Gulf of Mexico supporting Integrated Ecosystem Assessment. NOAA Technical Memorandum NMFS-SEFSC-676 (April).

Alexander, B. E., Liebrand, K., Osinga, R., Van Der Geest, H. G., Admiraal, W., Cleutjens, J. P. M., Schutte, B., Verheyen, F., Ribes, M., van Loon, E., and de Goeij, J. M. (2014). Cell turnover and detritus production in marine sponges from tropical and temperate benthic ecosystems. PLoS ONE, 9(10): e109486. https://doi.org/10.1371/journal.pone.0109486 
Alva-Basurto, J. C., and Arias-González, J. E. (2014). Modelling the effects of climate change on a Caribbean coral reef food web. Ecological Modelling, 289, 1-14. https://doi.org/10.1016/j.ecolmodel.2014.06.014

Alvarez-Filip, L., Dulvy, N. K., Gill, J. A., Côté, I. M., and Watkinson, A. R. (2009). Flattening of Caribbean coral reefs: Region-wide declines in architectural complexity. Proceedings of the Royal Society B: Biological Sciences, 276: 3019-3025. https://doi.org/10.1098/rspb.2009.0339

Anderson, M. J. (2001). A new method for non-parametric multivariate analysis of variance. Austral Ecology, 26: 32-46.

Anderson, M. J., and Willis T. J. (2003). Canonical Analysis of Principal Coordinates: a useful method of constrained ordination for ecology. Ecology, 84: 511-525.

Anthony, K. R. N., Kline, D. I., Diaz-Pulido, G., Dove, S., and Hoegh-Guldberg, O. (2008). Ocean acidification causes bleaching and productivity loss in coral reef builders. Proceedings of the National Academy of Sciences, 105(45):17442-17446. https://doi.org/10.1073/pnas.0804478105

Archer, S. K., Stevens, J. L., Rossi, R. E., Matterson, K. O., and Layman, C. A. (2017). Abiotic conditions drive significant variability in nutrient processing by a common Caribbean sponge, Ircinia felix. Limnology and Oceanography, 62:1783-1793. https://doi.org/10.1002/lno.10533

Arnold, S. N., and Steneck, R. S. (2011). Settling into an increasingly hostile world: The rapidly closing "recruitment window" for corals. PLoS ONE, 6(12):e28681. https://doi.org/10.1371/journal.pone.0028681

Audzijonyte, A., Pethybridge, H., Porobic, J., Gorton, R., Kaplan, I., and Fulton, E. (2019). Atlantis: a spatially explicit end-to-end marine ecosystem model with dynamically integrated physics, ecology and socio-economics modules. Methods in Ecology and Evolution, 00:1-6. https://doi.org/10.1111/2041-210X.13272

Austin M.P. (1981) Permanent quadrats: An interface for theory and practice. In: Poissonet P., Romane F., Austin M.A., van der Maarel E., Schmidt W. (eds) Vegetation dynamics in grasslands, heathlands and mediterranean ligneous formations. Advances in vegetation science, vol 4. Springer, Dordrecht. 
Babcock, R. C., Dambacher, J. M., Morello, E. B., Plagányi, É. E., Hayes, K. R., Sweatman, H. P. A., and Pratchett, M. S. (2016). Assessing different causes of Crown-of-Thorns starfish outbreaks and appropriate responses for management on the Great Barrier Reef. PLoS ONE, 11(12):e0169048. https://doi.org/10.1371/journal.pone.0169048

Bailey-Brock, J. H. (1987). Fouling community development on an artificial reef in Hawaiian waters. Bulletin of Marine Science, 44(2):580-591.

Baird, M. E., Adams, M. P., Babcock, R. C., Oubelkheir, K., Mongin, M., Wild-Allen, K. A., Skerratt, J., Robson, B. J., Petrou, K., Ralph, P. J., O’Brien, K. R., Carter, A. B., Jarvis, J. C., and Rasheed, M. A. (2016). A biophysical representation of seagrass growth for application in a complex shallow-water biogeochemical model. Ecological Modelling, 325:13-27. https://doi.org/10.1016/j.ecolmodel.2015.12.011

Baird, A. H., Pratchett, M. S., Hoey, A. S., Herdiana, Y. and Campbell, S. J. (2013). Acanthaster planci is a major cause of coral mortality in Indonesia. Coral Reefs, 32(3):803812.

Bannister, R. J., Battershill, C. N., and de Nys, R. (2010). Demographic variability and longterm change in a coral reef sponge along a cross-shelf gradient of the Great Barrier Reef. Marine and Freshwater Research, 61(4):389-396. https://doi.org/10.1071/MF09067

Barnes, D. K. A., and Bell, J. J. (2002). Coastal sponge communities of the West Indian Ocean: morphological richness and diversity. African Journal of Ecology, 40:350-359.

Beaulieu, S. E. (2001). Life on glass houses: Sponge stalk communities in the deep sea. Marine Biology, 138:803-817. https://doi.org/10.1007/s002270000500

Beazley, L. I., Kenchington, E. L., Murillo, F. J., and Sacau, M. del M. (2013). Deep-sea sponge grounds enhance diversity and abundance of epibenthic megafauna in the Northwest Atlantic. ICES Journal of Marine Science, 70(7):1471-1490. https://doi.org/10.1093/icesjms/fst124

Bell, J. J. (2008). The functional roles of marine sponges. Estuarine, Coastal and Shelf Science, 79(3):341-353. https://doi.org/10.1016/j.ecss.2008.05.002

Bell, J. J. (2007). Contrasting patterns of species and functional composition of coral reef sponge assemblages. Marine Ecology Progress Series, 339:73-81. https://doi.org/10.3354/meps339073 
Bell, J. J., and Barnes, D. K. A. (2003). The importance of competitor identity, morphology and ranking methodology to outcomes in interference competition between sponges. Marine Biology, 143:415-426.

Bell, J. J., and Barnes, D. K. A. (2000a). The distribution and prevalence of sponges in relation to environmental gradients within a temperate sea lough: vertical cliff surfaces. Diversity and Distribution, 6:283-303.

Bell, J. J., and Barnes, D. K. A. (2000b). The distribution and prevalence of sponges in relation to environmental gradients within a temperate sea lough: inclined cliff surfaces. Diversity and Distribution, 6:305-323.

Bell, J. J., and Barnes, D. K. A. (2000c). The influences of bathymetry and flow regime upon the morphology of sublittoral sponge communities. Journal of the Marine Biological Association of the United $\quad$ Kingdom, https://doi.org/10.1017/S0025315400002538

Bell, J. J., and Smith, D. (2004). Ecology of sponge assemblages (Porifera) in the Wakatobi region, south-east Sulawesi, Indonesia: richness and abundance. Journal of the Marine Biological Association of the United Kingdom, 84(3):581-591.

Bell, J. J., Rovellini, A., Davy, S. K., Taylor, M. W., Fulton, E. A., Dunn, M. R., Bennett, H. M., Kandler, N. M., Luter, H. M., and Webster, N. S. (2018a). Climate change alterations to ecosystem dominance: how might sponge-dominated reefs function? Ecology, 99(9): 19201931. https://doi.org/10.1002/ecy.2446

Bell, J. J., Bennett, H. M., Rovellini, A., and Webster, N. S. (2018b). Sponges to be winners under near-future climate scenarios. Bioscience 68(12):955-968. https://doi.org/10.1093/biosci/biy142

Bell, J. J., Jompa, J., Haris, A., Werorilangi, S., Shaffer, M., and Mortimer, C. (2018c). Domination of mesophotic ecosystems in the Wakatobi Marine National Park (Indonesia) by sponges, soft corals and other non-hard coral species. Journal of the Marine Biological Association of the United Kingdom, 1-5.

Bell, J. J., Biggerstaff, A., Bates, T., Bennett, H., Marlow, J., McGrath, E. and Shaffer, M. (2017). Sponge monitoring: Moving beyond diversity and abundance measures. Ecological Indicators, 78:470-488. 
Bell, J. J., McGrath, E., Biggerstaff, A., Bates, T., Cárdenas, C. A., and Bennett, H. (2015a). Global conservation status of sponges. Conservation Biology, 29(1):42-53. https://doi.org/10.1111/cobi.12447

Bell, J. J., McGrath, E., Biggerstaff, A., Bates, T., Bennett, H., Marlow, J., and Shaffer, M. (2015b). Sediment impacts on marine sponges. Marine Pollution Bulletin, 94:5-13. https://doi.org/10.1016/j.marpolbul.2015.03.030

Bell, J. J., Smith, D., Hannan, D., Haris, A., Jompa, J., and Thomas, L. (2014). Resilience to disturbance despite limited dispersal and self-recruitment in tropical barrel sponges: implications for conservation and management. PLoS ONE, 9(3):e91635.

Bell, J. J., Davy, S. K., Jones, T., Taylor, M. W., and Webster, N. S. (2013). Could some coral reefs become sponge reefs as our climate changes? Global Change Biology, 19(9):26132624. https://doi.org/10.1111/gcb.12212

Bellwood, D. R., Streit, R. P., Brandl, S. J., and Tebbett, S. B. (2019). The meaning of the term 'function' in ecology: A coral reef perspective. Functional Ecology, 33:948-961. https://doi.org/10.1111/1365-2435.13265

Bender, E. A., Case, T. J., and Gilpin, M. E. (1984). Perturbation experiments in community ecology: theory and practice. Ecology, 65(1):1-13.

Bennett, H. M., Bell, J. J., Davy, S. K., Webster, N. S., and Francis, D. S. (2018). Elucidating the sponge stress response; lipids and fatty acids can facilitate survival under future climate scenarios. Global Change Biology, 24:3130-3144. https://doi.org/10.1111/gcb.14116

Bennett, H. M., Altenrath, C., Woods, L., Davy, S. K., Webster, N. S., and Bell, J. J. (2017). Interactive effects of temperature and $p \mathrm{CO}_{2}$ on sponges: from the cradle to the grave. Global Change Biology, 23:2031-2046. https://doi.org/10.1111/gcb.13474

Berman, J., and Bell, J. J. (2016). Short-term temporal variability in a temperate sponge assemblage. Marine Biology, 163:1-9.

Bertolino, M., Oprandi, A., Santini, C., Castellano, M., Pansini, M., Boyer, M., and Bavestrello, G. (2017). Hydrothermal waters enriched in silica promote the development of a sponge community in North Sulawesi (Indonesia). The European Zoological Journal, 84:128135. 
Bessell-Browne, P., Negri, A. P., Fisher, R., Clode, P. L., Duckworth, A., and Jones, R. (2017). Impacts of turbidity on corals: The relative importance of light limitation and suspended sediments. Marine Pollution Bulletin, 117: 161-170. https://doi.org/10.1016/j.marpolbul.2017.01.050

Biggerstaff, A., Smith, D. J., Jompa, J., and Bell, J. J. (2017a). Metabolic responses of a phototrophic sponge to sedimentation supports transitions to sponge-dominated reefs. Scientific Reports, 7:2725. https://doi.org/10.1038/s41598-017-03018-y

Biggerstaff, A., Jompa, J., and Bell, J. J. (2017b). Increasing benthic dominance of the phototrophic sponge Lamellodysidea herbacea on a sedimented reef within the Coral Triangle. Marine Biology, 164(12): 1-16. https://doi.org/10.1007/s00227-017-3253-3

Biggerstaff, A., Smith, D. J., Jompa, J., and Bell, J. J. (2015). Photoacclimation supports environmental tolerance of a sponge to turbid low-light conditions. Coral Reefs, 34(4): 10491061. https://doi.org/10.1007/s00338-015-1340-9

Birkeland, C. (1977). The importance of rate of biomass accumulation in early succession stages of benthic communities to the survival of coral recruits. In: Proceedings of the third international coral reef symposium, pp 16-21.

Blackwood, J. C., Hastings, A., and Mumby, P. J. (2011). A model-based approach to determine the long-term effects of multiple interacting stressors on coral reefs. Ecological Applications, 21(7): 2722-2733. https://doi.org/10.1890/10-2195.1

Blanquer, A., Uriz, M. J., and Caujapé-Castells, J. (2009). Small-scale spatial genetic structure In Scopalina lophyropoda, an encrusting sponge with philopatric larval dispersal and frequent fission and fusion events. Marine Ecology Progress Series, 380:95-102.

Bock, C. E., Jones, Z. F., and Bock, J. H. (2007). Relationships between species richness, evenness, and abundance in a southwestern savanna. Ecology, 88(5):1322-1327.

Boschetti, S. T. (2016). Impact of the local environmental variability on the patterns of coral recruitment on Indo-Pacific reefs. PhD Thesis, Victoria University of Wellington New Zealand, $157 \mathrm{pp}$.

Boury-Esnault, N., and Rützler, K. (1997). Thesaurus of sponge morphology. Smithsonian Contributions to Zoology, 596:1-55. https://doi.org/10.5479/si.00810282.596 
Bozec, Y. M., Alvarez-Filip, L., and Mumby, P. J. (2015). The dynamics of architectural complexity on coral reefs under climate change. Global Change Biology, 21:223-235. https://doi.org/10.1111/gcb.12698

Bradbury, R. H., and Reichelt, R. E. (1983). Fractal Dimension of a Coral Reef at Ecological Scales. Marine Ecology Progress Series, 10:169-171.

Brandl, S. J., Goatley, C. H. R., Bellwood, D. R., and Tornabene, L. (2018). The hidden half: ecology and evolution of cryptobenthic fishes on coral reefs. Biological Reviews, 93:18461873. https://doi.org/10.1111/brv.12423

Brandl, S. J., Tornabene, L., Goatley, C. H. R., Casey, J. M., Morais, R. A., Côté, I. M., Baldwin, C. C., Parravicini, V., Schiettekatte, N. M. D., and Bellwood, D. R. (2019). Demographic dynamics of the smallest marine vertebrates fuel coral-reef ecosystem functioning. Science, 364(6446):1189-1192. https://doi.org/10.1038/45533

Brey, T., Müller-Wiegmann, C., Zittier, Z. M. C., and Hagen, W. (2010). Body composition in aquatic organisms - A global data bank of relationships between mass, elemental composition and energy content. Journal of Sea Research, 64:334-340. https://doi.org/10.1016/j.seares.2010.05.002

Brown, C. J., and Hamilton, R. J. (2018). Estimating the footprint of pollution on coral reefs with models of species turnover. Conservation Biology, 32(4):949-958. https://doi.org/10.1111/cobi.13079

Bruggemann, J. H., Kessel, A. M. Van, van Rooij, J. M., and Breeman, A. M. (1996). Bioerosion and sediment ingestion by the Caribbean parrotfish Scarus vetula and Sparisoma viride: implications of fish size, feeding mode and habitat use. Marine Ecology Progress Series, 134:59-71.

Bruno, J. F., and Selig, E. R. (2007). Regional decline of coral cover in the Indo-Pacific: Timing, extent, and subregional comparisons. PLoS ONE 2(8):e711. doi:10.1371/journal.pone.0000711

Bruno, J. F., Sweatman, H., Precht, W. F., Selig, E. R., and Schutte, V. G. W. (2009). Assessing evidence of phase shifts to macroalgal dominance on coral reefs. Ecology, 90(6):1478-1484. https://doi.org/10.1890/08-1781.1 
Buhl-Mortensen, L., Vanreusel, A., Gooday, A. J., Levin, L. A., Priede, I. G., BuhlMortensen, P., Gheerardyn, H., King, N., and Raes, M. (2010). Biological structures as a source of habitat heterogeneity and biodiversity on the deep ocean margins. Marine Ecology, 31:2150. https://doi.org/10.1111/j.1439-0485.2010.00359.x

Bulman, C. (2006). North West Shelf Joint Environmental Management Study. Trophic webs and modelling of Australia's North West Shelf. Technical Report no. 9.

Burlando, B., Bavestrello, G., and Arillo, A. (1992). Seasonal changes in the metabolism of the calcareous sponge Clathrina clathrus (Schmidt). Comparative Biochemistry and Phisiology - A: Physiology, 101(2):341-344.

Burns, J., Delparte, D., Gates, R., and Takabayashi, M. (2015). Integrating structure-frommotion photogrammetry with geospatial software as a novel technique for quantifying 3D ecological characteristics of coral reefs. PeerJ, 3:e1077. https://doi.org/10.7717/peerj.1077

Buss, L. W. (1976). Better living through chemistry: the relationship between allelochemical interactions and competitive networks. In: F. W. Harrison (Ed), Aspects of sponge biology, Academic Press, New York, pp 315-327.

Butler, M. J., Hunt, J. H., Herrnkind, W. F., Childress, M. J., Bertelsen, R., Sharp, W., Matthews, T., Field, J. M., and Marshall, H. J. (1995). Cascading disturbances in Florida Bay, USA: cyanobacteria blooms, sponge mortality, and implications for juvenile spiny lobsters Panulirus argus. Marine Ecology Progress Series, 129:119-125.

Calcinai, B., Bastari, A., Bavestrello, G., Bertolino, M., Horcajadas, S. B., Pansini, M., Makapedua, D. M., and Cerrano, C. (2017). Demosponge diversity from North Sulawesi, with the description of six new species. Zookeys 680:105-150.

Calcinai, B., Bertolino, M., Pica, D., Cerrano, C., Bavestrello, G., and Wagner, D. (2013). Sponges associated with octocorals in the Indo-Pacific, with the description of four new species. Zootaxa 3617(1):001-061.

Calcinai, B., Cerrano, C., Totti, C., Romagnoli, T., and Bavestrello, G. (2006). Symbiosis of Mycale (Mycale) vansoesti sp. nov. (Porifera, Demospongiae) with a coralline alga from North Sulawesi (Indonesia). Invertebrate Biology, 125(3):195-204.

Carballo, J. L. (2006). Effect of natural sedimentation on the structure of tropical rocky sponge assemblages. Ecoscience 13(1):119-130. 
Carballo, J. L., Vega, C., Cruz-Barraza, J. A., Yáñez, B., Nava, H., Ávila, E., and Wilson, M. (2008). Short- and long-term patterns of sponge diversity on a rocky tropical coast: Evidence of large-scale structuring factors. Marine Ecology, 29:216-236.

Cardone, F., Nonnis Marzano, C., Spedicato, M. T., Lembo, G., Gaino, E., and Corriero, G. (2008). Budding induction in a marine sponge: a cue for cultivation purposes. IFOAM Conference on Organic Aquaculture, Cattolica - Italy, 18 - 20 June 2008.

Chaves-Fonnegra, A., Zea, S., and Gómez, M. L. (2007). Abundance of the excavating sponge Cliona delitrix in relation to sewage discharge at San Andrés Island, Sw Caribbean, Colombia. Bulletin of Marine and Coastal Research, 36:63-78. https://doi.org/10.25268/bimc.invemar.2007.36.0.201

Chaves-Fonnegra, A., Riegl, B., Zea, S., Lopez, J. V., Smith, T., Brandt, M., and Gilliam, D. S. (2017). Bleaching events regulate shifts from corals to excavating sponges in algaedominated reefs. Global Change Biology, 24:773-785. https://doi.org/10.1111/gcb.13962

Cheal, A. J., MacNeil, M. A., Emslie, M. J., and Sweatman, H. (2017). The threat to coral reefs from more intense cyclones under climate change. Global Change Biology, 23:15111524. https://doi.org/10.1111/gcb.13593

Cheshire, A. C., and Wilkinson, C. R. (1991). Modelling the photosynthetic production by sponges on Davies Reef, Great Barrier Reef. Marine Biology, 109:13-18.

Cheshire, A. C., Wilkinson, C. R., Seddon, S., and Westphalen, G. (1997). Bathymetric and seasonal changes in photosynthesis and respiration of the phototrophic sponge Phyllospongia lamellosa in comparison with respiration by the heterotrophic sponge Ianthella basta on Davies Reef, Great Barrier Reef. Marine and Freshwater Research, 48(7):589-599. https://doi.org/10.1071/MF96070

Cheshire, A., Butler, A., Westphalen, G., Rowland, B., Stevenson, J., and Wilkinson, C. (1995). Preliminary study of the distribution and photophysiology of the temperate phototrophic sponge Cymbastela sp. from South Australia. Marine and Freshwater Research, 46: 1211-1216. https://doi.org/10.1071/MF9951211

Clarke, K. R. (1993). Non-parametric multivariate analyses of changes in community structure. Australian Journal of Ecology, 18(1): 117-143. https://doi.org/10.1111/j.14429993.1993.tb00438.x 
Cleary, D. F. R., and de Voogd, N. J. (2007). Environmental associations of sponges in the Spermonde Archipelago, Indonesia. Journal of the Marine Biological Association of the United Kingdom, 87:1669-1676.

Cleary, D. F. R., Swierts, T., Coelho, F. J. R. C., Polónia, A. R. M., Huang, Y. M., Ferreira, M. R. S., Putchakarn, S., Carvalheiro, L., van der Ent, E., Ueng, J. P., Gomes, N. C. M., and de Voogd, N. J. (2019). The sponge microbiome within the greater coral reef microbial metacommunity. Nature Communications, 10(1): 1-12. https://doi.org/10.1038/s41467-01909537-8

Clifton, J., and Unsworth, R. K. (2010). Introduction. In Clifton J., Unsworth, R. K., and Smith, D. J. (Eds.), Marine research and conservation in the Coral Triangle: the Wakatobi National Park (pp. 11-26). New York: Nova Publishers.

Clifton, J., Unsworth, R. K., and Smith, D. J. (2010). Marine research and conservation in the Coral Triangle: the Wakatobi National Park. Environmental Science, Engineering and Technology Series. Nova Publishers, New York, 274 pp.

Coker, D. J., Wilson, S. K., and Pratchett, M. S. (2014). Importance of live coral habitat for reef fishes. Reviews in Fish Biology and Fisheries, 24:89-126. https://doi.org/10.1007/s11160013-9319-5

Coles, S. L., and Bolick, H. (2007). Invasive introduced sponge Mycale grandis overgrows reef corals in Kāne`ohe Bay, O`ahu, Hawai'i. Coral Reefs, 26:911. https://doi.org/10.1007/s00338-007-0295-x

Collie, J. S., Botsford, L. W., Hastings, A., Kaplan, I. C., Largier, J. L., Livingston, P. A., Plagányi, É., Rose, K. A., Wells, B. K., and Werner, F. E. (2014). Ecosystem models for fisheries management: finding the sweet spot. Fish and Fisheries, 17:101-125. https://doi.org/10.1111/faf.12093

Colvard, N. B., and Edmunds, P. J. (2011). Decadal-scale changes in abundance of nonscleractinian invertebrates on a Caribbean coral reef. Journal of Experimental Marine Biology and Ecology, 397(2), 153-160. https://doi.org/10.1016/j.jembe.2010.11.015

Connell, J. H., Hughes, T. P., and Wallace, C. C. (1997). A 30-year study of coral abundance, recruitment, and disturbance at several scales in space and time. Ecological Monographs, 67(4):461-488. 
Connell, S. D., and Russell, B. D. (2010). The direct effects of increasing $\mathrm{CO}_{2}$ and temperature on non-calcifying organisms: Increasing the potential for phase shifts in kelp forests. Proceedings of the Royal Society B: Biological Sciences, 277:1409-1415. https://doi.org/10.1098/rspb.2009.2069

Conway, K. W., Krautter, M., Barrie, J. V., and Neuweiler, M. (2001). Hexactinellid sponge reefs on the Canadian continental shelf: A unique "living fossil." Geoscience Canada, 28(2), $71-78$.

Cooper, J. K., Spencer, M., and Bruno, J. F. (2015). Stochastic dynamics of a warmer Great Barrier Reef. Ecology 96(7):1802-1811.

Cornwall, C. E., Revill, A. T., Hall-Spencer, J. M., Milazzo, M., Raven, J. A., and Hurd, C. L. (2017). Inorganic carbon physiology underpins macroalgal responses to elevated $\mathrm{CO}_{2}$. Scientific Reports, 7: 46297. https://doi.org/10.1038/srep46297

Crabbe, M. J. C., and Smith, D. J. (2005). Sediment impacts on growth rates of Acropora and Porites corals from fringing reefs of Sulawesi, Indonesia. Coral Reefs, 24:437-441.

Cribari-Neto, F., and Zeileis, A. (2010). Beta Regression in R. Journal of Statistical Software, 1(2). https://doi.org/10.18637/jss.v034.i02

Crisp, D. J., and Ryland, J. S. (1960). Influence of filming and of surface texture on the settlement of marine organisms. Nature, 185(4706):119.

Crossland, C. J., Hatcher, B. G., and Smith, S. V. (1991). Role of coral reefs in global ocean production. Coral Reefs, 10:55-64. https://doi.org/10.1007/BF00571824

Crutzen, P. J. (2006). The “Anthropocene”. In Ehlers, E., and Krafft, T. (Eds.), Earth system science in the Anthropocene (pp. 13-18). Springer, Berlin, Heidelberg.

Cruz, I. C. S., de Kikuchi, R. K. P., Longo, L. L., and Creed, J. C. (2015). Evidence of a phase shift to Epizoanthus gabrieli Carlgreen, 1951 (Order Zoanthidea) and loss of coral cover on reefs in the Southwest Atlantic. Marine Ecology, 36:318-325.

Curtis-Quick, J. (2013). Drivers of change of reef fish assemblages within the Coral Triangle. PhD Thesis, University of Essex, 294 pp. 
Dambacher, J. M., Rothlisberg, P. C., and Loneragan, N. R. (2015). Qualitative mathematical models to support ecosystem-based management of Australia's Northern Prawn Fishery. Ecological Applications, 25(1): 278-298. https://doi.org/10.1890/13-2030.1

Dambacher, J. M., Hodge, K. B., Babcock, R. C., Fulton, E. A., Apte, S. C., Plagányi, É. E., Warne, M. St. J., and Marshall, N. A. (2013). Models and Indicators of Key Ecological Assets in Gladstone Harbour. A report prepared for the Gladstone Healthy Harbour Partnership. CSIRO Wealth from Oceans Flagship, Hobart.

Dambacher, J. M., Gaughan, D. J., Rochet, M. J., Rossignol, P. A., and Trenkel, V. M. (2009). Qualitative modelling and indicators of exploited ecosystems. Fish and Fisheries, 10(3): 305322. https://doi.org/10.1111/j.1467-2979.2008.00323.x

Dambacher, J. M., Brewer, D. T., Dennis, D. M., Macintyre, M., and Foale, S. (2007). Qualitative modelling of gold mine impacts on Lihir Island's socioeconomic system and reefedge fish community. Environmental Science and Technology, 41(2):555-562. https://doi.org/10.1021/es0610333

Dambacher, J. M., Li, H. W., and Rossignol, P. A. (2003a). Qualitative predictions in model ecosystems. Ecological Modelling, 161:79-93. https://doi.org/10.1016/S0304-3800(02)002958

Dambacher, J. M., Luh, H.-K., Li, H. W., and Rossignol, P. A. (2003b). Qualitative stability and ambiguity in model ecosystems. The American Naturalist, 161(6):876-888. https://doi.org/10.1086/367590

Dambacher, J. M., Li, H. W., and Rossignol, P. A. (2002). Relevance of community structure in assessing indeterminacy of ecological predictions. Ecology, 83(5):1372-1385.

Darling, E. S., McClanahan, T. R., Maina, J., Gurney, G. G., Graham, N. A. J., JanuchowskiHartley, F., Cinner, J. E., Mora, C., Hicks, C. C., Maire, E., Puotinen, M., Skirving, W. J., Adjeroud, M., Ahmadia, G., Arthur, R., Bauman, A. G., Beger, M., Berumen, M. L., Bigot L., Bouwmeester, J., Brenier, A., Bridge, T. C. L., Brown, E., Campbell, S. J., Cannon, S., Cauvin, B., Chen, C. A., Claudet, J., Denis, V., Donner, S., Estradivari, Fadli, N., Feary, D. A., Fenner, D., Fox, H., Franklin, E. C., Friedlander, A., Gilmour, J., Goiran, C., Guest, J., Hobbs, J. A., Hoey, A. S., Houk, P., Johnson, S., Jupiter, S. D., Kayal, M., Kuo, C. Y., Lamb, J., Lee, M. A. C., Low, J., Muthiga, N., Muttaqin, E., Nand, Y., Nash, K. L., Nedlic, O., Pandolfi, J. M., Pardede, S., Patankar, V., Penin, L., Ribas-Deulofeu, L., Richards, Z., Roberts, T. E., Rodgers, 
K. S., Safuan, C. D. M., Sala, E., Shedrawi, G., Sin, T. M., Smallhorn-West, P., Smith, J. E., Sommer, B., Steinberg, P. D., Sutthacheep, M., Tan, C. H. J., Williams, G. J., Wilson, S., Yeemin, T., Bruno, J. F., Fortin, M. J., Krkosek, M., and Mouillot, D. (2019). Socialenvironmental drivers inform strategic management of coral reefs in the Anthropocene. Nature Ecology and Evolution. https://doi.org/10.1038/s41559-019-0953-8

Darling, E. S., Graham, N. A. J., Januchowski-Hartley, F. A., Nash, K. L., Pratchett, M. S., and Wilson, S. K. (2017). Relationships between structural complexity, coral traits, and reef fish assemblages. Coral Reefs, 36:561-575. https://doi.org/10.1007/s00338-017-1539-z

Davies, G. M., and Gray, A. (2015). Don't let spurious accusations of pseudoreplication limit our ability to learn from natural experiments (and other messy kinds of ecological monitoring). Ecology and Evolution, 5(22):5295-5304.

de Goeij, J. M., and van Duyl, F. C. (2007). Coral cavities are sinks of dissolved organic carbon (DOC). Limnology and Oceanography, 52(6):2608-2617. https://doi.org/10.4319/1o.2007.52.6.2608

de Goeij, J. M., van Oevelen, D., Vermeij, M. J. A., Osinga, R., Middelburg, J. J., de Goeij, A. F. P. M., and Admiraal, W. (2013). Surviving in a marine desert: the sponge loop retains resources within coral reefs. Science, 342(6154):108-110. https://doi.org/10.1126/science.1241981

de Goeij, J. M., De Kluijver, A., Van Duyl, F. C., Vacelet, J., Wijffels, R. H., De Goeij, A. F. P. M., Cleutjens, J. P. M., and Schutte, B. (2009). Cell kinetics of the marine sponge Halisarca caerulea reveal rapid cell turnover and shedding. Journal of Experimental Biology, 212(23), 3892-3900. https://doi.org/10.1242/jeb.034561

de Goeij, Jasper M., Van Den Berg, H., Van Oostveen, M. M., Epping, E. H. G., and Van Duyl, F. C. (2008). Major bulk dissolved organic carbon (DOC) removal by encrusting coral reef cavity sponges. Marine Ecology Progress Series, 357:139-151. https://doi.org/10.3354/meps07403

de Ruyter van Steveninck, E., and Breeman, A. (1981). Biomass and relative coverage of benthic algae in the fore-reef of Curaçao (Netherlands Antilles) in relation to production. Marine Ecology Progress Series, 6:257-265. https://doi.org/10.3354/meps006257 
de Voogd, N. J., and Cleary, D. F. R. (2008). An analysis of sponge diversity and distribution at three taxonomic levels in the Thousand Islands/Jakarta Bay reef complex, West-Java, Indonesia. Marine Ecology, 29:205-215.

de Voogd, N. J., Becking, L. E., and Cleary, D. F. R. (2009). Sponge community composition in the Derawan Islands, NE Kalimantan, Indonesia. Marine Ecology Progress Series, 396:169180.

de Voogd, N. J., Becking, L. E., Hoeksema, B. W., Noor, A., and Van Soest, R. W. M. (2004). Sponge interactions with spatial competitors in the Spermonde Archipelago. Bolletino Di Museo e Istituto Di Biologia Dell'Universita Di Genova, 68:253-261. Retrieved from http://www.narcis.nl/publication/RecordID/oai:uva.nl:158420

de Vos, L., Rützler, K., Boury-Esnault, N., Donadey, C., and Vacelet, J. (1991). Atlas of sponge morphology. Washington: Smithsonian Institution Press.

Denis, V., Ribas-Deulofeu, L., Sturaro, N., Kuo, C.-Y., and Chen, C. A. (2017). A functional approach to the structural complexity of coral assemblages based on colony morphological features. Scientific Reports, 7:9849. https://doi.org/10.1038/s41598-017-10334-w

Diaz, M. C., and Rutzler, K. (2001). Sponges: an essential component of Caribbean coral reefs. Bulletin of Marine Science, 69(2):535-546.

Diaz, M. C., and Ward, B. B. (1997). Sponge-mediated nitrification in tropical benthic communities. Marine Ecology Progress Series, 156:97-107. https://doi.org/10.3354/meps156097

Dinno, A. (2017). dunn.test: Dunn's Test of Multiple Comparisons Using Rank Sums. R package version 1.3.5. Available at: https://cran.r-project.org/package=dunn.test

Duckworth, A. R., and Peterson, B. J. (2012). Effects of seawater temperature and pH on the boring rates of the sponge Cliona celata in scallop shells. Marine Biology, 160(1):27-35. https://doi.org/10.1007/s00227-012-2053-z

Duckworth, A. R., West, L., Vansach, T., Stubler, A., and Hardt, M. (2012). Effects of water temperature and $\mathrm{pH}$ on growth and metabolite biosynthesis of coral reef sponges. Marine Ecology Progress Series, 462:67-77. https://doi.org/10.3354/meps09853 
Dudgeon, S. R., Aronson, R. B., Bruno, J. F., and Precht, W. F. (2010). Phase shifts and stable states on coral reefs. Marine Ecology Progress Series, 413:201-216. https://doi.org/10.3354/meps08751

Dullo, W. C. (2005). Coral growth and reef growth: A brief review. Facies, 51(1-4): 33-48. https://doi.org/10.1007/s10347-005-0060-y

Dunlap, M., and Pawlik, J. R. (1996). Video-monitored predation by Caribbean reef fishes on an array of mangrove and reef sponges. Marine Biology, 126:117-123. https://doi.org/10.1007/BF00571383

Easson, C. G., Slattery, M., Baker, D. M., and Gochfeld, D. J. (2014). Complex ecological associations: Competition and facilitation in a sponge-algal interaction. Marine Ecology Progress Series, 507:153-167. https://doi.org/10.3354/meps 10852

Edinger, E. N., Jompa, J., Limmon, G. V., Widjatmoko, W., and Risk, M. J. (1998). Reef degradation and coral biodiversity in Indonesia: Effects of land-based pollution, destructive fishing practices and changes over time. Marine Pollution Bulletin, 36(8):617-630.

Edmunds, P. J., Burgess, S. C., Putnam, H. M., Baskett, M. L., Bramanti, L., Fabina, N. S., Han, X., Lesser, M. P., Madin, J. S., Wall, C. B., Yost, D. M., and Gates, R. D. (2014). Evaluating the causal basis of ecological success within the Scleractinia: an integral projection model approach. Marine Biology, 161:2719-2734. https://doi.org/10.1007/s00227-014-2547$\mathrm{y}$

Elliott, J. A., Patterson, M. R., Staub, C. G., Koonjul, M., and Elliott, S. M. (2018). Decline in coral cover and flattening of the reefs around Mauritius (1998-2010). PeerJ, 6:e6014. https://doi.org/10.7717/peerj.6014

Elliott, J. A., Patterson, M. R., Summers, N., Miternique, C., Montocchio, E., and Vitry, E. (2016). How does the proliferation of the coral-killing sponge Terpios hoshinota affect benthic community structure on coral reefs? Coral Reefs, 35(3):1083-1095. https://doi.org/10.1007/s00338-016-1434-z

Emanuel, K. A. (2013). Downscaling CMIP5 climate models shows increased tropical cyclone activity over the 21st century. Proceedings of the National Academy of Sciences, 110(30): 12219-12224. https://doi.org/10.1073/pnas.1301293110 
Emslie, M. J., Cheal, A. J., and Johns, K. A. (2014). Retention of habitat complexity minimises disassembly of reef fish communities following disturbance: A large-scale natural experiment. PLoS ONE, 9(8):e105384. https://doi.org/10.1371/journal.pone.0105384

Fang, J. K. H., Mason, R. A. B., Schönberg, C. H. L., Hoegh-Guldberg, O., and Dove, S. (2017). Studying interactions between excavating sponges and massive corals by the use of hybrid cores. Marine Ecology, 38(1): 1-8. https://doi.org/10.1111/maec.12393

Fang, J. K. H., Schönberg, C. H. L., Mello-Athayde, M. A., Hoegh-Guldberg, O., and Dove, S. (2014). Effects of ocean warming and acidification on the energy budget of an excavating sponge. Global Change Biology, 20:1043-1054. https://doi.org/10.1111/gcb.12369

Fang, J. K. H., Mello-Athayde, M. A., Schönberg, C. H. L., Kline, D. I., Hoegh-Guldberg, O., and Dove, S. (2013). Sponge biomass and bioerosion rates increase under ocean warming and acidification. Global Change Biology, 19:3581-3591. https://doi.org/10.1111/gcb.12334

Farnham, E. S., and Bell, J. J. (2018). Spatial variation in a shallow-water sponge-dominated reef in Timor-Leste (East Timor). Pacific Science. 72(2):233-244.

Fay, G., Link, J. S., and Hare, J. A. (2017). Assessing the effects of ocean acidification in the Northeast US using an end-to-end marine ecosystem model. Ecological Modelling, 347:1-10. https://doi.org/10.1016/j.ecolmodel.2016.12.016

Feely, R. A., Sabine, C. L., Lee, K., Berelson, W., Kleypas, J., Fabry, V. J., and Millero, F. J. (2004). Impact of anthropogenic $\mathrm{CO}_{2}$ on the $\mathrm{CaCO}_{3}$ system in the oceans. Science, 305:362366. https://doi.org/10.1126/science.1097329

Ferguson, J. M., Reichert, B. E., Fletcher, R. J., and Jager, H. I. (2017). Detecting populationenvironmental interactions with mismatched time series data. Ecology 98(11):2813-2822.

Ferrari, R., Bryson, M., Bridge, T., Hustache, J., Williams, S. B., Byrne, M., and Figueira, W. (2016). Quantifying the response of structural complexity and community composition to environmental change in marine communities. Global Change Biology, 22:1965-1975. https://doi.org/10.1111/gcb.13197

Ferrari, S., and Cribari-Neto, F. (2004). Beta Regression for modelling rates and proportions. Journal of Applied Statistics, 31(7):799-815. https://doi.org/10.1080/0266476042000214501 
Ferrario, F., Beck, M. W., Storlazzi, C. D., Micheli, F., Shepard, C. C., and Airoldi, L. (2014). The effectiveness of coral reefs for coastal hazard risk reduction and adaptation. Nature Communications, 5:3794. https://doi.org/10.1038/ncomms4794

Finnigan, J. J. (2000). Turbulence in plant canopies. Annual Review of Fluid Mechanics, 32: $519-571$.

Fiore, C. L., and Cox Jutte, P. (2010). Characterization of macrofaunal assemblages associated with sponges and tunicates collected off the southeastern United States. Invertebrate Biology, 129(2):105-120. https://doi.org/10.1111/j.1744-7410.2010.00184.x

Frid, C. L. J., Garwood, P. R., and Robinson, L. A. (2009). Observing change in a North Sea benthic system: A 33 year time series. Journal of Marine Systems, 77:227-236.

Friedman, A., Pizarro, O., Williams, S. B., and Johnson-Roberson, M. (2012). Multi-scale measures of rugosity, slope and aspect from benthic stereo image reconstructions. PLoS ONE, 7(12):e50440. https://doi.org/10.1371/journal.pone.0050440

Friedman J., Hastie T., and Tibshirani R. (2010). Regularization paths for Generalized Linear Models via Coordinate Descent. Journal of Statistical Software, 33(1):1-22.

Froese, R. and Pauly, D. (Eds) (2019). FishBase. World Wide Web electronic publication. www.fishbase.org (02/2019).

Fromont, J., Althaus, F., McEnnulty, F. R., Williams, A., Salotti, M., Gomez, O., and Gowlett-Holmes, K. (2012). Living on the edge: The sponge fauna of Australia's southwestern and northwestern deep continental margin. Hydrobiologia, 687:127-142. https://doi.org/10.1007/s10750-011-0845-7

Fulton, C. J., Abesamis, R. A., Berkström, C., Depczynski, M., Graham, N. A. J., Holmes, T. H., Noble, M. M., Radford, B. T., Tano, S., Tinkler, P., Wernberg, T., and Wilson, S. K. (2019). Form and function of tropical macroalgal reefs in the Anthropocene. Functional Ecology, 33:989-999. https://doi.org/10.1111/1365-2435.13282

Fulton, E. A., Bulman, C. M., Pethybridge, H., and Goldsworthy, S. D. (2018). Modelling the Great Australian Bight Ecosystem. Deep-Sea Research Part II: Topical Studies in Oceanography, 157-158(November):211-235. https://doi.org/10.1016/j.dsr2.2018.11.002

Fulton, E. A., Link, J. S., Kaplan, I. C., Savina-Rolland, M., Johnson, P., Ainsworth, C., Horne, P., Gorton, R., Gamble, R. J., Smith, A. D. M., and Smith, D. C. (2011). Lessons in 
modelling and management of marine ecosystems: The Atlantis experience. Fish and Fisheries, 12:171-188. https://doi.org/10.1111/j.1467-2979.2011.00412.x

Fulton, E. A., Hatfield, B., Althaus, F., and Sainsbury, K. J. (2006). Benthic habitat dynamics and models on Australia's North West Shelf. North West Shelf Joint Environmental Management Study. Technical Report No. 11. Hobart, Tasmania, Australia.

Gazave, E., Lapebie, P., Ereskovsky, A. V., Vacelet, J., Renard, E., Cárdenas, P., and Borchiellini, C. (2012). No longer Demospongiae: Homoscleromorpha formal nomination as a fourth class of Porifera. Hydrobiologia, 687:3-10. https://doi.org/10.1007/s10750-011-0842-x

Gemballa, S., and Schermutzki, F. (2004). Cytotoxic haplosclerid sponges preferred: A field study on the diet of the dotted sea slug Peltodoris atromaculata (Doridoidea: Nudibranchia). Marine Biology, 144:1213-1222. https://doi.org/10.1007/s00227-003-1279-1

George, A. M., Brodie, J., Daniell, J., Capper, A., and Jonker, M. (2018). Can sponge morphologies act as environmental proxies to biophysical factors in the Great Barrier Reef, Australia? Ecological Indicators, 93:1152-1162. https://doi.org/10.1016/j.ecolind.2018.06.016

Gerovasileiou, V., Chintiroglou, C. C., Konstantinou, D., and Voultsiadou, E. (2016). Sponges as "living hotels" in Mediterranean marine caves. Scientia Marina, 80(3):279-289. https://doi.org/10.3989/scimar.04403.14b

Gilmour, J. P., Smith, L. D., Heyward, A. J., Baird, A. H., and Pratchett, M. S. (2013). Recovery of an isolated coral reef system following severe disturbance. Science, 340:69-71. https://doi.org/10.1126/science.1232310

Gonzalez-Andujar, J. L., Fernandez-Quintanilla, C., and Navarrete, L. (2006). Population cycles produced by delayed density dependence in an annual plant. The American Naturalist, $168(3): 318-322$.

González-Rivero, M., Harborne, A. R., Herrera-Reveles, A., Bozec, Y. M., Rogers, A., Friedman, A., Ganase, A., and Hoegh-Guldberg, O. (2017). Linking fishes to multiple metrics of coral reef structural complexity using three-dimensional technology. Scientific Reports, 7:13965. https://doi.org/10.1038/s41598-017-14272-5

González-Rivero, M., Bozec, Y. M., Chollett, I., Ferrari, R., Schönberg, C. H. L., and Mumby, P. J. (2016). Asymmetric competition prevents the outbreak of an opportunistic 
species after coral reef degradation. Oecologia, 181:161-173. https://doi.org/10.1007/s00442015-3541-x

González-Rivero, M., Ferrari, R., Schönberg, C. H. L., and Mumby, P. J. (2012). Impacts of macroalgal competition and parrotfish predation on the growth of a common bioeroding sponge. Marine Ecology Progress Series, 444:133-142. https://doi.org/10.3354/meps09424

González-Rivero, M., Yakob, L., and Mumby, P. J. (2011). The role of sponge competition on coral reef alternative steady states. Ecological Modelling, 222:1847-1853. https://doi.org/10.1016/j.ecolmodel.2011.03.020

Goreau, T. F., and Hartman, W. D. (1966). Sponge: effect on the form of coral reefs. Science, 151(3708):343-344.

Gouraguine, A., Moranta, J., Ruiz-Frau, A., Hinz, H., Reñones, O., Ferse, S. C. A., Jompa, J., and Smith, D. J. (2019). Citizen science in data and resource-limited areas: A tool to detect long-term ecosystem changes. PLoS ONE, 14(1):e0210007.

Graham, N. A. J., Jennings, S., MacNeil, M. A., Mouillot, D., and Wilson, S. K. (2015). Predicting climate-driven regime shifts versus rebound potential in coral reefs. Nature. Nature, 518(7537):94-97.

Graham, N. A. J., Cinner, J. E., Norström, A. V., and Nyström, M. (2014). Coral reefs as novel ecosystems: embracing new futures. Current Opinion in Environmental Sustainability, 7:9-14. https://doi.org/10.1016/j.cosust.2013.11.023

Graham, N. A. J., and Nash, K. L. (2013). The importance of structural complexity in coral reef ecosystems. Coral Reefs, 32:315-326. https://doi.org/10.1007/s00338-012-0984-y

Graham, N. A. J., Wilson, S. K., Jennings, S., Polunin, N. V. C., Bijoux, J. P., and Robinson, J. (2006). Dynamic fragility of oceanic coral reef ecosystems. Proceedings of the National Academy of Sciences, 103(22):8425-8429. https://doi.org/10.3233/THC-151011

Gratwicke, B., and Speight, M. R. (2005). The relationship between fish species richness, abundance and habitat complexity in a range of shallow tropical marine habitats. Journal of Fish Biology, 66:650-667. https://doi.org/10.1111/j.1095-8649.2005.00629.x

Greenwood, J. E., Truesdale, V. W., and Rendell, A. R. (2001). Biogenic silica dissolution in seawater - In vitro chemical kinetics. Progress in Oceanography, 48:1-23. https://doi.org/10.1016/S0079-6611(00)00046-X 
Gribble, N. A. (2003). GBR-prawn: Modelling ecosystem impacts of changes in fisheries management of the commercial prawn (shrimp) trawl fishery in the far northern Great Barrier Reef. Fisheries Research, 65(1-3):493-506. https://doi.org/10.1016/j.fishres.2003.09.035

Griffith, G. P., Fulton, E. A., and Richardson, A. J. (2011). Effects of fishing and acidification-related benthic mortality on the southeast Australian marine ecosystem. Global Change Biology, 17:3058-3074. https://doi.org/10.1111/j.1365-2486.2011.02453.x

Gross, K., Edmunds, P. J., and Holmes, E. E. (2015). Stability of Caribbean coral communities quantified by long-term monitoring and autoregression models. Ecology 96(7):1812-1822.

Guida, V. G. (1976). Sponge predation in the oyster reef community as demonstrated with Cliona celata Grant. Journal of Experimental Marine Biology and Ecology, 25(2):109-122. https://doi.org/10.1016/0022-0981(76)90012-5

Gutierrez-Heredia, L., Benzoni, F., Murphy, E., and Reynaud, E. G. (2016). End to end digitisation and analysis of three-dimensional coral models, from communities to corallites. PLoS ONE, 11(2):e0149641. https://doi.org/10.1371/journal.pone.0149641

Haas, A. F., Fairoz, M. F. M., Kelly, L. W., Nelson, C. E., Dinsdale, E. A., Edwards, R. A., Giles, S., Hatay, M., Hisakawa, N., Knowles, B., Lim, Y. W., Maughan, H., Pantos, O., Roach, T. N. F., Sanchez, S. E., Silveira, C. B., Sandin, S., Smith, J, E., and Rohwer, F. (2016). Global microbialization of coral reefs. Nature Microbiology, 1(6):16042. https://doi.org/10.1038/nmicrobiol.2016.42

Hadas, E., Shpigel, M., and Ilan, M. (2009). Particulate organic matter as a food source for a coral reef sponge. Journal of Experimental Biology, 212:3643-3650. https://doi.org/10.1242/jeb.027953

Hadi, T. A., Hadiyanto, Budiyanto, A., Wentao, N., and Suharsono (2015). Morphological and species diversity of sponges in coral reef ecosystem in the Lembeh Strait, Bitung. Marine Research in Indonesia, 40(2):65-77. https://doi.org/10.14203/mri.v40i2.45

Hall-Spencer, J. M., Rodolfo-Metalpa, R., Martin, S., Ransome, E., Fine, M., Turner, S. M., Rowley, S. J., Tedesco, D., and Buia, M. C. (2008). Volcanic carbon dioxide vents show ecosystem effects of ocean acidification. Nature, 454(7200):96-99. https://doi.org/10.1038/nature07051 
Hamilton, N. E., Ferry, M. (2018). ggtern: Ternary Diagrams using ggplot2. Journal of Statistical Software, Code Snippets, 87(3), 1-17. doi: 10.18637/jss.v087.c03.

Harborne, A. R., Rogers, A., Bozec, Y.-M., and Mumby, P. J. (2017). Multiple stressors and the functioning of coral reefs. Annual Review of Marine Science, 9:445-468. https://doi.org/10.1146/annurev-marine-010816-060551

Harder, T., Lau, S. C. K., Dobretsov, S., Fang, T. K., and Qian, P. Y. (2003). A distinctive epibiotic bacterial community on the soft coral Dendronephthya sp. and antibacterial activity of coral tissue extracts suggest a chemical mechanism against bacterial epibiosis. FEMS Microbiology Ecology, 43(3):337-347. https://doi.org/10.1016/S0168-6496(02)00434-8

Harris, D. L., Rovere, A., Casella, E., Power, H., Canavesio, R., Collin, A., Pomeroy, A., Webstre, J. M., and Parravicini, V. (2018). Coral reef structural complexity provides important coastal protection from waves under rising sea levels. Science Advances, 4:eaao4350. https://doi.org/10.1126/sciadv.aao4350

Heck, K. L., and Wetstone, G. S. (1977). Habitat complexity and invertebrate species richness and abundance in tropical seagrass meadows. Journal of Biogeography, 4(2):135-142. https://doi.org/10.2307/3038158

Henry, L. A., and Hart, M. (2005). Regeneration from injury and resource allocation in sponges and corals - A review. International Review of Hydrobiology, 90(2):125-158. https://doi.org/10.1002/iroh.200410759

Heron, S. F., Maynard, J. A., Van Hooidonk, R., and Eakin, C. M. (2016). Warming trends and bleaching stress of the world's coral reefs 1985-2012. Scientific Reports, 6:38402.

Herrnkind, W. F., Butler IV, M. J., Hunt, J. H., and Childress, M. (1997). Role of physical refugia: Implications from a mass sponge die-off in a lobster nursery in Florida. Marine and Freshwater Research, 48:759-769. https://doi.org/10.1071/MF97193

Heyward, A. J., and Negri, A. P. (1999). Natural inducers for coral larval metamorphosis. Coral Reefs 18:273-279.

Hill, D., Fasham, M., Tucker, G., Shewry, M., and Shaw, P. (Eds) (2006). Handbook of biodiversity methods: Survey, evaluation and monitoring. Cambridge: Cambridge University Press. 
Hixon, M. A., and Beets, J. P. (1993). Predation, prey refuges, and the structure of coral-reef fish assemblages. Ecological Monographs, 63(1):77-101.

Hoegh-Guldberg, O., Mumby, P. J., Hooten, A. J., Steneck, R. S., Greenfield, P., Gomez, E., Harvell, C. D., Sale, P. F., Edwards, A. J., Caldeira, K., Knowlton, N., Eakin, C. M., InglesiasPriesto, R., Muthiga, N., Bradbury, R. H., Dubi, A., and Hatziolos, M. E. (2007). Coral reefs under rapid climate change and ocean acidification. Science, 318:1737-1742.

Hoeksema, B. W., and de Voogd, N. J. (2012). On the run: Free-living mushroom corals avoiding interaction with sponges. Coral Reefs, 31:455-459.

Hoer, D. R., Gibson, P. J., Tommerdahl, J. P., Lindquist, N. L., and Martens, C. S. (2018). Consumption of dissolved organic carbon by Caribbean reef sponges. Limnology and Oceanography, 63(1):337-351. https://doi.org/10.1002/1no.10634

Hoey, A. S., Brandl, S. J. and Bellwood, D. R. (2013). Diet and cross-shelf distribution of rabbitfishes (f. Siganidae) on the northern Great Barrier Reef: Implications for ecosystem function. Coral Reefs 32:973-984.

Hogg, M. M., Tendal, O. S., Conway, K. W., Pomponi, S. A., van Soest, R. W. M., Gutt, J., Krauter, M., and Roberts, J. M. (2010). Deep-sea sponge grounds: Reservoirs of biodiversity. UNEP-WCMC Biodiversity Series No. 32. UNEP-WCMC, Cambridge, UK.

Holbrook, S. J., Schmitt, R. J., Messmer, V., Brooks, A. J., Srinivasan, M., Munday, P. L., and Jones, G. P. (2015). Reef fishes in biodiversity hotspots are at greatest risk from loss of coral species. PLoS ONE, 10(5):e0124054. https://doi.org/10.1371/journal.pone.0124054

Holmer, M. (1996). Composition and fate of dissolved organic carbon derived from phytoplankton detritus in coastal marine sediments. Marine Ecology Progress Series, 141:217228. https://doi.org/10.3354/meps 141217

Hooper, J. N. A., and van Soest, R. W. M. (Eds) (2002). Systema Porifera. Springer US, New York, $1707 \mathrm{pp}$.

Hooper, J. N. A., Kennedy, J. A., List-Armitage, S. E., Cook, S. D., and Quinn, R. (1999). Biodiversity, species composition and distribution of marine sponges in Northeast Australia. Memoirs of the Queensland Museum, 44:263-274.

Hoppe, W. F. (1988). Growth, regeneration and predation in three species of large coral reef sponges. Marine Ecology Progress Series, 50:117-125. https://doi.org/10.1007/BF00301981 
Huang, Z., Brooke, B., and Li, J. (2011). Performance of predictive models in marine benthic environments based on predictions of sponge distribution on the Australian continental shelf. Ecological Informatics, 6(3-4):205-216. https://doi.org/10.1016/j.ecoinf.2011.01.001

Hughes, T. P. (1994). Catastrophes, phase shifts, and large-scale degradation of a Caribbean coral reef. Science, 265(5178): 1547-1551. https://doi.org/10.1126/science.265.5178.1547

Hughes, T. P., Kerry, J. T., and Simpson, T. (2018). Large-scale bleaching of corals on the Great Barrier Reef. Ecology, 99(2):501.

Hughes, T. P., Kerry, J. T., Álvarez-Noriega, M., Álvarez-Romero, J. G., Anderson, K. D., Baird, A. H., Babcock, R. C., Beger, M., Bellwood, D. R., Berkelmans, R., Bridge, T. C., Butler, I. R., Byrne, M., Cantin, N. E., Comeau, S., Connolly, S. R., Cumming, G. S., Dalton, S. J., Diaz-Pulido, G., Eakin, C. M., Figueira, W. F., Gilmour, J. P., Harrison, H. B., Heron S. F., Hoey, A. S., Hobbs, J. A., Hoogenboom, M. O., Kennedy, E. V., Kuo, C. Y., Lough, J. M., Lowe, R. J., Liu, G., McCulloch, M. T., Malcolm, H. A., McWilliam, M. J., Pandolfi, J. M., Pears, R. J., Pratchett, M. S., Schoepf, V., Simpson, T., Skirving, W. J., Sommer, B., Torda, G., Wachenfeld, D. R., Willis, B. L., and Wilson, S. K. (2017a). Global warming and recurrent mass bleaching of corals. Nature, 543:373-378. https://doi.org/10.1038/nature21707

Hughes, T. P., Barnes, M. L., Bellwood, D. R., Cinner, J. E., Cumming, G. S., Jackson, J. B. C., Kleypas, J., van de Leemput, I. A., Lough, J. M., Morrison, T. H., Palumbi, S. R., van Nies, E. H., and Scheffer, M. (2017b). Coral reefs in the Anthropocene. Nature, 546:82-90. https://doi.org/10.1007/978-94-017-7249-5

Hunt, C. L., Kelly, G. R., Windmill, H., Curtis-Quick, J., Conlon, H., Bodmer, M. D. V., Rogers, A. D., and Exton, D. A. (2019). Aggregating behaviour in invasive Caribbean lionfish is driven by habitat complexity. Scientific Reports, 9:783. https://doi.org/10.1038/s41598-01837459-w

Huntington, B. E., Miller, M. W., Pausch, R., and Richter, L. (2017). Facilitation in Caribbean coral reefs: high densities of staghorn coral foster greater coral condition and reef fish composition. Oecologia, 184:247-257. https://doi.org/10.1007/s00442-017-3859-7

ICES (2009). Report of the ICES-NAFO Working Group on Deep-water Ecology (WGDEC), 9-13 March 2009, ICES CM 2009\ACOM:23. 94 pp. 
Ihde, T. F., and Townsend, H. M. (2017). Accounting for multiple stressors influencing living marine resources in a complex estuarine ecosystem using an Atlantis model. Ecological Modelling, 365:1-9. https://doi.org/10.1016/j.ecolmodel.2017.09.010

IPCC (2014). Climate Change 2014: Synthesis Report. Contribution of Working Groups I, II and III to the Fifth Assessment Report of the Intergovernmental Panel on Climate Change [Core Writing Team, R.K. Pachauri and L.A. Meyer (eds.)]. IPCC, Geneva, Switzerland, pp 151.

Jackson, J. B., and Buss, L. (1975). Alleopathy and spatial competition among coral reef invertebrates. Proceedings of the National Academy of Sciences, 72(12):5160-5163. https://doi.org/10.1073/pnas.72.12.5160

Johns, K. A., Emslie, M. J., Hoey, A. S., Osborne, K., Jonker, M. J., and Cheal, A. J. (2018). Macroalgal feedbacks and substrate properties maintain a coral reef regime shift. Ecosphere, 9(7): 02349 .

Johnson, D. W. (2007). Habitat complexity modifies post-settlement mortality and recruitment dynamics of a marine fish. Ecology, 88(7):1716-1725.

Jones, G. P., Mccormick, M. I., Srinivasan, M., and Eagle, J. V. (2004). Coral decline threatens fish biodiversity in marine reserves. Proceedings of the National Academy of Sciences, 101(21):8251-8253.

Jopp, F., Reuter, H., and Breckling, B. (Eds) (2011). Modelling complex ecological dynamics. Springer-Verlag, Berlin Heidelberg, 397 pp.

Jouffray, J.-B., Nystrom, M., Norstrom, A. V., Williams, I. D., Wedding, L. M., Kittinger, J. N., and Williams, G. J. (2015). Identifying multiple coral reef regimes and their drivers across the Hawaiian archipelago. Philosophical Transactions of the Royal Society B: Biological Sciences, 370:20130268. https://doi.org/10.1098/rstb.2013.0268

Kayal, M., Vercelloni, J., Lison de Loma, T., Bosserelle, P., Chancerelle, Y., Geoffroy, S., Stievenart, C., Michonneau, F., Penin, L., Planes, S., and Adjeroud, M. (2012). Predator crownof-thorns starfish (Acanthaster planci) outbreak, mass mortality of corals, and cascading effects on reef fish and benthic communities. PLoS ONE 7(10):e47363.

Kelaher, B. P., and Castilla, J. C. (2005). Habitat characteristics influence macrofaunal communities in coralline turf more than mesoscale coastal upwelling on the coast of Northern 
Chile. Estuarine, Coastal and Shelf Science, 63:155-165. https://doi.org/10.1016/j.ecss.2004.10.017

Kelmo, F., Bell, J. J., Moraes, S. S., Gomes, R. D. C. T., Mariano-Neto, E., and Attrill, M. J. (2014). Differential responses of emergent intertidal coral reef fauna to a large-scale El-Niño southern oscillation event: Sponge and coral resilience. PLoS ONE, 9(3):e93209. https://doi.org/10.1371/journal.pone.0093209

Kelmo, F., Bell, J. J., and Attrill, M. J. (2013). Tolerance of sponge assemblages to temperature anomalies: Resilience and proliferation of sponges following the 1997-8 El-Niño Southern Oscillation. PLoS ONE, 88(10): e76441. doi:10.1371/journal.pone.0076441

Kenchington, E., Power, D., and Koen-Alonso, M. (2013). Associations of demersal fish with sponge grounds on the continental slopes of the northwest Atlantic. Marine Ecology Progress Series, 477:217-230. https://doi.org/10.3354/meps10127

Kerry, J. T., and Bellwood, D. R. (2015a). Do tabular corals constitute keystone structures for fishes on coral reefs? Coral Reefs, 34:41-50. https://doi.org/10.1007/s00338-014-1232-4

Kerry, J. T., and Bellwood, D. R. (2015b). The functional role of tabular structures for large reef fishes: avoiding predators or solar irradiance? Coral Reefs, 34:693-702. https://doi.org/10.1007/s00338-015-1275-1

Kerry, J. T., and Bellwood, D. R. (2012). The effect of coral morphology on shelter selection by coral reef fishes. Coral Reefs, 31:415-424. https://doi.org/10.1007/s00338-011-0859-7

Kleypas, J. A., Buddemeier, R. W., and Gattuso, J.-P. (2001). The future of coral reefs in an age of global change. International Journal of Earth Sciences, 90:426-437. https://doi.org/10.1007/s005310000125

Klumpp, D. W., and McKinnon, A. D. (1989). Temporal and spatial patterns in primary production of a coral-reef epilithic algal community. Journal of Experimental Marine Biology and Ecology, 131:1-22. https://doi.org/10.1016/0022-0981(89)90008-7

Knapp, I. S. S., Williams, G. J., and Bell, J. J. (2016). Temporal dynamics and persistence of sponge assemblages in a Central Pacific atoll lagoon. Marine Ecology, 37(5):1147-1153. https://doi.org/10.1111/maec.12303

Knapp, I. S. S., Williams, G. J., Carballo, J. L., Cruz-Barraza, J. A., Gardner, J. P. A., and Bell, J. J. (2013). Restriction of sponges to an atoll lagoon as a result of reduced environmental 
quality.

Marine

Pollution

Bulletin,

66:209-220.

https://doi.org/10.1016/j.marpolbul.2012.08.017

Kohler, K. E., and Gill, S. M. (2006). Coral Point Count with Excel extensions (CPCe): A Visual Basic program for the determination of coral and substrate coverage using random point count methodology. Computers and Geosciences, 32(9):1259-1269. https://doi.org/10.1016/j.cageo.2005.11.009

Koopmans, M., Martens, D., and Wijffels, R. H. (2010). Growth efficiency and carbon balance for the sponge Haliclona oculata. Marine Biotechnology, 12(3):340-349. https://doi.org/10.1007/s10126-009-9228-8

Koukouras, A., Russo, A., Voultsiadou-Koukoura, E., Arvanitidis, C., and Stefanidou, D. (1996). Macrofauna associated with sponge species of different morphology. Marine Ecology, 17(4):569-582. https://doi.org/10.1111/j.1439-0485.1996.tb00418.x

Lamy, T., Galzin, R., Kulbicki, M., Lison de Loma, T., and Claudet, J. (2016). Three decades of recurrent declines and recoveries in corals belie ongoing change in fish assemblages. Coral Reefs, 35:293-302.

Lapointe, B. E., Brewton, R. A., Herren, L. W., Porter, J. W., and Hu, C. (2019). Nitrogen enrichment, altered stoichiometry, and coral reef decline at Looe Key, Florida Keys, USA: a 3-decade study. Marine Biology, 166:108. https://doi.org/10.1007/s00227-019-3538-9

Lavy, A., Eyal, G., Neal, B., Keren, R., Loya, Y., and Ilan, M. (2015). A quick, easy and nonintrusive method for underwater volume and surface area evaluation of benthic organisms by 3D computer modelling. Methods in Ecology and Evolution, 6:521-531. https://doi.org/10.1111/2041-210X.12331

Lawler, M., and Osborn, J. (2008). Photogrammetric classification of sponge morphometric diversity. In 14th Australian Remote Sensing and Photogrammetry Conference. Darwin.

Legendre, P. and L. Legendre. (2012). Numerical ecology, 3rd English edition. Elsevier Science BV, Amsterdam. 990 pp.

Lenth, R. (2019). emmeans: Estimated Marginal Means, aka Least-Squares Means. R package version 1.3.3. https://CRAN.R-project.org/package=emmeans 
Leon, J. X., Roelfsema, C. M., Saunders, M. I., and Phinn, S. R. (2015). Measuring coral reef terrain roughness using 'Structure-from-Motion' close-range photogrammetry. Geomorphology, 242:21-28. https://doi.org/10.1016/j.geomorph.2015.01.030

Lesser, M. P. (2006). Benthic-pelagic coupling on coral reefs: Feeding and growth of Caribbean sponges. Journal of Experimental Marine Biology and Ecology, 328:277-288. https://doi.org/10.1016/j.jembe.2005.07.010

Lesser, M. P., and Slattery, M. (2013). Ecology of Caribbean sponges: Are top-down or bottom-up processes more important? PLoS ONE 8(11):e79799. https://doi.org/10.1371/journal.pone.0079799

Lesser, M. P., Fiore, C., Slattery, M., and Zaneveld, J. (2016). Climate change stressors destabilize the microbiome of the Caribbean barrel sponge, Xestospongia muta. Journal of Experimental Marine Biology and Ecology, 475:11-18. https://doi.org/10.1016/j.jembe.2015.11.004

Levins, R. (1974). The qualitative analysis of partially specified systems. Annals New York Academy of Sciences.

Levins, R. (1968). Evolution in changing environments. Some theoretical explorations. Princeton: Princeton University Press. https://doi.org/10.1126/science.167.3924.1478

Li, C., Chen, J-Y, and Hua, T-E. (1998). Precambrian sponges with cellular structures. Science, 279(5352):879-882. https://doi.org/10.1126/science.279.5352.879

Lindahl, U., Öhman, M. C., and Schelten, C. K. (2001). The 1997/1998 mass mortality of corals: Effects on fish communities on a Tanzanian coral reef. Marine Pollution Bulletin, 42(2):127-131. https://doi.org/10.1016/S0025-326X(00)00167-3

Link, J. S., Fulton, E. A., and Gamble, R. J. (2010). The northeast US application of ATLANTIS: A full system model exploring marine ecosystem dynamics in a living marine resource management context. Progress in Oceanography, 87:214-234. https://doi.org/10.1016/j.pocean.2010.09.020

Logofet, D.O. (1993). Matrices and graphs: stability problems in mathematical ecology. CRC, Boca Raton, 320 pp. 
Loh, T-L., and Pawlik, J. R. (2014). Chemical defenses and resource trade-offs structure sponge communities on Caribbean coral reefs. Proceedings of the National Academy of Sciences, 111(11):4151-4156. https://doi.org/10.1073/pnas.1321626111

Loh, T-L, and Pawlik, J. R. (2012). Friend or foe? No evidence that association with the sponge Mycale laevis provides a benefit to corals of the genus Montastraea. Marine Ecology Progress Series, 465:111-117.

Loh, T.-L., McMurray, S. E., Henkel, T. P., Vicente, J., and Pawlik, J. R. (2015). Indirect effects of overfishing on Caribbean reefs: sponges overgrow reef-building corals. PeerJ, 3:e901. https://doi.org/10.7717/peerj.901

Lopez-Acosta, M., Leynaert, A., Grall, J., and Maldonado, M. (2018) Silicon consumption kinetics by marine sponges: an assessment of their role at the ecosystem level. Limnology and Oceanography 63:2508-22.

López-Legentil, S., Song, B., McMurray, S. E., and Pawlik, J. R. (2008). Bleaching and stress in coral reef ecosystems: hsp70 expression by the giant barrel sponge Xestospongia muta. Molecular Ecology, 17:1840-1849. https://doi.org/10.1111/j.1365-294X.2008.03667.x

López-Victoria, M., and Zea, S. (2005). Current trends of space occupation by encrusting excavating sponges on Colombian coral reefs. Marine Ecology, 26:33-41.

López-Victoria, M., Zea, S., and Weil, E. (2006). Competition for space between encrusting excavating Caribbean sponges and other coral reef organisms. Marine Ecology Progress Series, 312:113-121. https://doi.org/10.3354/meps312113

Ma, M. (2005). Species richness vs evenness: Independent relationship and different responses to edaphic factors. Oikos, 111(1):192-198. https://doi.org/10.1111/j.00301299.2005.13049.x

MacArthur, R. H., and MacArthur, J. W. (1961). On bird species diversity. Ecology, 42(3):594-598.

MacGeanchy, J. K. (1977). Factors controlling sponge boring in Barbados reef corals. Proceedings of the 3rd International Coral Reef Symposium 2, 477-483.

Magel, J. M. T., Burns, J. H. R., Gates, R. D., and Baum, J. K. (2019). Effects of bleachingassociated mass coral mortality on reef structural complexity across a gradient of local disturbance. Scientific Reports, 9:2512. https://doi.org/10.1038/s41598-018-37713-1 
Maldonado, M. (2006). The ecology of the sponge larva. Canadian Journal of Zoology, 84:175-194.

Maldonado, M., Aguilar, R., Bannister, R. J., Bell, J. J., Conway, K. W., Dayton, P. K., ... Young, C. M. (2016). Sponge grounds as key marine habitats: A synthetic review of types, structure, functional roles, and conservation concerns. In S. Rossi, L. Bramanti, A. Gori, and C. O. S. del Valle (Eds.), Marine Animal Forests: The Ecology of Benthic Biodiversity Hotspots. Switzerland: Springer. https://doi.org/10.1007/978-3-319-17001-5_17-2

Maldonado, M., Ribes, M., and van Duyl, F. C. (2012). Nutrient fluxes through sponges. Biology, budgets, and ecological implications. Advances in Marine Biology, 62:113-182. https://doi.org/10.1016/B978-0-12-394283-8.00003-5

Maldonado, M., Navarro, L., Grasa, A., Gonzalez, A., and Vaquerizo, I. (2011). Silicon uptake by sponges: A twist to understanding nutrient cycling on continental margins. Scientific Reports, 1:30. https://doi.org/10.1038/srep00030

Maldonado, M., Sanchez-Tocino, L., and Navarro, C. (2010). Recurrent disease outbreaks in corneous demosponges of the genus Ircinia: Epidemic incidence and defense mechanisms. Marine Biology, 157(7):1577-1590. https://doi.org/10.1007/s00227-010-1431-7

Maldonado, M., Carmona, M. C., Velásquez, Z., Puig, A., Cruzado, A., López, A., and Young, C. M. (2005). Siliceous sponges as a silicon sink: An overlooked aspect of the benthopelagic coupling in the marine silicon cycle. Limnology and Oceanography, 50(3):799809. https://doi.org/10.4319/lo.2005.50.3.0799

Manconi, R., and Pronzato, R. (2008). Global diversity of sponges (Porifera: Spongillina) in freshwater. Hydrobiologia, 595:27-33. https://doi.org/10.1007/s10750-007-9000-x

Marlow, J., Schönberg, C. H. L., Davy, S. K., Haris, A., Jompa, J., and Bell, J. J. (2018a). Bioeroding sponge assemblages: the importance of substrate availability and sediment. Journal of the Marine Biological Association of the United Kingdom, 1-16.

Marlow, J., Davy, S. K., Shaffer, M., Haris, A., and Bell, J. J. (2018b). Bleaching and recovery of a phototrophic bioeroding sponge. Coral Reefs, 37:565-570.

Marlow, J., Smith, D. J., Werorilang, S., and Bell, J. J. (2018c). Sedimentation limits the erosion rate of a bioeroding sponge. Marine Ecology, 39:e12483. https://doi.org/10.1111/maec.12483 
Marlow, J., Davy, S. K., Haris, A., and Bell, J. J. (2018d). Photoacclimation to lightlimitation in a clionaid sponge; implications for understanding sponge bioerosion on turbid reefs. Marine Pollution $\quad$ Bulletin, 135:466-474. https://doi.org/10.1016/j.marpolbul.2018.07.049

Marshall, K. N., Kaplan, I. C., Hodgson, E. E., Hermann, A., Busch, D. S., McElhany, P., Essington, T. E., Harvey, C. J., and Fulton, E. A. (2017). Risks of ocean acidification in the California Current food web and fisheries: ecosystem model projections. Global Change Biology, 23:1525-1539. https://doi.org/10.1111/gcb.13594

Massaro, A. J., Weisz, J. B., Hill, M. S., and Webster, N. S. (2012). Behavioral and morphological changes caused by thermal stress in the Great Barrier Reef sponge Rhopaloeides odorabile. Journal of Experimental Marine Biology and Ecology, 416-417:55-60. https://doi.org/10.1016/j.jembe.2012.02.008

McCain, C., Szewczyk, T., and Bracy Knight, K. (2016). Population variability complicates the accurate detection of climate change responses. Global Change Biology, 22:2081-2093.

McClintock, J. B., Amsler, C. D., Baker, B. J., and van Soest, R. W. M. (2005). Ecology of antarctic marine sponges: an overview. Integrative and Comparative Biology, 45(2):359-368. https://doi.org/10.1093/icb/45.2.359

McGrath, E. C., Woods, L., Jompa, J., Haris, A., and Bell, J. J. (2018). Growth and longevity in giant barrel sponges: Redwoods of the reef or Pines in the Indo-Pacific? Scientific Reports, 8:15317. https://doi.org/10.1038/s41598-018-33294-1

McGrath, E. C., Smith, D. J., Jompa, J., and Bell, J. J. (2017). Adaptive mechanisms and physiological effects of suspended and settled sediment on barrel sponges. Journal of Experimental Marine Biology and Ecology, 496:74-83. https://doi.org/10.1016/j.jembe.2017.07.013

Mclean, E. L., Rützler, K., and Pooler, P. S. (2015). Competing for space: factors that lead to sponge overgrowth when interacting with octocoral. Open Journal of Marine Science, 5:6480 .

McMellor, S., and Smith, D. J. (2010). Coral reefs of the Wakatobi: abundance and biodiversity. In Clifton, J., Unsworth, R. K. F., and Smith, D. J. (Eds.), Marine research and conservation in the Coral Triangle: the Wakatobi National Park (pp. 11-26). Environmental Science, Engineering and Technology Series. Nova Publishers, New York, 274 pp. 
McMurray, S. E., Stubler, A. D., Erwin, P. M., Finelli, C. M., and Pawlik, J. R. (2018). A test of the sponge-loop hypothesis for emergent Caribbean reef sponges. Marine Ecology Progress Series, 588:1-14. https://doi.org/10.3354/meps12466

McMurray, S. E., Pawlik, J. R., and Finelli, C. M. (2017). Demography alters carbon flux for a dominant benthic suspension feeder, the giant barrel sponge, on Conch Reef, Florida Keys. Functional Ecology, 31:2188-2198. https://doi.org/10.1111/1365-2435.12908

McMurray, S. E., Johnson, Z. I., Hunt, D. E., Pawlik, J. R., and Finelli, C. M. (2016). Selective feeding by the giant barrel sponge enhances foraging efficiency. Limnology and Oceanography, 61:1271-1286. https://doi.org/10.1002/lno.10287

McMurray, S. E., Blum, J. E., Leichter, J. J., and Pawlik, J. R. (2011). Bleaching of the giant barrel sponge Xestospongia muta in the Florida Keys. Limnology and Oceanography, 56(6):2243-2250. https://doi.org/10.4319/lo.2011.56.6.2243

McMurray, S. E., Henkel, T. P., and Pawlik, J. R. (2010). Demographics of increasing populations of the giant barrel sponge Xestospongia muta in the Florida Keys Stable. Ecology, 91(2):560-570

McMurray, S. E., Blum, J. E., and Pawlik, J. R. (2008). Redwood of the reef: Growth and age of the giant barrel sponge Xestospongia muta in the Florida Keys. Marine Biology, 155:159-171. https://doi.org/10.1007/s00227-008-1014-z

Meissner, K. J., Lippmann, T., and Sen Gupta, A. (2012). Large-scale stress factors affecting coral reefs: Open ocean sea surface temperature and surface seawater aragonite saturation over the next 400 years. Coral Reefs, 31:309-319.

Mercado-Molina, A. E., and Yoshioka, P. M. (2009). Relationships between water motion and size-specific survivorship and growth of the demosponge Amphimedon compressa. Journal of Experimental Marine Biology and Ecology, 375:51-56. https://doi.org/10.1016/j.jembe.2009.05.003

Messmer, V., Jones, G. P., Munday, P. L., Holbrook, S. J., Schmitt, R. J., and Brooks, A. J. (2011). Habitat biodiversity as a determinant of fish community structure on coral reefs. Ecology, 92(12):2285-2298. https://doi.org/10.1890/11-0037.1

Meylan, A. (1988). Spongivory in hawksbill turtles: A diet of glass. Science, 239(4838):393395. 
Mills, D. B., Ward, L. M., Jones, C., Sweeten, B., Forth, M., Treusch, A. H., and Canfield, D. E. (2014). Oxygen requirements of the earliest animals. Proceedings of the National Academy of Sciences, 111(11):4168-4172. https://doi.org/10.1073/pnas.1400547111

Mokany, K., Thomson, J. J., Lynch, A. J. J., Jordan, G. J., and Ferrier, S. (2015). Linking changes in community composition and function under climate change. Ecological Applications, 25(8):2132-2141.

Monismith, S. G., Rogers, J. S., Koweek, D., and Dunbar, R. B. (2015). Frictional wave dissipation on a remarkably rough reef. Geophysical Research Letters, 42:4063-4071. https://doi.org/10.1002/2015GL063804

Morrow, C., and Cárdenas, P. (2015). Proposal for a revised classification of the Demospongiae (Porifera). Frontiers in Zoology, 12:7. https://doi.org/10.1186/s12983-0150099-8

Morrow, K. M., Bourne, D. G., Humphrey, C., Botté, E. S., Laffy, P., Zaneveld, J., Uthicke, S., Fabricius, K. E., and Webster, N. S. (2015). Natural volcanic $\mathrm{CO}_{2}$ seeps reveal future trajectories for host-microbial associations in corals and sponges. The ISME Journal, 9:894908. https://doi.org/10.1038/ismej.2014.188

Mueller, B., de Goeij, J. M., Vermeij, M. J. A., Mulders, Y., van Der Ent, E., Ribes, M., and van Duyl, F. C. (2014). Natural diet of coral-excavating sponges consists mainly of dissolved $\begin{array}{llll}\text { organic carbon } \quad \text { (DOC). } & \text { ONE, } & \text { 9 (2):e90152. }\end{array}$ https://doi.org/10.1371/journal.pone.0090152

Mumby, P. J., and Steneck, R. S. (2018). Paradigm lost: Dynamic nutrients and missing detritus on coral reefs. BioScience, 68(7):487-495. https://doi.org/10.1093/biosci/biy055

Mumby, P. J., and Van Woesik, R. (2014). Consequences of ecological, evolutionary and biogeochemical uncertainty for coral reef responses to climatic stress. Current Biology, 24(10):R413-R423. https://doi.org/10.1016/j.cub.2014.04.029

Mumby, P. J., and Steneck, R. S. (2008). Coral reef management and conservation in light of rapidly evolving ecological paradigms. Trends in Ecology and Evolution, 23(10):555-563. https://doi.org/10.1016/j.tree.2008.06.011

Mumby, P. J., Hastings, A., and Edwards, H. J. (2007). Thresholds and the resilience of Caribbean coral reefs. Nature, 450(7166):98-101. https://doi.org/10.1038/nature06252 
Murtagh, F., and Legendre, P. (2014). Ward's hierarchical agglomerative clustering method: Which algorithms implement ward's criterion? Journal of Classification, 31(3):274-295. https://doi.org/10.1007/s00357-014-9161-z

Nagelkerken, I., Van Der Velde, G., Wartenbergh, S.L.J., Nugues, M.M. and Pratchett, M.S. (2009) Cryptic dietary components reduce dietary overlap among sympatric butterflyfishes (Chaetodontidae). Journal of Fish Biology 75:1123-1143.

Nash, K. L., Graham, N. A. J., Wilson, S. K., and Bellwood, D. R. (2013). Cross-scale habitat structure drives fish body size distributions on coral reefs. Ecosystems, 16:478-490. https://doi.org/10.1007/s10021-012-9625-0

Negri, A. P., Webster, N. S., Hill, R. T., and Heyward, A. J. (2001). Metamorphosis of broadcast spawning corals in response to bacteria isolated from crustose algae. Marine Ecology Progress Series, 223:121-131.

Newman, S. P., Meesters, E. H., Dryden, C. S., Williams, S. M., Sanchez, C., Mumby, P. J., and Polunin, N. V. C. (2015). Reef flattening effects on total richness and species responses in the Caribbean. Journal of Animal Ecology, 84:1678-1689. https://doi.org/10.1111/13652656.12429

NOAA Coral Reef Watch. 2000, updated twice-weekly. NOAA Coral Reef Watch 50-km Satellite Virtual Station Time Series Data for Wakatobi, Jan. 1, 2005-Dec. 31, 2017. Silver Spring, Maryland, USA: NOAA Coral Reef Watch. Data set accessed 2018-02-14 at https://coralreefwatch.noaa.gov/satellite/vs/docs/list_vs_group_latlon_201103.php.

Nordlund, L. M., Unsworth, R. K. F., Gullström, M., and Cullen-Unsworth, L. C. (2018). Global significance of seagrass fishery activity. Fish and Fisheries, 19(3):399-412. https://doi.org/10.1111/faf.12259

Norström, A. V., Nyström, M., Lokrantz, J., and Folke, C. (2009). Alternative states on coral reefs: Beyond coral-macroalgal phase shifts. Marine Ecology Progress Series, 376:293-306. https://doi.org/10.3354/meps07815

Nye, J. A., Gamble, R. J., and Link, J. S. (2013). The relative impact of warming and removing top predators on the Northeast US large marine biotic community. Ecological Modelling, 264:157-168. https://doi.org/10.1016/j.ecolmodel.2012.08.019 
O'Brien, J. M., and Scheibling, R. E. (2018). Turf wars: Competition between foundation and turf-forming species on temperate and tropical reefs and its role in regime shifts. Marine Ecology Progress Series, 590:1-17.

O'Connor, N. A. (1991). The effects of habitat complexity on the macroinvertebrates colonising wood substrates in a lowland stream. Oecologia, 85:504-512.

Oksanen, J., Blanchet, F. G., Friendly, M., Kindt, R., Legendre, P., McGlinn, D., Minchin, P. R., O'Hara, R. B., Simpson, G. L., Solymos, P., Stevens, M. H. H., Szoecs, E., and Wagner, H. (2019). vegan: Community Ecology Package. Retrieved from https://cran.rproject.org/package=vegan

Opitz, S. (1996). Trophic Interactions in Caribbean Coral Reefs. International Center for Living Aquatic Resource Management Technical Reports, 43, 341 pp.

Ortega-Cisneros, K., Cochrane, K. L., Fulton, E. A., Gorton, R., and Popova, E. (2018). Evaluating the effects of climate change in the southern Benguela upwelling system using the Atlantis modelling framework. Fisheries Oceanography, 27(5):489-503. https://doi.org/10.1111/fog.12268

Orr, J. C., Fabry, V. J., Aumont, O., Bopp, L., Feely, R. A., Doney, S. C., Gnanadesikan, A., Gruber, N., Ishida, A., Joos, F., Key, R. M., Lindsay, K., Maier-Reimer, E., Matear, R., Monfray, P., Mouchet, A., Najjar, R. G., Plattner, G.-K., Rodgers, K. B., Sabine, C. L., Sarmiento, J. L., Schlitzer, R., Slater, R. D., Totterdell, I. J., Weirig, M-F., Yamanaka, Y., and Yool, A. (2005). Anthropogenic ocean acidification over the twenty-first century and its impact on calcifying organisms. Nature, 437(7059), 681-686. https://doi.org/10.1038/nature04095

Padua, A., Leocorny, P., Custódio, M. R., and Klautau, M. (2016). Fragmentation, fusion, and genetic homogeneity in a calcareous sponge (Porifera, Calcarea). Journal of Experimental Zoology Part A: Ecological Genetics and Physiology, 325A:294-303.

Pang, R. K. (1973). Ecology of some Jamaican excavating sponges. Bulletin of Marine Science, 23(2):227-243.

Papatheodoulou, M., Jimenez, C., Petrou, A., and Thasitis, I. (2019). Endobiotic communities of marine sponges in Cyprus (Levantine Sea). Heliyon, 5:e01392. https://doi.org/10.1016/j.heliyon.2019.e01392 
Pawlik, J. R. (2011). The chemical ecology of sponges on Caribbean reefs: Natural products shape natural systems. BioScience, 61:888-898. https://doi.org/10.1525/bio.2011.61.11.8

Pawlik, J. R., and McMurray, S. E. (2020). The emerging ecological and biogeochemical importance of sponges on coral reefs. Annual Review of Marine Science, 12: 315-337.

Pawlik, J. R., and Deignan, L. K. (2015). Cowries graze verongid sponges on Caribbean reefs. Coral Reefs, 34:663. https://doi.org/10.1007/s00338-015-1279-x

Pawlik, J. R., Loh, T.-L., and McMurray, S. E. (2018). A review of bottom-up vs. top-down control of sponges on Caribbean fore-reefs: what's old, what's new, and future directions. PeerJ, 6:e4343. https://doi.org/10.7717/peerj.4343

Pawlik, J. R., Burkepile, D. E., and Thurber, R. V. (2016). A vicious circle? Altered carbon and nutrient cycling may explain the low resilience of Caribbean coral reefs. BioScience, 66(6):470-476. https://doi.org/10.1093/biosci/biw047

Pawlik, J. R., McMurray, S. E., Erwin, P., and Zea, S. (2015). A review of evidence for food limitation of sponges on Caribbean reefs. Marine Ecology Progress Series, 519:265-283.

Pawlik, J. R., Loh, T. L., McMurray, S. E., and Finelli, C. M. (2013). Sponge communities on Caribbean coral reefs are structured by factors that are top-down, not bottom-up. PLoS ONE 8(5):e62573. doi:10.1371/journal.pone.0062573

Pawlik, J. R, Steindler, L., Henkel, T. P., Beer, S., and Ilan, M. (2007). Chemical warfare on coral reefs : affect coral symbiosis. Limnology and Oceanography, 52(2):907-911.

Pethybridge, H. R., Weijerman, M., Perrymann, H., Audzijonyte, A., Porobic, J., McGregor, V., Girardin, R., Bulman, C., Ortega-Cisneros, K., Sinerchia, M., Hutton, T., Lozano-Montes, H., Mori, M., Novaglio, C., Fay, G., Gorton, R., and Fulton, E. (2019). Calibrating processbased marine ecosystem models: An example case using Atlantis. Ecological Modelling, 412:108822. https://doi.org/10.1016/j.ecolmodel.2019.108822

Pinheiro J, Bates D, DebRoy S, Sarkar D and R Core Team (2018) nlme: Linear and Nonlinear Mixed Effects Models. Available at: https://cran.r-project.org/package=nlme

Pitcher, C. R., Doherty, P., Arnold, P., Hooper, J., and Gribble, N. (2007). Seabed biodiversity on the continental shelf of the Great Barrier Reef World Heritage Area. AIMS/CSIRO/QM/QDPI CRC Reef Research Task Final Report. 
Plass-Johnson, J. G., Heiden, J. P., Abu, N., Lukman, M., and Teichberg, M. (2016). Experimental analysis of the effects of consumer exclusion on recruitment and succession of a coral reef system along a water quality gradient in the Spermonde Archipelago, Indonesia. Coral Reefs, 35:229-243. https://doi.org/10.1007/s00338-015-1369-9

Plass-Johnson, J. G., Ferse, S. C. A., Jompa, J., Wild, C., and Teichberg, M. (2015). Fish herbivory as key ecological function in a heavily degraded coral reef system. Limnology and Oceanography, 60:1382-1391.

Porter, J. W., and Targett, N. M. (1988). Allelochemical interactions between sponges and corals. Biological Bulletin, 175:230-239. https://doi.org/10.2307/1541563

Pörtner, H. O., Langenbuch, M., and Reipschläger, A. (2004). Biological impact of elevated ocean $\mathrm{CO}_{2}$ concentrations: Lessons from animal physiology and earth history. Journal of Oceanography, 60:705-718. https://doi.org/10.1007/s10872-004-5763-0

Powell, A., Jones, T., Smith, D. J., Jompa, J., and Bell, J. J. (2015). Spongivory in the Wakatobi Marine National Park, Southeast Sulawesi, Indonesia. Pacific Science, 69(4):487508. https://doi.org/10.2984/69.4.5

Powell, A., Smith, D. J., Hepburn, L. J., Jones, T., Berman, J., Jompa, J., and Bell, J. J. (2014). Reduced diversity and high sponge abundance on a sedimented indo-pacific reef system: Implications for future changes in environmental quality. PLoS ONE, 9(1):e85253. https://doi.org/10.1371/journal.pone.0085253

Powell, A. L., Hepburn, L. J., Smith, D. J., and Bell, J. J. (2010). Patterns of sponge abundance across a gradient of habitat quality in the Wakatobi Marine National Park, Indonesia. The Open Marine Biology Journal, 4:31-38.

Puccia, C.J., Levins, R. (1985). Qualitative modelling of complex systems. An introduction to loop analysis and time averaging. Harvard University Press, Cambridge, Massachusetts, and London, England. http://doi.org/10.4159/harvard.9780674435070

QGIS Development Team (2018). QGIS Geographic Information System. Open Source Geospatial Foundation Project. http://qgis.osgeo.org

Qian, P. Y., Lau, S. C. K., Dahms, H. U., Dobretsov, S., and Harder, T. (2007). Marine biofilms as mediators of colonization by marine macroorganisms: Implications for antifouling and aquaculture. Marine Biotechnologies, 9:399-410. 
R Core Team (2019) R: A language and environment for statistical computing. Vienna, Austria: R Foundation for Statistical Computing. Available at: https://www.r-project.org/

Ramsby, B. D., Hoogenboom, M. O., Smith, H. A., Whalan, S., and Webster, N. S. (2018). The bioeroding sponge Cliona orientalis will not tolerate future projected ocean warming. Scientific Reports, 8:8302. https://doi.org/10.1038/s41598-018-26535-w

Ramsby, B. D., Hoogenboom, M. O., Whalan, S., Webster, N. S., and Thompson, A. (2017). A decadal analysis of bioeroding sponge cover on the inshore Great Barrier Reef. Scientific Reports, 7:2706.

Reichert, J., Backes, A. R., Schubert, P., and Wilke, T. (2017). The power of 3D fractal dimensions for comparative shape and structural complexity analyses of irregularly shaped organisms. Methods in Ecology and Evolution, 8:1650-1658. https://doi.org/10.1111/2041210X.12829

Reiswig, H. M. (1975). Bacteria as food for temperate-water marine sponges. Canadian Journal of Zoology, 53(5):582-589. https://doi.org/10.1139/z75-072

Reiswig, H. M. (1973). Population dynamics of three Jamaican Demospongiae. Bulletin of Marine Science, 23:191-226.

Reiswig, H. M. (1971a). Particle feeding in natural populations of three marine demosponges. Biological Bulletin, 141(3), 568-591.

Reiswig, H. M. (1971b). In situ pumping activities of tropical Demospongiae. Marine Biology, 9(1):38-50. https://doi.org/10.1007/BF00348816

Ribeiro, B., Padua, A., Paiva, P. C., Custódio, M. R., and Klautau, M. (2018). Exploitation of micro refuges and epibiosis: Survival strategies of a calcareous sponge. Journal of the Marine Biological Association of the United Kingdom, 98(3):495-503. https://doi.org/10.1017/S002531541600151X

Ribes, M., Coma, R., and Gili, J-M. (1999). Natural diet and grazing rate of the temperate sponge Dysidea avara (Demospongiae, Dendroceratida) throughout an annual cycle. Marine Ecology Progress Series, 176:179-190.

Ribes, M., Atkinson, M. J., and Kinzie Iii, R. A. (2005). Sponges and ascidians control removal of particulate organic nitrogen from coral reef water. Limnology and Oceanography, 50(5):1480-1489. 
Richardson, L. E., Graham, N. A. J., and Hoey, A. S. (2017). Cross-scale habitat structure driven by coral species composition on tropical reefs. Scientific Reports, 7:7557. https://doi.org/10.1038/s41598-017-08109-4

Rickert, D., Schlüter, M., and Wallmann, K. (2002). Dissolution kinetics of biogenic silica from the water column to the sediments. Geochimica et Cosmochimica Acta, 66(3):439-455. https://doi.org/10.1016/S0016-7037(01)00757-8

Rix, L., de Goeij, J. M., van Oevelen, D., Struck, U., Al-Horani, F. A., Wild, C., and Naumann, M. S. (2018). Reef sponges facilitate the transfer of coral-derived organic matter to their associated fauna via the sponge loop. Marine Ecology Progress Series, 589:85-96. https://doi.org/10.3354/meps 12443

Rix, L., de Goeij, J. M., van Oevelen, D., Struck, U., Al-Horani, F. A., Wild, C., and Naumann, M. S. (2017). Differential recycling of coral and algal dissolved organic matter via the sponge loop. Functional Ecology, 31:778-789. https://doi.org/10.1111/1365-2435.12758

Rix, L., de Goeij, J. M., Mueller, C. E., Struck, U., Middelburg, J. J., van Duyl, F. C., AlHorani, F. A., Wild, C., Naumann, M. S., and van Oevelen, D. (2016). Coral mucus fuels the sponge loop in warm- and cold-water coral reef ecosystems. Scientific Reports, 6:18715. https://doi.org/10.1038/srep18715

Roberts, C. M., McClean, C. J., Veron, J. E. N., Hawkins, J. P., Allen, G. R., McAllister, D. E., Mittermeier, C. G., Schueler, F. W., Spalding, M., Wells, F., Vynne, C., and Werner, T. B. (2002). Marine biodiversity hotspots and conservation priorities for tropical reefs. Science 295(5558):1280-1285.

Roff, G., and Mumby, P. J. (2012). Global disparity in the resilience of coral reefs. Trends in Ecology and Evolution, 27(7):404-413. https://doi.org/10.1016/j.tree.2012.04.007

Rogers, A., Blanchard, J. L., and Mumby, P. J. (2018). Fisheries productivity under progressive coral reef degradation. Journal of Applied Ecology, 55:1041-1049. https://doi.org/10.1111/1365-2664.13051

Rogers, A., Blanchard, J. L., and Mumby, P. J. (2014). Vulnerability of coral reef fisheries to a loss of structural complexity. Current Biology, 24:1000-1005. https://doi.org/10.1016/j.cub.2014.03.026 
Rogers, C. S., Fitz, H. C. III, Gilnack, M., Beets, J., and Hardin, J. (1984). Scleractinian coral recruitment patterns at Salt River Submarine Canyon, St. Croix, US Virgin Islands. Coral Reefs 3:69-76.

Rossi, G., Montori, S., Cerrano, C., and Calcinai, B. (2015). The coral killing sponge Chalinula nematifera (Porifera: Haplosclerida) along the eastern coast of Sulawesi Island (Indonesia). Italian Journal of Zoology, 82(1):143-148. https://doi.org/10.1080/11250003.2014.994046

Roth, S. K., Powell, A., Smith, D. J., Roth, F., and Schierwater, B. (2018). The highly competitive ascidian Didemnum sp. threatens coral reef communities in the Wakatobi Marine National Park, Southeast Sulawesi, Indonesia. Regional Studies in Marine Science, 24:48-54.

Rovellini, A., Dunn, M. R., Fulton, E. A., Webster, N. S., Smith, D. J., Jompa, J., Haris, A., Berman, J., and Bell, J. J. (2019). Decadal variability in sponge abundance and biodiversity on an Indo-Pacific coral reef. Marine Ecology Progress Series, 620:63-76. https://doi.org/10.3354/meps 12968

Rützler, K. (2004). Sponges on coral reefs: a community shaped by competitive cooperation. Bollettino dei Musei e degli Istituti Biologici dell’Universitá di Genova. 68:85-148.

Rützler, K. (1975). The role of burrowing sponges in bioerosion. Oecologia, 19(3):203-216. https://doi.org/10.1007/BF00345306

Rützler, K. (1970). Spatial competition among Porifera: Solution by epizoism. Oecologia, 5(2):85-95. https://doi.org/10.1007/BF00347624

Ryer, C. H., Stoner, A. W., and Titgen, R. H. (2004). Behavioral mechanisms underlying the refuge value of benthic habitat structure for two flatfishes with differing anti-predator strategies. Marine Ecology Progress Series, 268:231-243. https://doi.org/10.3354/meps268231

Sammarco, P., and Risk, M. (1990). Large-scale patterns in internal bioerosion of Porites: cross continental shelf trends on the Great Barrier Reef. Marine Ecology Progress Series, 59:145-156. https://doi.org/10.3354/meps059145

Sano, M. (1989). Feeding habits of Japanese butterflyfishes (Chaetodontidae). Environmental Biology of Fishes, 25:195-203.

Sarà, M. (1970). Competition and cooperation in sponge populations. Symposium of the Zoological Society of London, 25:273-284. 
Sawall, Y., Jompa, J., Litaay, M., Maddusila, A., and Richter, C. (2013). Coral recruitment and potential recovery of eutrophied and blast fishing impacted reefs in Spermonde Archipelago, Indonesia. Marine Pollution Bulletin, 74:374-382. https://doi.org/10.1016/j.marpolbul.2013.06.022

Scharf, F. S., Manderson, J. P., and Fabrizio, M. C. (2006). The effects of seafloor habitat complexity on survival of juvenile fishes: Species-specific interactions with structural refuge. Journal of Experimental Marine Biology and Ecology, 335:167-176. https://doi.org/10.1016/j.jembe.2006.03.018

Scheffer, M., Carpenter, S., Foley, J. A., Folke, C., and Walker, B. (2001). Catastrophic shifts in ecosystems. Nature, 413(6856):591-596.

Schils, T. (2012). Episodic eruptions of volcanic ash trigger a reversible cascade of nuisance species outbreaks in pristine coral habitats. PLoS ONE, 7(10):e46639. https://doi.org/10.1371/journal.pone.0046639

Schönberg, C. H. L., and Suwa, R. (2007). Why bioeroding sponges are better hosts for symbiotic dinoflagellates than many corals. In: Custódio M.R., Hajdu E., Lôbo-Hajdu G., Muricy G. (Eds), Porifera Research: Biodiversity, Innovation and Sustainability. Proceedings of the 7th International Sponge Symposium, Buzios, Brazil. Museu Nacional, Rio de Janeiro: $569-580$.

Schönberg, C. H. L., Fang, J. K.-H., Carreiro-Silva, M., Tribollet, A., and Wisshak, M. (2017). Bioerosion: the other ocean acidification problem. ICES Journal of Marine Science, 74(4):895-925. https://doi.org/10.1093/icesjms/fsw254

Schönberg, C. H. L., Fang, J. K.-H., and Carballo, J. L. (2017). Bioeroding Sponges and the Future of Coral Reefs. In James J. Bell and J. L. Carballo (Eds.), Climate Change, Ocean Acidification and Sponges (pp. 179-372). Springer International Publishing. https://doi.org/10.1007/978-3-319-59008-0

Searle, S.R. (1966). Matrix algebra for the biological sciences: including applications in statistics. John Wiley and Sons, New York, 296 pp.

Seemann, J., Yingst, A., Stuart-Smith, R. D., Edgar, G. J., and Altieri, A. H. (2018). The importance of sponges and mangroves in supporting fish communities on degraded coral reefs in Caribbean Panama. PeerJ, 6:e4455. https://doi.org/10.7717/peerj.4455 
Sella, I., and Benayahu, Y. (2010). Rearing cuttings of the soft coral Sarcophyton glaucum (Octocorallia, Alcyonacea): Towards mass production in a closed seawater system. Aquaculture Research, 41:1748-1758. https://doi.org/10.1111/j.1365-2109.2009.02475.x

Shaffer, M. R., Davy, S. K., and Bell, J. J. (2018). Hidden diversity in the genus Tethya: comparing molecular and morphological techniques for species identification. Heredity, 122:354-369. https://doi.org/10.1038/s41437-018-0134-6

Shenkar, N., Bronstein, O., and Loya, Y. (2008). Population dynamics of a coral reef ascidian in a deteriorating environment. Marine Ecology Progress Series, 367:163-171.

Shima, J. S., Osenberg, C. W., and St. Mary, C. M. (2008). Quantifying site quality in a heterogeneous landscape: Recruitment of a reef fish. Ecology, 89(1):86-94. https://doi.org/10.1890/07-0021.1

Silveira, C. B., Silva-Lima, A. W., Francini-Filho, R. B., Marques, J. S. M., Almeida, M. G., Thompson, C. C., Rezende, C. E., Paranhos, R., Moura, R. L., Salomon, P. S., and Thompson, F. L. (2015). Microbial and sponge loops modify fish production in phase-shifting coral reefs. Environmental Microbiology, 17(10):3832-3846. https://doi.org/10.1111/1462-2920.12851

Slattery, M., and Lesser, M. P. (2015). Trophic ecology of sponges from shallow to mesophotic depths (3 to $150 \mathrm{~m}$ ): Comment on Pawlik et al. (2015). Marine Ecology Progress Series, 527:275-279. https://doi.org/10.3354/meps11307

Smith, B., and Wilson, J. B. (1996). A consumer's guide to evenness indices. Oikos, 76(1):70-82.

Smith, J. E., Hunter, C. L., and Smith, C. M. (2010). The effects of top-down versus bottomup control on benthic coral reef community structure. Oecologia, 163:497-507.

Stearn, W., Scoffin, T. P., and Martindale, W. (1977). Calcium carbonate budget of a fringing reef on the west coast of Barbados. Bulletin of Marine Science, 27(3):479-510.

Steele, M. A. (1999). Effects of shelter and predators on reef fishes. Journal of Experimental Marine Biology and Ecology, 233:65-79. https://doi.org/10.1016/S0022-0981(98)00127-0

Stella, J. S., Pratchett, M. S., Hutchings, P. A., and Jones, G. P. (2011). Coral-associated invertebrates: diversity, ecological importance and vulnerability to disturbance. Oceanography and Marine Biology: An Annual Review, 49:43-104. 
Stevely, J. M., Sweat, D. E., Bert, T. M., Sim-Smith, C., and Kelly, M. (2011). Sponge mortality at Marathon and Long Key, Florida: Patterns of species response and population recovery. Proceedings of the Gulf and Caribbean Fisheries Institute, 63:384-400.

Stirling, G., and Wilsey, B. (2001). Empirical relationships between species richness, evenness, and proportional diversity. American Naturalist, 158(3):286-299.

Stubler, A. D., Furman, B. T., and Peterson, B. J. (2015). Sponge erosion under acidification and warming scenarios: Differential impacts on living and dead coral. Global Change Biology, 21:4006-4020. https://doi.org/10.1111/gcb.13002

Stubler, A. D., Furman, B. T., and Peterson, B. J. (2014). Effects of $p \mathrm{CO}_{2}$ on the interaction between an excavating sponge, Cliona varians, and a hermatypic coral, Porites furcata. Marine Biology, 161(8):1851-1859. https://doi.org/10.1007/s00227-014-2466-y

Suchanek, T. and Carpenter, R. (1983) Sponges as important space competitors in deep Caribbean coral reef communities. In: Contributions \#109 of the West Indies Laboratory (St. Croix, USVI) Symposium Series, 55-60.

Suding, K. N., Gross, K. L., and Houseman, G. R. (2004). Alternative states and positive feedbacks in restoration ecology. Trends in Ecology and Evolution, 19(1):46-53. https://doi.org/10.1016/j.tree.2003.10.005

Sutcliffe, P. R., Hooper, J. N. A., and Pitcher, C. R. (2010). The most common sponges on the Great Barrier Reef seabed, Australia, include species new to science (Phylum Porifera). Zootaxa, 2616:1-30. https://doi.org/ISSN 1175-5334

Tanaka, Y., Miyajima, T., Koike, I., Hayashibara, T., and Ogawa, H. (2008). Production of dissolved and particulate organic matter by the reef-building corals Porites cylindrica and Acropora pulchra. Bulletin of Marine Science, 82(2):237-245.

Tanner, J. E. (1995). Competition between scleractinian corals and macroalgae: An experimental investigation of coral growth, survival and reproduction. Journal of Experimental Marine Biology and Ecology, 190:151-168. https://doi.org/10.1016/S0196-0644(88)80272-5

Tanner, J. E., Hughes, T. P., and Connell, J. H. (1996). The role of history in community dynamics : A modelling approach. Ecology, 77(1):108-117. 
Taylor, M. W., Radax, R., Stegar, D., and Wagner, M. (2007). Sponge-associated microorganisms: evolution, ecology, and biotechnological potential. Microbiology and Molecular Biology Reviews, 71(2):295-347. https://doi.org/10.1128/MMBR.00040-06

Tebbett, S. B., Streit, R. P., and Bellwood, D. R. (2019). Expansion of a colonial ascidian following consecutive mass coral bleaching at Lizard Island, Australia. Marine Environmental Research, 144:125-129.

Teichberg, M., Wild, C., Bednarz, V. N., Kegler, H. F., Lukman, M., Gärdes, A. A., Heiden J. P., Weiand, L., Abu, N., Nasir, A., Miñarro, S., Ferse, S. C. A., Reuter, H., and PlassJohnson, J. G. (2018). Spatio-temporal patterns in coral reef communities of the Spermonde Archipelago, 2012-2014, I: Comprehensive reef monitoring of water and benthic indicators reflect changes in reef health. Frontiers in Marine Science, 5:33.

Tews, J., Brose, U., Grimm, V., Tielborger, K., Wichmann, M., Schwager, M., and Jeltsch, F. (2004). Animal species diversity driven by habitat heterogeneity/diversity: the importance of keystone structures. Journal of Biogeography, 31:79-92.

Thacker, R. W., Becerro, M. A., Lumbang, W. A., and Paul, V. J. (1998). Allelopathic interactions between sponges on a tropical reef. Ecology, 79(5):1740-1750.

Tibshirani, R. (1996). Regression shrinkage and selection via the Lasso. Journal of the Royal Statistical Society. Series B (Methodological), 58(1):267-288.

Topçu, N. E., Pérez, T., Grégori, G., and Harmelin-Vivien, M. (2010). In situ investigation of Spongia officinalis (Demospongiae) particle feeding: Coupling flow cytometry and stable isotope analysis. Journal of Experimental Marine Biology and Ecology, 389(1-2):61-69. https://doi.org/10.1016/j.jembe.2010.03.017

Trussell, G. C., Lesser, M. P., Patterson, M. R., and Genovese, S. J. (2006). Depth-specific differences in growth of the reef sponge Callyspongia vaginalis: Role of bottom-up effects. Marine Ecology Progress Series, 323:149-158. https://doi.org/10.3354/meps323149

Turchin, P., Lorio, P. L., Taylor, A. D., and Billings, R. F. (1991). Why do populations of southern pine beetles (Coleoptera, Scolytidae) fluctuate? Environmental Entomology, 20(2):401-409.

Turon, X., Becerro, M., Uriz, M., and Llopis, J. (1996). Small scale association measures in epibenthic communities as a clue for allelochemical interactions. Oecologia, 108(2):351-360. 
Vacelet, J., and Boury-Esnault, N. (1995). Carnivorous sponges. Nature, 373(6512):333335. https://doi.org/10.1038/373333a0

van Lier, J. R., Harasti, D., Laird, R., Noble, M. M., and Fulton, C. J. (2017). Importance of soft canopy structure for labrid fish communities in estuarine mesohabitats. Marine Biology, 164:45. https://doi.org/10.1007/s00227-017-3068-2

van Soest, R. W. M. (1989). The Indonesian sponge fauna: A status report. Netherlands Journal of Sea Research, 23:223-230.

van Soest, R. W. M., Boury-Esnault, N., Hooper, J. N. A., Rützler, K., de Voogd, N. J., Alvarez, B., Hajdu, E., Pisera, A. B., Manconi, R., Schönberg, C., Klautau, M., Picton, B., Kelly, M., Vacelet, J., Dohrmann, M., Díaz, M-C., Cárdenas, P., Carballo, J. L., Ríos, P., Downey, R. (2019). World Porifera Database. Accessed at http://www.marinespecies.org/porifera on 2019-08-02. doi:10.14284/359

van Soest, R. W. M., Boury-Esnault, N., Vacelet, J., Dohrmann, M., Erpenbeck, D., de Voogd, N. J., Santodomingo, N., Vanhoorne, B., Kelly, M., and Hooper, J. N. A. (2012). Global diversity of sponges (Porifera). PLoS ONE, 7(4):e35105. doi:10.1371/journal.pone.0035105

Vicente, J., Silbiger, N. J., Beckley, B. A., Raczkowski, C. W., and Hill, R. T. (2016). Impact of high $p \mathrm{CO}_{2}$ and warmer temperatures on the process of silica biomineralization in the sponge Mycale grandis. ICES Journal of Marine Science, 73(3):704-714

Vicente, V. P. (1990). Response of sponges with autotrophic endosymbionts during the coralbleaching episode in Puerto Rico. Coral Reefs, 8:199-202. https://doi.org/10.1007/BF00265011

Villamizar, E., Díaz, M. C., Rützler, K., and De Nóbrega, R. (2014). Biodiversity, ecological structure, and change in the sponge community of different geomorphological zones of the barrier fore reef at Carrie Bow Cay, Belize. Marine Ecology, 35:425-435. https://doi.org/10.1111/maec.12099

Wang, J.-T., Hsu, C.-M., Kuo, C.-Y., Meng, P.-J., Kao, S.-J., and Chen, C. A. (2015). Physiological outperformance at the morphologically-transformed edge of the cyanobacteriosponge Terpios hoshinota (Suberitidae: Hadromerida) when confronting opponent corals. PLoS One 10(6): e0131509. 
Wantiez, L., Chateau, O., and Le Mouellic, S. (2006). Initial and mid-term impacts of cyclone Erica on coral reef fish communities and habitat in the South Lagoon Marine Park of New Caledonia. Journal of the Marine Biological Association of the United Kingdom, 86:12291236.

Ward, J. H. (1963). Hierarchical grouping to optimize an objective function. Journal of the American Statistical Association, 58:236-244. https://doi.org/10.1080/01621459.1963.10500845

Ward-Paige, C. A., Risk, M. J., Sherwood, O. A., and Jaap, W. C. (2005). Clionid sponge surveys on the Florida Reef Tract suggest land-based nutrient inputs. Marine Pollution Bulletin, $51: 570-579$.

Warton, D. I., and Hui, F. K. C. (2011). The arcsine is asinine: the analysis of proportions in ecology. Ecology 92(1):3-10.

Webster, N. S. (2007). Sponge disease: A global threat? Environmental Microbiology, 9(6):1363-1375. https://doi.org/10.1111/j.1462-2920.2007.01303.x

Weijerman, M., Link, J. S., Fulton, E. A., Olsen, E., Townsend, H., Gaichas, S., Hansen, C., Skern-Mauritzen, M., Kaplan, I. C., Gamble, R., Fay, G., Savina, M., Ainsworth, C., van Putten, I., Gorton, R., Brainard, R., Larsen, K., and Hutton, T. (2016). Atlantis Ecosystem Model Summit: Report from a workshop. Ecological Modelling, 335:35-38. https://doi.org/10.1016/j.ecolmodel.2016.05.007

Weijerman, M., Fulton, E. A., Kaplan, I. C., Gorton, R., Leemans, R., Mooij, W. M., and Brainard, R. E. (2015). An integrated coral reef ecosystem model to support resource management under a changing climate. PLoS ONE, 10(12):e0144165. https://doi.org/10.1371/journal.pone.0144165

Weijerman, M., Kaplan, I., Fulton, E. A., Gorton, R., Grafeld, S., and Brainard, R. (2014). Design and parameterization of a coral reef ecosystem model for Guam. NOAA Technical Memorandum NMFS-PIFSC-43, (November). https://doi.org/10.7289/V5B27S76

Wenger, L. N., van Lier, J. R., and Fulton, C. J. (2018). Microhabitat selectivity shapes the seascape ecology of a carnivorous macroalgae-associated tropical fish. Marine Ecology Progress Series, 590:187-200. https://doi.org/10.3354/meps 12473 
Whalan, S., Abdul Wahab, M. A., Sprungala, S., Poole, A. J., and de Nys, R. (2015). Larval settlement: The role of surface topography for sessile coral reef invertebrates. PLoS ONE, 10(2):e0117675. https://doi.org/10.1371/journal.pone.0117675

Whalan, S., Webster, N. S., and Negri, A. P. (2012). Crustose coralline algae and a cnidarian neuropeptide trigger larval settlement in two coral reef sponges. PLoS ONE, 7(1):e30386. https://doi.org/10.1371/journal.pone.0030386

Whalan, S., Ettinger-Epstein, P., and de Nys, R. (2008). The effect of temperature on larval pre-settlement duration and metamorphosis for the sponge, Rhopaloeides odorabile. Coral Reefs, 27:783-786. https://doi.org/10.1007/s00338-008-0400-9

Wickham, H. (2009). ggplot2: Elegant Graphics for Data Analysis. New York: SpringerVerlag.

Wild, C., Hoegh-Guldberg, O., Naumann, M. S., Colombo-Pallotta, M. F., Ateweberhan, M., Fitt, W. K., Iglesias-Prieto, R., Palmer, C., Bythell, J. C., Ortiz, J.-C., Loya, Y., and van Woesik, R. (2011). Climate change impedes scleractinian corals as primary reef ecosystem engineers. Marine and Freshwater Research, 62:205-215. https://doi.org/10.1071/MF10254

Wild, C., Huettel, M., Klueter, A., Kremb, S. G., Rasheed, M. Y. M., and Jørgensen, B. B. (2004). Coral mucus functions as an energy carrier and particle trap in the reef ecosystem. Nature, 428:66-70. https://doi.org/10.1038/nature02333.1

Wilkinson, C R. (1987). Interocean differences in size and nutrition of coral reef sponge populations. Science, 236(4809):1654-1657. https://doi.org/10.1126/science.236.4809.1654

Wilkinson, C. R. (1983). Net primary productivity in coral reef sponges. Science, 219:410412.

Wilkinson, C. R., and Cheshire, A. C. (1990). Comparisons of sponge populations across the Barrier Reefs of Australia and Belize: evidence for higher productivity in the Caribbean. Marine Ecology Progress Series, 67:285-294. https://doi.org/10.3354/meps067285

Wilkinson, C. R., and Cheshire, A. C. (1989). Patterns in the distribution of sponge populations across the central Great Barrier Reef. Coral Reefs, 8:127-134.

Williams, C. B. (1964). Patterns in the balance of nature. London: Academic Press. 
Williams, I. D., Polunin, N. V. C., and Hendrick, V. J. (2001). Limits to grazing by herbivorous fishes and the impact of low coral cover on macroalgal abundance on a coral reef in Belize. Marine Ecology Progress Series, 222:187-196

Wilson, J. R., Ardiwijaya, R. L., and Prasetia, R. (2012). A study of the impact of the 2010 coral bleaching event on coral communities in Wakatobi National Park. The Nature Conservancy, Indo- Pacific Division, Indonesia. Report No. 7/12.

Wilson, S. K., Graham, N. A. J., Pratchett, M. S., Jones, G. P., and Polunin, N. V. C. (2006). Multiple disturbances and the global degradation of coral reefs: Are reef fishes at risk or resilient? Global Change Biology, 12:2220-2234. https://doi.org/10.1111/j.13652486.2006.01252.x

Wisshak, M., Schönberg, C. H. L., Form, A., and Freiwald, A. (2014). Sponge bioerosion accelerated by ocean acidification across species and latitudes? Helgoland Marine Research, 68(2):253-262. https://doi.org/10.1007/s10152-014-0385-4

Wisshak, M., Schönberg, C. H. L., Form, A., and Freiwald, A. (2013). Effects of ocean acidification and global warming on reef bioerosion-lessons from a clionaid sponge. Aquatic Biology, 19:111-127. https://doi.org/10.3354/ab00527

Wisshak, M., Schoenberg, C. H. L., Form, A., and Freiwald, A. (2012). Ocean acidification $\begin{array}{lllll}\text { accelerates } & \text { reef } & \text { bLoerosion. } & \text { ONE, } & \text { (9)45124. }\end{array}$ https://doi:10.1371/journal.pone.0045124

Wood, R. (1995). The changing biology of reef-building. PALAIOS, 10(6):517-529.

Wooster, M. K., McMurray, S. E., Pawlik, J. R., Morán, X. A. G., and Berumen, M. L. (2019). Feeding and respiration by giant barrel sponges across a gradient of food abundance in the Red Sea. Limnology and Oceanography, 64:1790-1801. https://doi.org/10.1002/lno.11151

Wörheide, G., Solé-Cava, A. M., and Hooper, J. N. a. (2005). Biodiversity, molecular ecology and phylogeography of marine sponges: patterns, implications and outlooks. Integrative and Comparative Biology, 45:377-385. https://doi.org/10.1093/icb/45.2.377

Wright, D. H. (1983). Species-energy theory: An extension of species-area theory. Oikos, 41(3):496-506. 
Wulff, J. L. (2017). Bottom-up and top-down controls on coral reef sponges: disentangling within habitat and between-habitat processes. Ecology, 98(4):1130-1139. https://doi.org/10.1002/ecy.1754

Wulff, J. L. (2013). Recovery of sponges after extreme mortality events: Morphological and taxonomic patterns in regeneration versus recruitment. Integrative and Comparative Biology, 53(3):512-523.

Wulff, J. L. (2006a). Ecological interactions of marine sponges. Canadian Journal of Zoology, 84:146-166. https://doi.org/10.1139/z06-019

Wulff, J. L. (2006b). Rapid diversity and abundance decline in a Caribbean coral reef sponge community. Biological Conservation, 127:167-176.

Wulff, J. L. (2001). Assessing and monitoring coral reef sponges: why and how? Bulletin of Marine Science, 69(2):831-846.

Wulff, J. L. (1997). Parrotfish predation on cryptic sponges of Caribbean coral reefs. Marine Biology, 129:41-52. https://doi.org/10.1007/s002270050144

Wulff, J. L. (1984). Sponge-mediated coral reef growth and rejuvenation. Coral Reefs, 3:157-163.

Wulff, J. L., and Buss, L. W. (1979). Do sponges help hold coral reefs together? Nature, 281:474-475.

Yahel, G., Sharp, J. H., Marie, D., Häse, C., and Genin, A. (2003). In situ feeding and element removal in the symbiont-bearing sponge Theonella swinhoei: Bulk DOC is the major source for carbon. Limnology and Oceanography, 48(1):141-149. https://doi.org/10.4319/lo.2003.48.1.0141

Yodzis, P. (1988). The indeterminacy of ecological interactions as perceived through perturbation experiments. Ecology, 69:508-515.

Young, G. C., Dey, S., Rogers, A. D., and Exton, D. (2017). Cost and time-effective method for multiscale measures of rugosity, fractal dimension, and vector dispersion from coral reef 3D models. PLoS ONE, 12(4):e0175341. https://doi.org/10.1371/journal.pone.0175341

Zawada, K. J. A., Madin, J. S., Baird, A. H., Bridge, T. C. L., and Dornelas, M. (2019). Morphological traits can track coral reef responses to the Anthropocene. Functional Ecology, 33:962-975. https://doi.org/10.1111/1365-2435.13358 
Zea, S. (1993). Cover of sponges and other sessile organisms in rocky and coral reef habitats of Santa Marta, Colombian Caribbean Sea. Caribbean Journal of Science, 29(1-2):75-88.

Zhang, F., Blasiak, L. C., Karolin, J. O., Powell, R. J., Geddes, C. D., and Hill, R. T. (2015). Phosphorus sequestration in the form of polyphosphate by microbial symbionts in marine sponges. Proceedings of the National Academy of Sciences, 112(14):4381-4386. https://doi.org/10.1073/pnas.1423768112

Zhang, F., Vicente, J., and Hill, R. T. (2014). Temporal changes in the diazotrophic bacterial communities associated with Caribbean sponges Ircinia stroblina and Mycale laxissima. Frontiers in Microbiology, 5:561. https://doi.org/10.3389/fmicb.2014.00561

Zhang, H., John, R., Peng, Z., Yuan, J., Chu, C., Du, G., and Zhou, S. (2012). The relationship between species richness and evenness in plant communities along a successional gradient: a study from sub-alpine meadows of the eastern Qinghai-Tibetan Plateau, China. PLoS ONE, 7(11):e49024. https://doi.org/10.1371/journal.pone.0049024

Zocchi, E., Basile, G., Cerrano, C., Bavestrello, G., Giovine, M., Bruzzone, S., Guida, L., Carpaneto, A., Magrassi, R., and Usai, C. (2003). ABA- and cADPR-mediated effects on respiration and filtration downstream of the temperature-signaling cascade in sponges. Journal of Cell Science, 116:629-636. https://doi.org/10.1242/jcs.00277

Zou, H., and Hastie, T. (2005). Regularization and variable selection via the elastic net. Journal of the Royal Statistical Society. Series B: Statistical Methodology, 67(5):768. https://doi.org/10.1111/j.1467-9868.2005.00527.x 


\section{Appendix 1. Overview of the qualitative modelling approach}

A brief overview of the qualitative modelling of complex systems is provided here. The first stage of the qualitative approach is the representation of the chosen system. This step requires careful evaluation of the components and the processes that must be captured, and the exclusion of those that are not central to the investigation or are deemed to be relatively less important in the specific context. This process inevitably leads to the exclusion of some ecological processes, with the risk of oversimplifying the system. One solution to this problem, aided by the economy of qualitative models, is to design and evaluate a set of models incorporating different assumptions, in an attempt to reach a compromise between the complexity of the real world and the tractability of its representation.

When representing the system, three equivalent notations can be used to summarise the relationships between its variables. First, the interactions between two variables can be represented as Lotka-Volterra equations, which detail the contribution of one variable to the birth and death rate of the other, and vice versa. For a simple predator-prey system of a variable $\mathrm{N}_{2}$ feeding on another variable $\mathrm{N}_{1}$, this can be written as:

$$
\begin{aligned}
& \frac{d N_{1}}{d t}=N_{1}\left(\beta-\alpha_{1,2} N_{2}\right) \\
& \frac{d N_{2}}{d t}=N_{2}\left(\alpha_{2,1} N_{1}-\delta\right)
\end{aligned}
$$

where $\beta$ and $\delta$ are a birth rate and a death rate, respectively, and $\alpha_{\mathrm{i}, \mathrm{j}}$ defines the relationship between $\mathrm{N}_{\mathrm{i}}$ and $\mathrm{N}_{\mathrm{j}}$, and more specifically the effect of $\mathrm{N}_{\mathrm{j}}$ on $\mathrm{N}_{\mathrm{i}}$. As from Dambacher et al. (2002), for a system at or near equilibrium this can be generalised as:

$$
d N_{i}^{*} / d t=f_{i}\left(N_{1}, N_{2}, \ldots, N_{n} ; C_{1}, C_{1}, \ldots, C_{h}\right)
$$

where the change in the equilibrium population $N_{i}$ in a system counting $n$ populations is a function of all other populations and the parameters that describe their growth and their interactions with $N_{i}$.

Second, the direct relationships between the variables are detailed in the community or Jacobian matrix A. For a system composed of three populations, for example: 


$$
\boldsymbol{A}=\left[\begin{array}{lll}
a_{1,1} & a_{1,2} & a_{1,3} \\
a_{2,1} & a_{2,2} & a_{2,3} \\
a_{3,1} & a_{3,2} & a_{3,3}
\end{array}\right]
$$

This is to be read vertically, i.e. $a_{i j}$ details the effects of $j$ on $i$. Elements along the matrix diagonal indicate self-effects. The sign structure of this matrix describes the type of link between two variables. A (-) sign indicates a negative effect of variable $j$ on variable $i,(+)$ a positive effect, and (0) no effect. Formally, the community matrix describes the relationship between population abundance $\mathbf{N}$ and the vector $\mathbf{K}$ of the carrying capacity of the system, such that $\mathbf{A N}=\mathbf{K}$ (Levins 1968). The community matrix and its adjoint and inverse are powerful tools for exploring the structure of a community and the effects of a perturbation acting on one of its resource variables or species, which is the approach we have taken.

The third alternative notation is the signed digraph, where variables are represented by circles and the interactions between them by lines (Fig. A1.1). The ends of the lines indicate the effect of the source variable on the target variable, with $(\rightarrow)$ denoting a positive effect, $(--\bullet)$ a negative effect, and (--) no effect. Combining these three functions, it is possible to represent all biological interactions occurring in nature between two populations, such as a predator-prey interaction $(\bullet \rightarrow)$ and competition $(\bullet-\bullet)$. Arrows pointing to their source variable represent self-effects and are usually negative, representing either a density-dependent mechanism (stemming from limited resources such as space or food), or the reliance of a population upon food sources not represented in the model. The relationships between variables are assumed to be linear for simplicity, although more complex functions could be specified where there is sufficient information to characterise them.

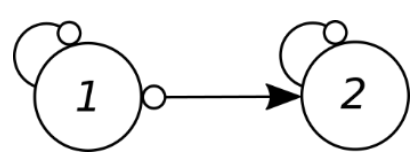

Figure A1.1. Signed digraph of a predator (2) feeding on a prey item (1). Both variables are self-dampened.

\section{System stability}

After defining and representing the system, an evaluation of its stability must be performed. The mathematical, or "Lyapunov" stability of a system is described as its ability to "persist in spite of perturbations" (Logofet 1993). More generally, a system is considered stable if it is able to return to its original state after being exposed to a sudden and small perturbation (Dambacher et al. 2003b). The qualitative approach starts from the assumption that the 
observed system is stable; that means at or close to equilibrium. In order to understand the concept of local stability, some definition of feedback must be provided. For a system with $n$ variables there are $n$ levels of feedback $\left(F_{k}\right)$, and each level has length $k$. Each $F_{k}$ is defined as the summation of the closed loops of length $k$ and the product of the disjoint loops, i.e. those that do not share variables with the direct loop. For example, a system with $n=3$ variables will have feedback levels $F_{1}, F_{2}$ and $F_{3} . F_{1}$ is the summation of all feedbacks of length $k=1$, whereas $\mathrm{F}_{2}$ is the summation of all feedbacks of length $k=2$ and all the products of disjoint loops of length $k=1 . \mathrm{F}_{3}$ follows the same logic as $\mathrm{F}_{2}$; it is called system feedback because it is the feedback at highest level (here $n=3$ ), and it coincides with the system determinant (derived from the community matrix). The reader is directed to Levins (1974) for a comprehensive discussion of the topic. Based on this definition of feedback, two criteria to assess the stability (or lack thereof) of a system can be formally described. These criteria, specified by Dambacher et al. (2003b), derive from Hurwitz's principal theorem, and are both necessary and sufficient conditions for Lyapunov stability.

There are two main potential sources of instability in a qualitative model. The first is the predominance of positive feedback in the system. Positive feedbacks are not uncommon in natural systems, but if they are not counterbalanced by stronger complementary negative feedbacks they can lead to a self-amplification of the response to a perturbation, which will cause the system to drift away from equilibrium in the direction of the original displacement (Levins 1974). Therefore, for local stability to be attained, negative feedbacks must always prevail over positive feedbacks at all levels, i.e. the net feedback $F_{k}$ must always be negative for all $k=1: n$. This first criterion of stability is called Hurwitz criterion i (Dambacher et al. 2003b).

The second source of instability is the presence of strong negative feedback at higher levels in the system, i.e. long loops have stronger negative feedback than the product of low-order disjoint loops. This condition causes the system to have unstable oscillations, because feedback loops at low levels are not strong enough to keep the system close to its original state when perturbed. Thus, negative feedback must become weaker while the length of the loops increases, i.e. $F_{1} F_{2}+F_{3}<0$ for the above introduced 3-variable example. This is Hurwitz stability criterion ii, and it depends on feedback at lower levels of the system being strong enough to counterbalance feedback at higher levels. 
Positive feedbacks are always present in the system although they may not be dominating, especially when the represented system includes many links and variables in an attempt to keep a satisfactory level of realism. Therefore, it is often safer to refer to conditional stability, that is, the system is stable provided that negative loops are stronger than the positive loops. Because qualitative modelling does not attempt to quantify, or weight, the intensity of each interaction, it is potentially impossible to assess whether this condition will be satisfied, and thus it is wise to identify the potential source of instability. On the basis of Hurwitz criteria $\mathrm{i}$ and ii, a model can be classified as a class I or class II model. For the detailed rationale behind this classification see Dambacher et al. (2003b). If the model does not violate criterion ii, then it is defined as a class I model. Class I models are prone to instability due to failure of Hurwitz criterion i. In other words, strong enough positive feedbacks may overcome negative feedbacks, although the net feedback is $F_{k}<0$ for all $k=1: n$. Models not failing criterion i may still fail criterion ii, and belong to class II models. These models are prone to instability due to the presence of stronger feedback at higher levels. A class I model may still be unstable if positive feedbacks overwhelm negative ones, but it satisfies both Hurwitz's criteria and is therefore deemed as stable.

\section{Analyses of perturbations}

After evaluation of model stability, the focus can move to the exploration of how the effects of a press perturbation on the system propagate. A press perturbation is defined as an event that causes either a positive or a negative change in the demographic parameters (e.g. birth rate and death rate) of one or several model components, and lasts long enough to drive the system towards a new equilibrium point. For example, a positive press perturbation acting on one population causes either an increase in its birth rate or a decrease in its death rate. Alternatively, a negative perturbation will result in a decrease in the birth rate or an increase in the death rate. This change in the demographic rates of one model component will have consequences for the rest of the system that depend on the links between the altered variable and the others. Model components or resource variables directly linked to the altered ones will experience direct effects; for instance the decline of a predator may result in the increase of its prey, or vice versa. However, for more complex systems, non-trivial dynamics emerge, and complex feedback loops may cause unexpected responses of some variables to the perturbation. One of the goals of the qualitative approach is to predict the qualitative response of all variables in the system following a press perturbation. 
The impact of a perturbation on a system can be derived with Levin's algorithm (Levins 1974), or with the use of the adjoint matrix. Levin's algorithm defines the response of a variable $j$ to a perturbation acting on the set of parameters $\mathrm{C}$ of a variable $i$ as:

$$
\frac{d N_{j}^{*}}{d C_{h}}=\frac{\sum_{i, k} \frac{d f_{i}}{d C_{h}} p_{j i}^{(k)} F_{n-k}^{(p-)}}{F_{n}}
$$

This means that the response is a function of the direct effect of the perturbation on the set of parameters $C$, the direct paths $p$ from $i$ to $j$ of all possible lengths $k$, the complementary feedback $F^{(p-)}$ for each direct path, and the system feedback $F_{n}$. These elements define how the perturbation propagates through the modelled system, depending on the interactions between variables. As a result, the response is given by the summation of a potentially large number of positive and negative feedbacks that influence how a variable reacts to the perturbation.

The effects of a perturbation can also be determined with the use of the adjoint matrix. Given the community matrix $\mathbf{A}$, the adjoint matrix $\operatorname{adj}(\mathbf{A})$ is defined as the transpose of the matrix of the cofactors (Searle 1966), and it is related to the inverse $\mathbf{A}^{-1}$ by the algebraic equality:

$$
\boldsymbol{A}^{-1}=\frac{\operatorname{adj}(\boldsymbol{A})}{\operatorname{det} \boldsymbol{A}}
$$

The adjoint matrix details the relative strength of complementary feedbacks, or in other words the net feedback from the perturbed variable to the response variable. Therefore, the sign of the elements $a_{i j}$ of the adjoint indicates whether a variable $i$ is affected positively, negatively or is not affected at all by the perturbation of variable $j$. The adjoint matrix presents the advantage of not requiring the weighting or quantification of the interactions between the system variables, as the inverse matrix does. Combined effects of the perturbation of multiple variables are calculated by adding the columns corresponding to all perturbed variables in the adjoint and absolute feedback (T) matrices and using the sums to compute prediction weights for the combinatory effect (see Dambacher et al. 2002).

\section{$\underline{\text { Sign indeterminacy and ambiguity of the predictions }}$}

The drawback of the use of the adjoint matrix is that each of its elements is the summation of a potentially very large number of feedback loops of opposite sign, intuitively in the same way as the response computed with Levins' algorithm. Therefore, the net feedback that is detailed by each matrix element can suffer from high sign indeterminacy; that is, the ambiguity of the prediction of the sign of the net feedback. The net feedback is subject to the strength of 
the single feedbacks, but because the single interactions are not quantified, it is not possible to assess whether the actual sign of the response in the real system will be coherent with the sign predicted by the adjoint matrix. Dambacher et al. (2003a) proposed a procedure to estimate the likelihood of each element of the adjoint matrix to successfully predict the sign of the response to a perturbation. This protocol is based on the ratio between net and absolute number of feedbacks for each element of the adjoint matrix. Specifically, the element-wise division of each element of the adjoint matrix by each element of the absolute feedback matrix $\mathbf{T}$ yields a matrix of weights between 0 and 1, denoted as W. Numeric simulations from Dambacher et al. (2003a) demonstrated that a value of $\mathrm{W} \geq 0.5$ corresponds to a $>90 \%$ chance of an accurate sign prediction in the adjoint matrix, which is the threshold the authors recommend, and it was chosen in this work as discriminant between ambiguous and unambiguous predictions.

It is noteworthy that sign indeterminacy is a function of the number of complementary feedbacks, which increase factorially with the number of variables in the model. Therefore, complexity and realism in the representation of the system come at the cost of ambiguity of the predictions. Because a high-realism model yielding completely inconclusive predictions is of little value, this limitation is an incentive to contain the model to the elements that really are central to the investigation, and to sacrifice any unnecessary detail.

Signed digraphs were developed with the software PowerPlay Digraph Editor, Version 2.0 (2002). Stability and perturbation analysis were carried out with the mathematical software available at Supplement 1 of Dambacher et al. (2002) in Ecological Archives E083-022-S1 at http://www.esapubs.org/archive/. All models were sign-stable and satisfied both Hurwitz's criteria i and ii, being classified as class I models. 


\section{Appendix 2. Temporal variability of sponge abundance and biodiversity of an Indonesian sponge assemblage}

Table A2.1. List of the 42 taxonomically identified sponge taxa of the Hoga sponge assemblage. The remaining taxa were not possible to identify, largely due to lack of suitable samples or as a result of not being able to export specimens from Indonesia in many years of the survey.

\begin{tabular}{|c|c|c|}
\hline Species & Family & Class \\
\hline Aaptos sp. & Suberitidae & Demospongiae \\
\hline Acanthella sp. 1 & Dictyonellidae & Demospongiae \\
\hline Acanthella sp. 2 & Dictyonellidae & Demospongiae \\
\hline Agelas sp. & Agelasidae & Demospongiae \\
\hline Axinella sp. & Axinellidae & Demospongiae \\
\hline Axinyssa sp. & Halichondriidae & Demospongiae \\
\hline Chalinula milnei & Chalinidae & Demospongiae \\
\hline Chalinula nematifera & Chalinidae & Demospongiae \\
\hline Chalinula sp. & Chalinidae & Demospongiae \\
\hline Chelonaplysilla sp. 1 & Darwinellidae & Demospongiae \\
\hline Chelonaplysilla sp. 2 & Darwinellidae & Demospongiae \\
\hline Cinachyrella sp. 1 & Tetillidae & Demospongiae \\
\hline Cinachyrella sp. 2 & Tetillidae & Demospongiae \\
\hline Cinachyrella sp. 3 & Tetillidae & Demospongiae \\
\hline Clathria (Microciona) mima & Microcionidae & Demospongiae \\
\hline Clathrina sp. 1 & Clathrinidae & Calcarea \\
\hline Clathrina sp. 2 & Clathrinidae & Calcarea \\
\hline Clathrina sp. 3 & Clathrinidae & Calcarea \\
\hline Clathrina sp. 4 & Clathrinidae & Calcarea \\
\hline Clathrina sp. 5 & Clathrinidae & Calcarea \\
\hline Clathrina sp. 6 & Clathrinidae & Calcarea \\
\hline
\end{tabular}




\begin{tabular}{|c|c|c|}
\hline Species & Family & Class \\
\hline Cliona sp. & Clionaidae & Demospongiae \\
\hline Cribrochalina sp. & Niphatidae & Demospongiae \\
\hline Dendrilla sp. & Darwinellidae & Demospongiae \\
\hline Gelliodes sp. & Niphatidae & Demospongiae \\
\hline Haliclona sp. & Chalinidae & Demospongiae \\
\hline Jaspis splendens & Ancorinidae & Demospongiae \\
\hline Lamellodysidea herbacea & Dysideidae & Demospongiae \\
\hline Leucetta sp. & Leucettidae & Calcarea \\
\hline Liosina sp. & Dictyonellidae & Demospongiae \\
\hline Monanchora unguiculata & Crambeidae & Demospongiae \\
\hline Niphates sp. 1 & Niphatidae & Demospongiae \\
\hline Niphates sp. 2 & Niphatidae & Demospongiae \\
\hline Niphates sp. 3 & Niphatidae & Demospongiae \\
\hline Niphates sp. 4 & Niphatidae & Demospongiae \\
\hline Pericharax sp. & Leucettidae & Calcarea \\
\hline Placospongia melobesioides & Placospongiidae & Demospongiae \\
\hline Protosuberites sp. & Suberitidae & Demospongiae \\
\hline Pseudoceratina sp. 1 & Pseudoceratinidae & Demospongiae \\
\hline Pseudoceratina sp. 2 & Pseudoceratinidae & Demospongiae \\
\hline Sycon sp. & Sycettidae & Calcarea \\
\hline Xestospongia sp. & Petrosiidae & Demospongiae \\
\hline
\end{tabular}



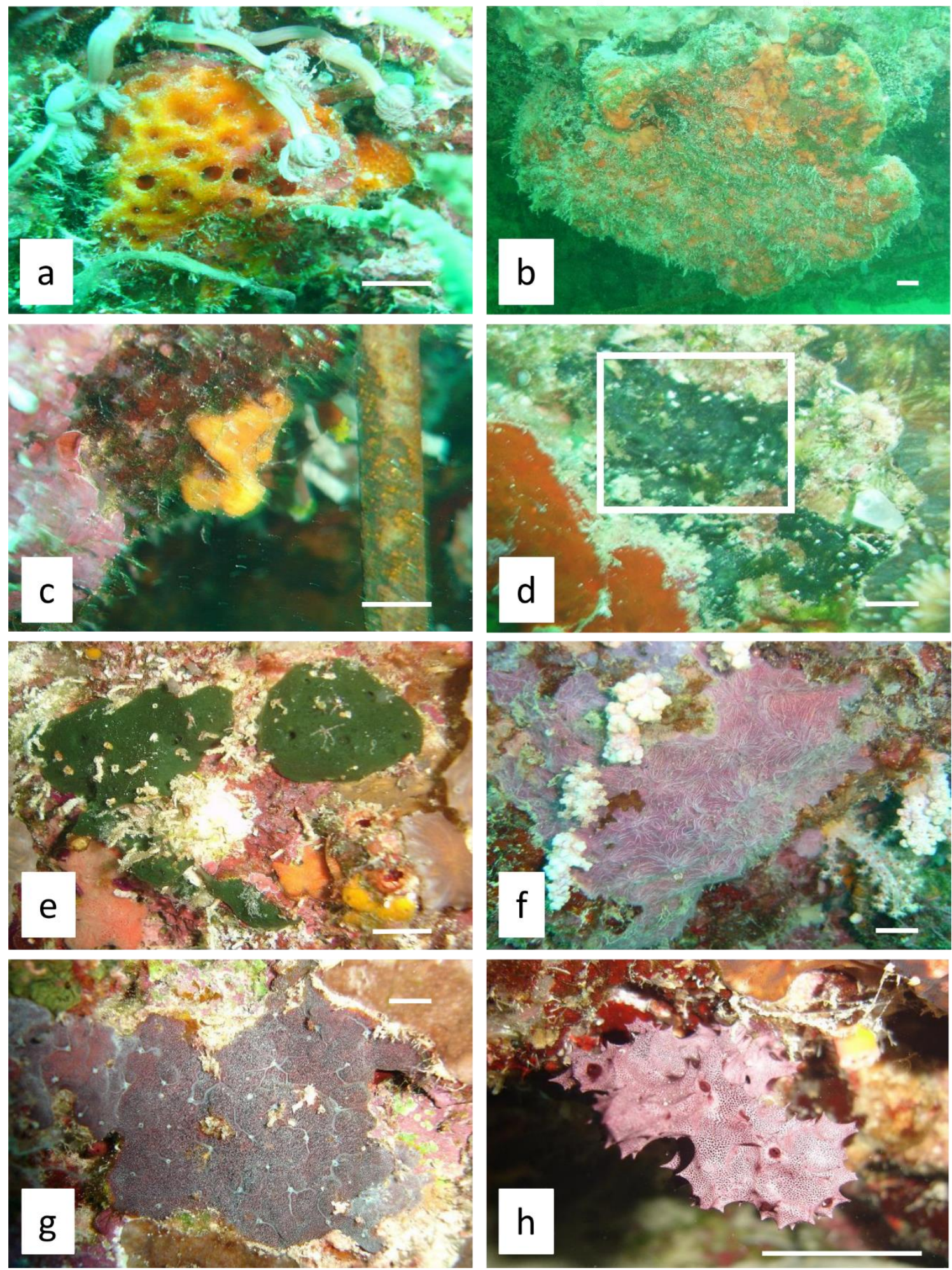

Figure A2.1. Some common sponge taxa from the Hoga Island assemblage. Bars correspond to $1 \mathrm{~cm}$. A: Acanthella sp.; b: Agelas sp.; c: Axinella sp.; d: Axinyssa sp.; e: Chalinula milnei; f: Chalinula nematifera; g: Chalinula sp.; h: Chelonaplysilla sp. 1. 

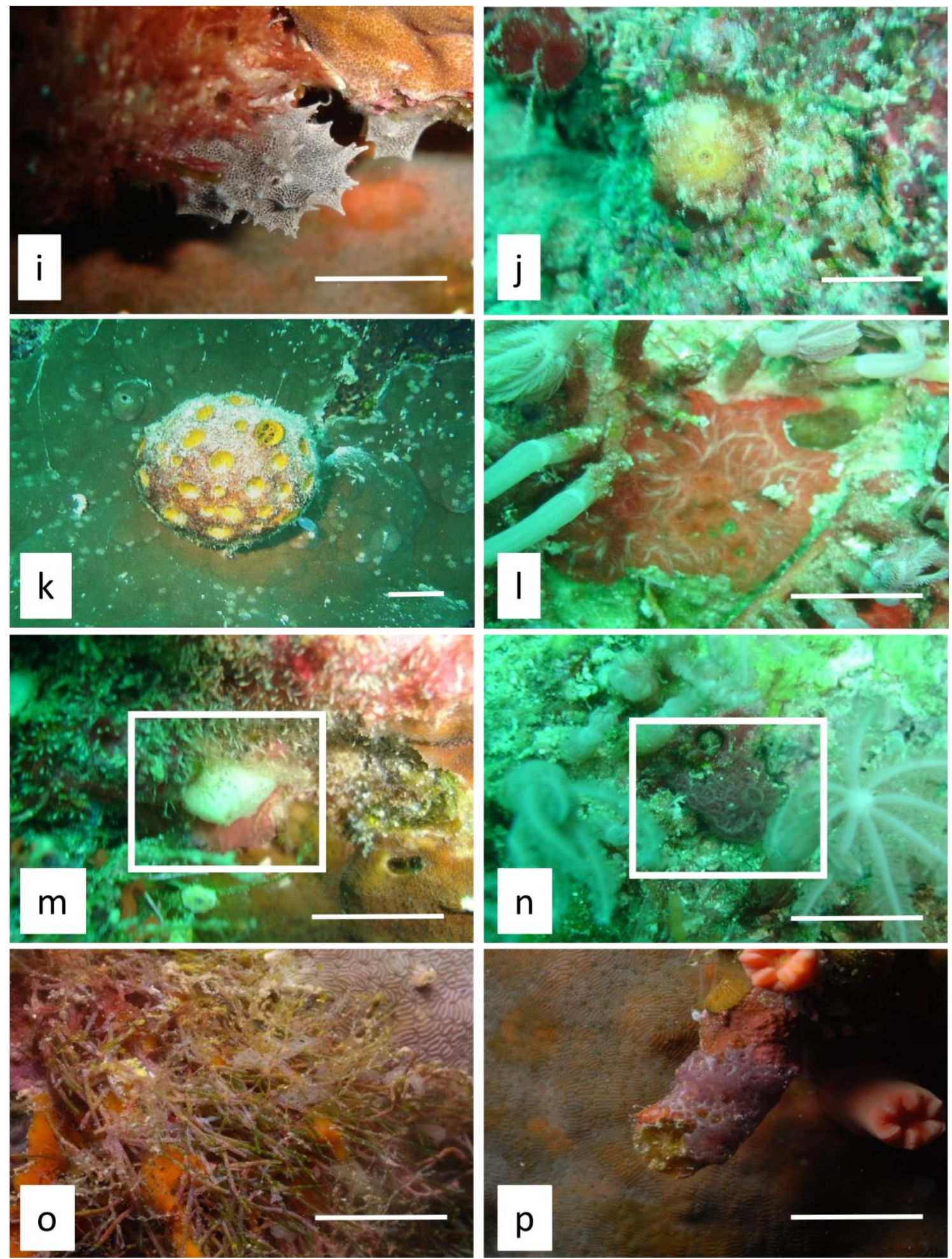

Figure A2.1: Continued. I: Chelonaplysilla sp. 2; j: Cinachyrella sp. 2; k: Cinachyrella sp. 3; 1: Clathria (Microciona) mima; m: Clathrina sp. 1; n: Clathrina sp. 2; o: Clathrina sp. 3 (white in association with turf algae); p: Clathrina sp. 4. 

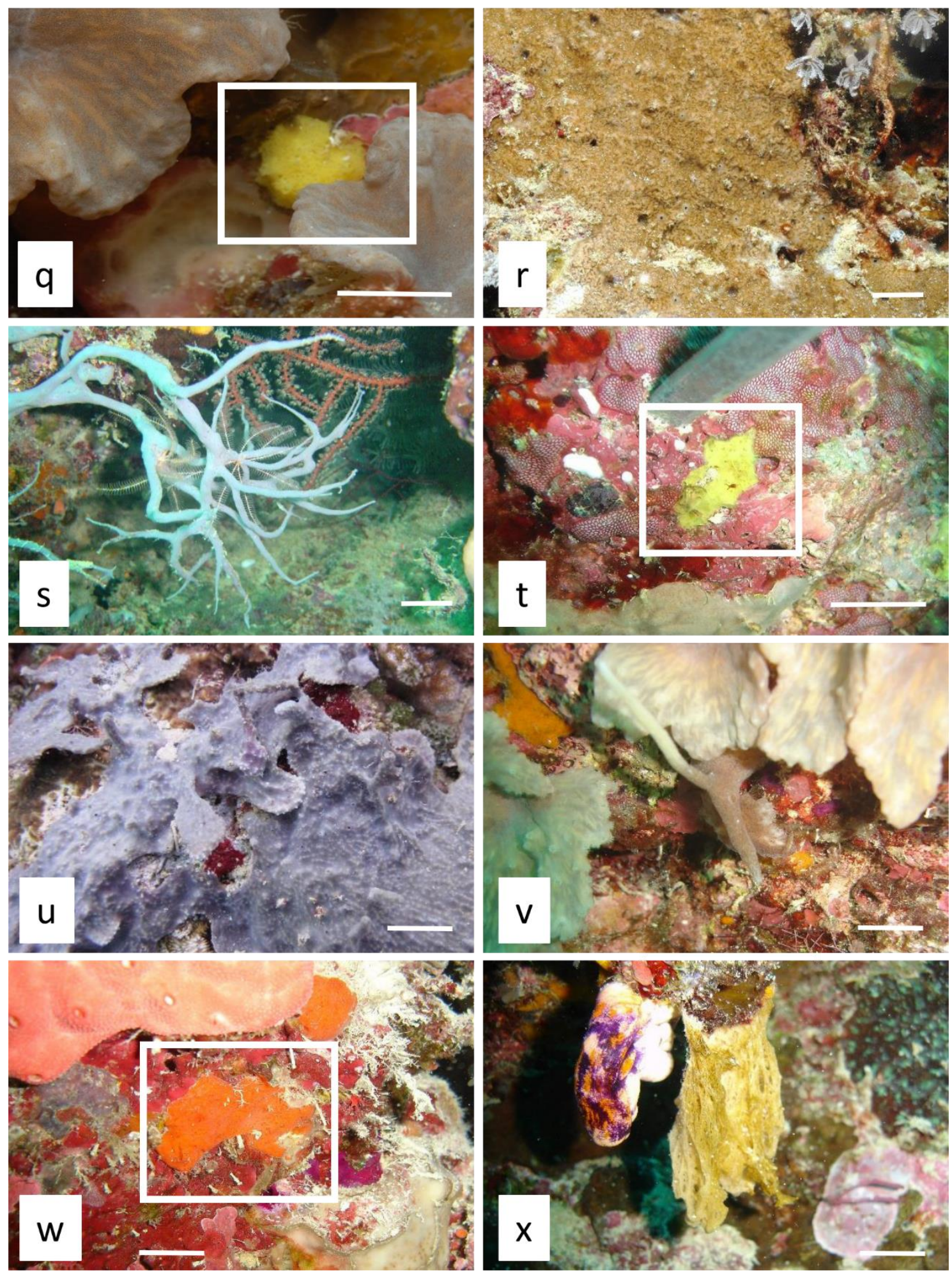

Figure A2.1: Continued. Q: Clathrina sp. 6; r: Cliona sp.; s: Cribrochalina sp.; t: Dendrilla sp.; u: Lamellodysidea herbacea; v: Haliclona sp.; w: Jaspis splendens; x: Leucetta sp. 

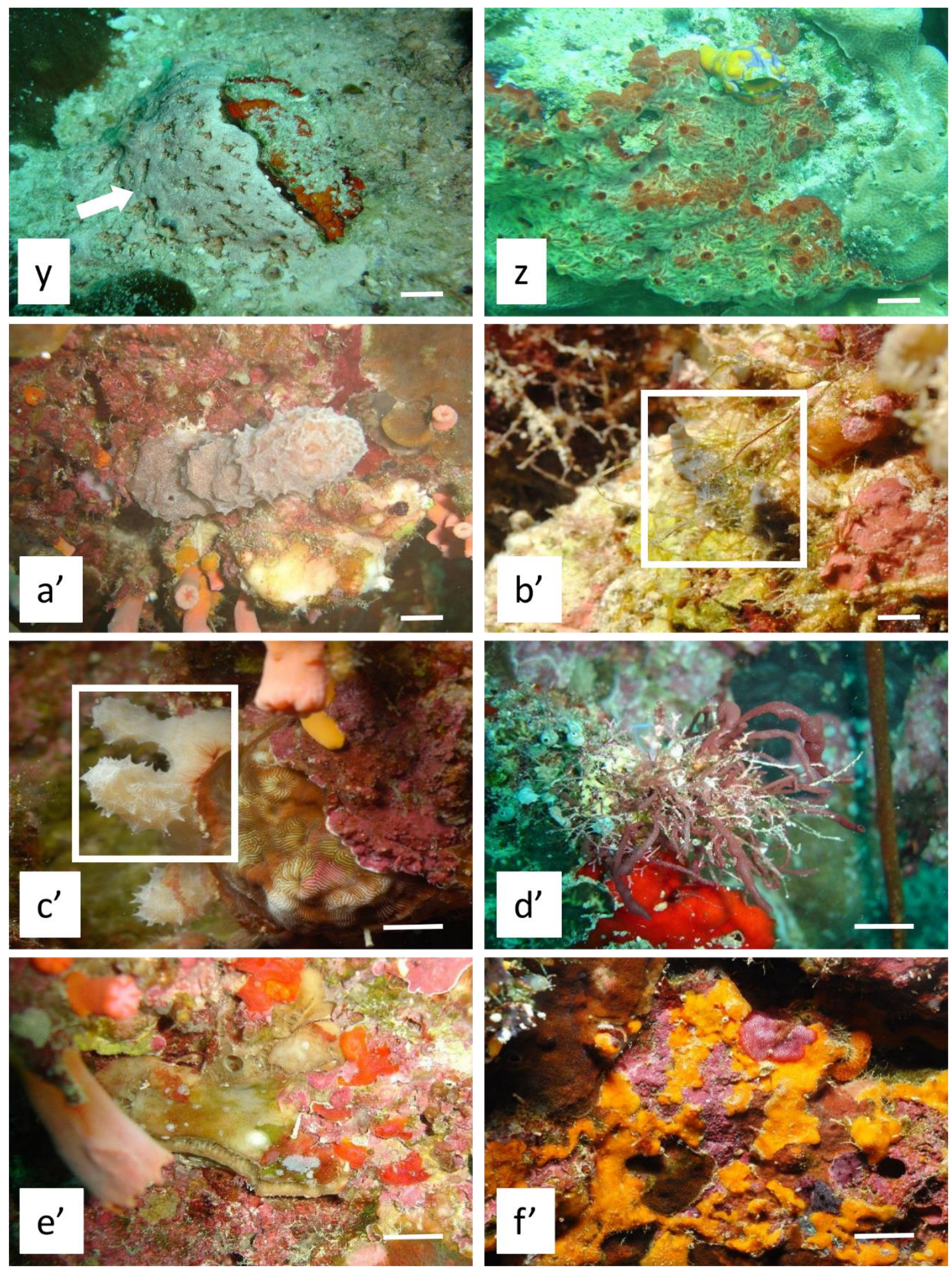

Figure A2.1: Continued. Y: Liosina sp.; z: Monanchora unguiculata; a' Niphates sp. 1; b':

Niphates sp. 2; c': Niphates sp. 3; d': Pericharax sp.; e': Placospongia melobesioides; f':

Protosuberites sp. 

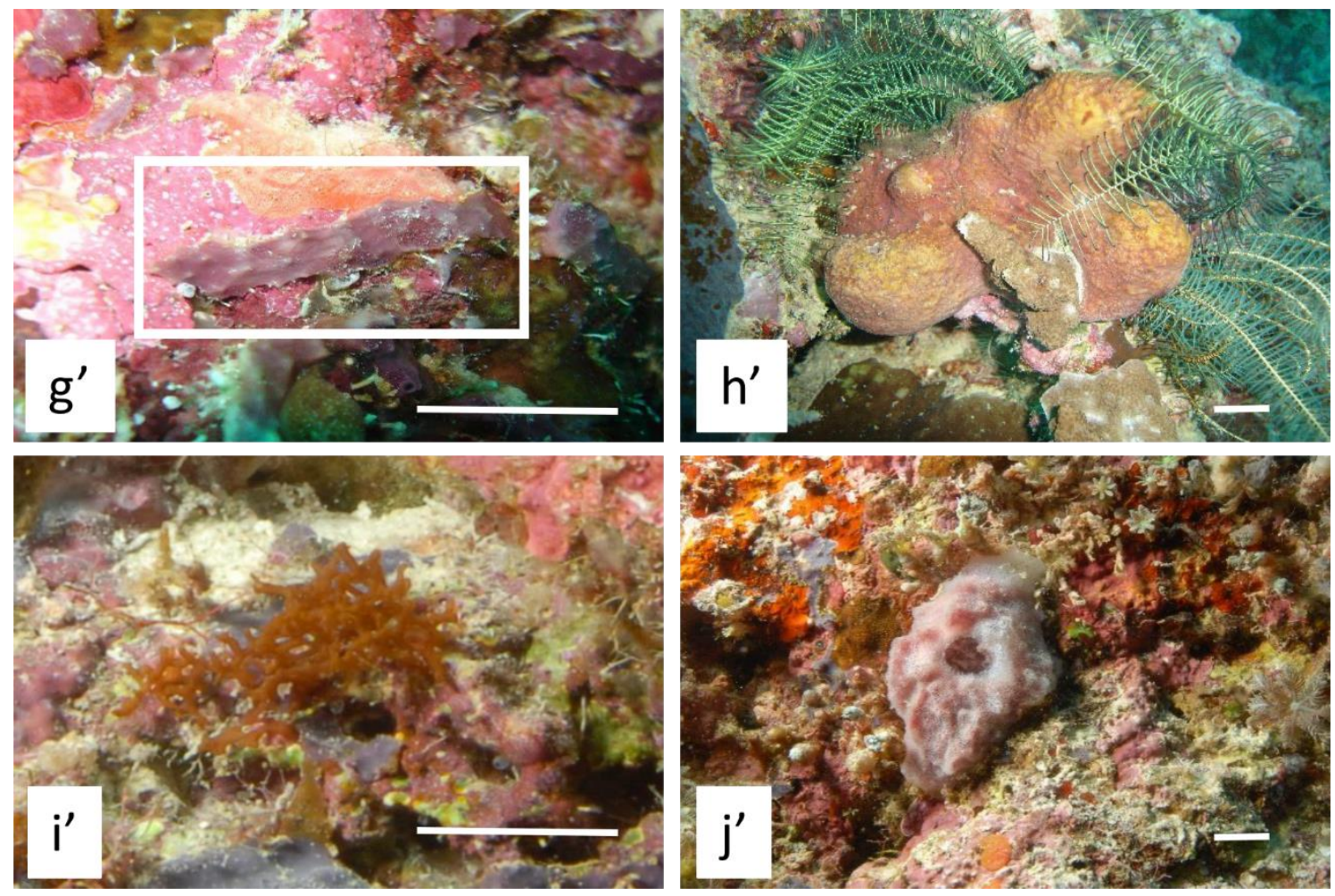

Figure A2.1: Continued. G': Pseudoceratina sp. 1; h': Pseudoceratina sp. 2; i’: Sycon sp.; j': Xestospongia sp. 
a

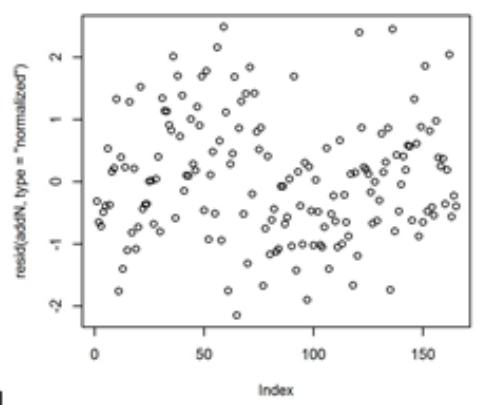

b

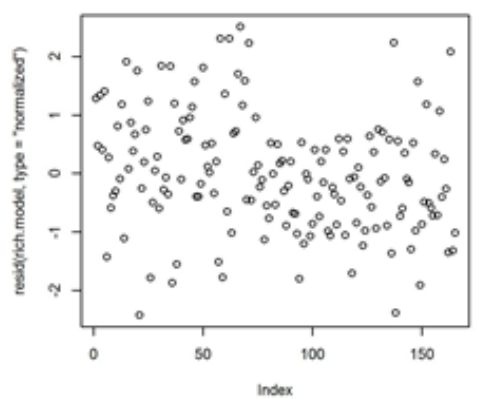

C
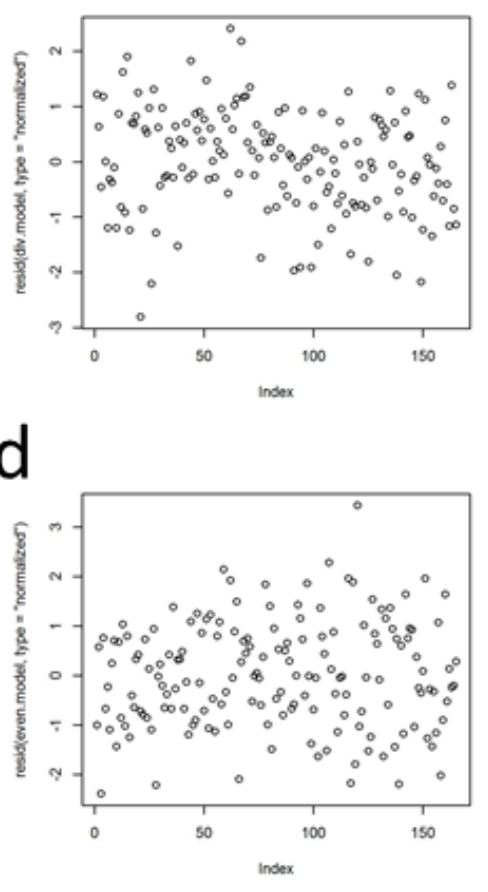

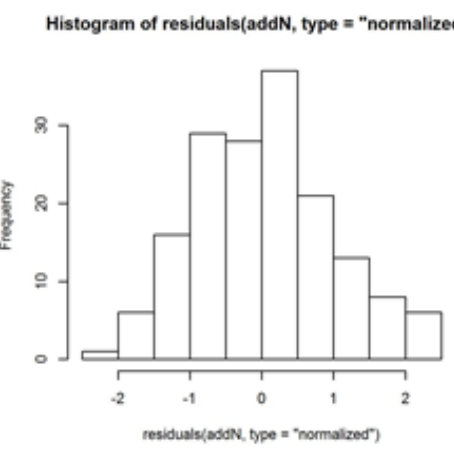

Histogram of residuals(rich.model, type = "normalized"

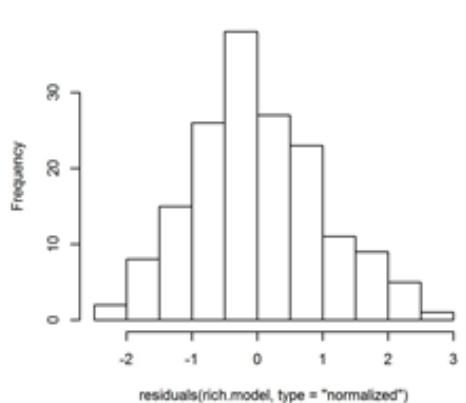

Histogram of residuals(div.model, type = "normalized"

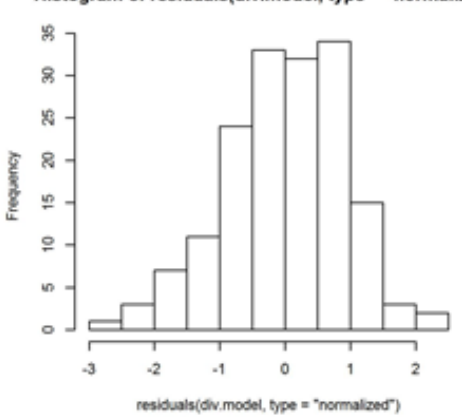

Histogram of residuals(even-model, type = "normalized"

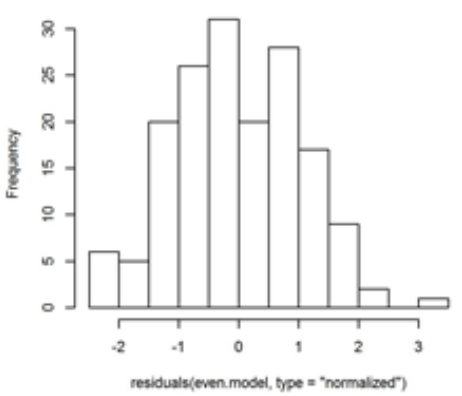

Normal Q-Q Plot

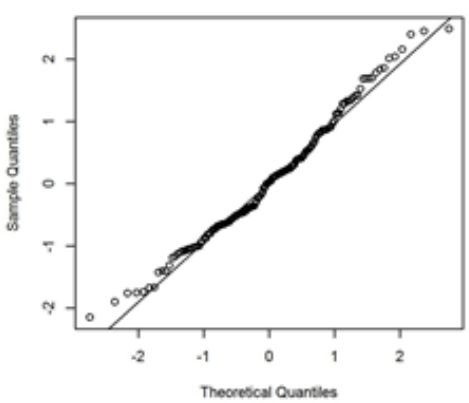

Normal Q-Q Plot

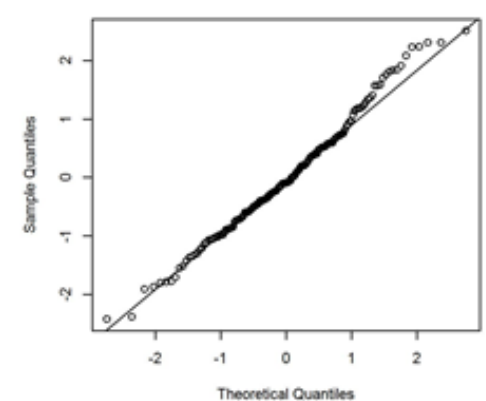

Normal Q-Q Plot

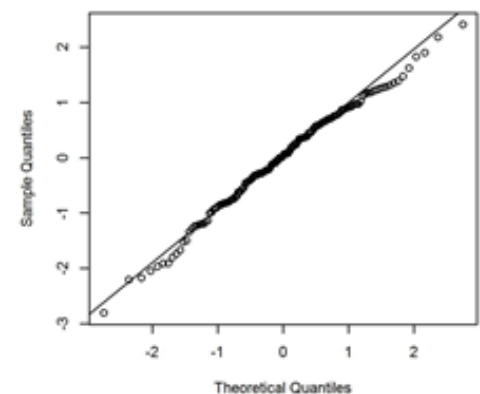

Normal Q-Q Plot

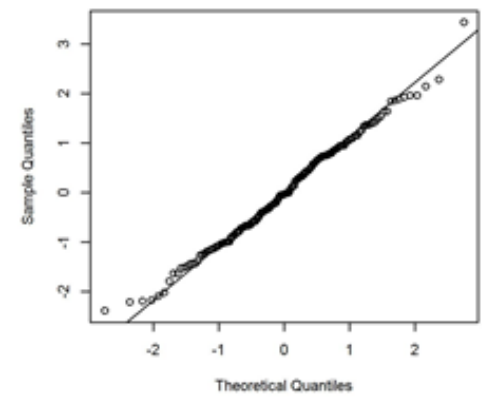

Figure A2.2. Scatterplot of the normalised residuals (left), histogram of the normalised residuals (middle), and quantile-quantile plots (right) of the Linear Mixed effects Models (LMMs) that showed significant fixed effects. a: sponge abundance $(N)$ as function of site; b: species richness $(S)$ as function of site, $N$, and interaction between these; c: Shannon's diversity $\left(H^{\prime}\right)$ as function of site, $N$, and interaction between these; d: Simpson's evenness $\left(E_{1 / \mathrm{D}}\right)$ as function of site, $N$, and interaction between these. 


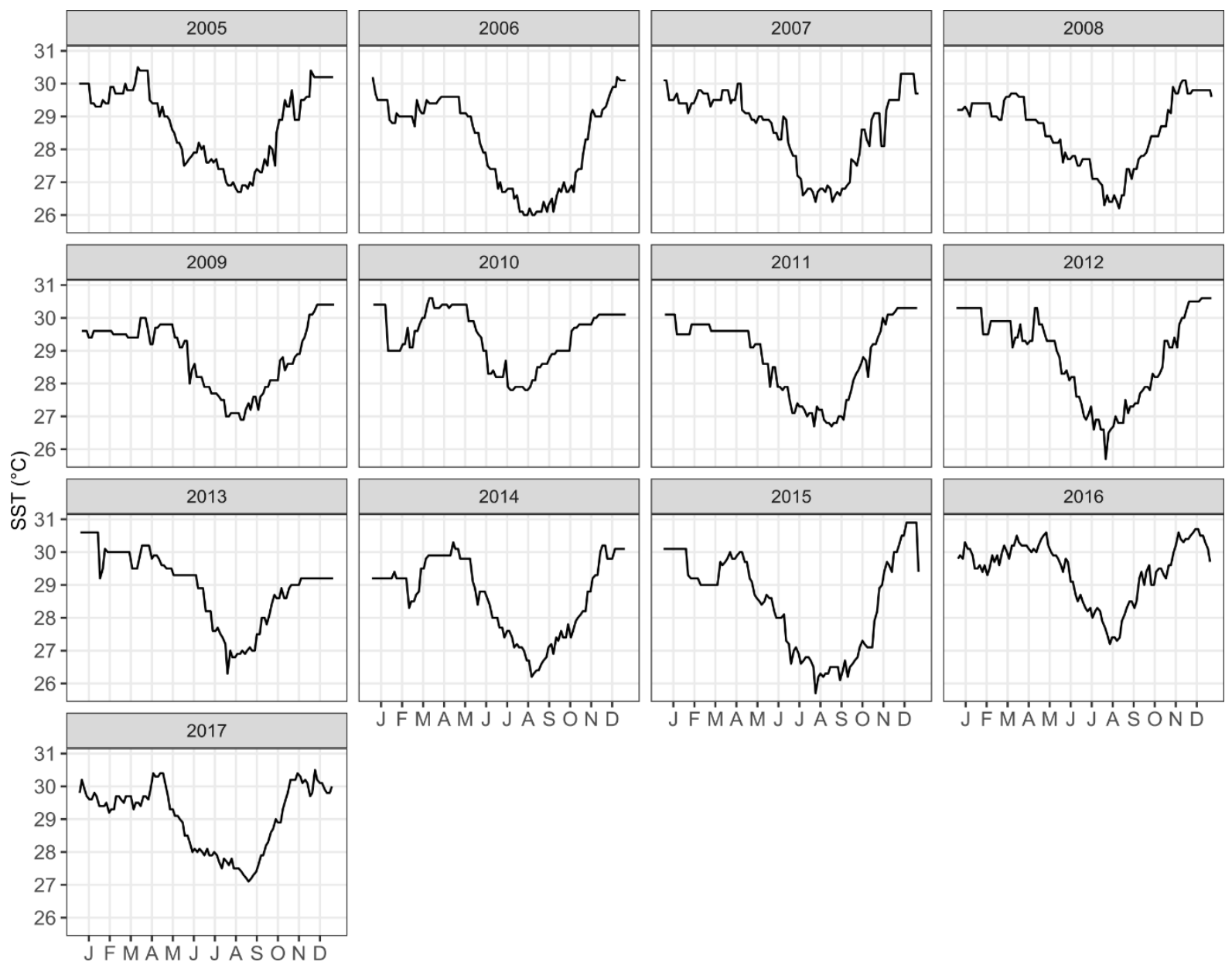

Figure A2.3. Sea surface temperature (SST) from the Wakatobi Virtual Station between 2005 and 2017. Satellite data from the National Oceanic and Atmospheric Administration's Coral Reef Watch programme $\quad$ NOAA available at https://www.ospo.noaa.gov/data/cb/TS_vs/vs_ts_Wakatobi_Indonesia.txt). 


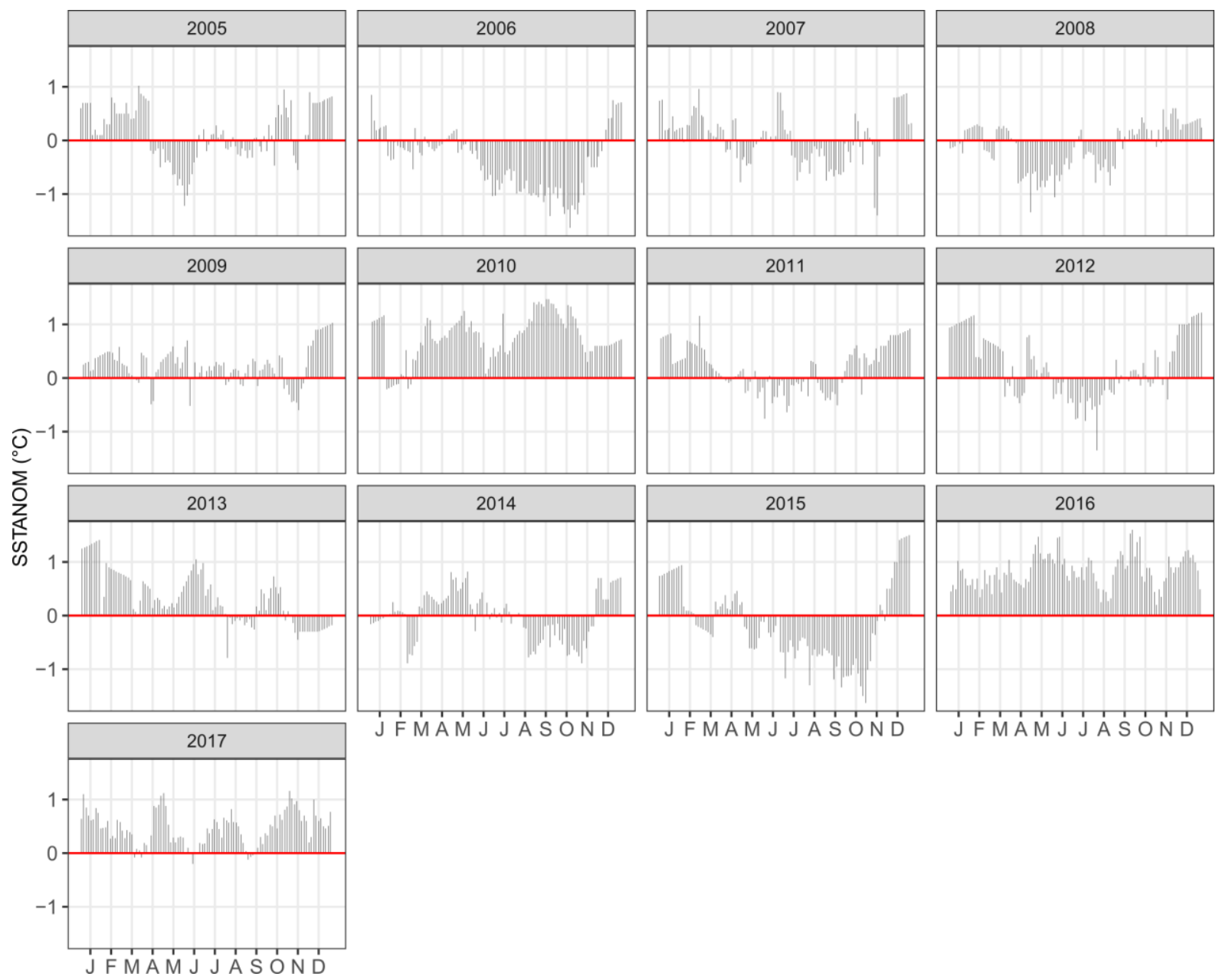

Figure A2.4. Sea surface temperature anomalies (SSTANOM) from the Wakatobi Virtual Station between 2005 and 2017. Satellite data from the National Oceanic and Atmospheric Administration's Coral Reef Watch programme (NOAA 2000, available at https://www.ospo.noaa.gov/data/cb/TS_vs/vs_ts_Wakatobi_Indonesia.txt). 


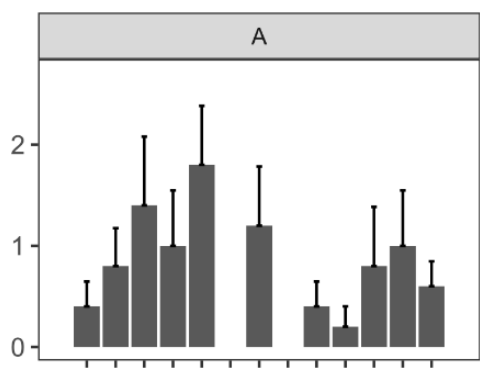

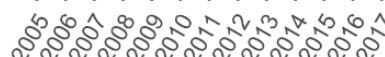
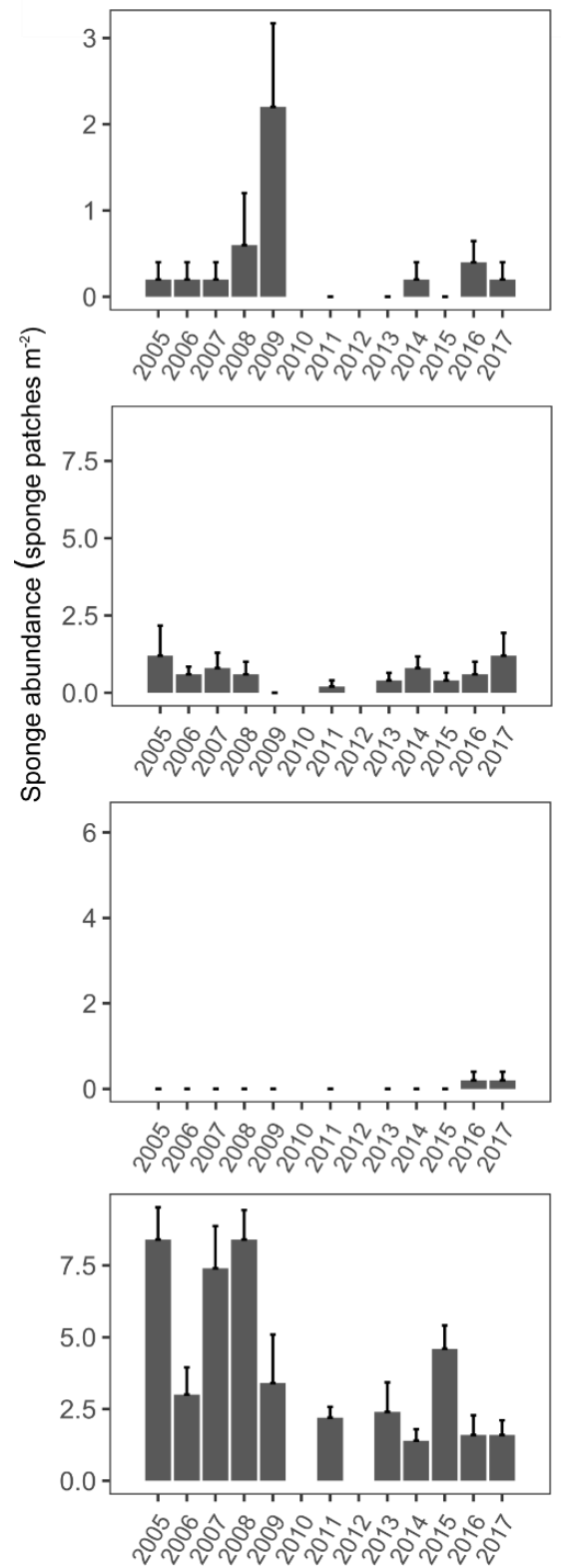
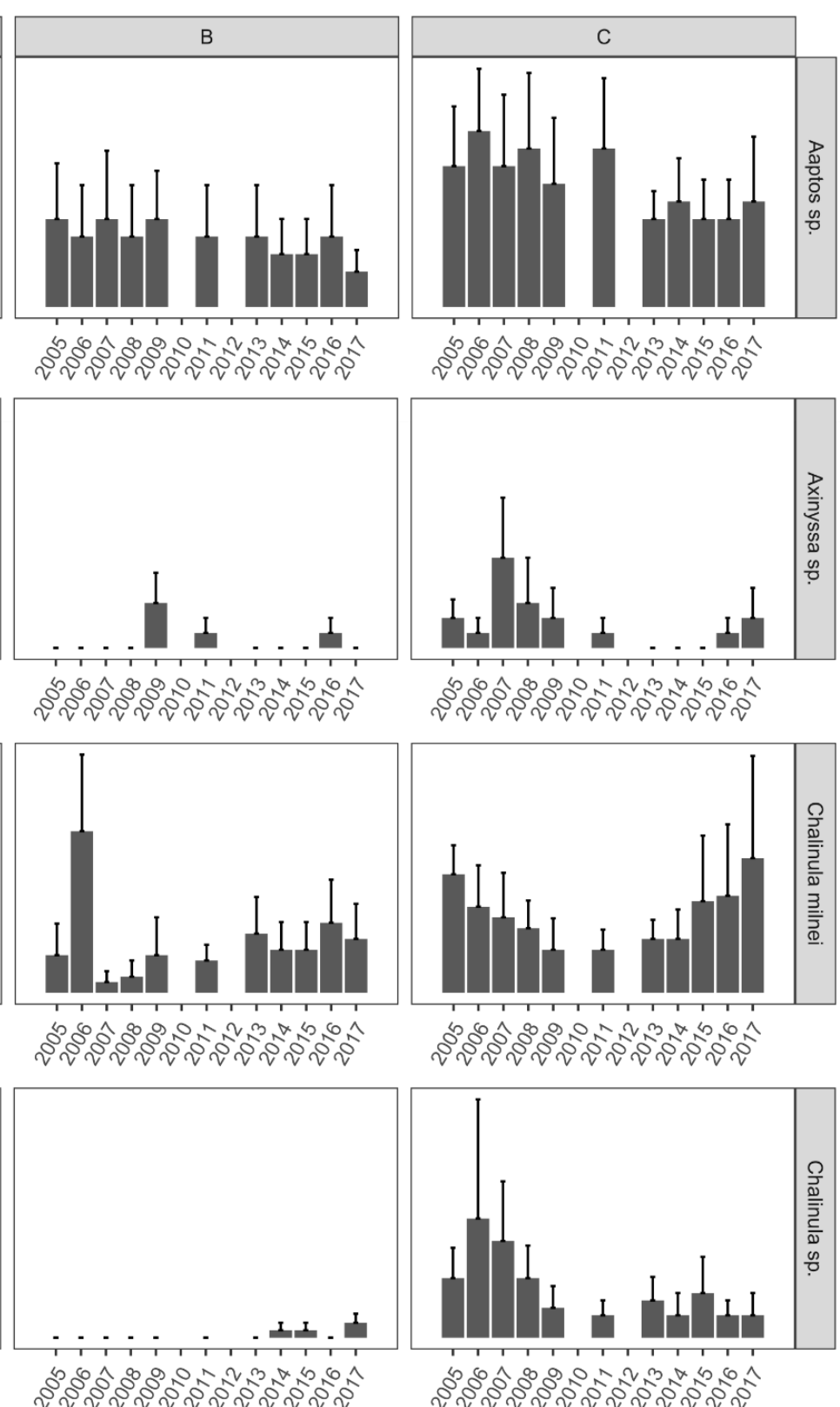

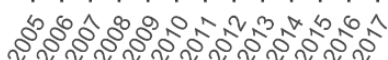
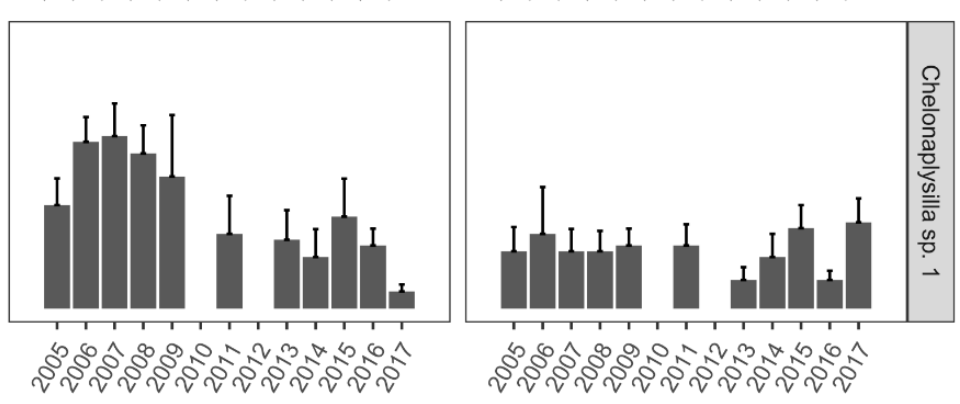

Year

Figure A2.5. Mean abundance (sponge patches $\mathrm{m}^{-2}$ ) of the most common, taxonomically identified taxa at the Hoga Island site. Of the 30 taxa that, summed, comprised $>90 \%$ of the total sponge abundance in each photoquadrat, these 25 could be identified to genus level (25 of the total 141). Note the different scales on y-axes. Error bars indicate standard error of the mean. Letters A, B and C indicate the three sites. 

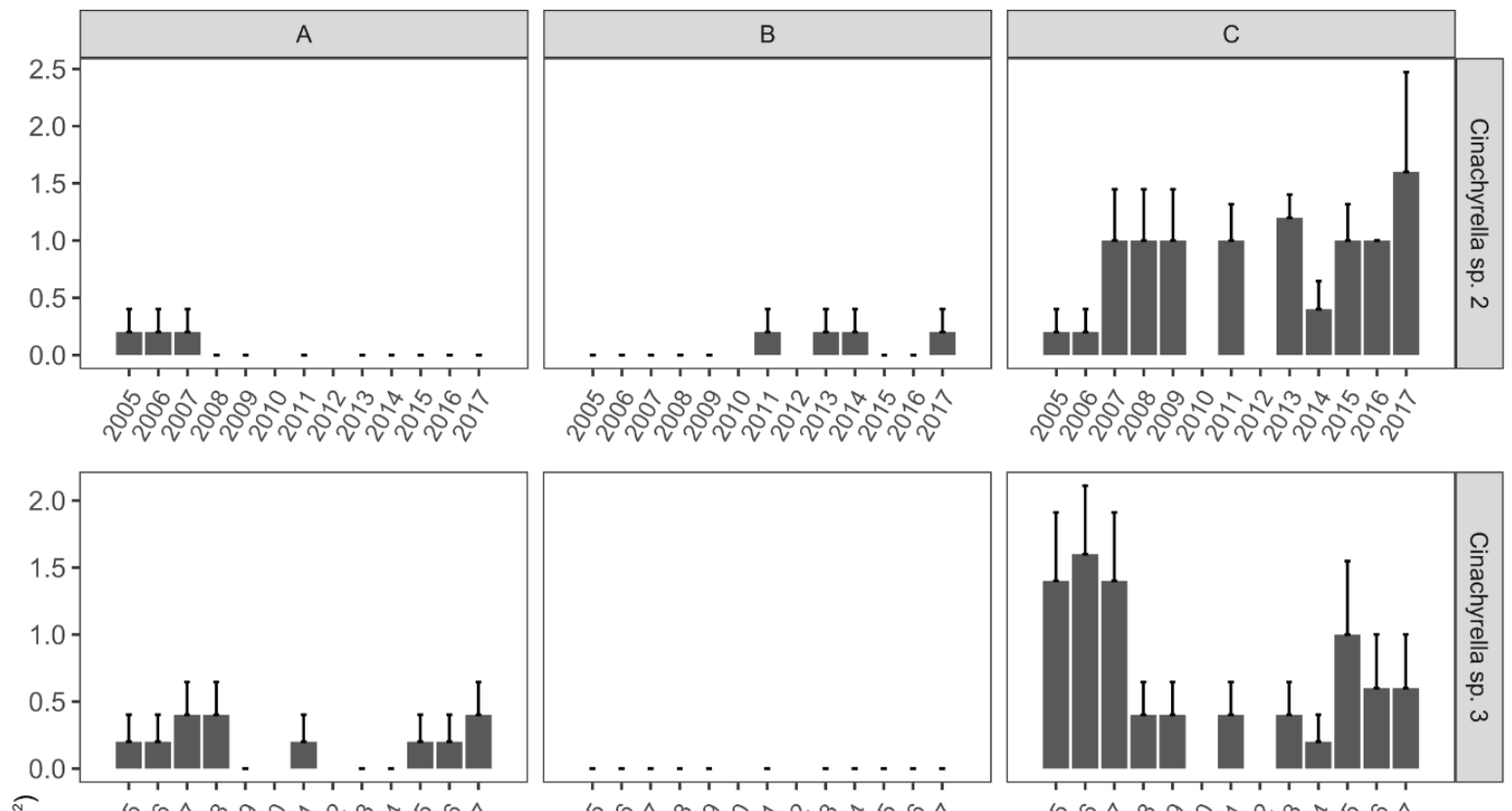

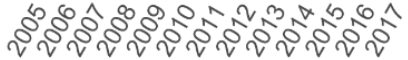

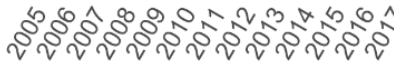
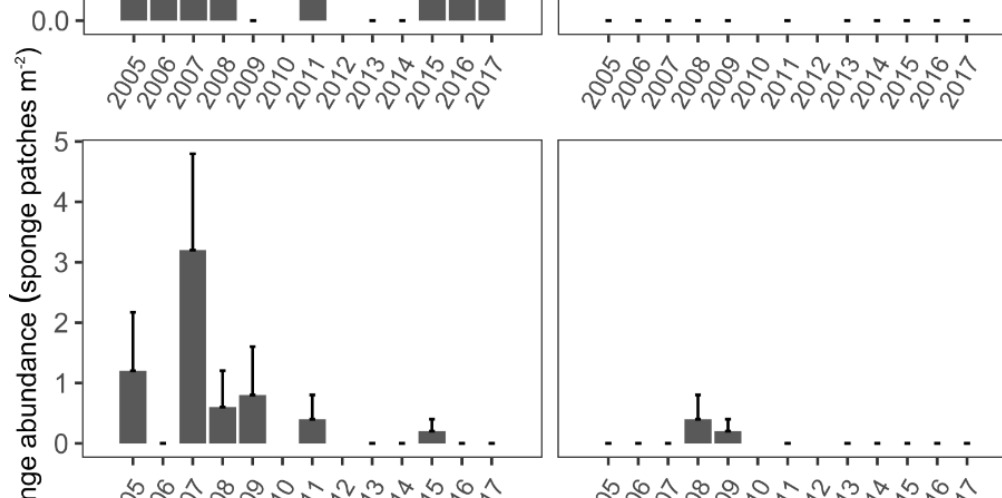

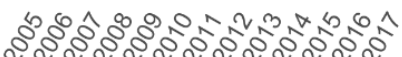

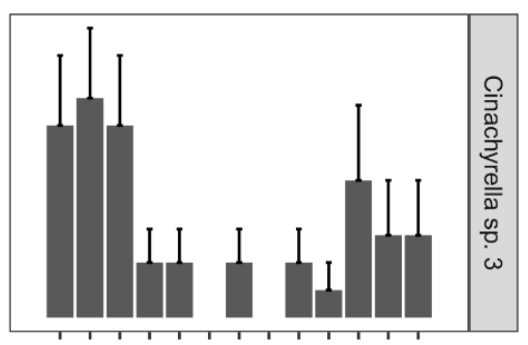

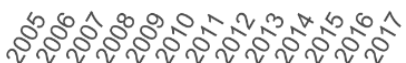
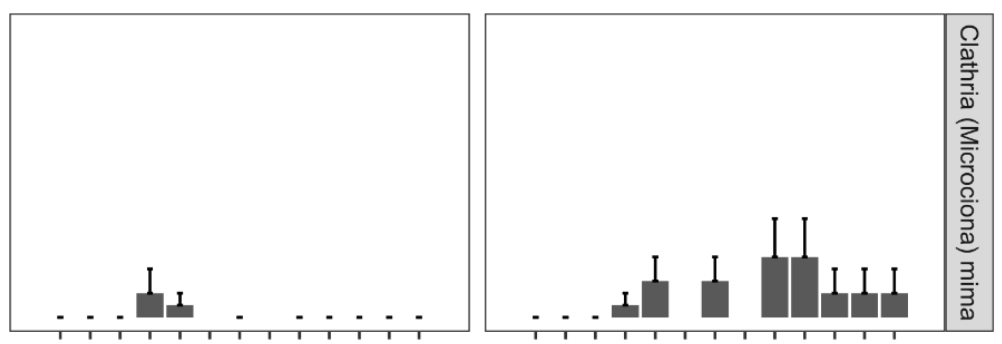

के

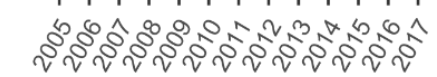

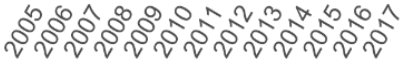
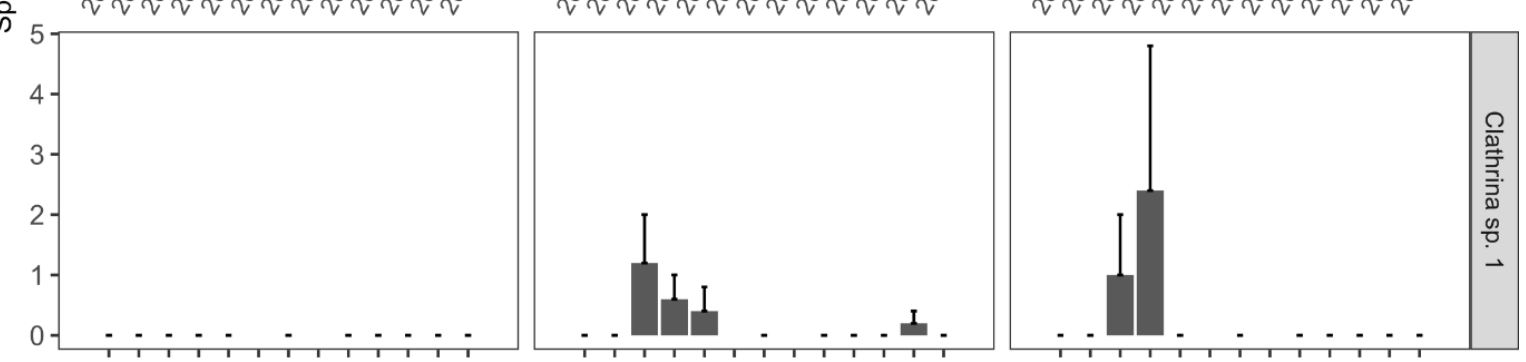

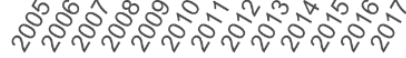

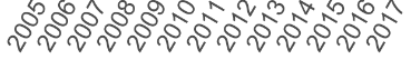
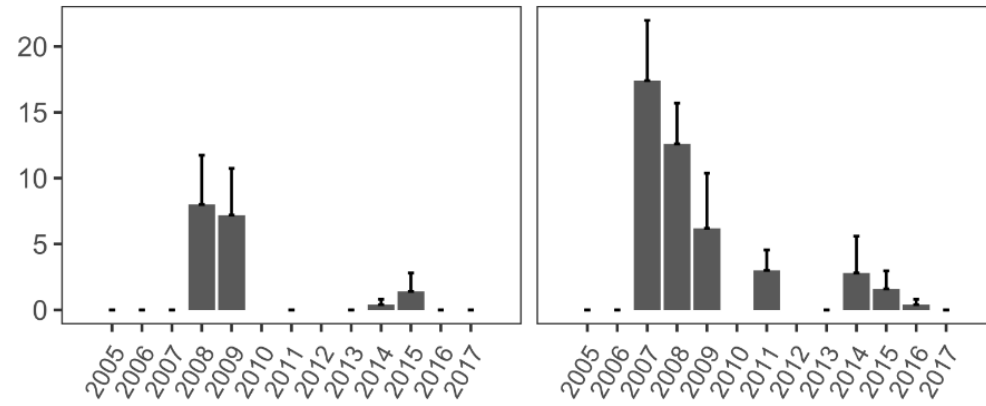

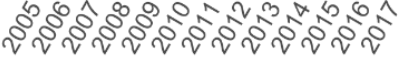

Figure A2.5: Continued.

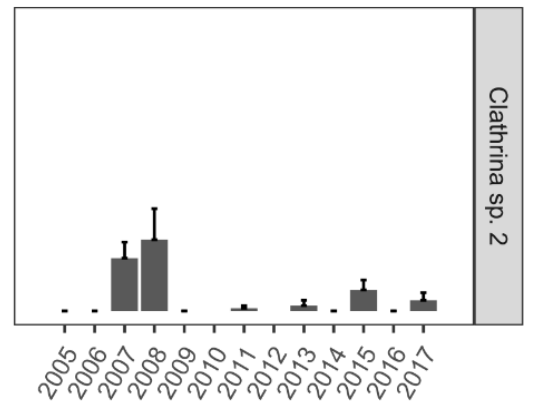

Year 


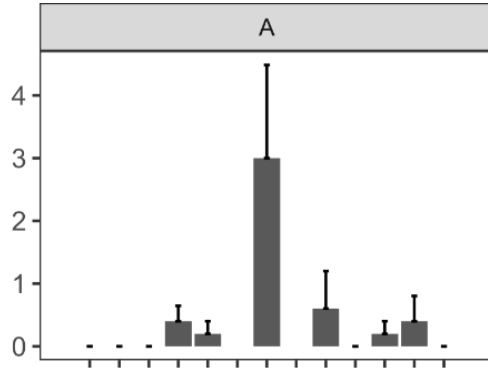

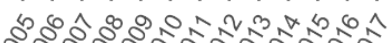

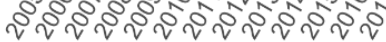

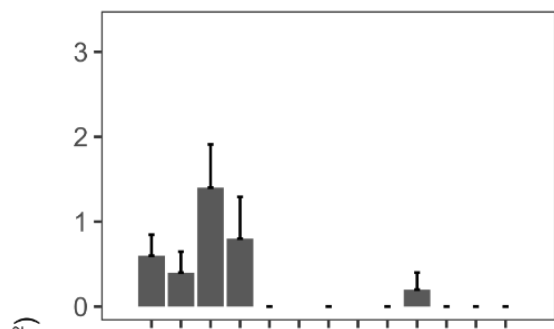$$
\text { है }
$$
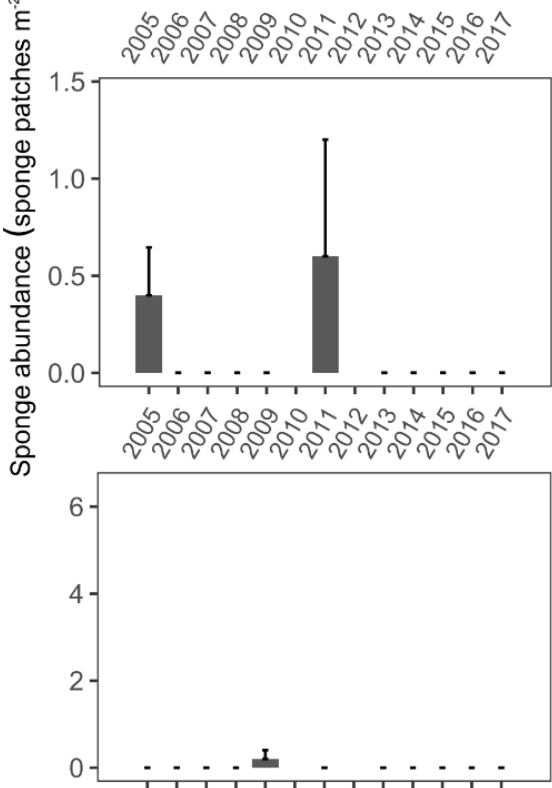

«

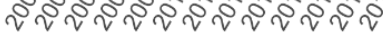

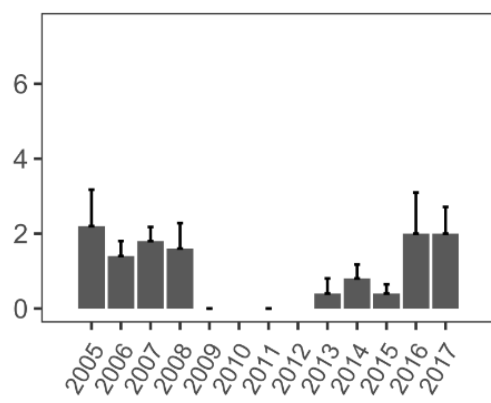

Figure A2.5: Continued.
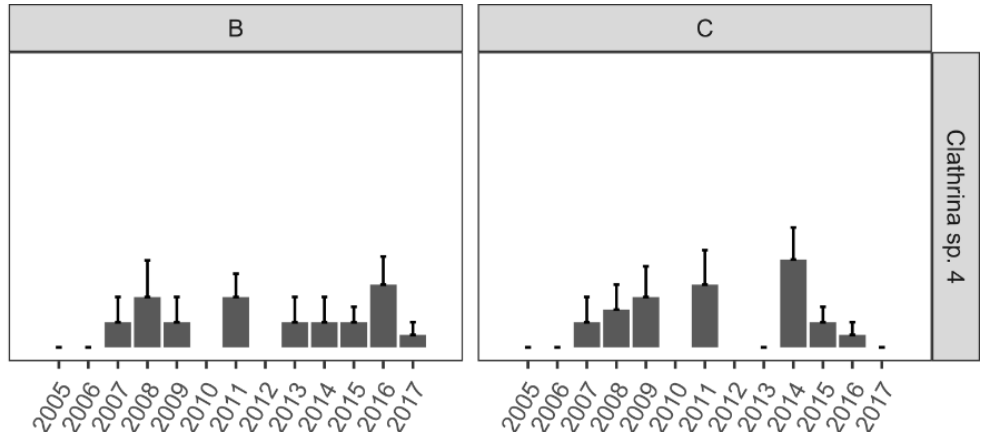

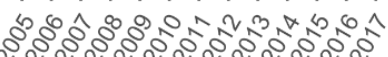

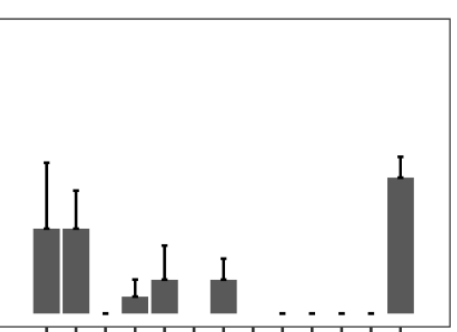

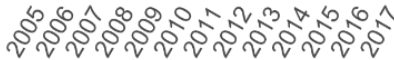
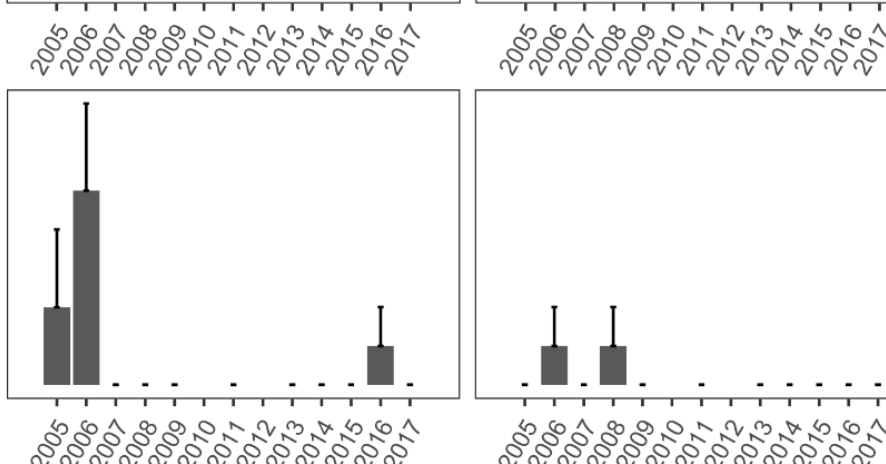

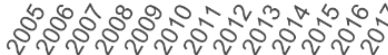

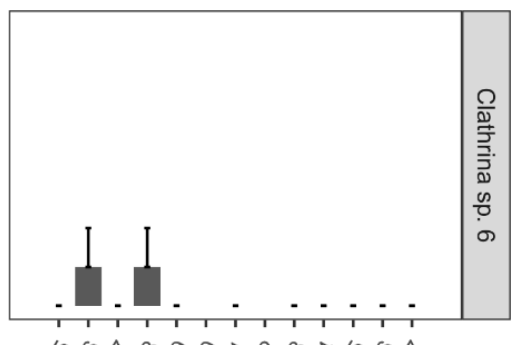

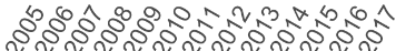

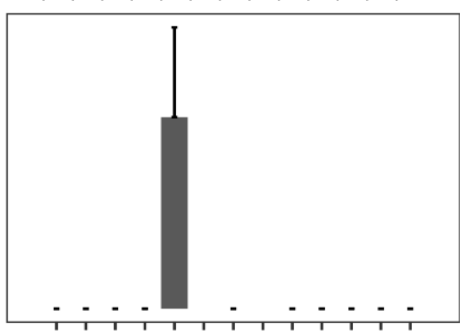

$\sin 20$

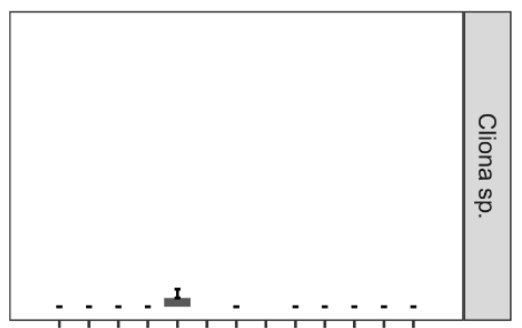

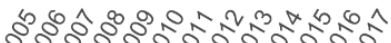

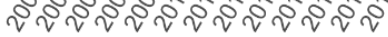

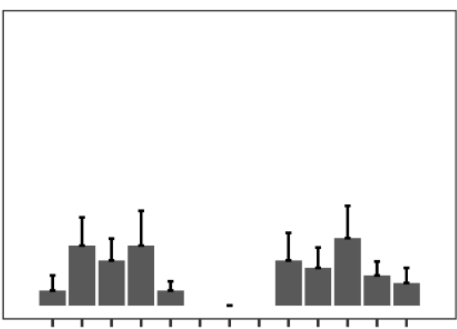

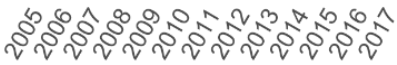

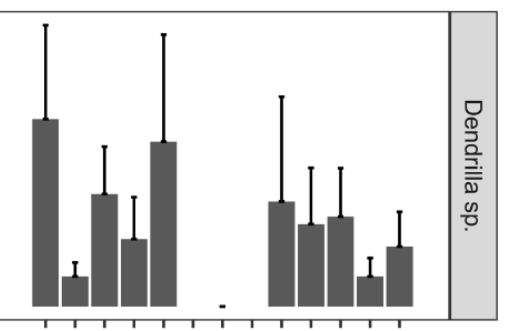

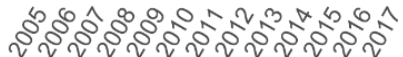



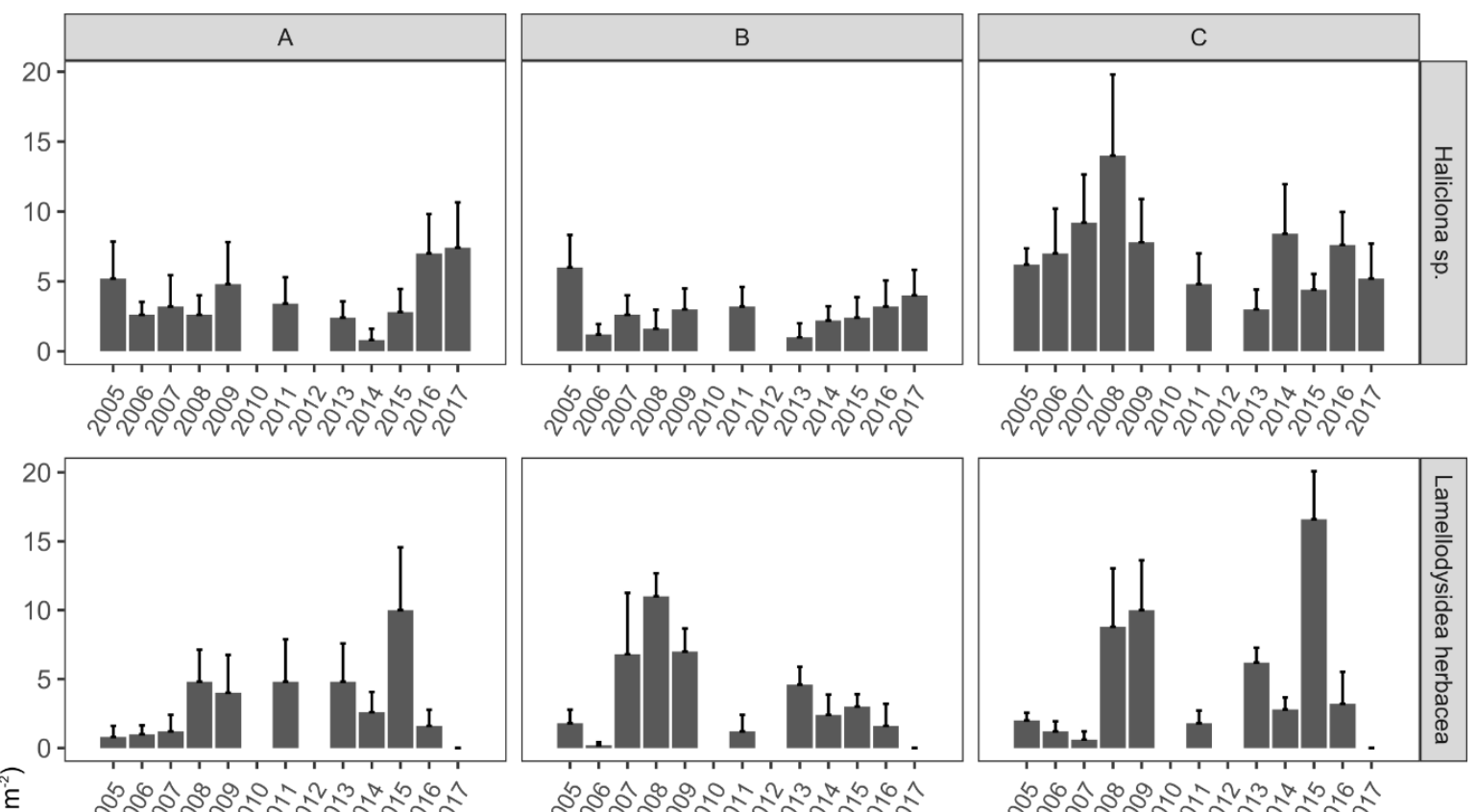

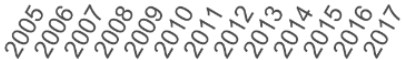

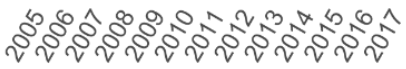
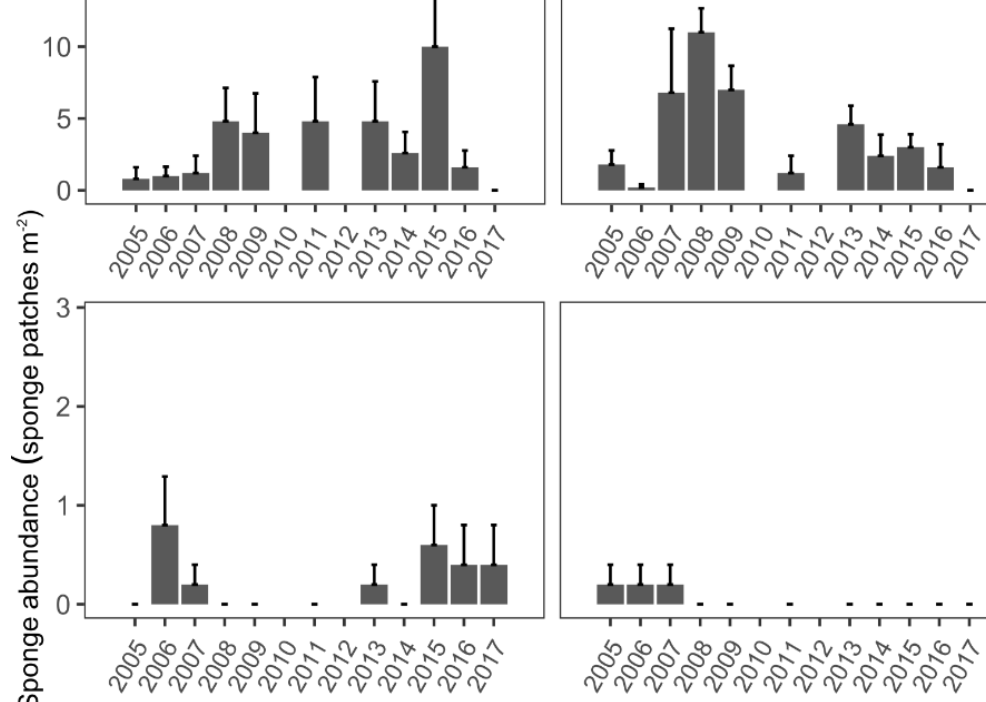

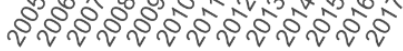

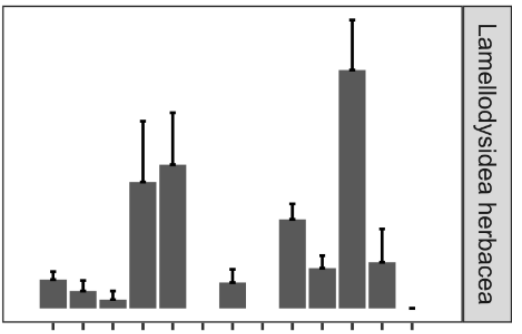

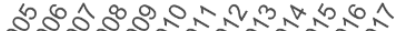

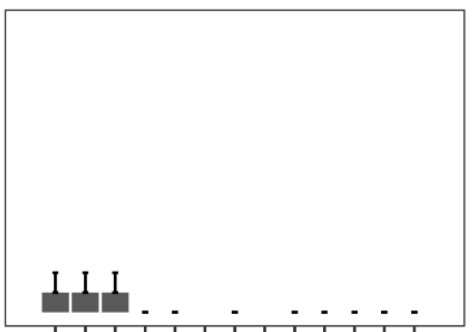

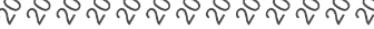

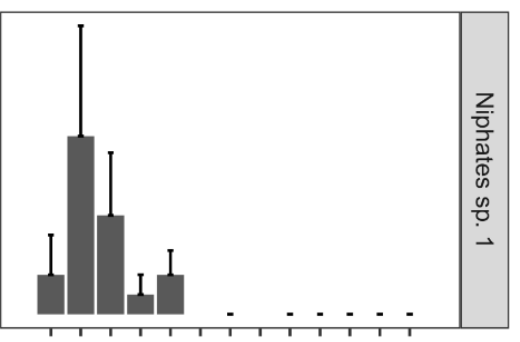

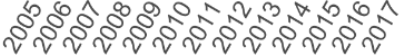

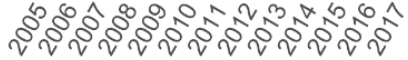
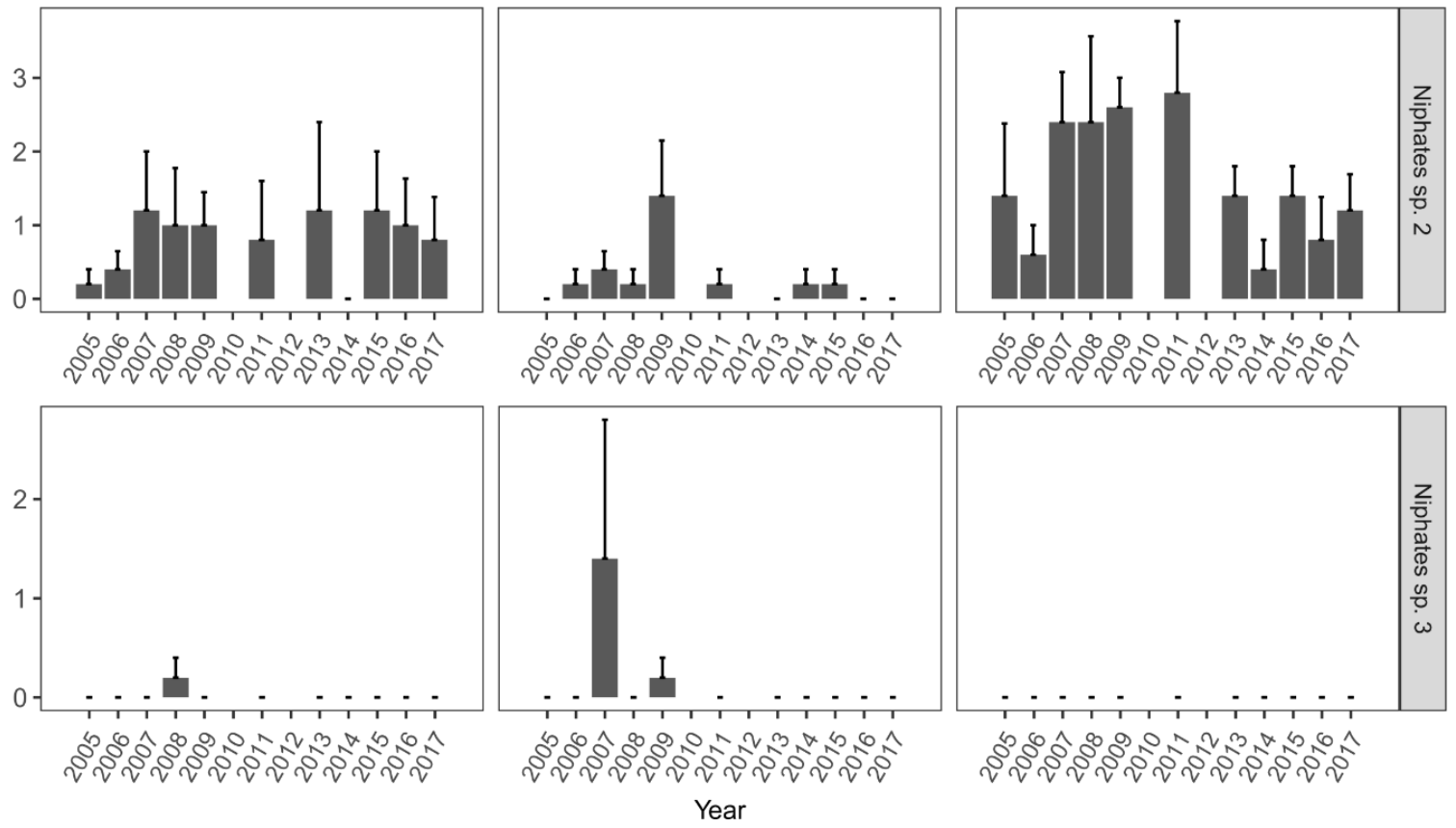

Year

Figure A2.5: Continued. 


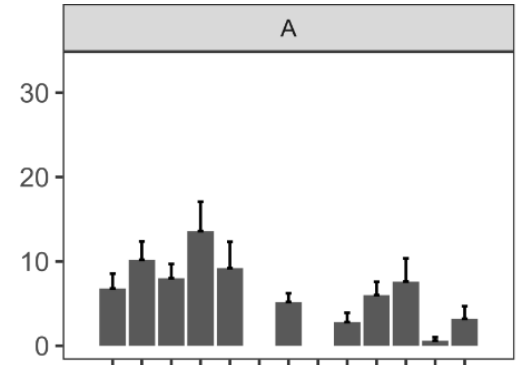

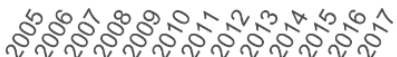

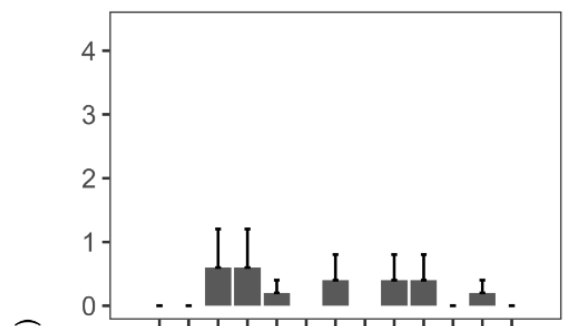

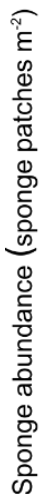
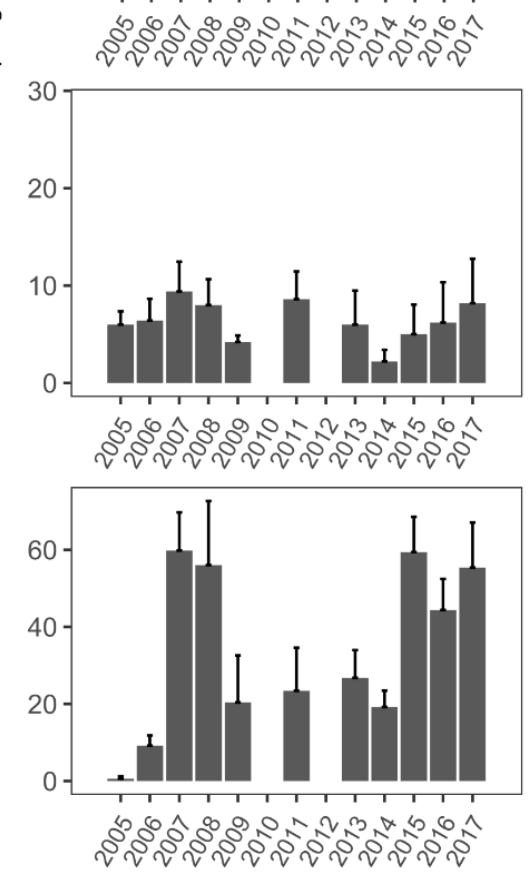

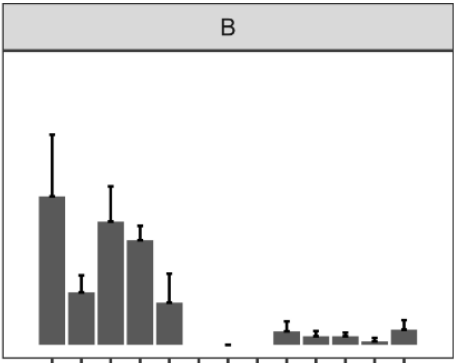

5000

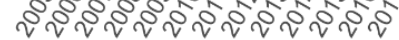
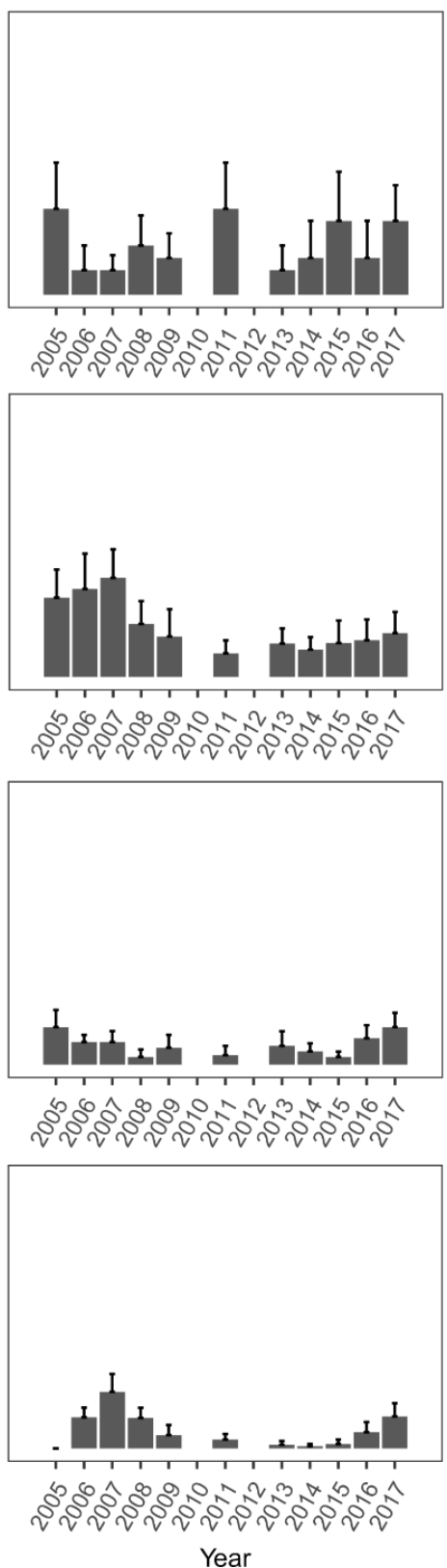

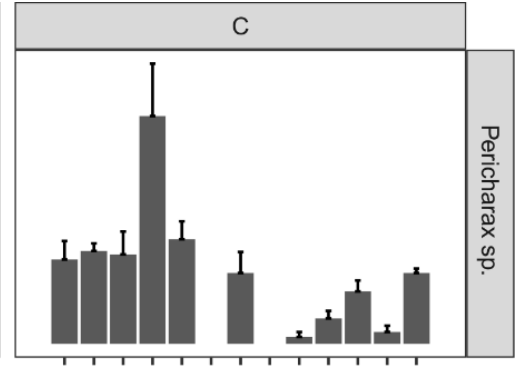

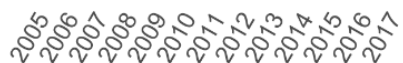

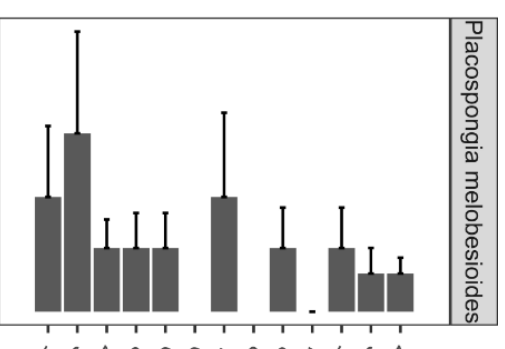

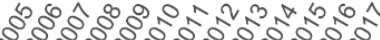

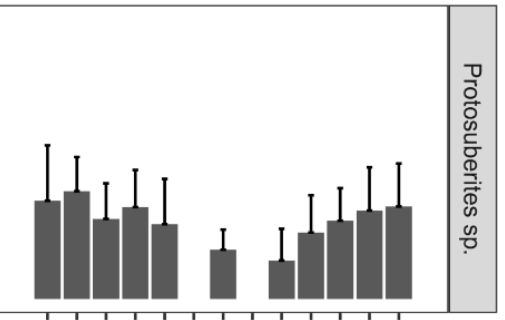

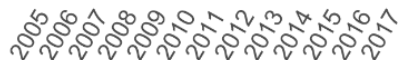
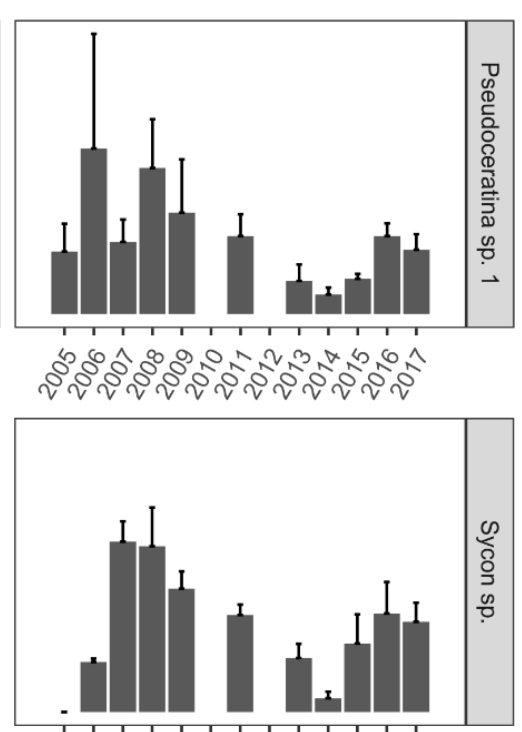

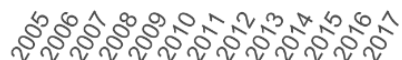

Figure A2.5: Continued. 


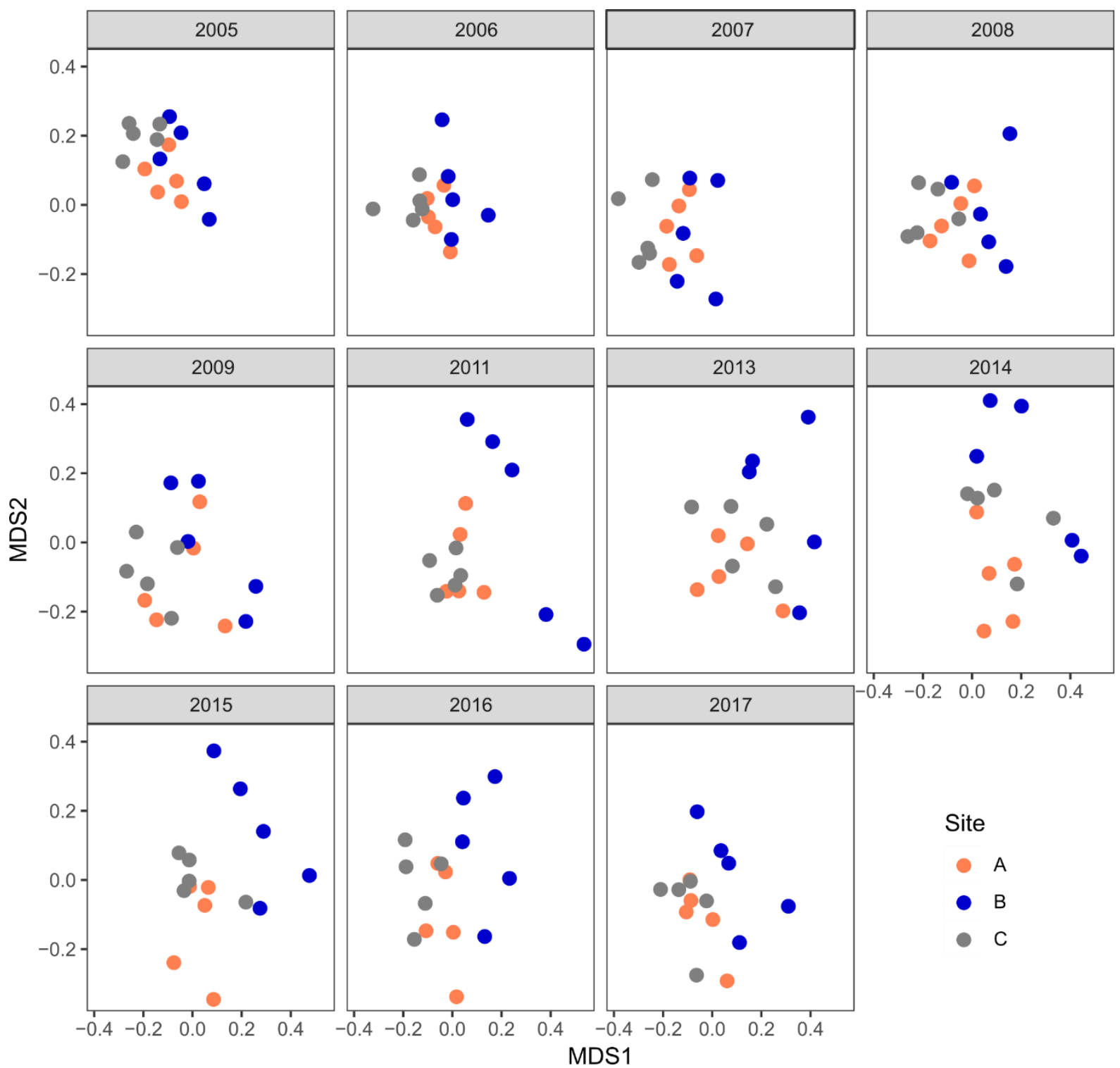

Figure A2.6. Non-metric multidimensional scaling (MDS) plot of the sponge assemblage at Hoga Island, split by survey year to aid visualisation. 


\section{Appendix 3. Decadal stability and interannual variability of benthic organisms on an Indo-Pacific coral reef}
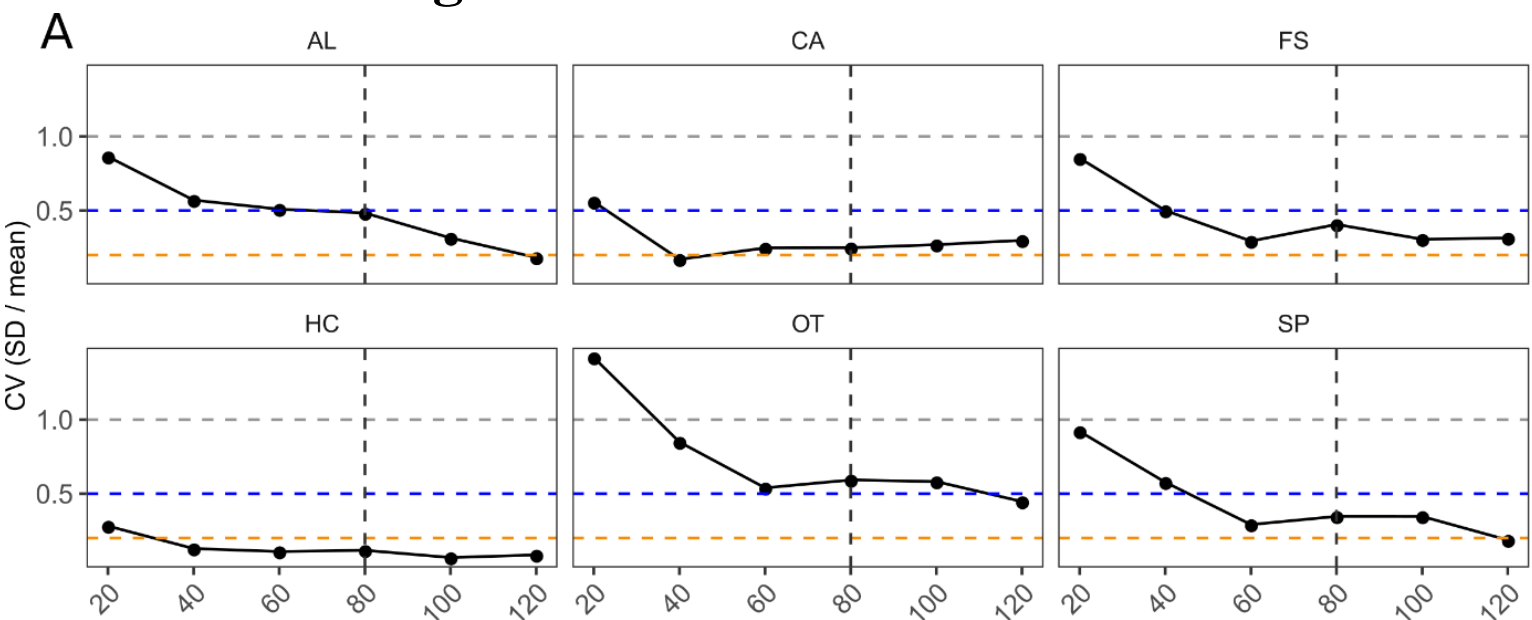

B
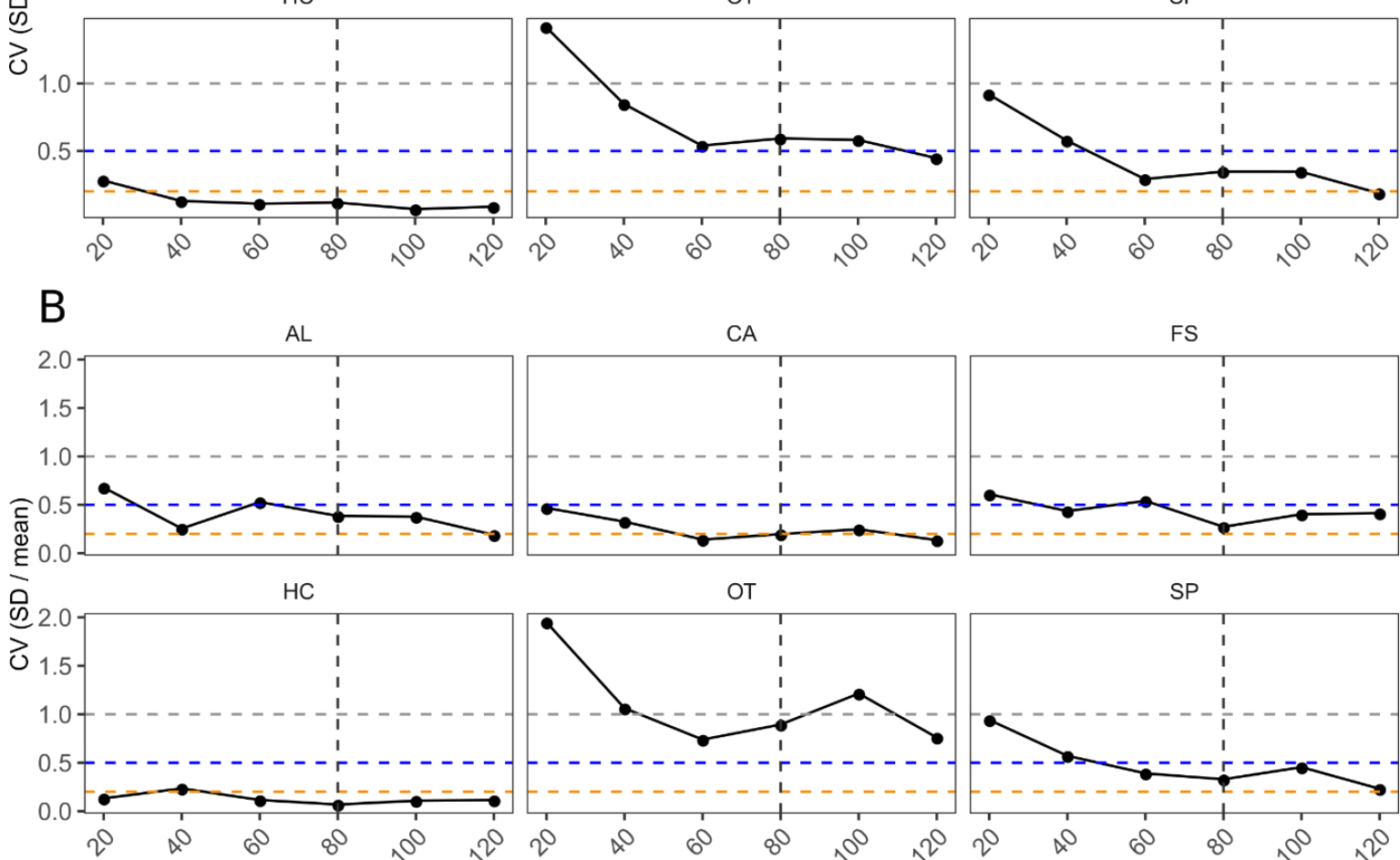

C

AL
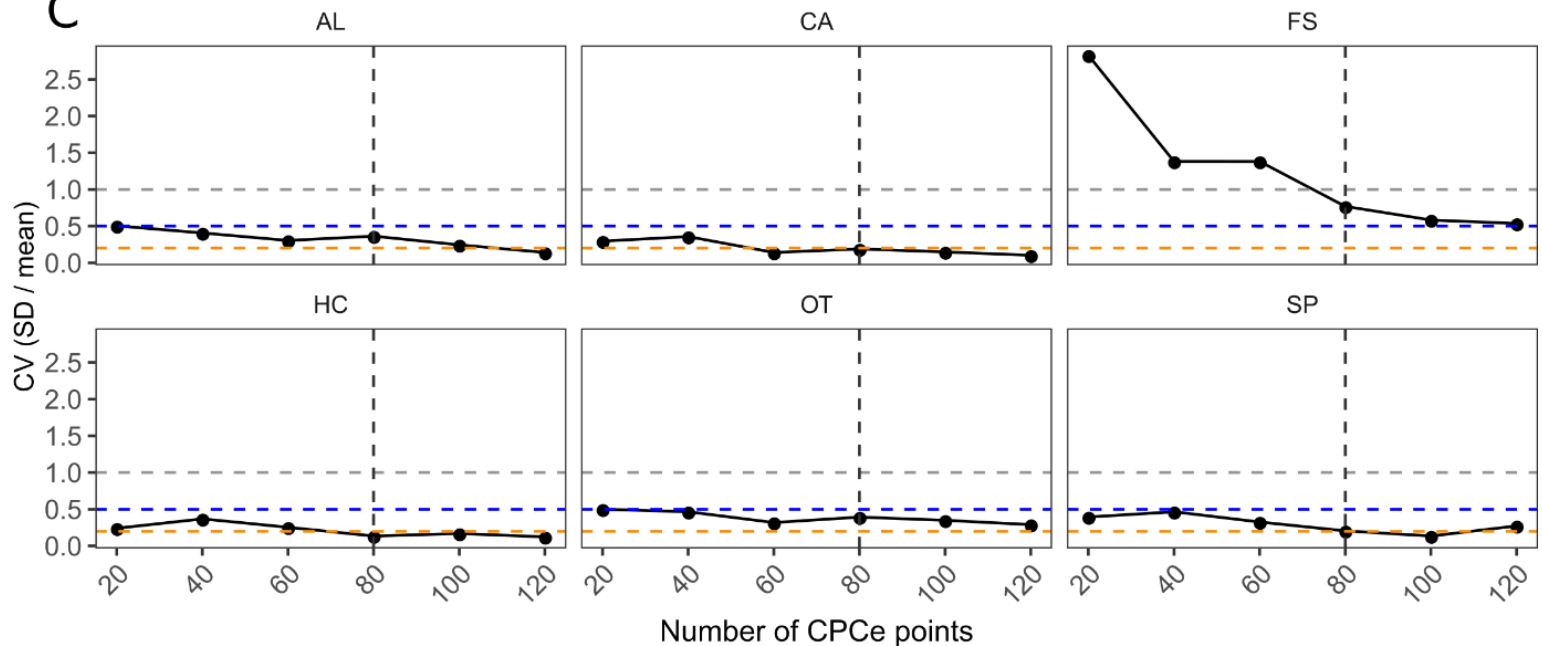

Figure A3.1. Coefficient of variation (SD / mean) obtained by averaging over values of percentage cover that had been calculated for each benthic group in 8 iterations for each amount of CPCe points (20, 40, 60, 80, 100, 120 random points per $25 \times 25 \mathrm{~cm}$ image). In each panel, 
horizontal lines indicate $\mathrm{CV}=1,0.5$, and 0.2 (grey, blue, and orange respectively); vertical dashed line indicates $80 \mathrm{CPCe}$ points / image (the chosen amount). The evaluation of CV per number of points was carried out for 3 different, randomly selected $25 \times 25$ images, indicated by letters $\mathrm{A}, \mathrm{B}$, and $\mathrm{C}$. 
a

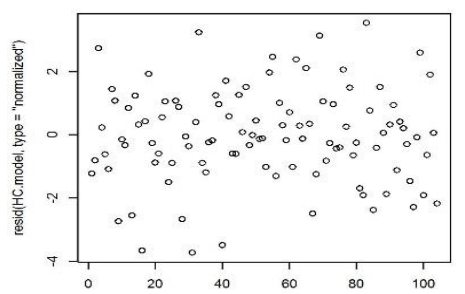

b

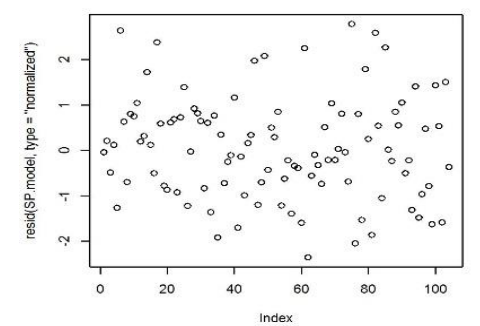

C

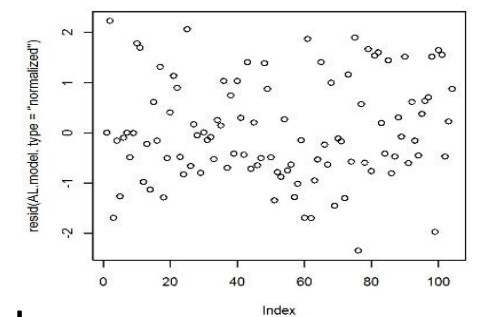

d

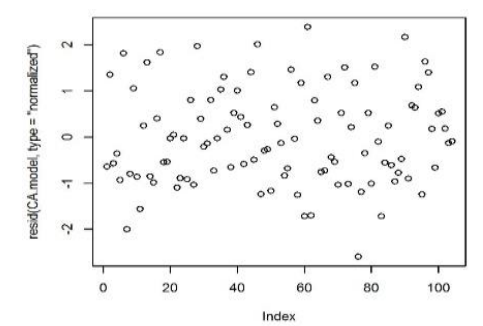

e

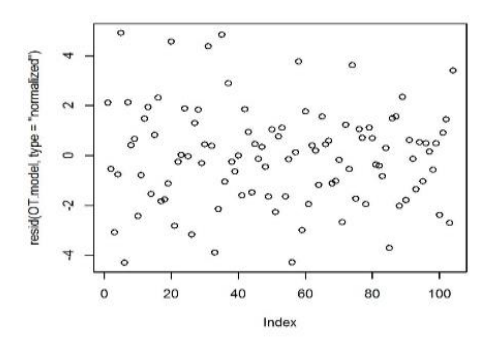

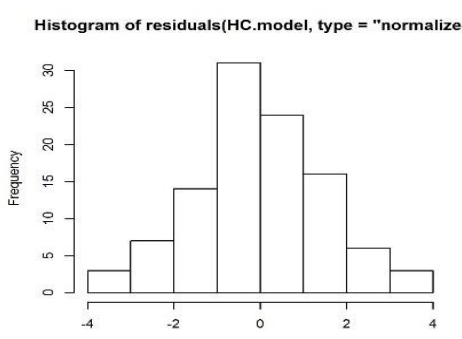

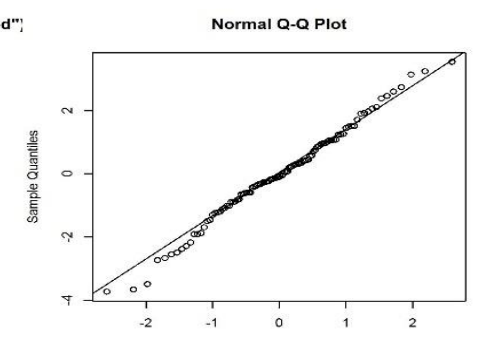

Histagram of residuals(SP.model, type = "normalized"

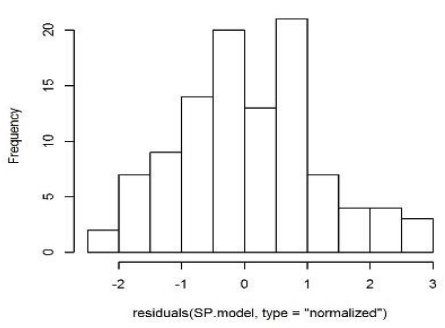

Histogram of residuals(AL.model, type $=$ "normalized")

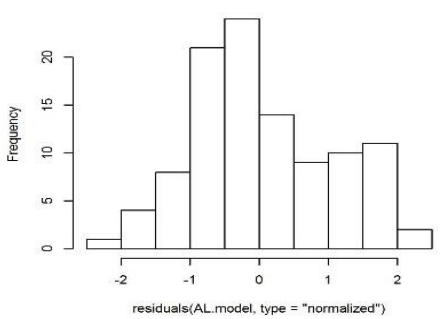

Histogram of residuals(CA.model, type $=$ "normalized"
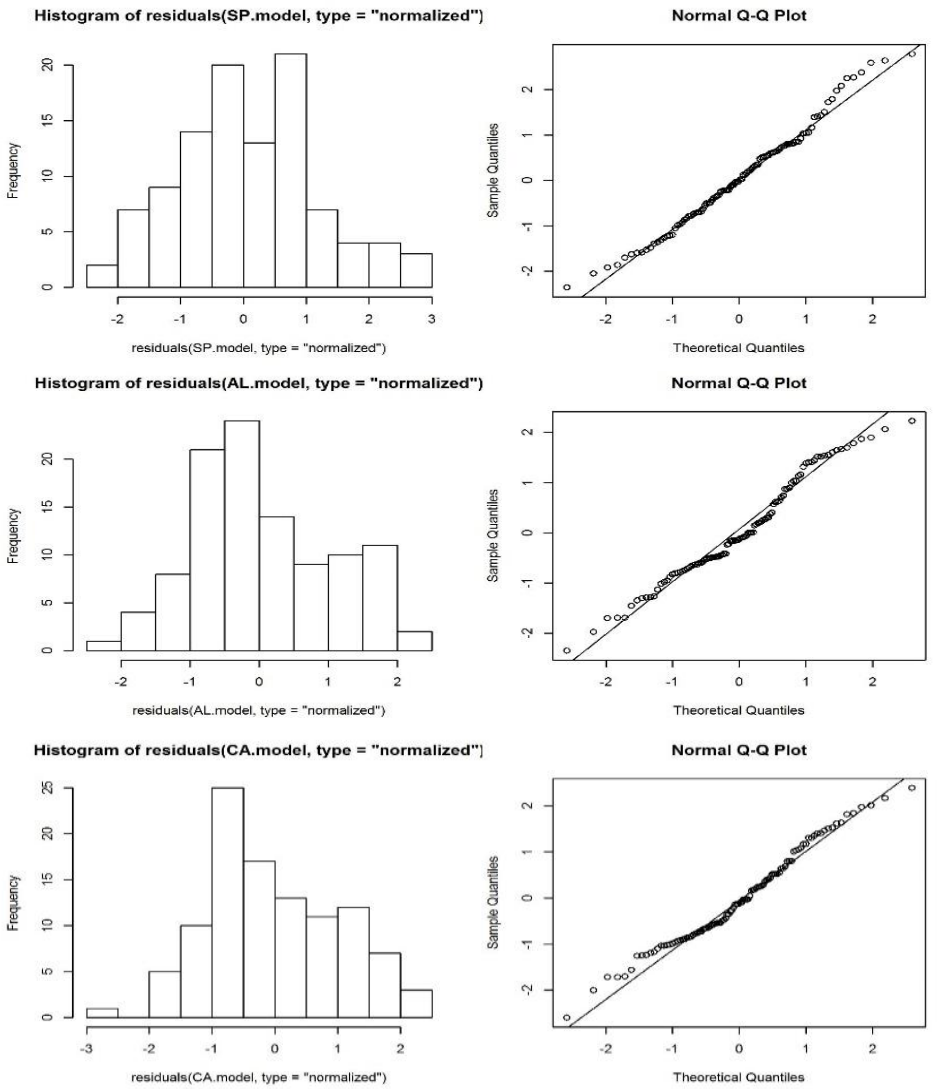

Normal Q-Q Plot

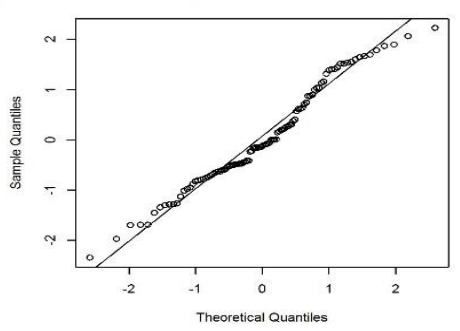

Normal Q-Q Plot

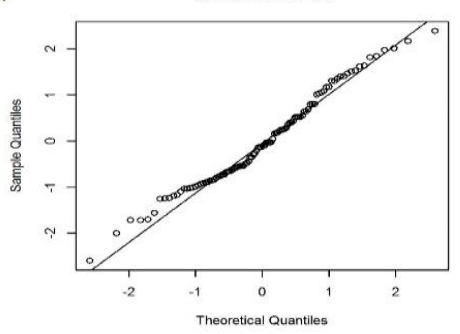

Normal Q-Q Plot
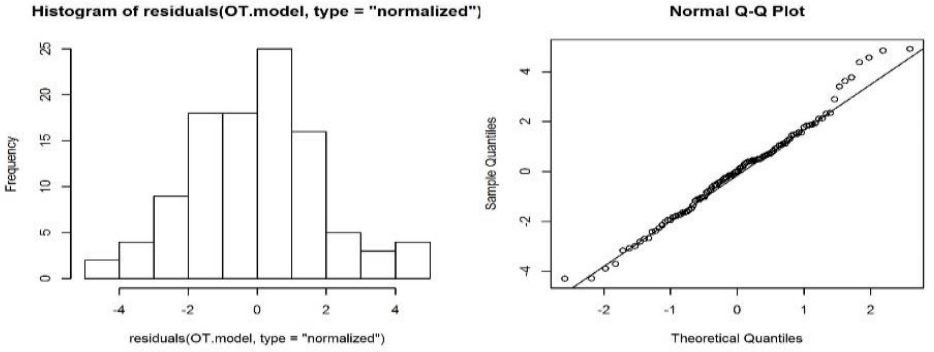

Figure A3.2. Scatterplot of the normalised residuals (left), histogram of the normalised residuals (middle), and quantile-quantile plots (right) of the Linear Mixed effects Models (LMMs) that showed significant fixed effects. a: coral \% cover as function of site; b: sponge $\%$ cover as function of site; c: algae \% cover as function of site; crustose coralline algae (CCA) $\%$ cover as function of site, year, and combination of the two; other invertebrates \% cover as function of site, year, and combination of the two. 

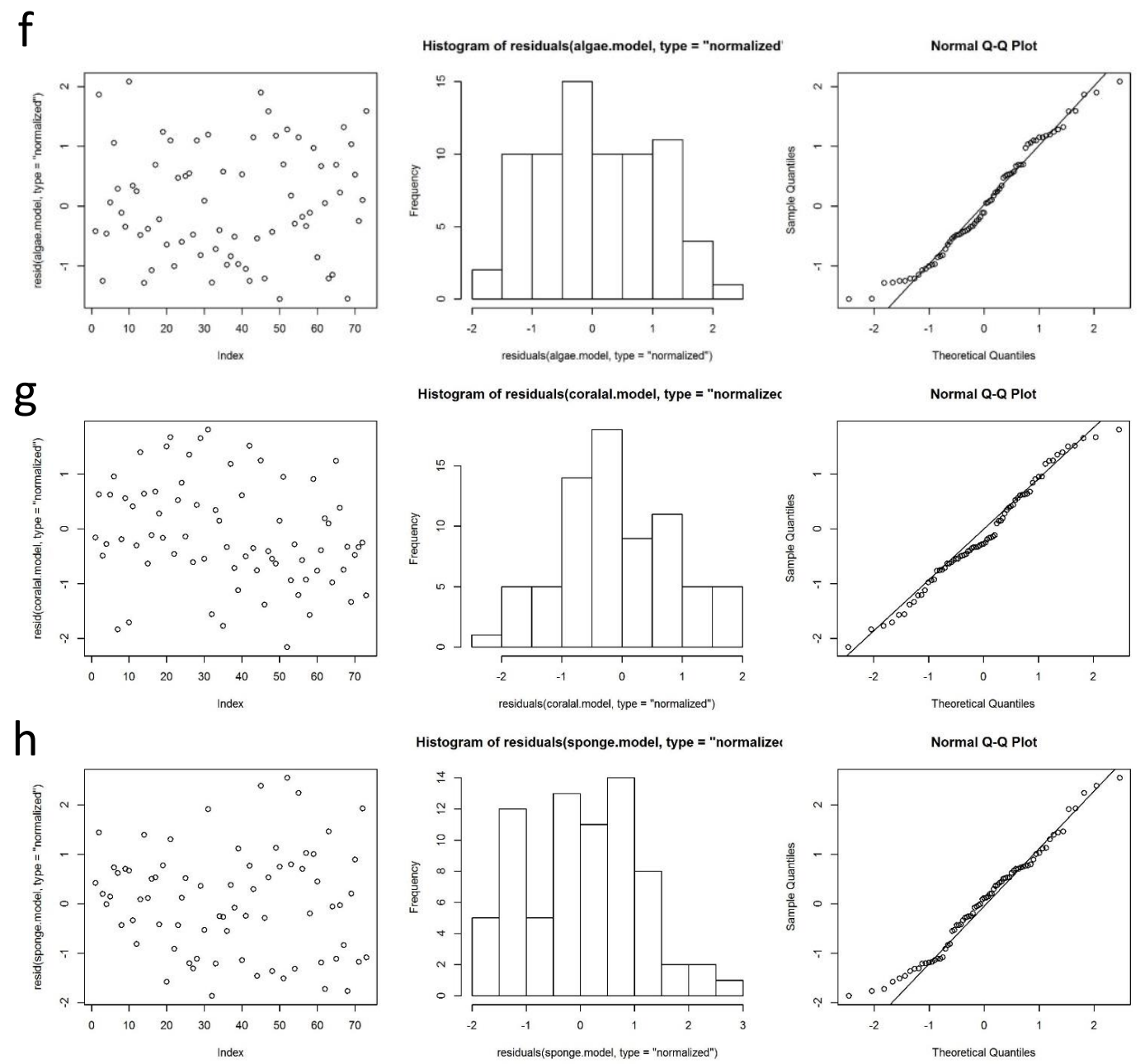

Figure A3.2 Continued. f: algae \% cover (in year $y$ ) as function of free substrate $\%$ cover (in year $y$-1) and site; g: CCA \% cover (in year $y$ ) as function of free substrate and turf \% cover (in year $y-1$ ); h: sponge $\%$ cover (in year $y$ ) as function of free substrate and turf $\%$ cover (in year $y-1)$. 


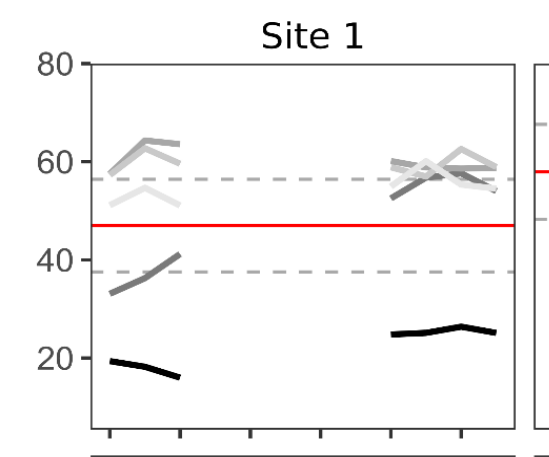

Site 2
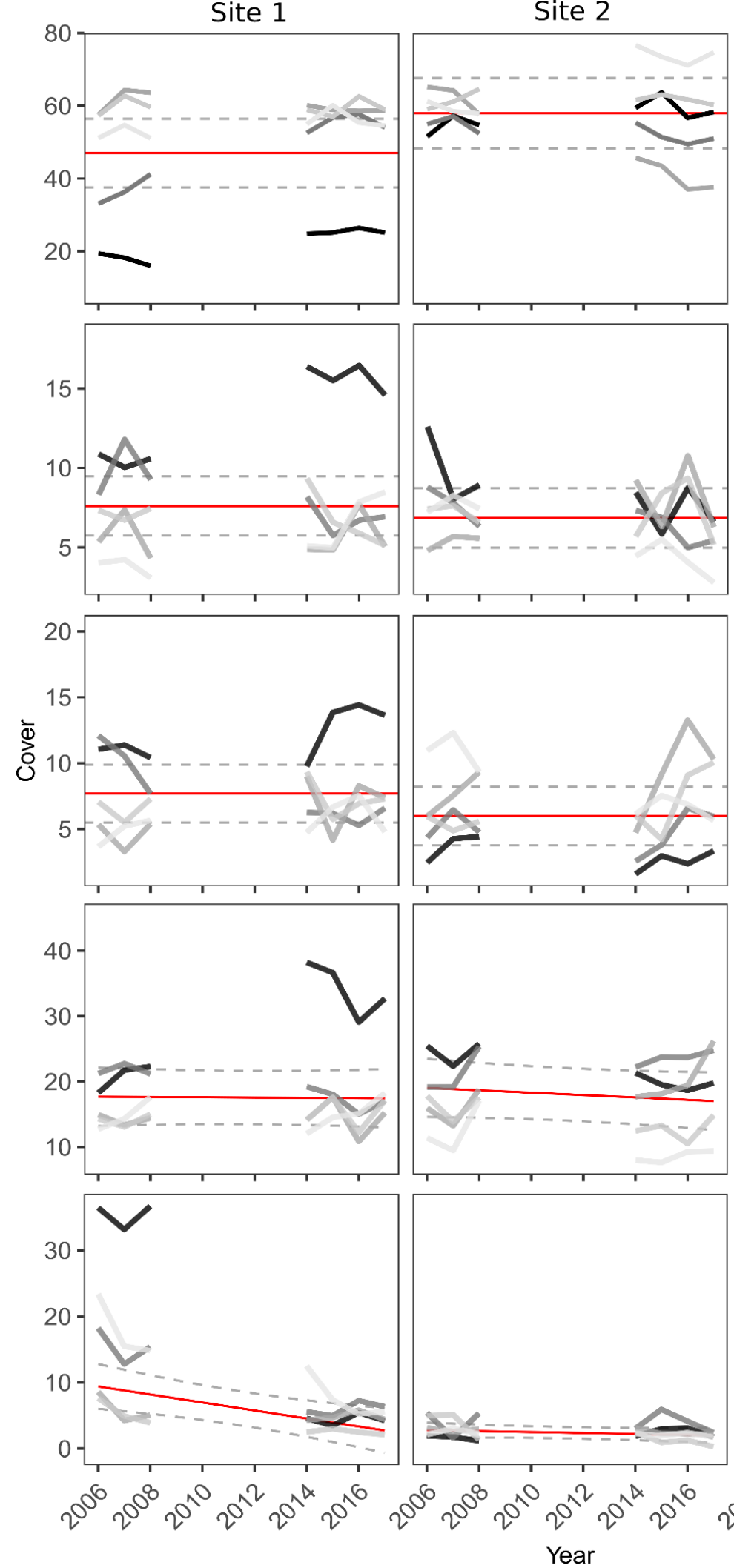

Site 3
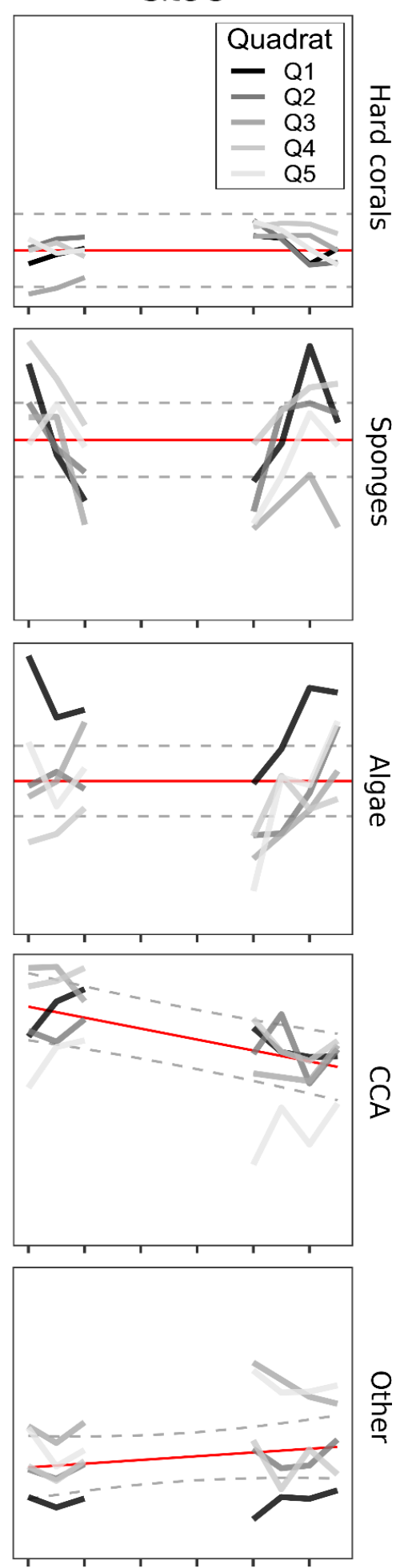

$2^{100} 2^{0^{8}} 2^{10} 2^{10} 2^{10} 2^{10}$

Figure A3.3. Temporal dynamics of the benthic groups at the three Sites on the Buoy 3 reef. Lines in different colours represent the raw data from different quadrats. Red lines represent 
the mean effects estimated by the linear mixed -effects models (LMMs), dashed lines represent $95 \%$ confidence intervals of model estimates.

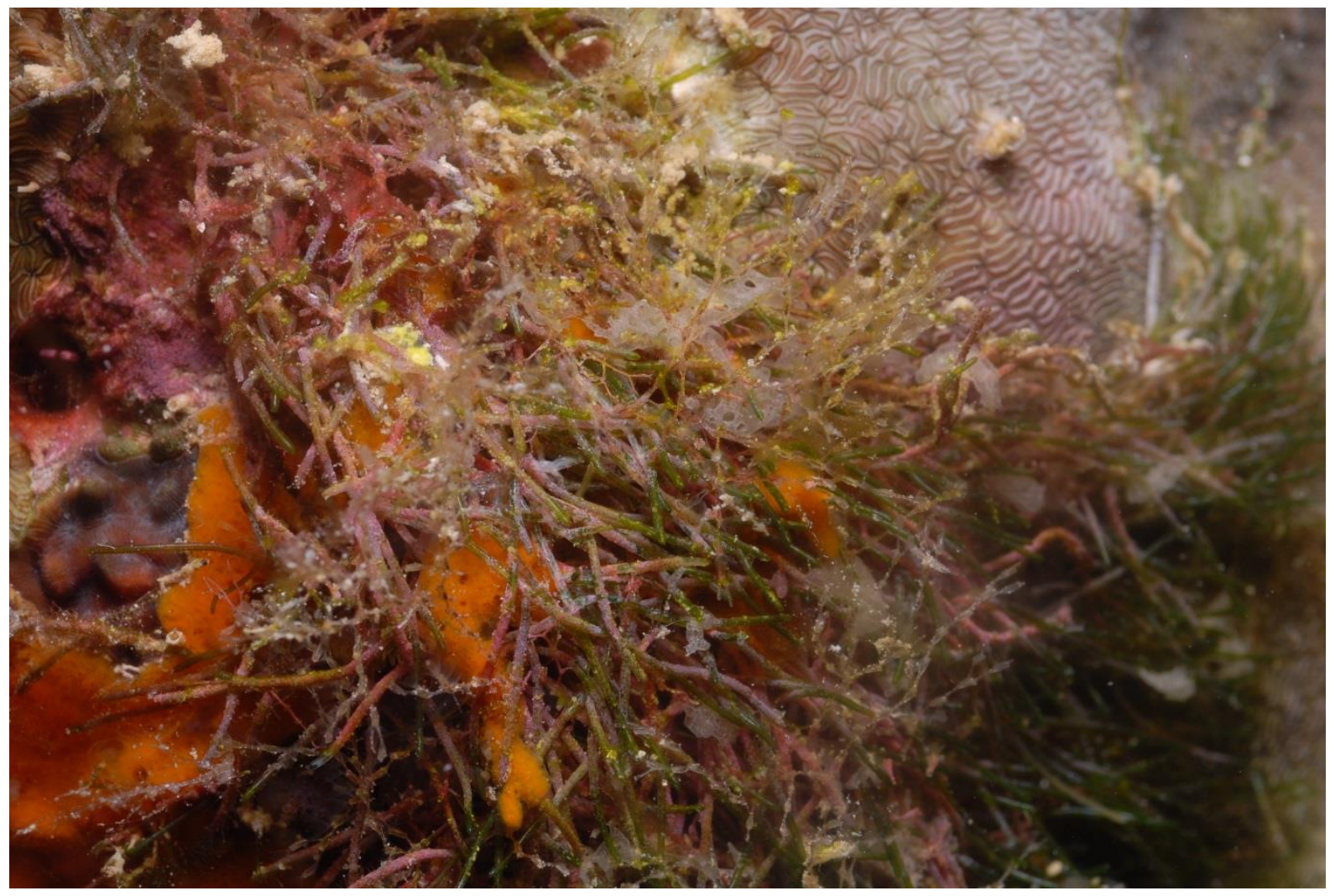

Figure A3.4. Example of association between filamentous turfing algae and a calcareous sponge (Clathrina sp., white in between algal filaments) in one of the quadrats. 